\title{
Heterogeneous Particle Deaggregation and Its Implication for Therapeutic Aerosol Performance
}

\author{
Zhen $\mathrm{Xu}$
}

A dissertation submitted to the faculty of the University of North Carolina at Chapel Hill in partial fulfillment of the requirements for the degree of Doctor of Philosophy in the School of Pharmacy.

Chapel Hill

2010

Approved by:

Dr. Anthony J. Hickey

Dr. David Leith

Dr. Jian Liu

Dr. Heidi Mansour

Dr. Russell Mumper 
(C) 2010

Zhen $\mathrm{Xu}$

ALL RIGHT RESERVED 


\begin{abstract}
Zhen $\mathrm{Xu}$

Heterogeneous Particle Deaggregation and Its Implication for Therapeutic

Aerosol Performance

(Under the direction of Dr. Anthony J. Hickey, Ph.D, D.Sc.)
\end{abstract}

The delivery of aerosol drugs using dry powder inhalers (DPIs) greatly improves the treatment of diseases such as Asthma. The major hurdles for effective treatment using DPIs relate to the formulation performance efficiency and reproducibility. The relationship between the physicochemical nature of powder formulation and the behavior of particles in the aerosolization process are still poorly understood.

In order to improve performance prediction and optimization of DPI formulation, we should have a comprehensive understanding of the physicochemical properties of the powders at rest and after being aerosolized. We hypothesize that: 1) The application of standardized entrainment tubes (SETs) for formulation performance evaluation can minimize the confounding factors caused by inhaler devices and reveal the relationship of airflow conditions and carrier-based dry powder formulation performance. 2) The models of molecular surface association described by adsorption expressions can be adapted to fit shear displacement observations. 3) The carrier surface treatment can reduce the surface heterogeneity and improve the performance efficiency of dry powder aerosol formulation. 
A set of SETs was used to allow focus on the relationship between formulation and their aerosolization performance. A novel interpretation using powder aerosol deaggregation equation (PADE) was developed based on comprehensive studies of carrier-based formulation performance.

The PADE led to a fundamental understanding that the forces acting at the particle interface are analogous to those at the molecular level, and the models of molecular surface association described by the adsorption theory are analogous to shear displacement. The analogy from surface adsorption/desorption led to a completely novel way of looking at particle interactions. Several related surface phenomena were compared from their physical meanings to the fundamental theories.

The PADE was further challenged by coating of lactose carrier particles with fatty acids. The coating was found to effectively improve the aerosolization performance, presumably by means of reducing surface heterogeneity and masking high surface energy density regions. Using SETs in which particle separation occurs in response to shear stress, we have developed a basic understanding of aerosolization performance described by PADE. This observation may serve as a link between macroscopic statistical thermodynamics and microscopic individual particle deaggregation. 


\section{ACKNOWLEDGMENTS}

I cannot overstate my deep gratitude to my major advisor, Dr. Anthony Hickey for his great guidance and full support. He led me into the realm of aerosol drug delivery and inhaled particle science. I felt being blessed in my entire Ph.D. pursuit, owing to Dr. Hickey's invaluable guidance, encouragement, and patience. I esteem it a great honor to work for such a respectable and knowledgeable professor.

I would like to cordially thank each of my dissertation committee members, Dr. David Leith, Dr. Jian Liu, Dr. Heidi Mansour, and Dr. Russell Mumper for their precious suggestions and time spent. Dr. Mansour constantly helps me from various aspects of my research progress including data interpretation, presentation skills, and down-to-earth attitude toward research. Working with Dr. Mansour has been a great and cherished experience in my life. Dr. Leith is a great instructor and an aerosol guru. The Aerosol Technology class taught by him helped me understand and interpret many situations in my research studies. Many thanks to Dr. Liu and Dr. Mumper, for their great guidance in the committee meeting and defense.

I'm grateful for Dr. Margaret Louey from GSK for expert discussion of using standardized entrainment tubes (SETs), Wayne Jones and Mike Smurthwaite from Westech for construction of additional SETs, Dr. Gary Pollack and Dr. Jeannie Padowski for their expert assistance on gas chromatography quantification, Dr. Ambrose Wallace for use on the scanning electron microscopy, Dr. Peter White for instruction on the X-ray powder 
diffraction analysis, Dr. Michael Chua for providing access to the Michael Hooker Medical Microscopy Facility, Dr. Michael Falvo for expert discussions on atomic force microscopy, and Dr. Debbie Kraus from Pfizer for lactose batch selection based on statistical analysis.

I would like to acknowledge all current and former members of the Dispersed Systems Laboratory, especially: Lucila Garcia-Contreras, Pavan Muttil, John Fallon, Dongmei Lu, Robert Garmise, Dan Cooney, Chenchen Wang, Sheena Wang for their tremendous help and friendship. I would also like to thank my colleagues in the School of Pharmacy: Xin Ming, Rong Zhao, Bo He, Wujian Ju, and many others who helped me in my research and presentation.

Finally, I would like to thank my parents and my wife for their love and support. 


\section{TABLE OF CONTENTS}

\section{Pages}

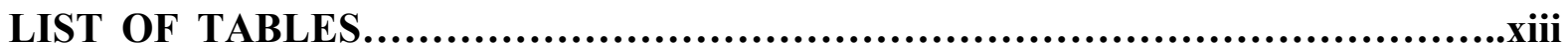

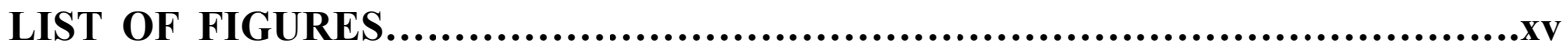

LIST OF ABBREVIATIONS AND SYMBOLS......................................xi

1. GENERAL INTRODUCTION..............................................

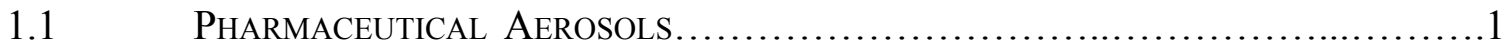

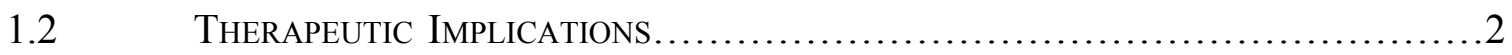

1.2.1 Disease Models: Asthma/COPD .................................2

1.2.2 Drugs and Receptors..........................................

1.2.3 Respiratory Deposition........................................

$1.3 \quad$ Dry POWDER INHALERS ................................................. 11

1.3.1 Aerosol Formulation...........................................13

1.3.2 DPI Device....................................................

$1.4 \quad$ Fundamental InTERMOlecular AND SURface ForCes.........................15

1.4.1 Adhesion and Cohesion Forces...................................15

1.4.2 Deaggregation (Separation) Forces.............................22

$1.5 \quad$ Arosolization Performance...................................................23

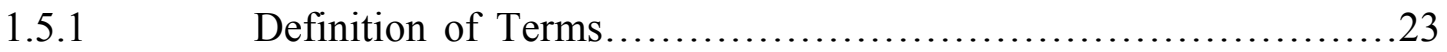

1.5.2 Aerosolization Performance....................................24

1.5.3 Current Trend for Performance Prediction..........................26

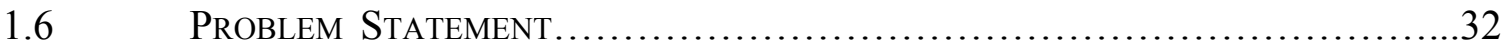


$1.7 \quad$ HypothesIS AND OBJectives............................................. 33

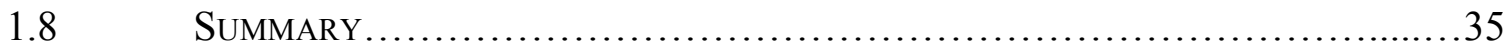

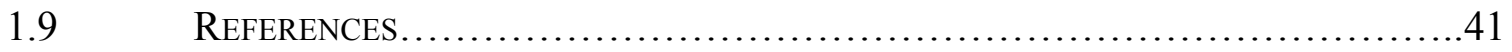

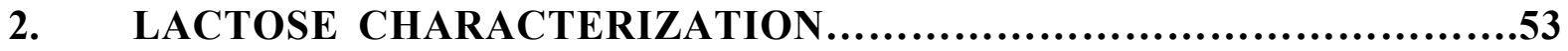

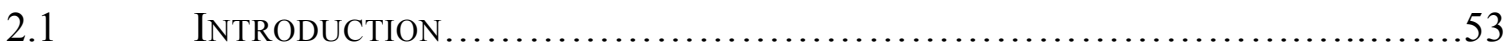

2.1.1 Lactose Monohydrate as Carrier...................................53

2.1.2 Techniques for Dry Powder Formulation Characterization..............56

2.2 Material and Methods....................................................61

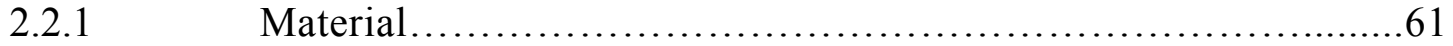

2.2.2 Blend Preparation....................................................61

2.2.3 Analytical Approaches for Lactose Batches and Blends...............62

2.2.4 Performance Assessment: TSLI; ACI..........................66

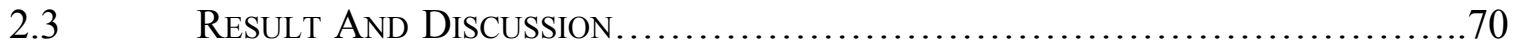

2.3.1 Aerosol Characterization..........................................70

2.3.2 In Vitro Aerosol Performance Assessment.............................75

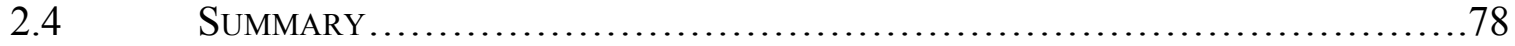

2.5 REFERENCES........................................................... 90

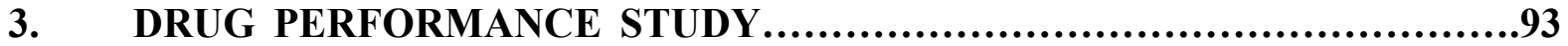

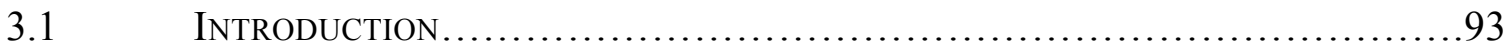

3.1.1 Drugs for the Treatment of Asthma/COPD.........................94

3.1.2 Physicochemical Properties....................................95

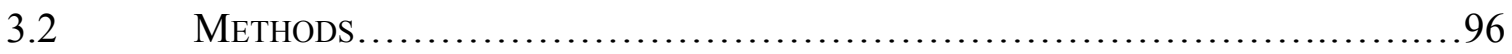

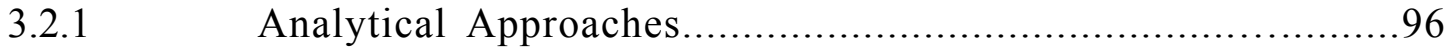


ReSUltS............................................................ 102

3.3.1 Aerosol Characterization................................... 102

In Vitro Aerosol Performance Assessment........................ 108

DiscusSION...................................................... 117

3.4.1 Influence of Drugs...................................... 117

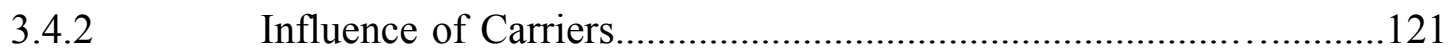

3.4.3 Influence of SET Shear Stress.................................123

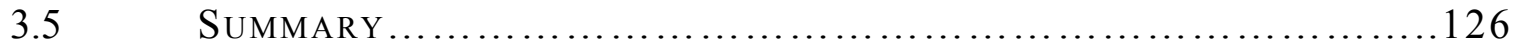

REFERENCES................................................... 144

4. ALTERNATIVE SUGARS AS CARRIER.................................147

INTRODUCTION................................................. 147

M.2 Material and Methods......................................... 151

4.2.1 Material...................................................... 151

4.2.2 Analytical Approaches..................................... 152

4.2.3 In Vitro Aerosol Performance Assessment.........................153

ResUlt......................................................... 153

4.3.1 Aerosol Characterization..................................... 153

4.3.2 In Vitro Aerosol Performance Assessment......................... 156

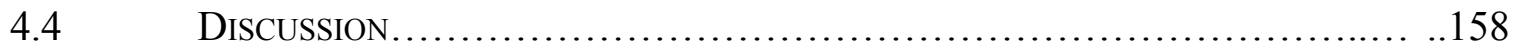

4.4.1 Influence of Drugs........................................

Influence of Carriers....................................... 159

4.4.3 Influence of SET Shear Stress............................... 160 


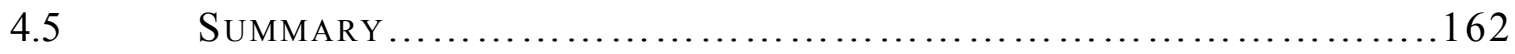

4.6 REFERENCES......................................................... 175

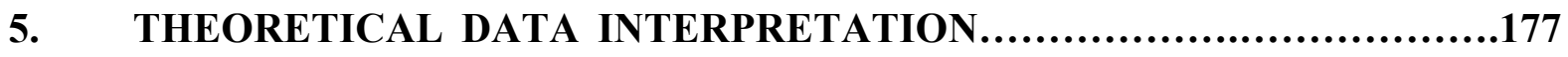

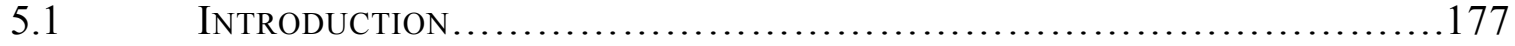

5.2 PADE-An Analogy to Langmuir Adsorption Isotherm............178

5.2.1 Surface Adsorption Theory..................................178

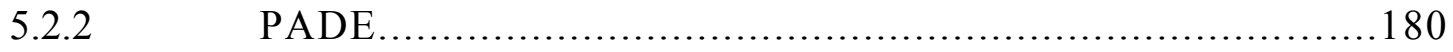

"EXPERIMENTAL" JUSTIFICATION OF PADE

5.3.1 The Different Linear Regression Models.......................183

5.3.2 The Adaptation of Different PADE Models...................... 185

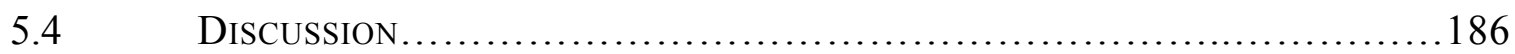

5.4.1 Similarites \& Differences Between Surface Adsorption \& Aerosol Deaggregation..........................................186

5.4.2 The Choice of Surface Adsorption Instead of Protein Binding.......190

5.4.3 PADE Considering Surface Heterogneity........................191

5.4.4 Application of the PADE................................... 194

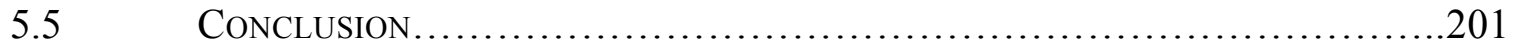

$5.6 \quad$ REFERENCES.................................................... 214

6. SURFACE MODIFICATION FOR LACTOSE...........................216

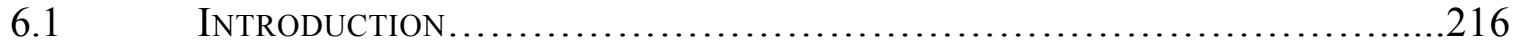

6.1.1 Proposed Ternary Blending Mechanisms......................216

6.1.2 The Nature of Particle Surfaces..............................221

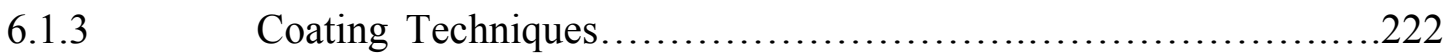

Methods.......................................................224 
6.2.1 Material.

6.2.2 Preparation of Fatty Acid Coated Lactose Monohydrate.............226

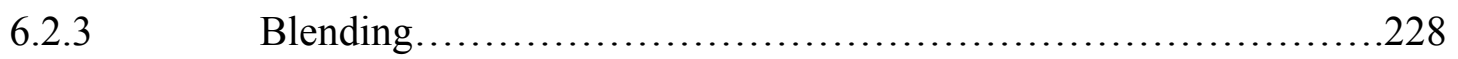

6.2.4 Physicochemical Characterization...........................229

6.2.5 Aerosolization Performance Assessment..........................230

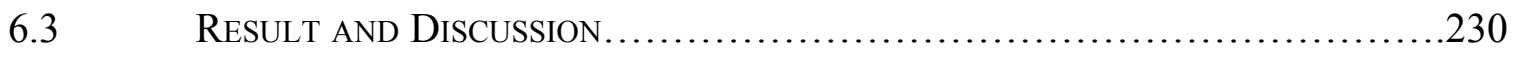

6.3.1 The Theoretical Estimation for Monolayer Surface Coating.........230

6.3.2 Fatty Acid Coating of Lactose Monohydrate......................231

6.3.3 Physicochemical Characterization..........................233

6.3.4 In Vitro Aerosolization Performance Assessment...................234

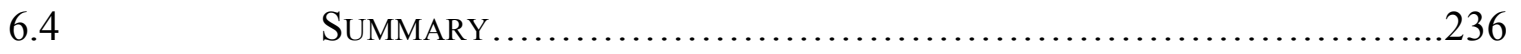

REFERENCE..............................................245

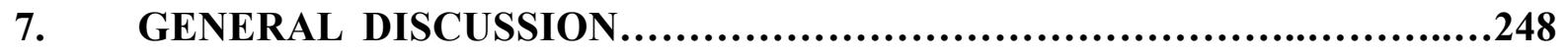

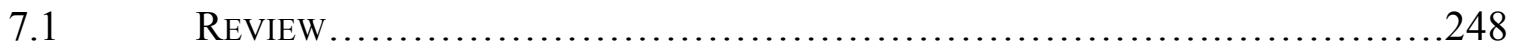

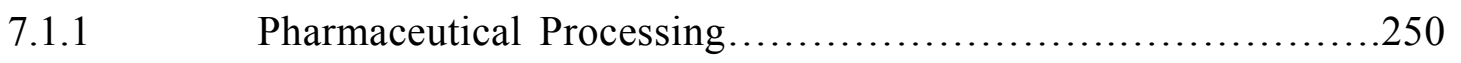

Summary of Findings........................................ 255

General Conclusion............................................260

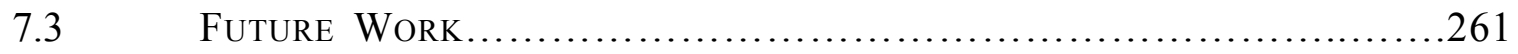

REFERENCES..................................................... 265

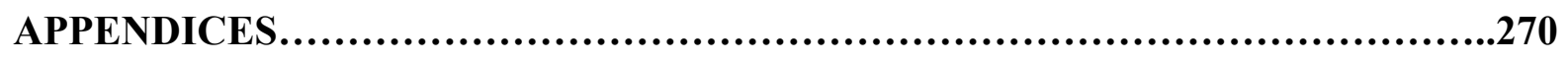

Appendix 1-A. Selected Dry Powder Inhalers (DPIs) and Their Characteristics........270

Appendix 2-A. TSLI Performance data...................................... 272

Appendix 2-B. Experimental Design of Albuterol Sulfate - Lactose Monohydrate Blends for Performance Study...............................273 
Appendix 2-C. Experimental Design of Albuterol Sulfate - Lactose Monohydrate Blends for Performance Study. 274

Appendix 3-A1. TSLI Performance Data of Albuterol Sulfate and Disodium Cromoglycate.

Appendix 3-A2. TSLI Performance Data of Ipratropium Bromide Monohydrate and Fluticasone Propionate...........................................276

Appendix 4-A. TSLI performance Data Using SET (2\%(w/w) drug conc.).............277

Appendix 5-A. The Schematic Calculation of Aerodynamic Parameters of Extra SETs. ....

Appendix 5-B. TSLI Performance Data: Aerosolization Performance Using Three Extra SETs. .281

Appendix 5-C. Schematic Calculation of Aerodynamic Shear Stress of DPI Devices: e.g. Aerolizer ${ }^{\circledR}$

Appendix 5-D. TSLI Performance Data: Aerosolization Performance Using Rotahaler ${ }^{\circledR}$ and Aerolizer ${ }^{\circledR}$ .283

Appendix 5-E. Performance Data of FP-Treh before and after Storage in the Desiccator

Appendix 6-A. The Amount of Fatty Acid Associated on the Surface of Lactose Monohydrate Powder Treated with Stearic Acid, Palmitic Acid, Myristic Acid, and Lauric Acid....

Appendix 6-B. The Aerosolization Performance after Stearic Acid Coating (AS Formulations). .286

Appendix 6-C. The Aerosolization Performance after Stearic Acid Coating (FP Formulations). .288 


\section{LIST OF TABLES}

Table 1.1 - SET Specifications (Dimensions and Airflow Parameters at Q $=60 \mathrm{~L} / \mathrm{min}$ )......36

Table 1.2 - Overview of Strategies that Control Drug-Carrier Interactions for In Vitro

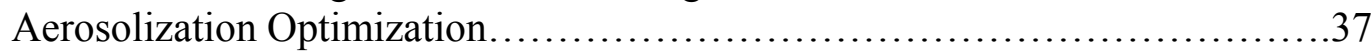

Table 2.1 - Selected Physicochemical Properties of Eight Batches of Lactose as Supplied by the Manufacturer.

Table 2.2 - The Eight-staged ACI Aerodynamic Cut-off Diameter $\phi(\mu \mathrm{m})$ at Airflow Rate $Q=60 \mathrm{~L} / \mathrm{min}$ for DPI Experiment after Calibration.

Table 3.1 - Particle Volume Size Distribution of Albuterol Sulfate, Disodium Cromoglycate, Ipratropium Bromide Monohydrate, and Fluticasone Propionate before and after Air Jet-milling. (Mean \pm SD).

Table 3.2 - TSLI Performance Data Analysis: The Relationship between SET Shear Stress $\left(\tau_{s}\right)$ and $F P F_{T D<6.4} / F P F_{E D<6.4}$ for IPB/Lactose Monohydrate and FP/Lactose Monohydrate Dry Powder Formulations Using Nonlinear Regression Analysis $(n=3)$.

Table 3.3 - ACI Drug Aerosolization Performance (ED, FPF, and MMAD) Studies via SET-D $\left(\tau_{s}=0.624 \mathrm{~N} / \mathrm{m}^{2}\right)$ and SET-A $\left(\tau_{s}=13.143 \mathrm{~N} / \mathrm{m}^{2}\right)$ at Airflow Rate $Q=60 \mathrm{~L} / \mathrm{min}$. The Formulations in Comparison Were SV-AS vs. ML-DSCG; and SV-IPB vs. ML-FP (Mean $\pm \mathrm{SD}, n=3$ )

Table 4.1 - Particle Volume Size Distribution of D-Mannitol and D-Trehalose Dihydrate (Sieved Fraction 45-75 $\mu \mathrm{m}$ )

Table 4.2 - TSLI Performance Data Analysis: The Relationship between SET Shear Stress $\left(\tau_{s}\right)$ and $F P F_{T D<6.4} / F P F_{E D<6.4}$ for Treh-based and Mann-based Dry Powder Formulations Using Nonlinear Regression Analysis $(n=3) \ldots \ldots \ldots \ldots .174$

Table 5.1 - Results of PADE Linear Regression Using Four Different Linearization Methods of PADE (Langmuir Isotherm Equivalent) for Performance Studies .203

Table 5.2 - A Comparison of Surface Adsorption, Molecular/Colloidal Assembly, and Aerosol Dispersion (Analogy and Contrast). 
Table 5.3 - A Schematic Comparison of Algebraic Equivalent Langmuir and Freundlich Expression for TSLI Formulation Performance (PADE Nonlinear Regression Analyses).

Table 5.4 - Dimensions of the Additional SETs and Their Corresponding Airflow Parameters

Table 5.5 - A Comparison of PADE Models Using Algebraic Equivalent Surface Adsorption Expressions for TSLI Formulation Performance (Using 7 SETs, Correlated by PADE nonlinear Regression Analyses Including Algebraic Equivalent Langmuir Isotherm, Freundlich, and Sips Expressions)

Table 6.1 - Physical Characterization of the Powder Flow Properties ( $\mathrm{n}=3$, Mean Values) 


\section{LIST OF FIGURES}

Figure 1.1 - Mechanisms of Action of Drugs for Asthma Therapy........................38

Figure 1.2 - Schematic Diagrams of the Dry Powder Inhalers: (A) Rotahaler, a unitdose low shear device; (B) Inhalator, a unit-dose high shear device; and

(C) The Various Transition Stages of Powder Aerosolization, through

Dilated, Free Flowing, and Finally Dispersed Powder..........................39

Figure 1.3 - The Magnitude of Interparticulate Forces: (A) Adhesion Forces; (B) Aerodynamic Deaggregation Forces

Figure 2.1 - The Specification of the Two Inertial Sampling Devices Used for Aerosolization Performance Studies: (A) Twin Stage Liquid Impinger (TSLI); (B) Andersen Cascade Impactor (ACI); (C) TSLI Collection Efficiency and Semi-empirical Oropharyngeal Deposition Curves (Model); (D) ACI Collection Efficiency Curves.

Figure 2.2 - Representative Scanning Electron Microscopy Images of Lactose Monohydrate Batches (A) SV-A; (B) SV-B; (C) SV-C; (D) SV-D; (E) SV-E; (F) SV-F; (G) ML-A; and (H) ML-B at 600× Magnification

Figure 2.3 - Representative Scanning Electron Microscopy Images of Micronized Albuterol Sulfate (AS): (A) AS at 5,000× Magnification and (B) AS at 20,000× Magnification; Lactose Monohydrate-based Blends (C) SV-B+ $0.5 \% \mathrm{w} / \mathrm{w}$ AS; (D) ML-A+2\%w/w AS; (E) SV-B+ $2 \% \mathrm{w} / \mathrm{w}$ AS; (F) SV$\mathrm{C}+2 \% \mathrm{w} / \mathrm{w}$ AS at $1,500 \times$ Magnification

Figure 2.4 - Representative Differential Scanning Calorimetry Thermograms of (A) SV-A and SV-B; (B) ML-A and ML-B; (C) and (D) Micronized Albuterol Ssulfate; (E) Blends of AS (2\%w/w) with SV-A and SV-B, Respectively; (F) Blends of AS $(2 \% \mathrm{w} / \mathrm{w})$ with ML-A and ML-B, Respectively....

Figure 2.5 - Representative X-ray Powder Height Intensity Diffractograms of Lactose Monohydrate (A) SV; (B) ML (Raw Data: Courtesy of Dr. Mansour. The Data Were Re-plotted Using Match ${ }^{\circledR}$ Software, Crystal Impact, Inc.); (C) The University of Cambridge Database of Pure $\alpha$ Lactose Monohydrate; (D) The University of Cambridge Database of Pure $\beta$-lactose.

Figure 2.6 - Representative Atomic Force Microscopy Topographic Images of Lactose Monohydrate by Tapping Mode: (A) SV-B $(10 \mu \mathrm{m} \times 10 \mu \mathrm{m})$; 
(B) SV-C $(10 \mu \mathrm{m} \times 10 \mu \mathrm{m})$; (C) ML-B $(10 \mu \mathrm{m} \times 10 \mu \mathrm{m})$; (D) ML-B

$(50 \mu \mathrm{m} \times 50 \mu \mathrm{m})$; and $(\mathrm{E}-\mathrm{F}) \mathrm{SV}-\mathrm{F}(5 \mu \mathrm{m} \times 5 \mu \mathrm{m})$.

Figure 2.7 - The Results of TSLI Performance Study of Intereactive Physical Mixtures: Albuterol Sulfate (AS) and Lactose Monohydrate (SV131 and ML058). Two Concentrations of AS Were Prepared at $0.5 \% \mathrm{w} / \mathrm{w}$ and $2 \% \mathrm{w} / \mathrm{w}$. The Performance Results (ED and $\mathrm{FPF}_{\mathrm{TD}}$ ) Were Plotted Against SET Parameters (Pressure Drop, Reynolds Number, and Shear Stress).

Figure 2.8 - The Results of ACI Performance Study of Intereactive Physical Mixtures: Albuterol Sulfate (AS) and Lactose Monohydrate (SV131 and ML058):

(A) ED of $0.5 \% \mathrm{w} / \mathrm{w}$ AS-SV131; (B) ED of 2\%w/w AS-ML058; (C) $\mathrm{FPF}_{\mathrm{TD}}$ of $0.5 \% \mathrm{w} / \mathrm{w}$ AS-SV131; (D) $\mathrm{FPF}_{\mathrm{TD}}$ of $2 \% \mathrm{w} / \mathrm{w}$ AS0-ML058; (E) MMAD When Solenoid Switch Was Operated Off; (F) MMAD When Solenoid Switch On.

Figure 3.1 - Chemical Structures of (A) Albuterol Sulfate; (B) Disodium Cromoglycate;

(C) Ipratropium Bromide Monohydrate; (D) Fluticasone Propionate; (E) $\alpha$-Lactose Monohydrate.

Figure 2.4 - Representative Scanning Electron Micrographs of Disodium Cromoglycate (DSCG), Ipratropium Bromide Monohydrate (IPB), and Fluticasone Propionate (FP) before and after Micronization by Air Jet-milling:

(A) Raw DSCG (3k $\times$ Magnification); (B) Micronized DSCG (20k $\times$ );

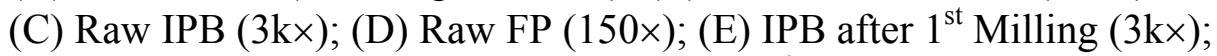

(F) FP after $1^{\text {st }}$ Milling $(3 \mathrm{k} \times)$; (G) IPB after $2^{\text {nd }}$ Milling $(10 \mathrm{k} \times)$; and $(\mathrm{H})$ FP after $2^{\text {nd }}$ Milling $(10 \mathrm{k} \times)$.

Figure 3.3 - Representative SEM Images of Drugs (DSCG, IPB, FP) and Lactose Monohydrate (2 sieved batches (SV) and 2 milled batches (ML)) Interactive Physical Mixtures at 2\%w/w Drug Concentration: (A) DSCG-SV (1,500×); (B) DSCG-ML (1,500×); (C) IPB-SV (1,500×); (D) IPB-ML (1,500×); (E) FP-SV (600×); and (F) FP-ML $(600 \times)$

Figure 3.4 - Representative Differential Scanning Calorimetry Thermograms of Raw Drugs and Drugs After Micronization by Jet-milling: (A) Unmilled and micronized DSCG; (B) Unmilled and micronized IPB; (C) Unmilled and micronized FP. Scanning rate is at $5{ }^{\circ} \mathrm{C} / \mathrm{min}$. .135

Figure 3.5 - Representative DSC Thermograms of Drug-Lactose Monohydrate (SVs or MLs) Interactive Physical Mixtures: (A) Blends of DSCG $(2 \% \mathrm{w} / \mathrm{w})$ with SV-A and SV-B, Respectively; (B) Blends of DSCG $(2 \% \mathrm{w} / \mathrm{w})$ with ML-A and ML-B, Respectively; (C) Blends of IPB 
(2\%w/w) with SV-A and SV-B, Respectively; (D) Blends of IPB

$(2 \% \mathrm{w} / \mathrm{w})$ with ML-A and ML-B, Respectively; (E) Blends of FP

$(2 \% \mathrm{w} / \mathrm{w})$ with SV-A and SV-B, respectively; and (F) Blends of FP

$(2 \% \mathrm{w} / \mathrm{w})$ with ML-A and ML-B, respectively. Scanning Rate Is At

$5{ }^{\circ} \mathrm{C} / \mathrm{min}$

Figure 3.6 - X-ray Powder \%Height Intensity Diffractograms of Micronized Drugs

(A) Albuterol Sulfate; (B) Disodium Cromoglycate; (C) Ipratropium

Bromide Monohydrate; and (D) Fluticasone Propionate.

Figure 3.7 - The Relationship between SET Airflow Parameter (Exemplified by Shear Stress $\tau_{\mathrm{s}}$ ) and TSLI Formulation Performance $\left(E D, F P F_{T D}, F P F_{E D}\right)$.

Nonlinear Regression Analyses Were Applied Using PADE for $F P F-\tau_{s}$ Correlation. Left panels (A) to (C) are AS/Lactose Monohydrate Systems (2\%w/w): (A) $E D-\tau_{s}$; (B) $F P F_{T D^{-}} \tau_{s}$ Curve Fitting; (C) $F P F_{E D^{-}} \tau_{s}$ Curve Fitting. Right Panels (D) to (F) Are DSCG/Lactose Monohydrate Systems (2\%w/w): (D) $E D-\tau_{s}$; (E) $F P F_{T D^{-}} \tau_{s}$ Curve Fitting; (F) $F P F_{E D^{-}} \tau_{s}$ Curve

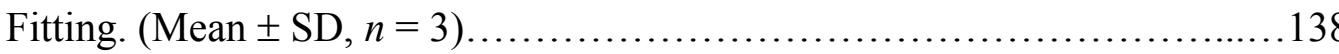

Figure 3.8 - The Relationship between SET Airflow Parameter (Exemplified by Shear Stress $\tau_{\mathrm{s}}$ ) and TSLI Formulation Performance (Exemplified by $\left.F P F_{T D}\right)$. Linear Regression Analyses Were Applied Using Langmuir Linear Regression. (Eq. 3-2). (A) 2\% (w/w) AS/Lactose Monohydrate Systems; and (B) 2\% (w/w) DSCG/Lactose Monohydrate Systems. The $R^{2}$ Represents the Goodness of Fit of Regression Lines. (Mean $\pm \mathrm{SD}, n=3$ )

Figure 3.9 - The relationship between SET Shear Stress $\left(\tau_{\mathrm{s}}\right)$ and TSLI Formulation Performance $\left(E D, F P F_{T D}, F P F_{E D}\right)$. Nonlinear Regression Analyses Were Applied Using PADE for $F P F-\tau_{s}$ Correlation (Eq.3-1). Left Panels (A) to (C) are IPB/Lactose Monohydrate Systems ( $2 \% \mathrm{w} / \mathrm{w}):(\mathrm{A}) E D-\tau_{s}$; (B) $F P F_{T D^{-}} \tau_{s}$ Curve Fitting; (C) $F P F_{E D^{-}} \tau_{s}$ Curve Fitting. Right Panels (D) to (F) Are FP/Lactose Monohydrate Systems ( $2 \% \mathrm{w} / \mathrm{w}):(\mathrm{D}) E D-\tau_{s}$; (E) $F P F_{T D^{-}} \tau_{s}$ Curve Fitting; (F) $F P F_{E D^{-}} \tau_{s}$ Curve Fitting. (Mean $\pm \mathrm{SD}, n=3$ ).....140

Figure 3.10 - The Relationship between TSLI Formulation Performance (Exemplified by $\left.F P F_{T D}\right)$ and SET Shear Stress $\left(\tau_{s}\right)$. Linear Regression Analyses Were Applied Using PADE Linear Regression (Eq. 3-2). (A) 2\%w/w IPB/ Lactose Monohydrate Systems; and (B) 2\%w/w FP/Lactose Monohydrate Systems. The $R^{2}$ Represents the Goodness of Fit of Regression Lines. (Mean $\pm \mathrm{SD}, n=3$ ).

Figure 3.11 - The ACI Deposition Pattern of AS/SV, DSCG/ML, IPB/SV, and FP/ML Formulations Using SETs (Device, Throat, and Preseparator 
Drug Deposition Were Included in Stage Deposition Calculation but not Plotted). (A) $2 \%(\mathrm{w} / \mathrm{w}) \mathrm{AS} / \mathrm{SV}$ at lowest shear stress (SET-D, $\tau_{s}=$ $\left.0.624 \mathrm{~N} / \mathrm{m}^{2}\right)$ vs. $\%(\mathrm{w} / \mathrm{w}) \mathrm{DSCG} / \mathrm{ML}$ at highest shear stress (SET-A, $\left.\tau_{s}=13.143 \mathrm{~N} / \mathrm{m}^{2}\right)$; (B) $2 \%(\mathrm{w} / \mathrm{w}) \mathrm{AS} / \mathrm{SV}$ at highest shear stress $v s$. $2 \%(\mathrm{w} / \mathrm{w}) \mathrm{DSCG} / \mathrm{ML}$ at lowest shear stress (SET-A, $\tau_{s}=13.143 \mathrm{~N} / \mathrm{m}^{2}$ ); (C) $2 \%(\mathrm{w} / \mathrm{w}) \mathrm{IPB} / \mathrm{SV}$ at lowest shear stress $v s .2 \%(\mathrm{w} / \mathrm{w}) \mathrm{FP} / \mathrm{ML}$ at highest shear stress; (D) 2\% (w/w) IPB/SV at highest shear stress $v s$. $2 \%(\mathrm{w} / \mathrm{w}) \mathrm{FP} / \mathrm{ML}$ at lowest shear stress. (Mean $\pm \mathrm{SD}, n=3$ ).

Figure 3.12 - ACI Performance Evaluation $\left(F P F_{T D<6.5}\right)$ by Dual-point Performance Evaluation, FPF and shear stress (SET-D, $\tau_{s}=0.624 \mathrm{~N} / \mathrm{m}^{2}$ and SET-A, $\tau_{s}=13.143 \mathrm{~N} / \mathrm{m}^{2}$ ) correlation: (A) $2 \%(\mathrm{w} / \mathrm{w}) \mathrm{AS} / \mathrm{SV}$ vs. $2 \%(\mathrm{w} / \mathrm{w})$ DSCG/ML; and (B) 2\% (w/w) IPB/SV vs. 2\% (w/w) FP/ML (Mean \pm $\mathrm{SD}, n=3)$

Figure 4.1 - Schematic Representation of Potential Sugars and Sugar Alcohols Explored for Dry Powder Formulation Carriers. (A) D-Glucose; (B) DTrehalose; (C) D-Maltose; (D) D-Raffinose; (E) Erythritol; (F) D-Xylitol; (G) D-Mannitol; (H) D-Sorbitol; (I) D-Maltitol. .164

Figure 4.2 - Representative Scanning Electron Micrographs (SEM) of Sugars $(1,500 \times$ magnification $)$ and Interactive Physical Mixtures $(600 \times)$ of Micronized Pulmonary Drug/Sugar Carrier Systems in the Solid-state: (A) D-Trehalose Dihydrate; (B) D-Mannitol; (C) Albuterol Sulfate/DTrehalose Dihydrate; (D) Albuterol Sulfate/D-Mannitol. 165

Figure 4.3 - Representative Scanning Electron Micrographs (SEM) of Interactive Physical Mixtures $(600 \times)$ of Micronized Pulmonary Drug/Sugar Carrier Systems in the Solid-state: (A) Disodium Cromoglycate/D-Trehalose Dihydrate; (B) Disodium Cromoglycate/D-Mannitol; (C) Ipratropium Bromide Monohydrate/D-Trehalose Dihydrate; and (D) Ipratropium Bromide Monohydrate /D-Mannitol. .166

Figure 4.4 - Representative Differential Scanning Calorimetry (DSC) Thermograms at a Heating Rate of $5{ }^{\circ} \mathrm{C} / \mathrm{min}$ for Carriers: D-Mannitol (Mann) and DTrehalose Dihydrate (Treh) in Solid State: (A) Mann; (B) Treh (The $1^{\text {st }}$ scan: $5{ }^{\circ} \mathrm{C} / \mathrm{min}$ up to $220{ }^{\circ} \mathrm{C}$; the $2^{\text {nd }}$ scan: $\left.20{ }^{\circ} \mathrm{C} / \mathrm{min}\right)$

Figure 4.5 - Representative DSC Thermograms at a Heating Rate of $5{ }^{\circ} \mathrm{C} / \mathrm{min}$ for Interactive Physical Mixtures. Drugs: AS, DSCG, IPB, and FP; Carriers: Mann and Treh (sieved fraction: 45-75 $\mu \mathrm{m}$ ): (A). AS/Mann; (B). AS/Treh; (C). DSCG/Mann; (D). DSCG/Treh; (E). IPB/Mann; (F). IPB/Treh; (G). FP/Mann; and (H) FP/Treh... 
Figure 4.6 - X-ray Powder (slit-detector) Height Intensity Diffractograms* of Alternative Sugar Carriers (Sieved Fraction: $45-75 \mu \mathrm{m}$ ) at a Scanning Rate of $2 \theta$ degrees/minute: (A) Mann; (B) Treh.

Figure 4.7 - PADE Nonlinear Regression Applied to TSLI Aerosolization ( $\mathrm{FPF}_{\mathrm{TD}<6.4 \text { : }}$ Mean \pm SD, $n=3$ ) of Dry Powders for Various Interactive Physical Mixtures of micronized pulmonary drugs with trehalose dihydrate and D-mannitol Sugar Carrier Systems: (A) AS; (B) IPB; (C) DSCG; and (D) FP.

Figure 4.8 - PADE Linear Regression Applied to TSLI Aerosolization $\mathrm{FPF}_{\mathrm{TD}<6.4}$ : Mean Value, $n=3$ ) of Dry Powders for Various Interactive Physical Mixtures of Micronized Pulmonary Drugs with Treh and Mann Carrier Systems: (A) AS; (B) IPB; (C) DSCG; and (D) FP.

Figure 4.9 - The Relationship Between SET Shear Stress $\left(\tau_{s}\right)$ and TSLI Formulation Performance $(E D)$. Various Interactive Physical Mixtures of Micronized Pulmonary Drugs with Treh and Mann carrier systems: (A) AS; (B) IPB;

(C) DSCG; and (D) FP............................................. 172

Figure 5.1 - PADE (Langmuir Equivalent Expression) and Its Linear Transformation......208

Figure 5.2 - The Relationship between $F P F_{T D}$ and SET Shear stress $\left(\tau_{s}\right)$ by TSLI Experiment. PADE Linear Regression Analyses: (A) AS formulations; (B) DSCG formulations; (C) IPB formulations; (D) FP formulations. All Formulations Were at $2 \% \mathrm{w} / \mathrm{w}$ Drug Concentration. .209

Figure 5.3 - TSLI Experimental Dataset of (A) DSCG/ML-B and (B) AS/Mann Formulations Using Additional SETs, and Correlation Using PADE Nonlinear Regression: Algebraic Equivalent of Surface Adsorption Expressions Including Langmuir, Freundlich, and Sips Equivalent. .210

Figure 5.4 - Schematic Illustration of Different Models of Heterogeneous Surfaces in Adsorption Theory. .211

Figure 5.5 - TSLI Performance $\left(F P F_{T D}\right)$ and Shear Stress $\left(\tau_{s}\right)$ Correlation: $(\mathrm{A})$ Performance Predicted by PADE Non-linear Regression; (B) Performance Predicted by PADE Linear Regression 212

Figure 5.6 - The Influence of Formulation Performance upon Storage: (A) DSCGML780, Nonlinear Regression; (B) DSCG-ML780, Linear Regression; (C) FP-Treh, Nonlinear Regression; (D) FP-Treh, Linear Regression. 
Figure 6.1 - Proposed Deaggregation Mechanisms of Ternary Blends....

Figure 6.2 - Representative Gas Chromatography Standard Peaks and Retention Time.....239

Figure 6.3 - Adsorption-association Isotherm of Fatty Acids with SV and ML Lactose Monohydrate. (A) Stearic Acid; (B) Palmitic Acid; (C) Myristic Acid; and (D) Lauric Acid................................................240

Figure 6.4 - DSC Thermograms of Lactose Monohydrate after Fatty Acid Surface Coating: (A) -SVcontrol $\left(\mathrm{CH}_{2} \mathrm{Cl}_{2} / \mathrm{CHCl}_{3}\right.$ treated); SVlow $(\sim 6 \mu \mathrm{g} / \mathrm{g}$ Stearic Acid); SVhigh ( $140 \mu \mathrm{g} / \mathrm{g}$ stearic acid); (B) -MLcontrol $\left(\mathrm{CH}_{2} \mathrm{Cl}_{2} / \mathrm{CHCl}_{3}\right.$ Treated); MLlow; MLhigh; (C) SV-AS; (D) ML-AS; (E) SV-FP; (F) ML-FP.

Figure 6.5 - PADE Nonlinear Regression and Linear Regression of AS Formulations......242

Figure 6.6 - PADE Nonlinear Regression and Linear Regression of FP Formulations.....243

Figure 7.1 - Pharmaceutical Dry Powder Aerosol Processing and Variables Associated with Each Process............................................264 


\section{LIST OF ABBREVIATIONS AND SYMBOL}

AS

$\mathrm{ACI}$

AFM

COPD Chronic Obstructive Pulmonary Disease

$d_{50}$

$D_{10}$

$D_{50}$

$D_{90}$

$D_{a e}$

$\Delta P$

DSC

DSCG

DPI

FP

FPF

FPM

GSD

Albuterol Sulfate

Andersen non-viable Cascade Impaction

Atomic Force Microscopy collection efficiency of an impactor

Pressure drop

Differential Scanning Calorimetry

Disodium Cromoglycate

Dry Powder Inhaler

Fluticasone Propionate

Geometric Standard Deviation
Cutoff diameter, cutsize, or cutoff, particle aerodynamic diameter having $50 \%$

Diameter which $10 \%$ of particles are smaller than

Diameter which $50 \%$ of particles are smaller than

Diameter which $90 \%$ of particles are smaller than

Aerodynamic diameter; $D_{a e}=D \sqrt{\rho}$, assuming spherical particle, $D$ is the volume diameter and $\rho$ the true density of particle.

Fine Particle Fraction; The proportion with respect to the nominal dose in a size range considered sufficiently small to enter the lungs

Fine Particle Mass; The mass with respect to the nominal dose in a size range considered sufficiently small to enter the lungs 


\begin{tabular}{|c|c|}
\hline HPMC & Hydroxypropyl Methylcellulose \\
\hline IGC & Inverse Gas Chromatography \\
\hline Mann & D-Mannitol \\
\hline ML & Milled Lactose Monohydrate \\
\hline ML058 & Milled Lactose Monohydrate Batch 058 or ML-A \\
\hline ML780 & Milled Lactose Monohydrate Batch 780 or ML-B \\
\hline MMAD & $\begin{array}{l}\text { Mass Median Aerodynamic Diameter; The aerodynamic particle size for } \\
\text { which } 50 \% \text { of the total aerosol by mass has smaller aerodynamic diameters }\end{array}$ \\
\hline IPB & Ipratropium Bromide Monohydrate \\
\hline PADE & Powder Aerosol Deaggregation Equation \\
\hline pMDI & Pressurized Metered-Dose Inhaler \\
\hline$Q$ & Flow rate of the air \\
\hline $\operatorname{Re}$ & Reynolds number \\
\hline$R_{D}$ & Specific resistance \\
\hline RH & Relative humidity \\
\hline $\mathrm{RH}_{0}$ & Critical relative humidity \\
\hline SEM & Scanning Electron Microscopy \\
\hline SET & Standardized Entrainment Tube \\
\hline Span & $\begin{array}{l}\text { A dimensionless measure of the breadth and uniformity of a distribution, } \\
\text { Span }=\frac{D_{90}-D_{10}}{D_{50}} \times 100 \%\end{array}$ \\
\hline
\end{tabular}

SV Sieved Lactose Monohydrate 
SV208 Sieved Lactose Monohydrate Batch 208 or SV-A

SV473 Sieved Lactose Monohydrate Batch 473 or SV-B

$\tau_{s}$

Shear stress

Treh Trehalose Dihydrate

TSLI Twin-Stage Liquid Impinger

VMD Volume Median Diameter

XRPD X-ray Powder Diffraction 


\section{CHAPTER 1 GENERAL INTRODUCTION}

\subsection{Pharmaceutical Aerosols}

An aerosol is defined as a collection of solid or liquid particles suspended in a gas. Therapeutic aerosols are directly delivered into the respiratory tract for local or systemic action. Of the various pharmaceutical dosage forms and routes of administration, the delivery of aerosols by inhalation frequently offers advantages with respect to oral or parenteral routes (Figure 1-1).[1] Briefly, it is a method of non-invasive and local delivery, which may improve efficacy and reduce unwanted systemic side effect. The large surface area and thin alveolar epithelium of the lungs allow rapid absorption and onset of action to the target site. The absence of first-pass metabolism ensures high bioavailability compared to other route of administration.

There is a long history of anecdotal inhalation therapy that can be traced back $4 \sim 5$

thousand years. However, the commercialization of inhalation products with manufacturing standards and stringent test procedure occurred only recently.[2] During the development of modern inhalation therapy, three distinct categories of delivery systems including nebulizer, propellant driven metered dose inhaler (pMDI), and dry powder inhaler (DPI) evolved.[3] Among them, the nebulizer has the longest history of use for delivery of therapeutic agents.[4] pMDIs and DPIs emerged for the treatment of asthma in the mid-1950s and 1960s, respectively.[5,6] More recently there has been an increased interest in the development of DPIs due to the phase-out of ozone depleting propellants used in pMDIs.[7, 8] Delivery of 
small molecular weight drugs for the treatment of diseases such as asthma and chronic obstructive pulmonary disease (COPD) is the major application for this technology.[9] The drugs used for these purpose are mainly classified as $\beta_{2}$-adrenergic receptor agonists, mast cell stabilizers, cholinergic receptor antagonists, and corticosteroids.[6] The pulmonary drug delivery is also being used for respiratory tract and lung infections using antibiotics or vaccines, or the delivery of protein, peptides and macromolecules for local and systemic diseases.[10, 11]

Asthma therapy is the major application of drugs in these therapeutic categories. These drugs may delivered directly to the lungs to treat the cause and symptoms of disease which are outlined in the following section

\subsection{Therapeutic Implications}

\section{$\underline{1.2 .1 \text { Asthma }}$}

Asthma is a chronic inflammatory airway disease. It is characterized by bronchoconstriction and inflammation of the airways, which is partially or completely reversible either spontaneously or with treatment. Acute exacerbations of asthma result in symptoms including wheezing, cough, and dyspnea. However, limited symptoms associate with asthma between acute exacerbations and even pulmonary function tests may be normal. Whereas past definitions emphasized obstruction, more accurate definition focuses on asthma as a primary inflammatory disease which is the major cause of bronchial hyperreactivity and airflow obstruction.[12] Approximately 7.2\% of U.S. adults have asthma and in 2006 accounted for 3613 deaths in the United States.[13] Asthma can be triggered by airway 
inflammation caused by allergens, respiratory infections, occupational and environmental stimuli, cigarette smoke, pharmacological agents such as methacholine, histamine, and aspirin along with non-steroidal anti-inflammatory agents, and many unknown inflammatory mediators.[14, 15] Asthma often occurs at night (Nocturnal asthma) because of sleepinduced airway smooth muscle tone change and reduced circulating catecholamine levels. Inflammation also causes an associated increase in bronchial hyperresponsiveness to stimuli. Mucus hypersecretion can occur in acute and chronic feature of asthma accompanying airway inflammation, which, in severe asthma, lead to fatal airway obstruction.[14] Asthma is mediated by antibodies (IgE) found on mast cells in the airway mucosa. On exposure to antigens, there is an increase in CD4+ $\mathrm{T}$ lymphocytes that orchestrate the degranulation of mast cells to release primary mediators including histamine, eosinophilic, and neutrophilic chemotactic factors, which cause rapid and episodic bronchoconstriction and increased vascular permeability. In asthma, cytokines such as interleukin (IL)-4, IL-5, and IL-13 may be important, because they are necessary for $\operatorname{IgE}$ formation and eosinophilic inflammation.[14] The secondary mediators including leukotrienes and prostaglandins may result in prolonged bronchoconstrictor response.[16] Several inflammatory enzymes such as mast cell tryptase may play important role in hyperreactivity and in some aspects of airway remodeling in asthma.[17]

\subsubsection{Drugs and Receptors}

Pharmacological control of the airway is mediated by receptors found on bronchial airway and vessel smooth muscle, secretory cells, bronchial epithelium, and pulmonary and bronchial blood vessels.[18] The receptors for $\beta$-adrenergic agonists are distributed 
throughout the lung, but are more accumulated in the periphery of respiratory systems. Both sympathetic (adrenergic) and parasympathetic (cholinergic) receptors control the lung airflow. The sympathetic postganglionic fibers primarily secrete norepinephrine. The adrenal glands release epinephrine when they receive sympathetic signals from the spinal cord. Both epinephrine and norepinephrine bind to $\alpha$-adrenergic receptors of blood vessels to cause constriction and to $\beta$-adrenergic receptors of the bronchial airway and vessel smooth muscle to cause dilation of the airways and blood vessels. The parasympathetic postganglionic fibers secrete acetylcholine as primary neutrotransmitter. Acetylcholine binds to $M_{3}$ muscarinic cholinergic receptors and cause airway smooth muscle constriction, blood vessel dilation, and glandular secretion.

Classes of drugs commonly prescribed for asthma therapy include: 1) Bronchodilators (i.e. drugs that result in bronchodilation of the airway smooth muscle) such as $\beta$-adrenergic agonists, anticholinergics, and phosphodiesterase inhibitors (xanthine derivatives); 2) Drugs that reduce or control of inflammatory mediator release/synthesis such as corticosteroid; 3) Cromone derivatives that prevent mast cell degranulation. A schematic representation of the various pathways leading to bronchoconstriction, and the classes of inhaled anti-asthmatic drugs that are utilized to act at specific stages are shown in Figure 1-1.[19] These therapeutic agents are mainly aerosolized and delivered by inhalation to respiratory tract.

\subsubsection{1 $\beta$-Adrenergic agonists}

$\beta$-Adrenergic agonists are a group of catecholamine derivatives that stimulate sympathetic nervous system and allow relaxation of airway smooth muscle in the presence of reversible airflow obstruction. The trend in this type of bronchodilators has been toward development 
from nonspecific (such as epinephrine) to $\beta_{2}$ specific agents, in order to reduce side-effect exerted by stimulation of undesired adrenergic receptors (such as cardiac effect). $\beta_{2}$-agonists are a primary group of bronchodilators used in asthma treatment. Based on the duration of action in the lung following delivery, they are classified as very short-acting such as isoetharine; short-acting such as albuterol (GlaxoSmithKline, 3M, Schering Plough), terbutaline (AstraZeneca), and fenoterol (Boehringer Ingelheim); and long-acting bronchodilators such as salmeterol (GlaxoSmithKline) and formoterol (Boehringer Ingelheim).[19] Treatment with regular $\beta$-agonist therapy in severe asthma does not address the underlying inflammatory process. Quite a few studies have implicated the use of shortacting $\beta$-agonist causes increased hyperreactivity, worsening clinical outcomes, and mortality, which is known as asthma paradox.[20] Co-administration with anticholinergics or corticosteroids could achieve additive bronchodilation or anti-inflammation effect.

\subsubsection{Anticholinergics}

This class of drugs induce bronchodilation and inhibit mucus secretion by competitive inhibition of cholinergic receptors, causing blockade of acetylcholine-induced stimulation of guanyl cyclase, which, in turn, reduces the formation of cyclic guanosine monophosphate (cGMP), a mediator of bronchoconstriction.[21] They are bronchodilators for treatment of asthma. The representative drugs include ipratropium (Boehringer Ingelheim), a nonselective, short-acting bronchodilator; oxitropium; and tiotropium (Boehringer Ingelheim). Ipratropium has been co-administered with albuterol (Combivent $\left.{ }^{\circledR}\right)$ to achieve additive bronchodilator effect and prevent $\beta_{2}$-agonist induced bronchospasm.[22]

\subsubsection{Corticosteroids}


Corticosteroids are the most effective medication currently available for the treatment of asthma. Although their exact mechanism of action is still unclear, they are effectively used for suppressing airway inflammation that usually decrease bronchial hyperreactivity and airflow obstruction associated with asthma.[23] They also inhibit prostaglandins and leukotrienes, as well as cyclooxygenases (both COX-1 and COX-2) expression, providing long-term control of persistent asthma symptoms. The systemic use of corticosteroids causes significant systemic immunosuppression, while local delivery with aerosol corticosteroids has far fewer side effects. This class of drugs include beclomethasone dipropionate (GlaxoSmithKline, 3M, and Schering Plough), triamcinolone acetonide (Aventis), budesonide (AstraZeneca), flunisolide, fluticasone (GSK), and Mometasone furoate (Schering Plough).[19]

\subsubsection{Cromone derivatives}

Cromones are a unique class of compounds that prevent the onset and development of asthma through inhibition of chemical mediators of inflammation. The representative drug, disodium cromoglycate (Aventis), was formulated as the first marketed dry powder inhalation product for the treatment of asthma.[2] The mechanism of action of this class of drugs has yet to be fully elucidated. Generally, they are mast cell stabilizers that prevent histamine release involved in local hypersensitivity of the lungs.[19]

\subsubsection{Xanthine derivatives}

Xanthine derivatives such as theophylline have the effect of diuresis and smooth muscle relaxation.[24] They are used as bronchodilator for the treatment of both asthma and stable state COPD. The exact mechanism of action of xanthines is still debatable. It is generally 
proposed that xanthines cause smooth muscle relaxation by inhibition of phosphodiesterase, leading to an increase in intracellular cyclic adenosine $3^{\prime}, 5^{\prime}$-monophosphate (cAMP). An increase in cAMP results in bronchial smooth muscle relaxation.[24]. Xanthine derivitives are not delivered as aerosols but are included as this completes the pharmacology of locally acting agents in the lungs.

Once the pharmacological targets have been identified delivering the drugs to the site of action requires consideration of the anatomy and physiology of the lungs.

\subsubsection{Respiratory Deposition}

Airflow in the respiratory system is complex. The understanding of the local aerodynamics derives mainly from experimental studies using physical models of lung airways. The respiratory system can be divided into three regions: the head airway (nasopharyngeal) region, tracheobronchial region, and alveolar region.[25] Each region covers several anatomical units exhibiting distinct difference in structure, airflow pattern, and function. In Weibel's model, 23 generations of the airway from trachea to alveolar sacs were designated.[26] An estimate of an average adult's airflow indicates that flows in the upper airway are turbulent to transition region, while the 16 generation and below are laminar flow, as is indicated by the Reynolds number $(R e)$ of the airway.[26] For the majority of pharmaceutical aerosols, the dynamics of particles in the airways fall in the Stokes' regime for particle diameters larger than $1.3 \mu \mathrm{m}$.[27] Particle movement in this size regime follows Stokes's law (Eq. 1-1). The individual curvilinear particle motion can be characterized including the terminal settling velocity $\left(V_{T S}\right)$, relaxation time $(\tau)$, and Reynolds number of 
particles $\left(R e_{p}\right)$.[28] Equating the aerodynamic drag force with the Stokes's law result in the terminal settling velocity in a laminar condition and neglecting slip correction:[25]

$$
\begin{gathered}
F_{D}=3 \pi \eta V d \quad \text { (Eq. 1-1) } \\
V_{T S}=\frac{\rho_{p} d_{e}^{2} g}{18 \eta \chi} \quad(\text { for } \mathrm{Re}<1) \quad(\mathrm{E})
\end{gathered}
$$

where $\rho_{p}$ is the particle density; $d_{e}$ is the equivalent volume diameter (diameter of a sphere having the same volume as that of the irregular particle); $\eta$ is the dynamic viscosity of air $\left(1.81 \times 10^{-5} \mathrm{~kg} /(\mathrm{ms})\right.$ at $\left.20{ }^{\circ} \mathrm{C}\right) ; \chi$ is the dynamic shape factor. Reynolds number of particle is defined as:

$$
\operatorname{Re}_{p}=\frac{\rho_{p} d_{p} V}{\eta} \quad \text { (Eq. 1-3) }
$$

where $d_{p}$ is the particle diameter, $V$ is the relative velocity between particle and airflow.

The airflow in the lower respiratory tract and alveolar region is generally laminar. Turbulent deposition may happen in the upper respiratory tract, but will not be discussed here. Particles dispersed in airflow follow curvilinear motion. The trajectory of a particle departs from the streamline airflow due to a variety of physical forces. Mechanisms of particle deposition in the respiratory tract include: 1) inertial impaction; 2) gravitational settling; 3) Brownian motion; 4) interception; 5) electrostatic forces. These mechanisms will be briefed below. It should be noted that the deposition occurs when several mechanisms act simultaneously. A brief discussion is shown below. Discussion of all the deposition mechanisms has been included in several books in details.[25, 29, 30] 


\subsubsection{Deposition by inertial impaction}

Deposition by inertial impaction is the main mechanism for larger particles $(>5 \mu \mathrm{m})$ with high velocity. This mechanism occurs at the curvature when the airflow is deflected. While smaller and slower particles can move along the streamline, the larger and faster particles are unable to follow the streamline due to inertia. A parameter that determines the deposition efficiency is the Stokes number:[25, 29]

$$
S t k=\frac{\tau V}{D / 2}=\frac{\rho_{p} d_{p}^{2} V C_{c}}{9 \eta D} \quad(\text { Eq. 1-4) }
$$

where $\tau$ is the relaxation time, $d_{p}$ and $V$ are the particle diameter and velocity, respectively; $C_{c}$ is the Cunningham correction factor.

\subsubsection{Deposition by sedimentation}

Gravitational settling (sedimentation) is governed by Stokes's law (Eq. 1-1). Particle diameters between $0.5-3 \mu \mathrm{m}$ are deposited in the bronchial and alveolar regions by this mechanism. Both inertial impaction and gravitational settling are dependent on the relaxation time $(\tau)$ of the particle, which is defined as:[25]

$$
\tau=\frac{d_{p}^{2} \rho_{p}}{18 \eta} \quad(\text { Eq. 1-5) }
$$

Increased gravitational settling occurs by either breathholding or slow tidal breathing.[29]

\subsubsection{Deposition by Brownian motion}


Brownian motion (diffusion) dominates the deposition of particles smaller than $0.1 \mu \mathrm{m}$. The rate of diffusion, according to the Stokes-Einstein equation, is proportional to the temperature and inversely proportional to the particle size and air viscosity.

\subsubsection{Deposition by interception}

Interception is mainly relevant to particles with high aspect ratio (elongated). While flowing in the airway, elongated particles are predominantly aligned with streamlines with their long axis. Therefore, they have aerodynamic diameters $\left(d_{a}\right)$ that are predominantly related to their short axis (cross-sectional diameter). It is well known that elongated particles such as asbestos and mineral fibers are able to deposit in deep lung (alveolar region) because of their small aerodynamic diameters and the mechanism of interception. Once deposited, they have less chance of being cleared by macrophage due to their length.[25] Because of these characteristics, the use of elongated particles for drug delivery has attracted much attention. There are quite a few reports that elongated drug [31-34] or carrier [35, 36] particles enhance aerosolization performance exemplified as increase in fine particle fraction. However, the enhanced performance relies on the loose agglomeration of the fiber to facilitate fluidization and deaggregation during actuation. The performance could be poor when the fibrous particles are in contact along their length.[28] Powder flow is adversely influenced by increasing elongation ratio, which may also cause processing problems such as reduced content uniformity.

\subsubsection{Deposition by other mechanisms}

Besides the above mentioned mechanisms of respiratory deposition, several additional mechanisms may come into play. The electrostatic deposition may occur due to surface 
charges present on particles and the airway surfaces. Hygroscopic particle growth at elevated relative humidity and temperature in the respiratory tract may increase or decrease deposition velocity.[30]

It should be noted that deposited particles do not reside permanently in the respiratory systems, but are removed by respiratory clearance. The particles (i.e. drugs) are solubilized and taken up into blood circulation if they are soluble in bodily fluid. Otherwise, they are cleared by alveolar macrophage or mucociliary escalation (the upward movement of mucus due to coordinated cilia beating.

In order to enter the respiratory tract particles must be generated as aerosols. One of the major categories of aerosol delivery systems, dry powder inhaler, briefly mentioned earlier is described in detail below.

\subsection{Dry Powder Inhaler (DPI)}

The first commercial DPI was the Spinhaler ${ }^{\circledR}$ for the delivery of disodium cromoglycate to treat asthma.[37] Unlike pMDIs, passive DPIs actuated by the inspiratory flow of the patient require little inhalation coordination. In addition, the propellant-free dosage forms offer advantages of formulation stability and low environment impact. DPIs have become an important alternative to pMDIs for pulmonary drug delivery.[38-43] In the U.S., several successful DPI products, such as Turbuhaler ${ }^{\circledR}$ and Diskus ${ }^{\circledR}$, were launched in the market in the late 1980s and 1990s, respectively.[38] Currently, there are more than twenty passive (breath actuated) DPIs commercially available.[6, 43] As the advances of powder technology and device design occurred, next generation passive and active DPIs are expected to be 
approved by Food and Drug Administration. It has been predicted that they may supersede pMDIs as the most popular devices.[44, 45] The evolution of DPI is shown is shown in

\section{Appendix 1-A.}

All DPIs contain three basic features: device, formulation, and dose-metering system. For passive DPIs, the inspiratory flow is the only source of energy for particle resuspension from static powder bed. The device is designed to allow efficient resuspension through distinct fluidization and deaggregation mechanisms.[46] The formulation contains either respirable drug particles alone (having the aerodynamic diameter about 1-5 $\mu \mathrm{m}$ depending on the target site), or respirable drug with a carrier powder, usually $\alpha$-lactose monohydrate.[47] The carrier (typically $>30 \mu \mathrm{m}$ [48]) functions primarily to increase powder flow and facilitate drug aerosol formation, and act as a bulking agent for uniform capsule filling. There are mainly three types of metering systems including the pre-metered individual capsules, multiple dose blisters or strips, or dose reservoirs.[42] The device, formulation, and patient's inspiratory maneuver, determine the efficiency and reproducibility of delivery of therapeutic aerosols and, consequently, the probability of their reaching the desired site of action.[41, 4951] The next generation DPIs under development includes extra mechanisms that facilitate drug aerosolization. They include, but are not limited to impellors (Spiros), compressed air assist (Nektar), vibration (Oriel, Microdose), and impact hammers (3M, DelSys).[19] Novel dry powder inhaler (DPI) technology and particle engineering has yet to lead to the fundamental understanding required for predictable and reproducible pulmonary delivery of therapeutic aerosol in an airflow independent manner. 


\section{$\underline{\text { 1.3.1 Aerosol Formulation }}$}

The aerosol formulation consists of interactive physical mixtures of particles (drugs, carriers, and surface fines) maintained by thermodynamically favorable interfacial interactions at the solid-solid interface. The interparticulate interactions are a combination of van der Waals forces, capillary forces, electrostatic forces, and mechanical interlocking.[46] The aerosol formation requires the static powder to overcome these interparticulate interactions and resuspend the drug particle in an air stream. Besides the adhesion and cohesion, the interparticulate friction may also play an important role at the initiation of powder aerosolization.[52] Surface analytical approaches have demonstrated the importance of the interparticulate forces and formulation performance. The formulation performance may be considered in four transition states designated as dilation, flow, fluidization and deaggregation, which are most easily contemplated as sequential steps but from a practical standpoint are likely to be, more or less, instantaneous phenomena (Figure 1-2C). [46]

\subsubsection{Device}

\subsubsection{DPI device}

DPI device development,[6, 38, 40, 53-55] innovation,[43] mechanism of particle separation,[6] and the influence of inhaler selection [50] have been reviewed extensively. Each commercial DPI device has distinct dimensions and internal geometries. Thus, each has its distinct airflow parameters (e.g. specific resistance, $R_{D}$ ) and combination of aerosol generation mechanisms to enhance drug resuspension. The mechanisms of DPI fluidization include shear, capillary, and mechanical forces; and the mechanisms of particle 
deaggregation include relative motion, turbulence, shear stress and collision [46]. These mechanisms occur to a different extent in each inhaler device, but their relative importance in the device is not well understood yet.[6,46] The airflow pathways of commercial DPIs are usually short, tortuous, and sometimes include grids or baffles to maximize delivery efficiency, so their airflow conditions are usually not fully developed.[46] The device design is usually tailored to achieve maximum drug aerosolization efficiency of a specific powder formulation. The combination of the formulation and device is considered the "drug product". Therefore, once a product has been developed the efficiency and reproducibility of aerosol delivery by a DPI device-drug formulation combination are measured as the component characteristics give rise to a single performance outcome. However, during preliminary studies intended to focus on formulation effects alone to aid in optimization, the effect of device, required to generate the aerosol, is often underestimated or simply ignored. Due to the large variation caused by incompletely developed airflow condition and diverse dispersion mechanisms upon inhalation, the evaluation of the formulation performance effect is often inconsistent and confounded by the device effect. The use of different devices by different researchers prevents the comparison of performance results even if the same airflow rate, or pressure drop, is applied. It would be desirable to assess and understand the elements of the formulation that contribute to improved performance independently of the unique inhaler design. Therefore, it is desirable to adopt standardized devices that will allow comparison of formulations between laboratories independently of specific inhalers.

\subsubsection{Standardized entrainment tubes (SETs)}

SETs were designed and made in order to respond to the needs for standardized devices to evaluate formulations.[56] These SETs were made of polished stainless steel and designed as 
device-independent tools that encompass a wide range of pressure drops used in commercial DPIs at defined airflow rate.[56] The geometry and dimension of the SETs are shown in Table 1-1. The principal aerosol generation mechanisms of the SETs are turbulent shear force fluidization and deaggregation [56]. Unique features, often found in DPIs, such as grids, tortuous channels and other forms of baffles, are not present in the SETs, as they are characteristic device design elements matched to particular formulations intended for specific drug products and do not easily lend themselves to standardization. Unlike the commercial DPI devices, the SETs have fully developed (Poiseuille's law in a flow field through a cylindrical pipe) and well-characterized airflow parameters designated as shear stress $\left(\tau_{s}\right)$, Reynold's number $(R e)$, pressure drop $(\Delta P)$ and power [56], which allow focus on the formulation effect. Among these parameters, the viscous turbulent stress is directly related to the fluid energy dissipation rate, which is influenced by aerodynamic acceleration, shear flow as well as by impaction or other mechanical forces.[57] This parameter varies within the flow cross-section and also depends on the flow geometry and Reynolds number.[57] In addition to turbulent shear forces, the impaction and momentum transfer are perhaps the most important mechanisms for deaggregation because they are volume (or weight) related.[58, 59] However, it is reasonable to suggest that particles which can be separated by shear forces may more readily be removed under the influence of impaction and momentum transfer.[60]

\subsection{Fundamental Intermolecular and Surface Forces}

\subsubsection{Adhesion and Cohesion Forces}

The intermolecular forces are essentially electrostatic in origin and their rigorous treatment 
requires consideration of quantum electrodynamics in which the spatial distribution of the electron clouds is described by Schrödinger equation.[61] For practical operation, these forces are roughly classified into commonly encountered forms including: (1) van der Waals forces which are attributed to polarization forces that arise from the dipole moments induced in atoms or molecules; (2) hydrogen bonds which are a special dipole-dipole interaction of polarized hydrogen atom with an electronegative atom; (3) chemical bonding including covalent bonds and ionic bonds at shorter range; (4) Coulomb force arising between charged molecules which are purely electrostatic in nature, although all of these forces have the same fundamental origin.[62] In the pharmaceutical sciences, these attractive physical forces are manifested in different forms such as surface adsorption, protein binding, hydrophobic interaction, solvation, crystallization, self-assembly, due to the difference in molecular properties at different force range. These forces are often discussed in connection with intermolecular surface physics and chemistry. [63, 64]

In principle, the fundamental physical forces involved in macrobodies such as colloids, microparticles and surfaces, namely the interparticulate or surface forces, are similar to those at the molecular level.[62, 65] These attractive physical forces in dry powder aerosol formulations are mainly classified as van der Waals, electrostatic, and capillary forces. Other forces including frictional forces, mechanical interlocking, and hydrogen bonding are not frequently studied, yet still important.[66] They are the manifestation of combining relations of the above described intermolecular forces acting between larger particles or extended surfaces and should be operative between both molecules and particles.[67] They lead to particle aggregation (adhesion/cohesion).[68] Among them, van der Waals forces are typically dominant in uncharged dry powders.[69] The magnitude (Figure 1-3) of the van der 
Waals, electrostatic, and capillary forces between ideal binary system (ideal assumes to be atomically flat, surface homogeneous, and rigid bodies) was estimated by calculation previously.[58, 66] The van der Waals forces are approximately two orders of magnitude greater than electrostatic forces and capillary forces. The latter arising from capillary condensation will prevail only at high relative humidity. The majority of publications show calculations of the adhesion/cohesion forces in uncharged dry powders based only on the contribution of van der Waals forces. Through interaction potential of the form $w(r)=-C / h^{6}$ (Lennard-Jones attractive potential, where $C$ is the coefficient of intermolecular pair potential, $h$ is the separation distance) and non-retardation assumption, the van der Waals forces can be semi-quantitatively described as below:[62, $65,67,70]$

$$
\begin{gathered}
F_{v d w}=\frac{A R}{6 h^{2}} \frac{R_{1} R_{2}}{R_{1}+R_{2}}=\frac{A R}{12 h^{2}} \quad\left(R_{1}=R_{2}=R, \text { for two identical spheres }\right) \\
F_{v d w}=\frac{A R}{6 h^{2}} \quad\left(R_{2}>R_{1}, \text { for sphere and surface }\right) \quad \text { (Eq. 1-7) }
\end{gathered}
$$

where $A$ is the Hamaker constant (typically $\sim 10^{-19} \mathrm{~J}$ ) depending on the molecular properties of the materials involved [62], $R$ is the particle radius (m), and $h$ is the separation distance (m). Eqs. 1-6 and 1-7 are useful for understanding the origin and magnitude of adhesion/cohesion forces as they are proportional to the particle size. Because gravitational forces diminish quicker than van der Waals force when particle size is small, the magnitude of van der Waals forces become appreciable and the dry powder becomes adhesive/cohesive, when the particle sizes are less than $10 \mu \mathrm{m} .[69]$

The description of interparticulate forces can also be achieved by adopting a thermodynamic approach because the work of adhesion/cohesion is closely related to surface 
energetics. The theoretical treatment that correlates the interparticulate forces between two rigid spheres and thermodynamic work of adhesion is known as Derjaguin approximation (valid when the range of interaction is much less than the local radii of particle curvature) (Eq. 1-8).[71]

$$
F_{a d}=2 \pi\left(\frac{R_{1} R_{2}}{R_{1}+R_{2}}\right) W_{a b}=2 \pi\left(\frac{R_{1} R_{2}}{R_{1}+R_{2}}\right)\left(\gamma_{a}+\gamma_{b}-\gamma_{a b}\right)
$$

where $F_{a d}$ is the adhesion force $(\mathrm{N}) ; W_{a b}$ is the work of adhesion between two particles $(\mathrm{N} / \mathrm{m})$; $\gamma_{a}, \gamma_{b}$, and $\gamma_{a b}$ are the surface free energy of substance $a, b$, and interfacial energy between $a$ and $b\left(\mathrm{~J} / \mathrm{m}^{2}\right)$.

Although the quantification of van der Waals forces between ideal particles appears to be straightforward, the measured forces are not directly translatable to aerosol performance. This may be attributed to the complexity of real particles, which are inherently heterogeneous. Van der Waals forces between actual pharmaceutical particles require consideration of heterogeneity which can be roughly classified from geometric and energetic perspectives. The former includes particle size distribution (including the presence of a ternary component), morphology, surface asperities, meso- or micro-pores, and particle deformation, and the latter, the energy density difference caused by surface imperfection such as amorphous content, polymorphic state, or surface impurities. Even perfectly crystalline materials have different energy distributions due to their molecular orientation and crystal habit. The form of distribution varies with different dry powder material. They will influence the interparticulate forces, heat capacity, mechanical stress, etc. The practical approach to describing energy heterogeneity is to average local thermodynamic quantities 
and treat them statistically (i.e. distribution function).[72] Several distribution functions have been used in the literature, such as uniform distribution, normal distribution, log-normal distribution, exponential distribution, and Rayleigh distribution. They are generally discussed in the literature of surface adsorption or surface chemistry.[67, 72] Moreover, the surface heterogeneity feature is a characteristic of mutual interaction between particles rather than an independent particle.

Van der Waals forces decay rapidly with increasing separation distance and are effective only within a separation distance less than $100 \mathrm{~nm}$ [67], so they are quite sensitive to particle shape and surface roughness change.[73] Eqs 1-6 and 1-7 for van der Waals forces prediction are often poor for pharmaceutical powders because the particle surfaces are normally covered by asperities. Roughness arises from numerous small steps, kinks, defects or flat regions. They can be evaluated mathematically in terms of irregular oscillations around a mean value.[74] Different models such as Fourier, fractal, and chaos analysis have been used.[74] The change of surface roughness can change van der Waals forces by several orders of magnitude. The magnitude of the interparticulate forces is also affected by the number of mutual points of contact (the coordination number).[66] The adhesion/cohesion can be overestimated or underestimated based on the effective contact area caused by surface roughness.[27]

Electrostatic forces can be attractive or repulsive. They occur by means of contact charging, coulombic interaction, and induced charging. Contact charging occurs during contact between uncharged and unlike particles by electron transfer until equilibrium state of the Fermi level is achieved. The magnitude of the contact potential force $\left(F_{c}\right)$ may be expressed as:[27] 


$$
F_{c}=\frac{2 \pi q^{2}}{A} \quad(\text { Eq. } 1-9)
$$

where $q$ is the particle charge on detachment from a substrate; $A$ is the contact area between the particle and the substrate.

Coulombic forces occur when two charged particles are brought together. Either attraction or repulsion is possible depending on the electrical charge signs. The magnitude of Coulombic forces may be expressed as:[75]

$$
F_{q}=\frac{q_{1} q_{2}}{4 \pi \varepsilon h^{2}} \quad(\text { Eq. } 1-10)
$$

where $q_{1}$ and $q_{2}$ are the electrical charges on the two particles, respectively; $\varepsilon$ is the permittivity and $h$ is the separation distance.

The induced charging occurs when a charged particle, $q$, approaches an uncharged particle of radius $R$. The magnitude of induced charging forces may be calculated as:[75]

$$
F_{e}=q^{2}\left[1-\frac{h}{\left(R^{2}+h^{2}\right)^{0.5}}\right] \times \frac{1}{16 \pi \varepsilon_{0} h^{2}}
$$

where $h$ is the separation distance; $\varepsilon_{0}$ is the permittivity.

Capillary forces rise between particles following capillary condensation of water vapor at high relative humidity $(\mathrm{RH})$. The Laplace pressure develops due to the water meniscus formed in the contact zone pulls particles together. The capillary forces between two spherical particles may be described as [62]:

$$
F_{c}=4 \pi R * \gamma_{L} \cos \theta+4 \pi R * \gamma_{S L}
$$


where $F_{c}$ is the capillary force; $R^{*}$ is the harmonic mean of the particle radii; $\gamma_{L}$ is the surface tension of water $\left(72.8 \mathrm{mN} / \mathrm{m}\right.$ at $\left.20^{\circ} \mathrm{C}\right) ; \gamma_{S L}$ is the solid-liquid interfacial energy; $\theta$ is the measured contact angle that water is supposed to form with the particle surface.

Real particles will deform (change in shape and asperities) because of the attractive interparticulate forces, resulting in a finite contact area even without external force load.[62] The physicochemical and viscoelastic properties of the particles will influence the deformation and effective contact area. The determination of the pull-off force between deformable bodies has been subjected to several theoretical treatments. Pharmaceutical powder aerosols are in general described by adhesive elastic contact using the JohnsonKendall-Roberts (JKR)(Eq. 1-13) [76] or the Derjaguin-Muller-Toporov (DMT)(Eq. 1-14) [77] models.

$$
\begin{gathered}
F_{a d}=\frac{3}{2} \pi R^{*} W_{a d} \\
F_{a d}=2 \pi R^{*} W_{a d}
\end{gathered}
$$

where $F_{a d}$ is the adhesion force $(\mathrm{N}), R^{*}$ is the harmonic mean of the particle radii $(\mathrm{m}), W_{a d}$ is the work of adhesion $(\mathrm{N} / \mathrm{m})$. The choice of the model depends on the interacting materials and the particle geometries. The DMT model is applicable for hard material with low surface energies and high elastic moduli (Young's modulus $>10^{9} \mathrm{~N} / \mathrm{m}^{2}$ ), and considers attractive force beyond the contact region. The JKR model is for softer material and neglects the attractive force outside the contact circle, which indicates the existence of a neck of finite height.[27] The transition of DMT to JKR model can be interpreted by a dimensionless parameter known as Tabor parameter.[78] 


\subsubsection{Deaggregation (Separation) Forces}

Two-phase systems, the solid particles and air molecules with which they interact, play important roles in powder dynamics. As mentioned above, the whole aerosolization can be roughly categorized into four phases starting from static powder bed, dilation, fluidization, to drug resuspension.[79] When the airflow entrains through a DPI, it transfers the kinetic energy of the continuous bombardment of air molecules into the powder bed for powder entrainment and deaggregation. According to Rumpf's theory, the particle separation occurs when the adhesion forces are supplanted by the applied removal force; whereas Kendall's theory state that the separation occurs when the energy consumption is proportional to the formation of new surfaces.[80] For carrier-based DPIs, the separation forces include 1) drag and lift, 2) shear and friction, and 3) inertial forces. The aerodynamic drag and shear displacement are proportional to the particle cross-sectional area $\left(F_{\text {drag }} \propto d^{2}\right.$; area related) (Eq. 1-15).[66] It is increasingly difficult to fluidize smaller particles because adhesive/cohesive forces are generally dependent on their diameter, $d$. Whereas the inertial forces are related to kinetic energy function such as collision, rotation, and vibration $\left(E_{k} \propto d^{3}\right.$; volume related) (Eq. 1-16),[66] where $C_{d}$ is the drag coefficient; $d$ is the diameter of the particle (m); $V$ is the airflow velocity $(\mathrm{m} / \mathrm{s}) ; \rho_{a}$ is the density of the air $\left(\mathrm{kg} / \mathrm{m}^{3}\right)$; and $\rho_{p}$ is the density of the particle $\left(\mathrm{kg} / \mathrm{m}^{3}\right)$. Shear and friction forces between drugs and carriers are also proportional to $d^{3}$.[58] A more detailed equation that describes the components of predominating forces affecting the relative motion of a particle can be found in the literature.[66]

$$
F_{\text {drag }}=C_{d} \frac{\pi}{8} \rho_{a} d^{2} V^{2}
$$




$$
E_{k}=\frac{\pi}{12} \rho_{p} d^{3} V^{2} \quad(\text { Eq. } 1-16)
$$

The airflow in the DPI device may be laminar, transitional, or turbulent, which can be described by Reynolds number of the device $\left(R e_{d}\right)$.[66] The majority of DPIs are designed to have turbulent airflow to facilitate particle deaggregation $\left(\operatorname{Re}_{d}>4000\right.$ for fully developed airflow in cylindrical tube), or a turbulent core surrounded by a laminar envelope.[66] Besides $R e_{d}$, factors affecting particle entrainment and deaggregation in an air stream include shear stress $\left(\tau_{s}, \mathrm{~N} / \mathrm{m}^{2}\right)$, pressure drop $\left(\Delta P, \mathrm{~N} / \mathrm{m}^{2}\right)$, and power $(\mathrm{Nm} / \mathrm{s})$. [56] The volume airflow rate required to produce particle deaggregation force to overcome van der Waals forces was estimated previously and can be expressed as $Q>110 d_{p}^{-4 / 7}(\mathrm{~L} / \mathrm{min}), d_{p}$ is the diameter of the particle $(\mu \mathrm{m}) \cdot[81]$

The drug aerosolization efficiency of a dry powder aerosol formulation is generally characterized by in vitro performance evaluation, typically an inertial method is employed such as liquid impinger sampling or Andersen cascade impaction.[46]

\subsection{Aerosolization Performance}

\subsubsection{Definition of terms}

With respect to dry powder aerosol performance efficiency and reproducibility, the terms emitted dose, fine particle mass or fraction, and mass median aerodynamic diameter are frequently used. The emitted dose (ED) represents the proportion of the nominal dose delivered, the rest remaining in the device. The fine particle mass (FPM) or fraction (FPF) is the mass, or proportion with respect to the nominal dose in a size range considered sufficiently small to enter the lungs. The numerical cut-off size defining the FPM or FPF is 
dictated by the sampling airflow rate and the calibrated cutoff of the inertial sampling device with which aerodynamic size is measured. It is frequently the case that the FPM or FPF are defined with respect to proportion of mass or faction of the total in particles below an aerodynamic diameter of approximately $5 \mu \mathrm{m}$ (dependent on the sampler and operating conditions). The mass median aerodynamic diameter (MMAD) represents the aerodynamic particle size for which $50 \%$ of the total aerosol by mass has smaller aerodynamic diameters. Both FPM (or FPF) and MMAD represents the degree of deaggregation, where larger FPM and smaller MMAD values represent greater deaggregation.[56]

\subsubsection{Aerosolization performance}

The aerosolization performance of DPI systems depends on different perspectives mentioned above. The effect of lactose monohydrate carrier on the aerosolization has been explored extensively.[49, 82] An overview of strategies involving lactose monohydrate modification to change drug-carrier interaction and improve aerosolization performance is shown in Table 1-2.[83] Increased drug aerosolization was often observed with smaller carrier size, because the proper size of carrier particles may effectively disrupt the drug self-agglomerates.[60, 84] However, it was also reported that increasing the carrier size had little influence [52] or increase FPF [85] in some formulations. The addition of fine excipients $(<10 \mu \mathrm{m})$ generally increases FPF, and an optimum concentration of fines exist.[86, 87] The carrier shape affects the formulation performance, but both increased [35] and decreased FPF [36] were reported when the elongation ratio of lactose monohydrate increased. The increased FPF could possibly be explained as the increasing aerodynamic drag forces to the elongated carriers, whereas the decreased performance in another system was possibly affected by poorer 
powder flow due to poorer blend content uniformity and irreproducible ED. The influence of polymorphism could confound the performance results caused by elongation ratio. The surface roughness could influence powder performance in two ways. Lower FPF was obtained from carriers with macroscopic surface roughness or smooth surfaces, whereas higher FPF was obtained from carriers with microscopic surface roughness.[88, 89] The key is the effective drug-carrier contact areas which determine the magnitude of adhesion. Particle surface coating with force control agents (e.g. leucine, magnesium stearate) could effectively reduce the cohesive-adhesive ratio (characterized by AFM cohesive-adhesive balance approach) and improve aerosolization performance.[90] Surface modification by etching of carrier lactose monohydrate improved performance, due to the change in surface roughness and reduced drug-carrier adhesion.[91, 92] The particle engineering techniques $[93,94]$ provide versatile methods to change particle size, morphology, surface roughness, envelope density, crystallinity, and surface electrostatic charge, all of which may have significant impact on the aerosolization performance efficiency.

The goals of these aerosol performance studies are straightforward. They include: 1) to improve the aerosolization performance efficiency by maximizing the FPF (or FPM, defined in Section 1.5.1 above); 2) to develop device independent formulation (i.e. the same FPF across different aerodynamic shear) and; 3) to achieve formulation prediction. However, in spite of extensive research in manipulation of interparticulate interactions by above mentioned methods, the list of parameters analyzed and the performance properties measured indicates the unfortunate nature of research in this field, since it is not evident that the phenomenology that naturally precedes the framing of hypotheses to facilitate an understanding of underlying scientific principles has evolved beyond its empirical origins. 
New regulatory initiatives in Quality by Design will facilitate concordance of experimental design, and development of standardized materials and methods supporting progress in this field.

\subsubsection{Current trend for performance prediction of aerosol formulation}

The relationship between the physicochemical properties of dry powder aerosols and the aerodynamic aerosolization performance efficiency could be rather complex because a myriad of influencing factors described above. Real pharmaceutical fine powders are affected by both their intrinsic characteristics and environmental factors. They are susceptible to change over time. Each change will result in a formulation with new physicochemical properties that may influence performance.[95] Unique inhaler devices and aerosolization conditions further confound the aerosol performance evaluation and limit the potential to predict behavior. The aerosolization performance prediction remains a challenge due to the limited fundamental understanding of interparticulate interactions in heterogeneous condition and how they can be related to the deaggregation processes even in the simplest dry powder

aerosol system. It is imperative to extend qualitative observations to obtain quantitative reproducible measurements. Currently, the performance prediction can be roughly classified into two categories: The correlation of performance data with 1) formulation properties and 2) airflow properties.

Performance prediction of a DPI formulation is, to a large extent, achieved by correlation of performance data (such as $E D, F P F, M M A D$ ) with formulation properties. The performance is generally evaluated at fixed airflow condition (e.g. fixed airflow rate, 
pressure drop, etc). A straightforward approach frequently used is the correlation of performance with interparticulate interactions. Traditional techniques for interparticulate force measurement include particle detachment following centrifugation, vibration, impact separation, and the rapidly developed atomic force microscopy (AFM) technique.[96] The AFM colloid probe and pull-off force techniques allow interparticulate force measurement at single particle level. It is well known that geometric and energetic heterogeneity often cause log-normal distribution of the pull-off forces.[97] However, practically, it has been observed that dry powder formulations having similar adhesion/cohesion do not necessarily result in similar aerodynamic behavior of the drug when lactose monohydrate based formulations were studied.[52, 98] This deviation may be attributed to different sites of adhesion.[52] Moreover, increased cohesion was correlated with decreased $F P F[99,100]$ or increased $F P F$ [101] when using drug-only formulation. These paradoxical correlation results can possibly be explained by variation in the fluidization and the effectiveness of aerodynamic deaggregation exerted on the agglomerates.[101] It is clear that the pull-off force alone may not be a reliable parameter for performance prediction. There are reports of the influence of the contribution of individual interparticulate forces (van der Waals, capillary, electrostatic forces) on performance. However, the correlation of formulation performance with individual component of these fundamental forces was rare.

In recent years, for the purpose of performance efficiency optimization, indirect correlations of performance data with particle size, $[102-104]$ carrier type, $[105,106]$ particle morphology,[107] surface roughness,[92, 100, 108] surface energy,[109-112] ternary component, $[104,113,114]$ relative humidity $(\mathrm{RH} \%)[115,116]$ have been explored. These parameters have a great influence on the particulate interaction. Caution should still be 
exercised when applying some of these parameters. For example, the correlation of performance with the increasing surface roughness, was known to either increase $[35,108]$ or decrease $[92,100,117]$ the interparticulate forces depending on the true area of contact. Different surface analytical techniques have been applied to evaluate the change in correlation between particle parameters and performance. AFM is a powerful and versatile technique that is playing an important role in surface characterization of various components related to the fundamental forces and geometrical features that contribute to adhesion/cohesion under a variety of environmental conditions.[118] This technique has been used extensively for formulation screening and performance prediction. It is worth mentioning that performance efficiency of micronized drug particles is dependent on the drugs' cohesive and adhesive properties and optimum performance is achieved when the ratio of cohesion to adhesion is balanced. An approach, designated the cohesive-adhesive balance $(\mathrm{CAB})$ method has been described $[119,120]$ and the aerosol performance was correlated with the ratio of cohesive and adhesive forces.[101, 105] Although factors such as surface roughness can significantly influence the performance, this method avoids the need for measurement of the area of contact, by a normalization technique. Overall this method has been useful for formulation screening [121] and correlation with the results of aerosolization performance mechanism studies in the more sophisticated ternary blending system.[113] A slightly cohesive drug-carrier $\mathrm{CAB}$ ratio was suggested for optimum formulation performance.[121] Although rapid development of the AFM technique has occurred, it should be noted that the correlation using one technique may not always be reliable. AFM substrates are usually prepared by recrystallization or high-pressure compaction, which may not be a true representation of a relevant pharmaceutical formulation. 
Other surface analytical techniques such as inverse gas chromatography (IGC) and contact angle measurement can provide complementary data for surface energy analysis.

Other correlations have been explored for performance data with respect to alternative aerosol powder properties such as milling effect,[104] Hildebrand solubility parameter,[122] drug-carrier ratio,[123] Carr's flowability index,[124] elongation ratio,[35] avalanche time,[98] and the amount of particle surface coating.[125, 126] These parameters are more or less indirectly related to the fundamental interparticulate forces or surface energetics.

Conversely, the correlation of performance with the airflow properties (e.g. shear stress) is much less frequently reported than the relationship to formulation properties. This may be attributed to: 1) the requirement for a performance study to control a variety of influencing factors, and the airflow is the easiest to fix once the inhaler devices and airflow rate are determined; 2) The performance efficiency among different formulations is usually compared qualitatively and categorically (i.e. rank order comparison); 3) Many of the studies of aerodynamic behavior under various air flow conditions focus on the inhaler device development. (For example, the computation fluid dynamics (CFD) aided inhaler device design); 4) The formulation effect is often confounded by the inhaler device effect. More than twenty inhalers $[43,66]$ are currently marketed with a variety of mechanisms of drug aerosolization, the results and findings in one system cannot be extrapolated directly to another system. Nevertheless, the formulation performance as a function of airflow parameters is very important because the optimum formulation cannot be determined unless its performance under the airflow conditions within a relevant inspiratory range is evaluated. For example, the optimized formulation used in Inhalator $^{\circledR}$ (high shear stress) may not 
necessarily disperse efficiently in Rotahaler $^{\circledR}$ (low shear stress) at the same airflow rate. Moreover, most of the commercial DPIs require patients' inspiration for pulmonary drug delivery. The airflow parameters are dependent on the airflow rate (patients' maneuver) which may vary greatly. Performance reproducibility in a wide respirable range is a key to the successful formulation development and FDA approval for pulmonary delivery. Currently, the conventional formulation optimization and reproducibility study remains an empirical estimation based on iteration by trial and error.

Performance efficiency is directly related to specific resistance $\left(R_{D}\right)$, which is dependent on the geometry and dimension of the inhaler devices.[127] At given airflow rate, inhaler devices with higher $R_{D}$ are expected to generate greater turbulence and result in higher $F P F$.[128] However, higher $R_{D}$ will result in lower volumetric airflow rate due to the capability of patients' maneuver. Powder flow is correlated directly with ease of particle separation and aerosol performance at given $R_{D}$ (same inhaler device).[129] This indicates that increasing inspiratory effort corresponds to increase in FPF. More recently, Chan et al [130] studied the influence of airflow at 8 different airflow rates on the performance using mannitol powder. They observed that an increase in " $F P F_{\text {Impinger" }}$ " $\left(F P F_{\text {Impinger }}\right.$ represents $F P F$ that separate from the influence of capsule, device and throat retention) occurred at lower airflow rate between 30 and $75 \mathrm{~L} / \mathrm{min}$ but reached a plateau at higher airflow rate. They also applied a CFD approach to track the fate of $1000-5000$ particles through the airstream generated inside the Aerolizer ${ }^{\circledR}$, and concluded that device flow field generated from simpler geometry is necessary for deaggregation mechanism studies such as turbulence and impaction levels.[130, 131] It should be noted that the airflow rate based on a patient's inhalation covers a very narrow range of inspiratory capacity, while $R_{D}$ tolerates a wider 
range which can be achieved by device innovation.[128] Recently, a CFD approach was used to model airflow path and particle trajectories using $\sim 1000$ particles. The particles were assumed to deaggregate when the fluid based torque exceeded the separation torque.[59] Both fluid-based and impact-based effects were evaluated.[59] Due to the complexity of the deaggregation process, the CFD approach is only capable of describing a small population of particles but not the entire pharmaceutical powder. In 2006, de Boer et al [132] evaluated the relationship of $F P F$ and pressure drop $(\triangle P)$ using four commercial inhaler devices with distinct mechanisms of deaggregation upon which the performance was highly dependent. The complexity of deaggregation makes it difficult to separate the effects of formulation heterogeneity and the confounding airflow conditions other than $\Delta P$. A global nonideality of the experimental system was observed instead of attempting to deconvolute the confounded separate effects.[132] Also in 2006, Louey et al [56] described standardized entrainment tubes (SETs). These SETs were characterized using four airflow parameters including pressure drop $(\Delta P)$, power, Reynold's number $(R e)$, and shear stress $\left(\tau_{s}\right)$ at airflow rates of 28.3 and $60 \mathrm{~L} / \mathrm{min}$. An increase in these parameters across the device at the same airflow rate corresponds to increased drug deaggregation. The airflow conditions of these SETs encompass those of commercial inhalers, and the application of well-defined SETs for performance studies eliminates the confounding factors caused by inhaler devices and allows focus on formulation effect. A semi-quantitative correlation of performance data including fine particle mass $(F P M)$ and mass median aerodynamic diameter $(M M A D)$ with these airflow parameters was then evaluated. For Ventolin ${ }^{\mathrm{TM}}$ rotacaps (a lactose based albuterol sulfate formulation), hyperbolic relationship and exponential decay were suggested for FPMairflow parameters and $M M A D$-airflow parameters correlation, respectively.[56] Yet, no 
regression analyses were performed to further quantify these relationships due to the limited quantity of initial data.

\subsection{Problem Statement: Delivery Efficiency and Reproducibility}

Despite the emergence of novel particle engineering and device technology, efficient and reproducible pulmonary delivery of dry powder aerosols in an airflow independent response to shear stress still represent a great challenge. This is partly due to a lack of fundamental understanding of the behavior of drug microparticles in heterogeneous environments arising from a variety of factors (e.g. surface heterogeneity of adsorption sites [133]). Analytical approaches such as AFM cohesive-adhesive balance have demonstrated the importance of the interplay of the particulate forces and deaggregation. However, a direct correlation of pharmaceutical particulate interaction (microscopic) with the aerosol performance (macroscopic) data is not readily available, because of the heterogeous nature of both drug and carrier surfaces, and multiple assumptions (e.g. Derjaguin approximation) for direct interparticulate interaction measurement. The formulation design and optimization are often confounded by non-standardized devices, the heterogeneous nature of drug and carrier surfaces, and assumptions with respect to force (or surface energy) measurement. Performance prediction remains an empirical estimation based on iteration by trial and error. In addition to the collecting and analyzing existing results from the literature, we should evolve methods that convey the fundamental interparticulate interactions and applied such ideas for the formulation evaluation and prediction. Understanding of the fundamental adhesion/cohesion and heterogeneous particle deaggregation is still the key for the scientific development of DPIs. 


\subsection{Hypothesis and Objectives}

The phase-out of ozone depleting propellants from pharmaceutical aerosol products has created a demand for alternative methods of delivering locally acting drugs for the treatment of asthma and COPD. Dry powder aerosol formulations consist largely of blends of drug with lactose monohydrate. These formulations are frequently inefficient and irreproducible in their delivery of drugs. In order to improve performance prediction and optimization of DPI formulations, we will characterize the physicochemical properties of carrier-based DPI formulations at rest and investigate the heterogeneous nature of these formulations to the aerosolization performance using SETs. Towards these aims, and based on our knowledge that the fundamental forces that govern the particle-particle interactions are similar to intermolecular and surface interactions, we hypothesize that:

1) The application of SETs for formulation performance evaluation can minimize the confounding factors caused by inhaler devices and reveal the relationship of airflow $\underline{\text { conditions and carrier-based dry powder formulation performance }}$

2) The models of molecular surface association described by adsorption expressions can be adapted to fit shear displacement observations.

3) The carrier surface treatment can reduce the surface heterogeneity and improve the performance efficiency of dry powder aerosol formulation.

The arrangement of the major objectives that address the hypotheses is shown below:

1) Discriminatory assessment of dry powder aerosol performance using SETs and carrierbased formulations. In Chapter 2, we started with the physicochemical characterization of lactose monohydrate batches and their interactive physical mixtures with micronized model 
drug albuterol sulfate. Then, in Chapter 3, we presented an important and comprehensive experimental investigation of formulations ( 4 drugs $\times 4$ lactose monohydrate carriers) which led to the discovery of a novel predictive correlation method, namely the powder aerosol deaggregation equation (PADE). The performance evaluation of sixteen lactose-based formulations using novel PADE method was analyzed using conventional method (by DesignExpert analysis) and compared with the PADE method.

2) Chapter 4 and 6 represented two recent trends for the next generation DPI formulation development that either alternative carriers (Chapter 4) or surface modification of existing lactose monohydrate (Chapter 6) were explored for formulation optimization. The robustness of the PADE method was examined and extended to these systems.

3) Mechanistic evaluation of performance data by PADE. A theoretical interpretation and comparison of surface physical phenomena including surface adsorption, protein binding, and powder deaggregation were demonstrated in Chapter 5. The predictive PADE regression analyses were exemplified by the evaluation of SETs vs. commercial DPIs and storage effect. It is assumed that the particle deaggregation has a statistical origin associated with turbulent flow character. The selection of adapted surface adsorption models as the possible link between macroscopic statistical thermodynamics and microscopic individual particle deaggregation was rationalized. Also in Chapter 5, extra SETs were characterized and aerosolization performance was evaluated when more accurate data fittings were explored that consider surface heterogeneity of dry powder systems. The adapted surface adsorption models were further compared by introducing the heterogeneity factors associated with the selected adsorption theories. Several applications of the PADE were included. 
4) The surface modification of lactose monohydrate (Chapter 6) also served as a probe for the study of PADE mechanism.

5) Finally, in Chapter 7, in addition to a summary of the newly developed theory, some guidance for future development of DPI formulation was discussed.

\subsection{Summary}

In this chapter, important definitions and a brief history of pharmaceutical inhalation therapy have been presented. A short discussion of the therapeutic implications in respiratory drug delivery, including the mechanisms of particle deposition in the respiratory tract, a chronic airway disease, asthma, and the mechanisms of drug action for treatment of this disease were outlined. Two major components of DPI, the dry powder formulation and the aerosolization device, were elaborated. The DPI devices were compared with the SETs from their mechanisms of fluidization and deaggregation. An obvious advantage of SETs that allowed focus on formulation optimization was highlighted. The fundamental interparticulate and surface forces that govern the static powder behavior, and the fundamental forces responsible for powder aerosolization were recapitulated. These interparticulate interactions served as the foundation of all the following dry powder formulation studies. A mini-review of the aerosolization performance studies and current trend for formulation prediction and optimization was then on introduced, which led to the problem statement about improving the aerosolization efficiency and reproducibility. Finally, the hypotheses and the objectives of each following chapters were stated. 
Table 1-1. SET Specifications (Dimensions and Airflow Parameters at $Q=60 \mathrm{~L} / \mathrm{min}$ ). [56]

\begin{tabular}{|c|c|c|c|c|c|c|c|}
\hline \multirow[b]{2}{*}{ SET } & \multicolumn{5}{|c|}{ Dimensions $(\mathrm{mm}) * *$} & \multicolumn{2}{|c|}{ Airflow parameters } \\
\hline & $\begin{array}{c}\text { External } \\
\text { outlet } \\
\text { length } \\
\text { (a) }\end{array}$ & $\begin{array}{c}\text { Inlet } \\
\text { length } \\
\text { (b) }\end{array}$ & $\begin{array}{l}\text { Internal } \\
\text { outlet } \\
\text { length } \\
\text { (c) }\end{array}$ & $\begin{array}{l}\text { Inlet outer } \\
\text { diameter } \\
\text { (e) }\end{array}$ & $\begin{array}{l}\text { Inlet inner } \\
\text { diameter } \\
\text { (f) }\end{array}$ & $\begin{array}{c}\text { Reynold's } \\
\text { No., Re, }\end{array}$ & $\begin{array}{c}\text { Shear Stress } \\
\left(\mathbf{N} / \mathbf{m}^{2}\right)\end{array}$ \\
\hline A & 150 & 300 & 140 & 6.3 & 4.5 & 18443 & 13.143 \\
\hline B & 135 & 300 & 125 & 7.9 & 6.2 & 13440 & 4.342 \\
\hline $\mathbf{C}$ & 127 & 600 & 117 & 9.5 & 7.7 & 11066 & 2.199 \\
\hline D & 100 & 600 & 90 & 12.7 & 11.0 & 7720 & 0.624 \\
\hline
\end{tabular}

* The SET-A and B use peripherally-located dosage table. \# The SET-C and D use centrally-located dosage table.

** The powder dosage length (d) is set at $10 \mathrm{~mm}$. 
Table 1-2. Overview of Strategies that Control Drug-Carrier Interactions for In Vitro Aerosolization Optimization

\begin{tabular}{|c|c|c|c|c|}
\hline \multicolumn{2}{|c|}{ Modification } & \multicolumn{2}{|r|}{ Major Influencing Factors } & \multirow{2}{*}{$\begin{array}{c}\text { References } \\
{[84],[103],[123]}\end{array}$} \\
\hline \multirow{15}{*}{\multicolumn{2}{|c|}{$\stackrel{\infty 00}{\Xi}$}} & \multirow{7}{*}{$\begin{array}{l}\text { Physicochemic } \\
\text { al properties }\end{array}$} & Dose $(\% \mathrm{w} / \mathrm{w}$ conc.), drug carrier ratio & \\
\hline & & & Drug properties: cohesiveness, adhesiveness & [101], [103], [134] \\
\hline & & & Size and size distribution, polydispersity & {$[135,136]$} \\
\hline & & & Morphology, polymorph & [31], [36], [107], [137] \\
\hline & & & Surface roughness & [138], [139], [109], [140-142] \\
\hline & & & Crystallinity, surface amorphous content & {$[102],[143]$} \\
\hline & & & Crystal habit & {$[144]$} \\
\hline & & \multirow[t]{2}{*}{ Surface coating } & $\begin{array}{c}\text { Force control agents: magnesium stearate, leucine, } \\
\text { lecithin, polymer }\end{array}$ & [90], [109], [125], [137] \\
\hline & & & Nanoparticles & {$[145-147]$} \\
\hline & & \multirow{6}{*}{$\begin{array}{c}\text { Particle } \\
\text { engineering }\end{array}$} & Additive: e.g. amino acid, lactose fine, surfactant & [122], [148-150] \\
\hline & & & Co-spray drying & [126], [151, 152] \\
\hline & & & $\begin{array}{c}\text { Micronization by milling, spray drying, spray-freeze } \\
\text { drying, in-situ-micronization }\end{array}$ & {$[102,103],[143],[153-156]$} \\
\hline & & & Direct crystallization & {$[157]$} \\
\hline & & & Antisolvent precipitation, micro- or nano-size & {$[158-160]$} \\
\hline & & & Solution enhanced dispersion by supercritical fluids & {$[115],[161]$} \\
\hline \multirow{9}{*}{ 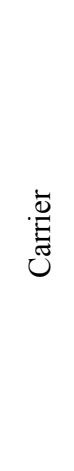 } & \multirow{9}{*}{ 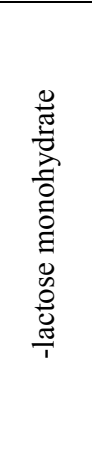 } & \multirow{5}{*}{$\begin{array}{l}\text { Physicochemic } \\
\text { al properties }\end{array}$} & Size and size distribution, polydispersity, fine excipient & {$[84],[162-165]$} \\
\hline & & & Morphology & {$[35,36],[88],[108]$} \\
\hline & & & Surface roughness & {$[117],[166]$} \\
\hline & & & Surface area & {$[110]$} \\
\hline & & & Surface energy & {$[110,111],[167]$} \\
\hline & & Surface coating & Force control agents & [90], [111], [116], [168, 169] \\
\hline & & Surface etching & Surface smoothing, surface area reduction & {$[91,92]$} \\
\hline & & $\begin{array}{c}\text { Particle } \\
\text { engineering }\end{array}$ & $\begin{array}{c}\text { Lactose composite, crystallization, spray-drying, } \\
\text { ultrasound assisted crystallization }\end{array}$ & {$[100],[36],[170-174]$} \\
\hline & & & Particle engineering review, e.g. spray drying & [93], [172], [94] \\
\hline \multirow{2}{*}{\multicolumn{2}{|c|}{$\begin{array}{l}\text { Alternative } \\
\text { carrier }\end{array}$}} & $\begin{array}{l}\text { Physicochemic } \\
\text { al properties }\end{array}$ & $\begin{array}{c}\text { Reducing or non-reducing sugar: mannitol, glucose, } \\
\text { erythritol, xylitol, raffinose, sucrose, maltitol, } \\
\text { maltodextran }\end{array}$ & $\begin{array}{c}{[105,106],[112],[121],[144],} \\
{[117],[175-177]}\end{array}$ \\
\hline & & & Sugar fines & {$[86],[176]$} \\
\hline $\begin{array}{r}\text { com } \\
\text { of }\end{array}$ & $\begin{array}{l}\text { ation } \\
\text { ove }\end{array}$ & $\begin{array}{l}\text { Review or } \\
\text { overview }\end{array}$ & $\begin{array}{l}\text { Relevant variables for in vitro aerosol resuspension from } \\
\text { adhesive mixture }\end{array}$ & $\begin{array}{c}\text { [178], [52], [49](review), } \\
{[179] \text { (review), [180, 181], }} \\
{[182](\text { review) }}\end{array}$ \\
\hline \multirow{3}{*}{\multicolumn{2}{|c|}{$\begin{array}{l}\text { Pharmaceutica } \\
1 \text { processing }\end{array}$}} & & Milling & {$[104]$} \\
\hline & & & Mixing & [111], [167], [183, 184] \\
\hline & & & Filling & {$[185]$} \\
\hline \multirow{4}{*}{\multicolumn{2}{|c|}{$\begin{array}{l}\text { Ternary } \\
\text { inclusion }\end{array}$}} & Intrinsic fines & Introduced by pharmaceutical processing & {$[98]$} \\
\hline & & Added fines & Lactose, alternative sugar, drug & $\begin{array}{c}87](\text { review), [176], [114], [186- } \\
188]\end{array}$ \\
\hline & & "Active" site & Carrier surface occupancy preferred at "active" sites & {$[189-191]$} \\
\hline & & agglomeration & Drug-fine large agglomerates easy to resuspense & [86], [113], [162], [192-194] \\
\hline \multicolumn{2}{|c|}{$\begin{array}{l}\text { Relative } \\
\text { humidity }\end{array}$} & $\begin{array}{l}\text { Storage effect, } \\
\text { humidity }\end{array}$ & Influence of moisture adsorption and ingress & [116], [170, 175], [194-196], \\
\hline
\end{tabular}


Figure 1-1. Mechanisms of Action of Drugs for Asthma Therapy.[19]

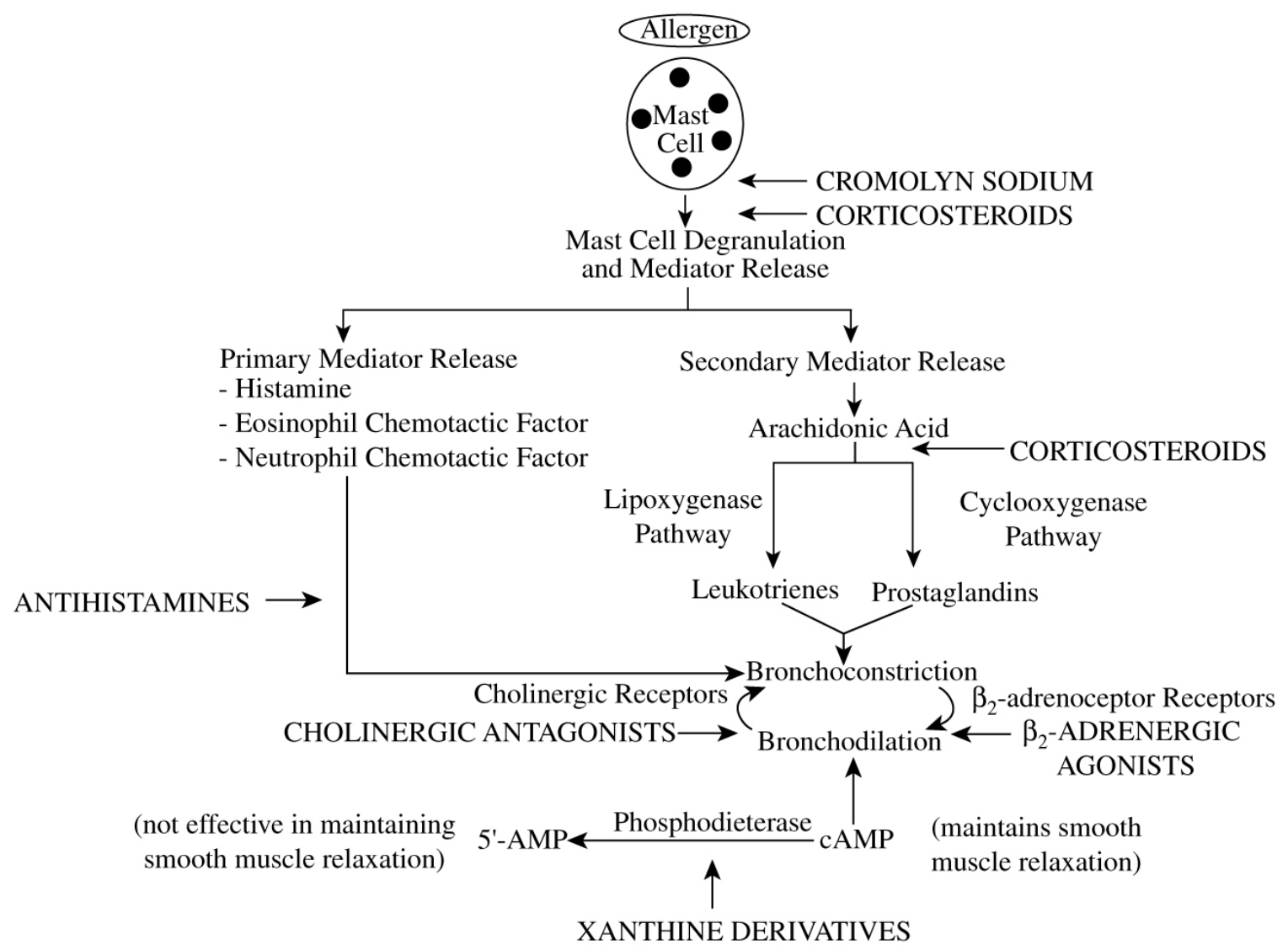


Figure 1-2. Schematic Diagrams of the Dry Powder Inhalers: (A) Rotahaler, a unit-dose low shear device; (B) Inhalator, a unit-dose high shear device; and (C) The various transition stages of powder aerosolization, through dilated, free flowing, and finally dispersed powder. (Courtesy of Dr. A.J. Hickey)

(A)

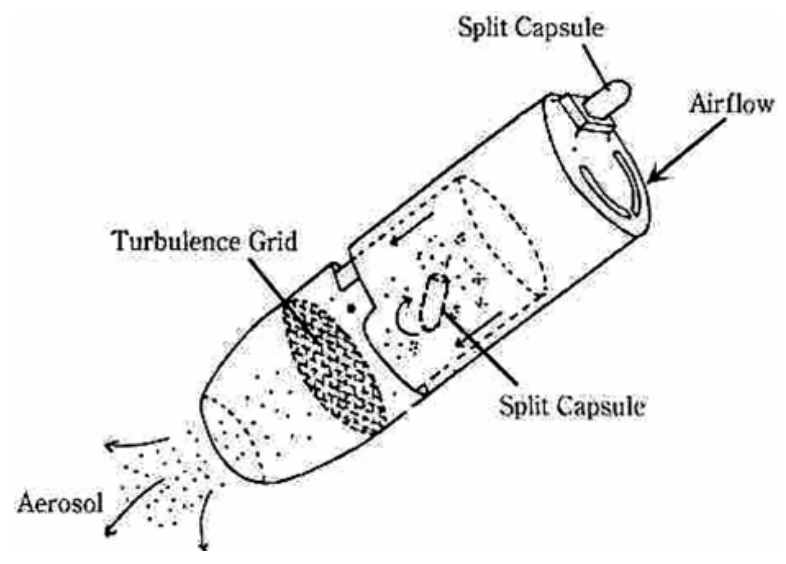

(B)

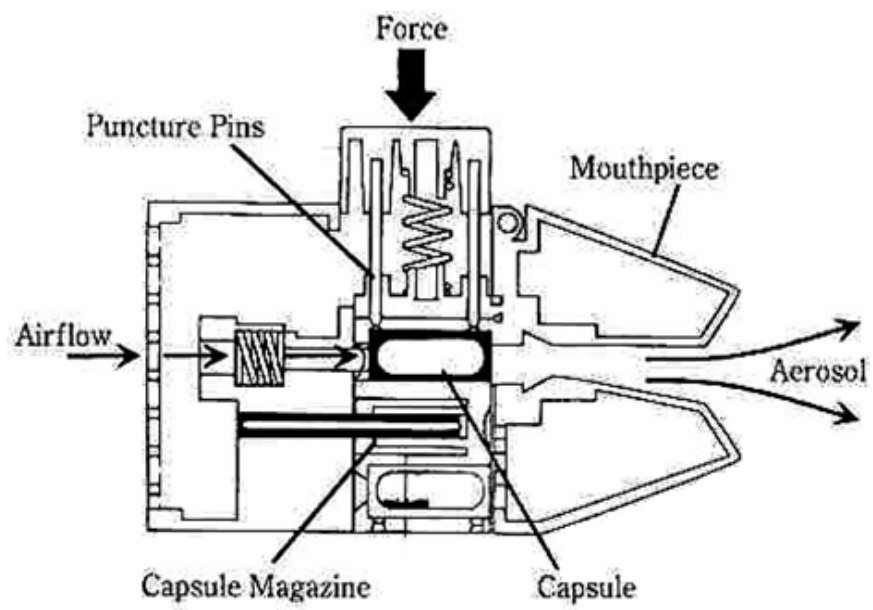

(C)

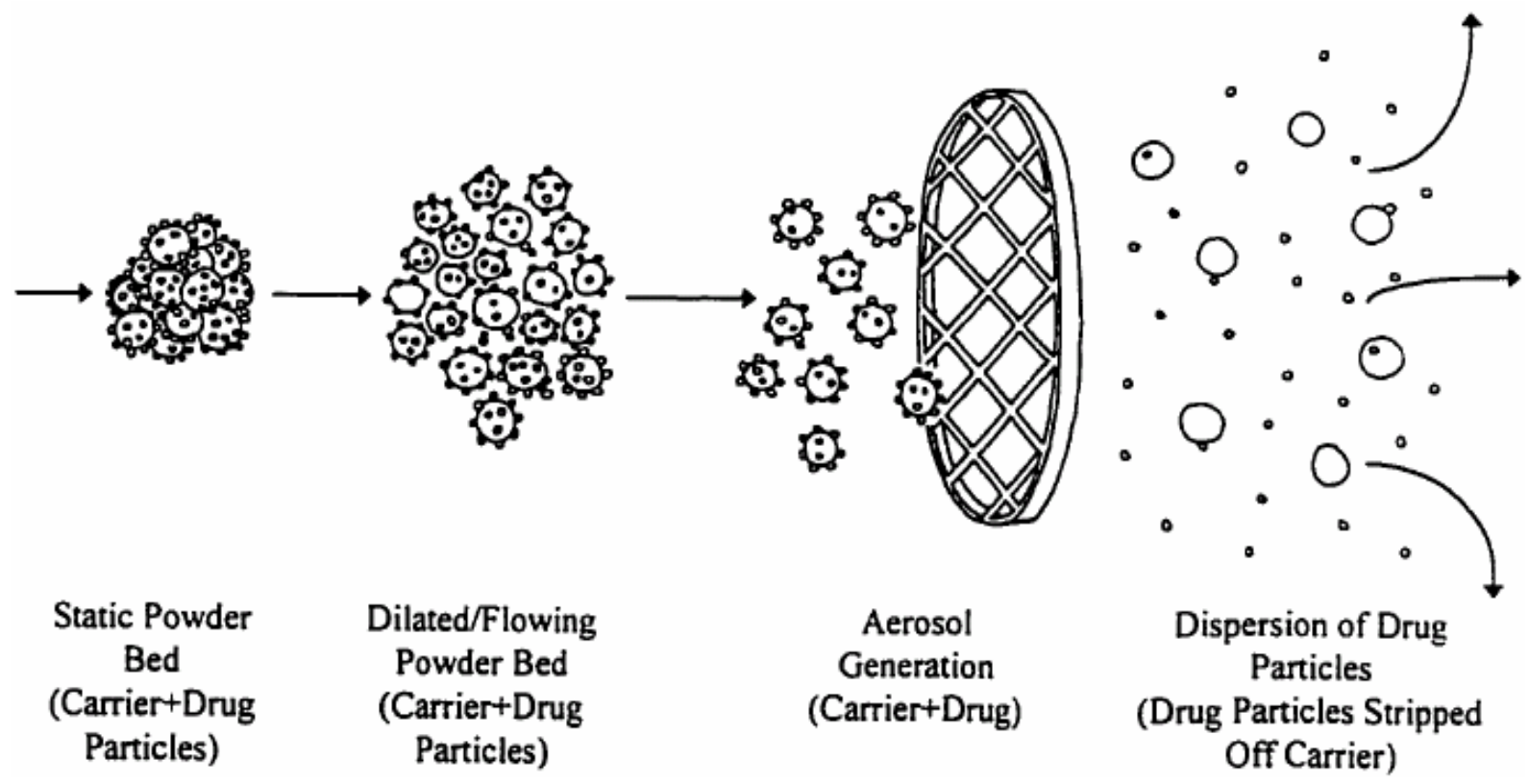


Figure 1-3. The Magnitude of Interparticulate Forces: (A) Adhesion forces; (B) Aerodynamic deaggregation forces.[73]

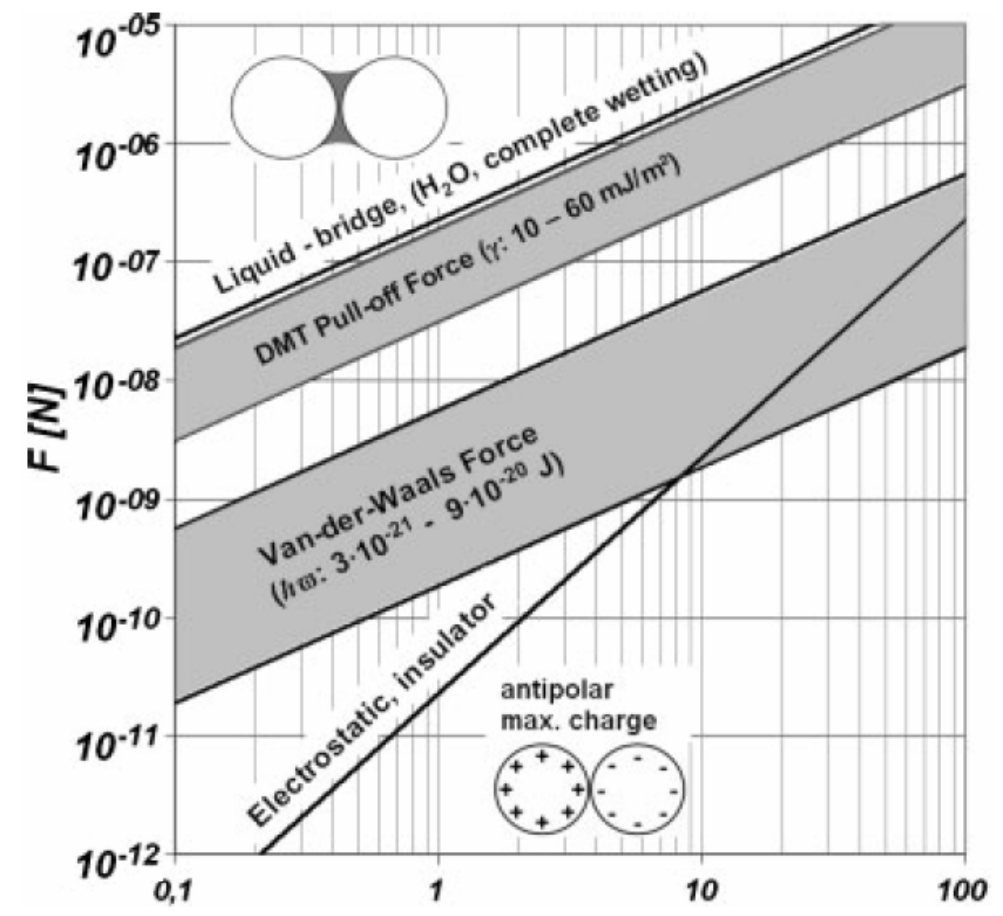

(B)

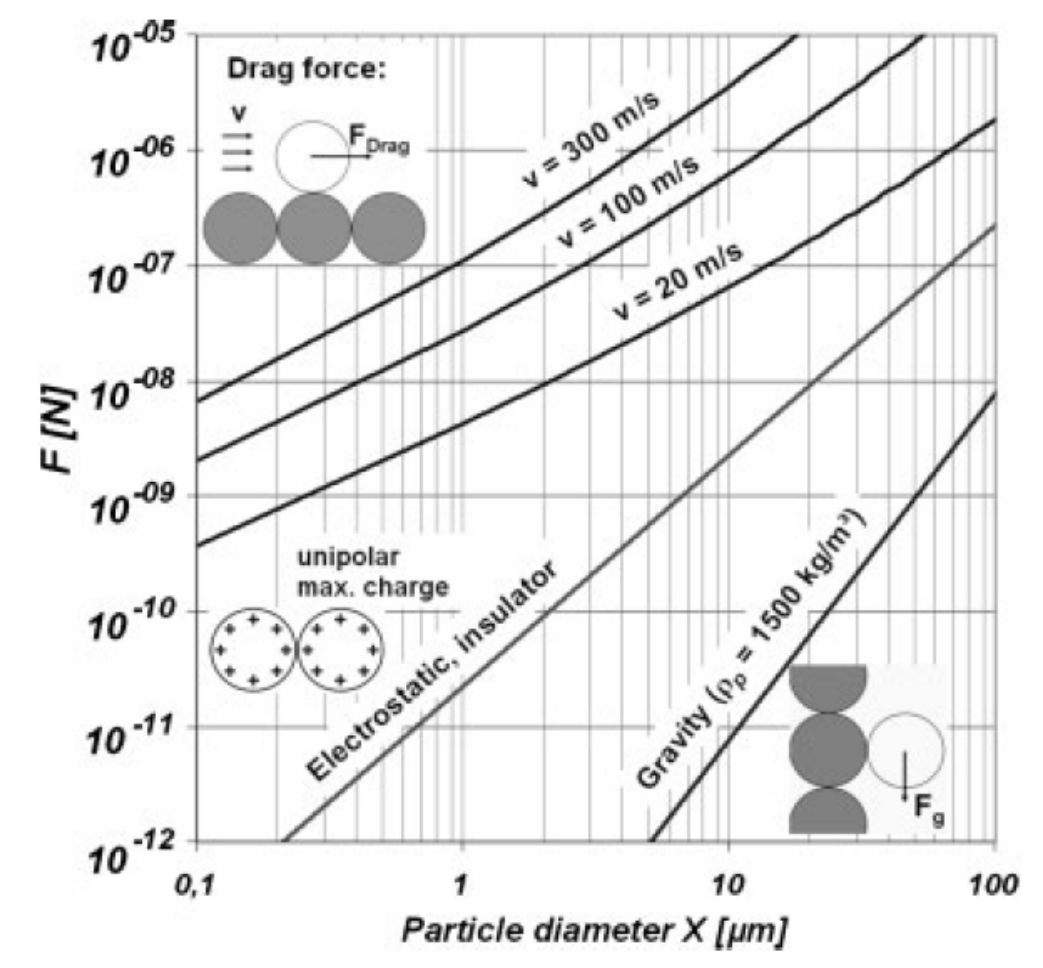




\section{References}

1. A.J. Hickey, Inhalation Aerosols: Physical and Biological Basis for Therapy. Marcel Dekker, New York (1996).

2. C. O'Callaghan, O. Nerbrink and M. Vidgren, in: Drug Delivery to the Lung, H. Bisgaard, C. O'Callaghan, and G. Smaldone, Vol. 162, pp. 1-20. Marcel Dekker, New York (2001).

3. T.M. Crowder, M.D. Louey, V.V. Sethuraman, H.D.C. Smyth and A.J. Hickey, Pharm Tech, 25, 99-113 (2001).

4. R.W. Niven and A.J. Hickey, in: Inhalation Aerosols: Physical and biological basis for therapy, A.J. Hickey, Vol. 221, pp. 253-283. Informa Healthcare USA, New York (2007).

5. The United States Pharmacopeia. U.S. Pharmacopeial Convention, Rockville, MD (2004).

6. A.J. Hickey and H.M. Mansour, in: Modified-release Drug Delivery Technology, M.J. Rathbone, et al., Vol. 2, pp. 573-602. Informa Healthcare, New York (2008).

7. $\quad$ Montreal Protocol on Substances that Deplete the Ozone Layers. (1987).

8. U.S. Food and Drug Administration, Use of Ozone-depleting Substances; Removal of Essential Use Designation. (2005).

9. P. Wright, in: Pharmaceutical Preformulation and Formulation: A Practical Guide from Candidate Drug Selection to Commercial Dosage Form, M. Gibson, Vol. 1, pp. 355-378. Taylor \& Francis Group, CRC Press, Boca Raton, FL (2004).

10. M.B. Chougule, B.K. Padhi, K.A. Jinturkar and A. Misra, Recent Patents on Drug Delivery \& Formulation, 1, 11-21 (2007).

11. H.M. Mansour, Int J Nanomedicine, 4, 299-319 (2009).

12. R. Dweik and J.K. Stoller, in: Egan's fundamentals of respiratory care, R.L. Wilkins, J.K. Stoller, and R.M. Kacmarek, Vol. pp. 503-524. Mosby, Inc., an affiliate of Elsevier Inc., St. Louis, Missouri (2009).

13. Centers for Disease Control and Prevention and N.C.f.H. Statistics. Asthma prevalence, health care use and mortality. 2006-cont. [cited; Available from: http://www.cdc.gov/nchs/fastats/asthma.htm.

14. P.J. Barnes, N Engl J Med, 321, 1517-27 (1989). 
15. P.J. Barnes, K.F. Chung and C.P. Page, Pharmacol Rev, 50, 515-96 (1998).

16. E.R. McFadden, Jr., in: Pulmonary diseases and disorders, A.P. Fishman, Vol. 1, pp. 1295-1310. McGraw-Hill Book Co., New York (1988).

17. P.J. Barnes, Chest, 117, 10S-4S (2000).

18. D.S. Gardenhire, in: Egan's Fundamentals of Respiratory Care, R.L. Wilkins, J.K. Stoller, and R.M. Kacmarek, Vol. 1, pp. 667-692. Mosby, Inc., an affiliate of Elsevier Inc., St. Louis, Missouri (2009).

19. A.J. Hickey, in: Drug Delivery: Principles and Applications, B. Wang, T. Siahaan, and R.A. Soltero, Vol. 1, pp. 341-361. John Wiley \& Sons, New Jersey (2005).

20. D.S. Gardenhire, in: Rau's Respiratory Care Pharmacology, D.S. Gardenhire, Vol. 1, pp. 103-131. Mosby, Inc., an affiliate of Elsevier Inc., St. Louis, Missouri (2008).

21. M.K. Taylor, Particle engineering to achieve optimal morphology and physicochemical characteristics for aerosol delivery of ipratropium bromide, Ph. D. Thesis, University of North Carolina, Chapel Hill (2001).

22. H. Kerstjens, D. Postma and N. ten Hacken, Clin Evid, 1923-47 (2005).

23. D.S. Gardenhire, in: Rau's Respiratory Care Pharmacology, D.S. Gardenhire, pp. 204-225. Mosby, Inc., an affiliate of Elsevier Inc., St. Louis, Missouri (2008).

24. D.S. Gardenhire, in: Rau's Respiratory Care Pharmacology, D.S. Gardenhire, pp. 150-161. Mosby, Inc., an affiliate of Elsevier Inc., St. Louis, Missouri (2008).

25. W.C. Hinds, Aerosol Technology: Properties, Behavior, and Measurement of Airborne Particles. John Wiley \& Sons, New York (1999).

26. C. Wang, in: Inhaled Particles, (Eds.), Vol. 1, pp. 31-54. Elsevier Ltd., London (2005).

27. T.M. Crowder, A.J. Hickey, M.D. Louey and O. N., A Guide to Pharmaceutical Particulate Science. Interpharm Press/CRC, Boca Raton, FL (2003).

28. T.M. Crowder, J.A. Rosati, J.D. Schroeter, A.J. Hickey and T.B. Martonen, Pharm Res, 19, 239-45 (2002).

29. I. Gonda, in: Pharmaceutical Inhalation aerosol technology, A.J. Hickey (Eds.), Vol. pp. 65-88. Marcel Dekkar, New York (2004).

30. C. Wang, in: Inhaled Particles, Vol. 1, pp. 55-78. Elsevier Ltd., London (2005). 
31. K. Ikegami, Y. Kawashima, H. Takeuchi, H. Yamamoto, N. Isshiki, D. Momose and K. Ouchi, Pharm Res, 19, 1439-1445 (2002).

32. H.K. Chan and I. Gonda, J Pharm Sci, 78, 176-80 (1989).

33. H.K. Chan and I. Gonda, J Pharm Sci, 84, 692-6 (1995).

34. K. Ikegami, Y. Kawashima, H. Takeuchi, H. Yamamoto, K. Mimura, D. Momose and K. Ouchi, J Control Release, 88, 23-33 (2003).

35. X.M. Zeng, A.P. Martin, C. Marriott and J. Pritchard, Int J Pharm, 200, 93-106 (2000).

36. H. Larhrib, G.P. Martin, C. Marriott and D. Prime, Int J Pharm, 257, 283-296 (2003).

37. J.H. Bell, P.S. Hartley and J.S. Cox, J Pharm Sci, 60, 1559-64 (1971).

38. P.J. Atkins, Respir Care, 50, 1304-1312 (2005).

39. N.R. Labiris and M.B. Dolovich, Br J Clin Pharmacol, 56, 600-612 (2003).

40. I.J. Smith and M. Parry-Billings, Pulm Pharmacol Ther, 16, 79-95 (2003).

41. S.P. Newman, Expert Opin Biol Ther, 4, 23-33 (2004).

42. D. Prime, P.J. Atkins, A. Slater and B. Sumby, Adv Drug Deliv Rev, 26, 51-58 (1997).

43. N. Islam and E. Gladki, Int J Pharm, 360, 1-11 (2008).

44. A.J. Hickey and T.M. Crowder, in: Inhalation Aerosols: Physical and biological basis for therapy, A.J. Hickey, Vol. 221, pp. 445-460. Informa Healthcare USA, Inc., NY (2007).

45. H.K. Chan, Expert Opin on Ther Patents, 13, 1333-1343 (2003).

46. C.A. Dunbar, A.J. Hickey and H. P., Kona, 16, 7-45 (1998).

47. R.C. Rowe, P.J. Sheskey and S.C. Owen, in: Handbook of Pharmaceutical Excipients, R.C. Rowe, P.J. Sheskey, and S.C. Owen, Vol. 1, pp. 385-398. Pharmaceutical Press and American Pharmacists Association, Washington, DC (2006).

48. A.J. Hickey and D. Ganderton, Pharmaceutical Process Engineering. Marcel Dekker, New York (2001).

49. H. Smyth and A.J. Hickey, Am J Drug Deliv, 3, 117-132 (2005). 
50. P.W. Barry and C. O'Callaghan, Adv Drug Deliv Rev, 55, 879-923 (2003).

51. S.P. Newman, Curr Opin Pulm Med, 9, S17-20 (2003).

52. F. Podczeck, Int J Pharm, 160, 119-130 (1998).

53. Y.J. Son and J.T. McConville, Drug Dev Ind Pharm, 34, 948-959 (2008).

54. R. Dalby and J. Suman, Adv Drug Deliv Rev, 55, 779-791 (2003).

55. A.H. de Boer, P. Hagedoorn, D. Gjaltema, J. Goede and H.W. Frijlink, Int J Pharm, 310, 72-80 (2006).

56. M.D. Louey, M. VanOort and A.J. Hickey, J. Aerosol Sci., 37, 1520-1533 (2006).

57. B.Y. Shekunov, J.C. Feeley, A.H. Chow, H.H. Tong and P. York, J Aerosol Sci, 34, 553-568 (2003).

58. A.H. de Boer, P. Hagedoorn, D. Gjaltema, J. Goede and H.W. Frijlink, Int J Pharm, 260, 187-200 (2003).

59. S.C. Nichols and E. Wynn, RDD 2008, 1, 175-184 (2008).

60. F. Podczeck, Particle-particle adhesion in pharmaceutical powder handling. Imperial College Press, London, UK (1998).

61. D.J. Griffiths, Introduction to Quantum Mechanics. Benjamin Cummings, (2004).

62. J.N. Israelachvili, Intermolecular and Surface Forces. Academic Press, London, UK (1992).

63. A.W. Adamson and A.P. Gast, Physical chemistry of Surfaces. John Wiley \& Sons, Inc., NY (1997).

64. P.C. Hiemenz, Principles of Colloid and Surface Chemistry. Marcel Dekker, New York (1977).

65. A. Castellanos, Adv. Phys., 54, 263-376 (2005).

66. H.P. Dunbar CHA, Kona, 16, 7-45 (1998).

67. H.Y. Erbil, Surface chemistry: solid and liquid interfaces. Blackwell publishing Ltd, Malden, MA (2006).

68. K. Kendall and C. Stainton, Powder Tech., 121, 223-229 (2001). 
69. J. Visser, Particulate Sci. Technol., 13, 169-196 (1995).

70. W.H. Finlay, The Mechanics of Inhaled Pharmaceutical Aerosols: An Introduction. Academic Press, London, UK (2001).

71. B.V. Derjaguin, Kolloid Z., 69, 155-164 (1934).

72. D.D. Do, Adsorption analysis: equilibria and kinetics. Imperial College Press, London, UK (1998).

73. C. Weiler, M. Egen, M. Trunk and P. Langguth, J Pharm Sci, 99, 303-316 (2010).

74. N.M. Concessio and A.J. Hickey, Adv Drug Deliv Rev, 26, 29-40 (1997).

75. X.M. Zeng, G.P. Martin and C. Marriott, Particulate Interactions in Dry Powder Formulations for Inhalation. Taylor \& Francis, New York (2001).

76. K.L. Johnson, K. Kendall and A.D. Roberts, Proc. R. Soc. London A, 324, 301-313 (1971).

77. B.V. Derjaguin, B.W. Muller and Y.P. Toporov, J. Colloid Interface Sci., 53, 314-325 (1975).

78. D. Tabor, J. Colloid Interface Sci., 58, 2-13 (1977).

79. A.J. Hickey, H.M. Mansour, M.J. Telko, Z. Xu, H.D. Smyth, T. Mulder, R. McLean, J. Langridge and D. Papadopoulos, J Pharm Sci, 96, 1302-1319 (2007).

80. J.R. Coury and M.L. Aguiar, Powder Tech., 85, 37-43 (1995).

81. D.L. French, D.A. Edwards and R.W. Niven, J. Aerosol Sci., 27, 769-783 (1996).

82. H. Smyth, in: Excipient Development for Pharmaceutical, Biotechnology, and Drug Delivery Systems, A. Katdare and M.V. Chaubal, Vol. 1, pp. 225-249. Informa Healthcare USA, New York (2006).

83. Z. Xu, H.M. Mansour and A.J. Hickey, J Adhesion Sci Tech, In peer review (2010).

84. H. Steckel and B.W. Muller, Int J Pharm, 154, 31-37 (1997).

85. F. Vanderbist, B. Wery, I. Moyano-Pavon and A.J. Moes, J Pharm Pharmacol, 51, 1229-34 (1999).

86. H. Adi, I. Larson and P.J. Stewart, Int J Pharm, 337, 229-238 (2007).

87. M.D. Jones and R. Price, Pharm Res, 23, 1665-1674 (2006). 
88. Y. Kawashima, T. Serigano, T. Hino, H. Yamamoto and H. Takeuchi, Int J Pharm, 172, 179-188 (1998).

89. A.D. Zimon and T.S. Volkova, Colloid J. USSR (English translation), 27, 306-307 (1965).

90. P. Begat, R. Price, H. Harris, D.A. Morton and J.N. Staniforth, Kona, 23, 109-121 (2005).

91. K. Iida, Y. Hayakawa, H. Okamoto, K. Danjo and H. Leuenberger, Chem Pharm Bull (Tokyo), 51, 1-5 (2003).

92. L.W. Chan, L.T. Lim and P.W. Heng, J Pharm Sci, 92, 975-984 (2003).

93. A.H. Chow, H.H. Tong, P. Chattopadhyay and B.Y. Shekunov, Pharm Res, 24, 411437 (2007).

94. R. Vehring, Pharm Res, 25, 999-1022 (2008).

95. E.N. Hiestand, Mechanics and Physical Principles for Powders and Compacts. SSCI, West Lafayette, IN (2002).

96. A.J. Hickey, H.M. Mansour, M.J. Telko, Z. Xu, H.D. Smyth, T. Mulder, R. McLean, J. Langridge and D. Papadopoulos, J Pharm Sci, 96, 1282-1301 (2007).

97. M. Gotzinger and W. Peukert, Langmuir, 20, 5298-303 (2004).

98. M.D. Louey, S. Razia and P.J. Stewart, Int J Pharm, 252, 87-98 (2003).

99. P.M. Young, M.J. Tobyn, R. Price, M. Buttrum and F. Dey, J Pharm Sci, 95, 18001809 (2006).

100. P.M. Young, P. Kwok, H. Adi, H.K. Chan and D. Traini, Pharm Res, 26, 802-810 (2009).

101. P. Begat, D.A. Morton, J.N. Staniforth and R. Price, Pharm Res, 21, 1826-1833 (2004).

102. M.D. Louey, M. Van Oort and A.J. Hickey, Pharm Res, 21, 1200-1206 (2004).

103. M.D. Louey, M. Van Oort and A.J. Hickey, Pharm Res, 21, 1207-1213 (2004).

104. P.M. Young, H.K. Chan, H. Chiou, S. Edge, T.H. Tee and D. Traini, J Pharm Sci, 96, 1331-1341 (2007).

105. J.C. Hooton, M.D. Jones and R. Price, J Pharm Sci, 95, 1288-1297 (2006). 
106. H. Steckel and N. Bolzen, Int J Pharm, 270, 297-306 (2004).

107. H. Adi, D. Traini, H.K. Chan and P.M. Young, J Pharm Sci, 97, 2780-2788 (2008).

108. P.W.S. Heng, L.W. Chan and L.T. Lim, Chem Pharm Bull (Tokyo), 48, 393-398 (2000).

109. P.M. Young, D. Cocconi, P. Colombo, R. Bettini, R. Price, D.F. Steele and M.J. Tobyn, J Pharm Pharmacol, 54, 1339-1344 (2002).

110. D. Cline and R. Dalby, Pharm Res, 19, 1274-1277 (2002).

111. M. Kumon, M. Suzuki, A. Kusai, E. Yonemochi and K. Terada, Chem Pharm Bull (Tokyo), 54, 1508-1514 (2006).

112. D. Traini, P.M. Young, F. Thielmann and M. Acharya, Drug Dev Ind Pharm, 34, 992-1001 (2008).

113. M.D. Jones, J.C. Hooton, M.L. Dawson, A.R. Ferrie and R. Price, Pharm Res, 25, 337-348 (2008).

114. N. Islam, P. Stewart, I. Larson and P. Hartley, Pharm Res, 21, $492-499$ (2004).

115. P.M. Young and R. Price, Eur J Pharm Sci, 22, 235-240 (2004).

116. K. Iida, Y. Hayakawa, H. Okamoto, K. Danjo and H. Luenberger, Chem Pharm Bull (Tokyo), 52, 444-446 (2004).

117. M.P. Flament, P. Leterme and A. Gayot, Int J Pharm, 275, 201-209 (2004).

118. M. Bunker, M. Davies and C. Roberts, Expert Opin Drug Deliv, 2, 613-24 (2005).

119. P. Begat, D.A. Morton, J.N. Staniforth and R. Price, Pharm Res, 21, 1591-1597 (2004).

120. D. Traini, P. Rogueda, P. Young and R. Price, Pharm Res, 22, 816-825 (2005).

121. M.D. Jones, H. Harris, J.C. Hooton, J. Shur, G.S. King, C.A. Mathoulin, K. Nichol, T.L. Smith, M.L. Dawson, A.R. Ferrie and R. Price, Eur J Pharm Biopharm, 69, 496507 (2008).

122. N.Y. Chew, B.Y. Shekunov, H.H. Tong, A.H. Chow, C. Savage, J. Wu and H.K. Chan, J Pharm Sci, 94, 2289-2300 (2005). 
123. P.M. Young, S. Edge, D. Traini, M.D. Jones, R. Price, D. El-Sabawi, C. Urry and C. Smith, Int J Pharm, 296, 26-33 (2005).

124. K. Iida, Y. Hayakawa, H. Okamoto, K. Danjo and H. Luenberger, Chem Pharm Bull (Tokyo), 49, 1326-1330 (2001).

125. F. Buttini, P. Colombo, M.P. Wenger, P. Mesquida, C. Marriott and S.A. Jones, $J$ Pharm Sci, 97, 1257-1267 (2008).

126. D. Lechuga-Ballesteros, C. Charan, C.L. Stults, C.L. Stevenson, D.P. Miller, R. Vehring, V. Tep and M.C. Kuo, J Pharm Sci, 97, 287-302 (2008).

127. A.R. Clark and A.M. Hollingsworth, J Aerosol Med, 6, 99-110 (1993).

128. T. Srichana, G.P. Martin and C. Marriott, Eur J Pharm Sci, 7, 73-80 (1998).

129. N.M. Concessio, M.M. VanOort, M.R. Knowles and A.J. Hickey, Pharm Res, 16, 828-34 (1999).

130. M.S. Coates, H.K. Chan, D.F. Fletcher and J.A. Raper, Pharm Res, 22, 1445-53 (2005).

131. M.S. Coates, D.F. Fletcher, H.K. Chan and J.A. Raper, Pharm Res, 22, 923-32 (2005).

132. A.H. de Boer, P. Hagedoorn, D. Gjaltema, J. Goede and H.W. Frijlink, Int J Pharm, 310, 81-89 (2006).

133. M. Jaroniec and R. Madey, Physical Adsorption on Heterogeneous Solids. Elsevier Science Publishers, New York (1988).

134. Z. Xu, H.M. Mansour, T. Mulder, R. McLean, J. Langridge and A.J. Hickey, Respiratory Drug Delivery, 3, 897-900 (2008).

135. N.Y. Chew, D.F. Bagster and H.K. Chan, Int J Pharm, 206, 75-83 (2000).

136. N.Y. Chew and H.K. Chan, J Pharm Pharm Sci, 5, 162-168 (2002).

137. K.A. Fults, I.F. Miller and A.J. Hickey, Pharm Dev Technol, 2, $67-79$ (1997).

138. N.Y. Chew, P. Tang, H.K. Chan and J.A. Raper, Pharm Res, 22, 148-152 (2005).

139. C. Weiler, M. Egen, M. Trunk and P. Langguth, J Pharm Sci, (2009).

140. D. El-Sabawi, R. Price, S. Edge and P.M. Young, Drug Dev Ind Pharm, 32, 243-251 (2006). 
141. N.Y. Chew and H.K. Chan, Pharm Res, 18, 1570-1577 (2001).

142. S. Adi, H. Adi, P. Tang, D. Traini, H.K. Chan and P.M. Young, Eur J Pharm Sci, 35, 12-18 (2008).

143. K. Gilani, A.R. Najafabadi, M. Barghi and M. Rafiee-Tehrani, J Pharm Sci, 94, 10481059 (2005).

144. J.C. Hooton, M.D. Jones, H. Harris, J. Shur and R. Price, Drug Dev Ind Pharm, 34, 974-983 (2008).

145. A.S. Cantor, J.S. Stefely, P.A. Jinks, J.R. Baran, J.M. Ganser, M.W. Mueting, B.J. Gabrio and S.W. Stein, Respiratory Drug Delivery, 1, 309-318 (2008).

146. M. Linsenbuhler and K.E. Wirth, Powder Technol., 158, 3-20 (2005).

147. Y. Kawashima, T. Serigano, T. Hino, H. Yamamoto and H. Takeuchi, Pharm Res, 15, 1748-1752 (1998).

148. K. Giry, J.M. Pean, L. Giraud, S. Marsas, H. Rolland and P. Wuthrich, Int J Pharm, 321, 162-166 (2006).

149. P. Lucas, K. Anderson, U.J. Potter and J.N. Staniforth, Pharm Res, 16, 1643-1647 (1999).

150. H. Steckel and H.G. Brandes, Int J Pharm, 278, 187-195 (2004).

151. D.O. Corrigan, O.I. Corrigan and A.M. Healy, Int J Pharm, 322, 22-30 (2006).

152. H. Adi, P.M. Young, H.K. Chan, P. Stewart, H. Agus and D. Traini, J Pharm Sci, 97, 3356-3366 (2008).

153. G.S. Zijlstra, W.L. Hinrichs, A.H. de Boer and H.W. Frijlink, Eur J Pharm Sci, 23, 139-149 (2004).

154. H. Steckel, N. Rasenack, P. Villax and B.W. Muller, Int J Pharm, 258, 65-75 (2003).

155. H. Steckel, N. Rasenack and B.W. Muller, Eur J Pharm Biopharm, 55, 173-180 (2003).

156. S.P. Duddu, S.A. Sisk, Y.H. Walter, T.E. Tarara, K.R. Trimble, A.R. Clark, M.A. Eldon, R.C. Elton, M. Pickford, P.H. Hirst, S.P. Newman and J.G. Weers, Pharm Res, 19, 689-695 (2002).

157. D. Murnane, G.P. Martin and C. Marriott, J Pharm Sci, 98, 503-515 (2008). 
158. R. Westmeier and H. Steckel, J Pharm Sci, 97, 2299-2310 (2008).

159. Bhavna, F.J. Ahmad, G. Mittal, G.K. Jain, G. Malhotra, R.K. Khar and A. Bhatnagar, Eur J Pharm Biopharm, 71, 282-291 (2009).

160. N. El-Gendy, E.M. Gorman, E.J. Munson and C. Berkland, J Pharm Sci, 98, 27312746 (2009).

161. H. Schiavone, S. Palakodaty, A. Clark, P. York and S.T. Tzannis, Int J Pharm, 281, 55-66 (2004).

162. F. Podczeck, Aerosol Sci. Technol., 31, 301-321 (1999).

163. P. Lucas, Proc Respiratory Drug Delivery, 6, 243-250 (1998).

164. N.M. Kassem and D. Ganderton, J Pharm Pharmacol, 41, 14P (1989).

165. T. Srichana, G.P. Martin and C. Marriott, Int J Pharm, 167, 13-23 (1998).

166. K. Iida, Y. Hayakawa, H. Okamoto, K. Danjo and H. Leuenberger, Chem Pharm Bull (Tokyo), 53, 431-434 (2005).

167. I. Saleem, H. Smyth and M. Telko, Drug Dev Ind Pharm, 34, 1002-1010 (2008).

168. K. Iida, Y. Hayakawa, H. Okamoto, K. Danjo and H. Luenberger, Chem Pharm Bull (Tokyo), 52, 350-353 (2004).

169. K. Iida, Y. Hayakawa, H. Okamoto, K. Danjo and H. Luenberger, Chem Pharm Bull (Tokyo), 51, 1455-1457 (2003).

170. P. Harjunen, V.P. Lehto, K. Martimo, E. Suihko, T. Lankinen, P. Paronen and K. Jarvinen, Eur J Pharm Sci, 16, 313-321 (2002).

171. R.S. Dhumal, S.V. Biradar, A.R. Paradkar and P. York, Pharm Res, 25, 2835-2844 (2008).

172. P.C. Seville, H.Y. Li and T.P. Learoyd, Crit Rev Ther Drug Carrier Syst, 24, 307-360 (2007).

173. P. Young, D. Roberts, H. Chiou, W. Rae, H.K. Chan and D. Traini, J Aerosol Sci, 39, 82-93 (2008).

174. X. Ming Zeng, G.P. Martin, C. Marriott and J. Pritchard, Eur J Pharm Biopharm, 51, 55-62 (2001). 
175. X.M. Zeng, H.B. MacRitchie, C. Marriott and G.P. Martin, Int J Pharm, 333, 45-55 (2007).

176. S.K. Tee, C. Marriott, X.M. Zeng and G.P. Martin, Int J Pharm, 208, 111-123 (2000).

177. D. Traini, P.M. Young, M. Jones, S. Edge and R. Price, Eur J Pharm Sci, 27, 243-251 (2006).

178. A.H. de Boer, P. Hagedoorn, D. Gjaltema, J. Goede, K.D. Kussendrager and H.W. Frijlink, Int J Pharm, 260, 201-216 (2003).

179. H.K. Chan and N.Y. Chew, Adv Drug Deliv Rev, 55, 793-805 (2003).

180. N.Y. Chew and H.K. Chan, J Aerosol Med, 15, 325-330 (2002).

181. X.M. Zeng, G.P. Martin, C. Marriott and J. Pritchard, J Pharm Pharmacol, 52, 1211$1221(2000)$.

182. H.K. Chan, J Aerosol Med, 19, 21-27 (2006).

183. K. Iida, Y. Inagaki, H. Todo, H. Okamoto, K. Danjo and H. Luenberger, Chem Pharm Bull (Tokyo), 52, 938-942 (2004).

184. M. Kumon, S. Machida, M. Suzuki, A. Kusai, E. Yonemochi and K. Terada, Chem Pharm Bull (Tokyo), 56, 617-625 (2008).

185. F. Podczeck, Int J Pharm, 178, 93-100 (1999).

186. M.D. Louey and P.J. Stewart, Pharm Res, 19, 1524-1531 (2002).

187. X.M. Zeng, G.P. Martin, C. Marriott and J. Pritchard, J Pharm Sci, 90, 1424-1434 (2001).

188. K. Iida, H. Leuenberger, L.M. Fueg, R. Muller-Walz, H. Okamoto and K. Danjo, Yakugaku Zasshi, 120, 113-119 (2000).

189. X.M. Zeng, K.H. Pandhal and G.P. Martin, Int J Pharm, 197, 41-52 (2000).

190. X.M. Zeng, G.P. Martin, S.K. Tee, A.A. Ghoush and C. Marriott, Int J Pharm, 182, 133-144 (1999).

191. X.M. Zeng, G.P. Martin, S.K. Tee and C. Marriott, Int J Pharm, 176, 99-110 (1998).

192. P. Lucas, K. Anderson and J.N. Staniforth, Pharm Res, 15, 562-569 (1998). 
193. H. Adi, I. Larson, H. Chiou, P. Young, D. Traini and P. Stewart, J Pharm Sci, 97, 3140-3152 (2008).

194. S. Das, I. Larson, P. Young and P. Stewart, J Pharm Sci, 98, 1015-1027 (2009).

195. P.M. Young, R. Price, M.J. Tobyn, M. Buttrum and F. Dey, Drug Dev Ind Pharm, 29, 959-966 (2003).

196. R.N. Jashnani and P.R. Byron, Int J Pharm, 130, 13-24 (1996). 


\section{CHAPTER 2 LACTOSE CHARACTERIZATION}

\subsection{Introduction}

\subsubsection{Lactose Monohydrate as Carrier}

Lactose [1] is the most widely used pharmaceutical excipient in solid and liquid dosage forms and is the only approved DPI carrier in the US, although the investigation of alternative DPI carriers is ongoing. As a carrier in DPI formulation, it functions as a fluidizing agent in the drug aerosolization and as a bulking agent for accurate powder filling and metering. Lactose has a well established safety profile, is inexpensive, and abundant since it is derived from milk and the dairy industry. It has several desirable physicochemical properties (monohydrate crystalline form) such as relative smooth surface, non-hygroscopic (critical relative humidity $\sim 99 \%$ at $24{ }^{\circ} \mathrm{C}$ [2]), moderate interparticulate interactions and flow properties.[3]

Lactose is a disaccharide that consists of galactose and glucose bonded through a $\beta-1 \rightarrow 4$ glycosidic linkage. The most common chemical form of lactose used as pharmaceutical excipient is the crystalline $\alpha$-lactose monohydrate $\left(\mathrm{C}_{12} \mathrm{H}_{22} \mathrm{O}_{11} \cdot \mathrm{H}_{2} \mathrm{O}\right)$, which is prepared by crystallization from supersaturated aqueous solution below 93.5 ${ }^{\circ} \mathrm{C}$.[4] The other two chemical forms of lactose are anhydrous $\alpha$-lactose and $\beta$-lactose. The former can be obtained by removing the bound water of $\alpha$-lactose monohydrate, which can be achieved either by heat treatment or anhydrous methanol treatment of $\alpha$-lactose monohydrate.[5] The latter is the anomer of $\alpha$-form because of mutarotation in 
solution, the stereoisomers inter-conversion at anomeric position. The equilibrium concentration of the two anomers is dependent on temperature, solvent, and $\mathrm{pH}$. It should be noted that all pharmaceutical crystalline $\alpha$-lactose monohydrate contain inherent $\beta$-lactose impurity to a different extent, because the nucleation and growth of the former under nonequilibrium concentrations of the latter.[6] On the basis of modeling calculation, it has been proposed that the characteristic tomahawk shape of crystalline $\alpha$-lactose monohydrate arises as a consequence of non-uniform incorporation of the $\beta$-lactose into the growing interface of particular growth sectors. $[6,7]$ The accurate quantification of $\beta$-lactose can be achieved by derivatization of anomeric proton by trimethylsilyl group followed by gas chromatography.[8] As a source of milk product, the surface of the crystalline $\alpha$-lactose monohydrate may contain tiny amount of residual contaminants such as protein (casein, lactoalbumin, and whey) and lipid (triglycerides and fatty acids),[1, 9] which may be beneficial or become drawbacks for use.

For the same chemical form of lactose, the thermal properties including melting point, heat of fusion, and heat capacity could be different. For example, the thermal properties of anhydrous $\alpha$-lactose produced by methanol treatment of crystalline $\alpha$-lactose monohydrate are different from that formed by heat.[5] This is the behavior of different molecular packing to form different the solid state. Lactoses with different degrees of molecular disorder can be prepared by a few methods. For example, a molecularly smooth surface, with Miller indices for the $\left\{\begin{array}{lll}0 & 1 & 1\end{array}\right\}$ face of $\alpha$-lactose monohydrate crystals can be prepared by temperature controlled micro-crystallization.[10] The pure amorphous lactose can be produced by spray drying under the condition 
described by Lloyd et al.[11] The physical characteristics of $\alpha$-lactose monohydrate such as morphology, size, and $\beta$-lactose content could also vary if the nucleation and crystallization condition such as supersaturation, temperature, and impurity content change.[6] Other properties such as Young's modulus vary greatly for $\alpha$-lactose monohydrate $(24.1 \mathrm{GPa}), \beta$-lactose $(17.9 \mathrm{GPa})$, and spray dried lactose (11.4 GPa).[12] Furthermore, the manufacturing (e.g. flash drying) and pharmaceutical processing may mechanically induce various degrees of molecular disorders on the surface of the crystalline $\alpha$-lactose monohydrate. These molecular disorders can also influence processing, storage, and delivery characteristics because they are usually thermodynamically unstable. At elevated moisture (water as plasticizer to lower glass transition temperature) or heat exposure, the activation energy barrier towards recrystallization could be significantly lowered with serious implications with regards to long-term stability and batch-to-batch variations.[13] The amount of surface amorphous contents could be small and undetectable by standard analytical techniques, but it could profoundly change the surface energy distribution, interparticulate interactions, and eventually the aerosolization performance.[14-17] Staniforth [18] reviewed the influence of different forms of lactose to the aerosolization properties of the formulation. The results showed the $\beta$-lactose was easily entrained, but bound the drug particles most strongly. The anhydrous $\alpha$-lactose showed the opposite behavior and the $\alpha$-lactose monohydrate showed intermediate behavior. However, anhydrous $\alpha$-lactose is highly hygroscopic. Because the vast difference of these physicochemical properties, the production of inhalation grade lactose should be carefully selected and controlled because 
it could eventually lead to significant therapeutic and biological effects. Lactose monohydrate may function in interactive physical mixtures or in a particle matrix that contain homogenously dispersed active drug at molecular or particle level. This dissertation will focus on the former, the interactive physical mixtures. The objective of this Chapter is to characterize the physicochemical properties of lactose monohydrate batches and their interactive physical mixtures with micronized model drug albuterol sulfate.

\subsubsection{Techniques for DPI Formulation Characterization}

\subsubsection{Static powder characterization}

The physicochemical characterization of DPI formulation components (drug or carrier powder and the corresponding blends at rest) has been reviewed previously.[19] The classical physicochemical properties characterization of solid state material include scanning electron microscopy, differential scanning calorimetry, X-ray powder diffraction, atomic force microscopy, inverse gas chromatography, and laser diffraction.

Scanning electron microscopy (SEM) scans the sample surface with high-energy beams of electrons in a raster scanning mode. The electrons interact with surface atoms to produce high-resolution images of the sample surface topography, composition and other properties such as electrical conductivity. It is routinely used for particle size, morphology, and surface feature analysis of dry powder formulations. 
Differential scanning calorimetry (DSC) evolved from differential thermal analysis and share similar thermal analytical principles.[20] The basis of DSC measurement is depending on the specific heat (enthalpy: $\Delta \mathrm{H}$ ), the amount of sample (heat capacity: $\Delta \mathrm{C}_{\mathrm{p}}$ ), and the baseline deviation from that recorded with an empty sample holder. DSC can be applied to detect and quantify thermotropic phase transitions, phase behavior, and molecular interactions of powder formulation.[21] Either kinetic (fast) scan or thermodynamic (slow) scan is used to measure a number of physicochemical features including exothermic crystallization, recrystallization, decomposition, etc; or endothermic melting, desolvation, dehydration, glass transition, etc. Melting or crystallization undergoes first order transitions. The integration of the curve gives the energy involved in the transitions; whereas glass transition attributes to the second order transition, and the signal gives changes of the specific heat (shift of baselines).[22] Other applications of DSC include the analysis of amorphous solid state,[23] polymorphism,[24] isothermal analysis of oxidative reactions, and liquid crystals which belong to a state between anisotropic (solid) and isotropic (liquid) called meso-morphous state (e.g. some phospholipids).[25] Besides, DSC is also used as a quality control instrument for impurity detection and stability issues.

X-ray powder diffraction (XRPD) is widely used to determine the degree of longrange order and crystallinity of pharmaceutical powders. The theoretical basis of XRPD was developed by Hermans and Weidinger.[26] Briefly, the crystalline intensity and amorphous intensity should be able to distinguish from the powder pattern. Usually, both peak intensity and area intensity are measured. The diffraction patterns are then compared 
with a collection of single-phase X-ray powder patterns known as powder diffraction files (PDF) to determine the quality of the data. The PDF files are provided by several sources such as the University of Cambridge Database and the international center for diffraction data (ICDD, Newtown Square, PA).[27] The determination of amorphous content is based on the assumption that the proportionality exists between the experimentally measured amorphous intensity and the amorphous fraction in the standard sample.[28]

Atomic force microscopy (AFM) can be used to examine surface structures of a variety of materials with mesoscopic scale resolution $\left(10^{-6}-10^{-9} \mathrm{~m}\right)$ and quantify the individual particle pull-off forces by direct force measurement.[19] Basically, there are two primary operation modes: the contact mode and non-contact (tapping) mode. The contact mode uses the tip deflection as a feedback signal, whereas the non-contact mode detects the oscillation change due to cantilever tip-sample interaction when the cantilever is oscillated close to its resonance frequency. The oscillation change provides information about the sample surface characteristics (topography and phase). There are several factors that link the seemingly straightforward AFM surface topography and force measurement. They include: the cantilever tip consistency, the physical and chemical properties of colloid probe and the substrate surfaces, the environmental issues, and the contact area determination or normalization.[19] The surface roughness can be characterized by the root mean square deviation $\left(\mathrm{R}_{\mathrm{rms}}\right)$ in a given area. The adhesion relies on the true contact area of interactions which are influenced by the surface roughness. The adhesion forces distributed more widely when a rough surface was used because the asperity radius or the effective contact area is more scattered.[29-31] 
Laser diffraction (LD) is a commonly used light scattering methods of particle sizing based on either Frauhofer or Mie interpretation.[32] A laser source is applied (e.g. Helium-Neon laser, $682.3 \mathrm{~nm}, 5 \mathrm{~mW}$ ) to the scattered powder sample to create a diffraction pattern. A large fraction of the power scattered by small particles is scattered into large angles, while a large fraction of the powder scattered by large particles is scattered into the near forward-direction. A set of concentric annular rings perpendicular to the axis of the laser beam forms the detector array to detect the light scattering of the sample and intensity of the light collected on each rings will determine the number of particles in the defined size range. Statistical analysis results in a combination of frequency and cumulative distribution curves from which the volume diameters $\left(D_{10}, D_{50}\right.$, and $\left.D_{90}\right)$ and span $\left(\operatorname{span}=\left(D_{90^{-}} D_{10}\right) / D_{50}\right)$ of the scattered particles can be obtained. The advantage of this technique is rapid measurement of particle volume size distribution. However, it is not possible to differentiate between drug and carrier particle.[33]

\subsubsection{Aerodynamic characterization (powder resuspension)}

The aerodynamic properties of powders including flow properties (characterized by $E D$ ) and deaggregation (characterized by $F P F$ or $F P M$ ) may be evaluated in vitro by a variety

of techniques generally described as inertial sampling techniques.[33] The inertial sampling theory is based on the Stokes's terminal settling velocity and curvilinear particle motion. Two devices, the twin-stage liquid impinger and Andersen cascade impactor, and their deposition efficiency are depicted in Figure 2-1 [33]. 
Twin-stage liquid impinger (TSLI) was introduced in the late 1980s [34] and included in British Pharmacopoeial standard in 1998 (Figure 2-1A).[35] It uses two stages to collect dispersed particles, with the cutoff diameter of $6.4 \mu \mathrm{m}$ at $60 \mathrm{~L} / \mathrm{min}$ airflow rate. The impaction surface is formed by the perpendicular between the airflow and the surface of the collection solution. Volumes of 7 and $30 \mathrm{~mL}$ are placed in stages 1 and 2, respectively. The collection efficiency curve for TSLI is broad (Figure 2-1C), but it resembles the oropharyngeal deposition curve that allows quick characterization of aerodynamic properties of aerosols.

Based on the inertial sampling principle, particles whose inertia exceeds a certain value are unable to follow the streamline, and impacted (deposited) on the impaction plate. Andersen non-viable cascade impactor (ACI) operates several impactor stages (up to 10 stages are often used) with decreasing aerodynamic cutoff diameter, $d_{50}$, in series (Figure 2-1B). The sequential separation divides the particle distribution into a series of contiguous groups according to their aerodynamic diameters. Therefore, the aerosolization performance (ED, FPF, FPM, and MMAD) indicated by the aerodynamic size distribution can be obtained by statistically calculating the deposited fractions. ACI is more discriminating and precise than the TSLI. The collection efficiencies (Figure 2-1D) of each stage can be calibrated using monodispersed aerosols at defined airflow rate (typically at 28.3, 60, and $90 \mathrm{~L} / \mathrm{min}$.[36] It should be noted that in vitro ACI performance study does not truly represent lung deposition. Unlike the lungs that have rapid expanding surface area with descending generations, the surface areas of each ACI stages are similar. Unlike the upper airway such as oropharyngeal and trachea that have turbulent airflow, 
the airflows in the acinar airways (respiratory bronchioles, alveolar ducts, and alveolar sac) are slow and laminar $(\operatorname{Re}<2000)$. However, the sampling velocity and Reynolds number of ACI increases for successive stages (so that successively smaller particles could be collected).[37]

\subsection{Material and Methods}

\subsubsection{Material}

Lactose monohydrate (Respitose ${ }^{\mathrm{TM}}$ ) batches were obtained from DMV-Fonterra Excipients. These batches consisted of six sieved batches (SV-A to SV-F) and two milled batches (ML-A and ML-B). These batches were selected from initially 35 batches lactose monohydrate based on two principal components analyses and cluster analyses performed independently. These analyses were used to assess the diversity of batches with respect to characteristic such as particle size, bulk and tap density, water activity, etc. The physical properties of these lactose monohydrates were supplied by the manufacturer and published previously (Table 2-1).[38] Micronized albuetrol sulfate (AS) was supplied by Pfizer Global R\&D (Kent, UK). It was used immediately after sieving to reduce agglomeration due to storage.

\subsubsection{Blend preparation}


AS is used as the drug probe for physicochemical and performance evaluation of the lactose monohydrate-based formulation. Micronized AS was allowed to pass through a $125 \mu \mathrm{m}$ sieve to reduce the degree of agglomeration. Two drug concentrations $(0.5$ and 2 $\% \mathrm{w} / \mathrm{w}$ ) of micronized AS in lactose monohydrate interactive physical mixtures were prepared. A preblend of AS (10 or $40 \mathrm{mg}$ ) with lactose monohydrate batches (1990 or $1960 \mathrm{mg}$ ) was prepared by geometrical addition in an aluminum foil container using a spatula. The preblends were further processed in a Turbula ${ }^{\circledR}$ mixer (Model T2C, GlenMills, Inc., Clifton, NJ) for 20 min. Blend uniformity was evaluated by analyzing five samples of each mixture taken from different regions of the blends. The amounts of AS in the blends were quantified by UV absorption spectroscopy measured at a wavelength of $\lambda=225$ (Shimadzu UV-1700 UV/Vis spectrometer, Shimadzu Corporation, Columbia, MD) in deionized water (Barnstead Nanopure, 18.0 M 2 ). In all cases, a coefficient of variation $(\% \mathrm{CV})<5.0 \%$ was considered homogeneous. The prepared interactive physical mixtures were placed in tightly sealed glass jars (Wheaton Science Products, Millville, NJ) which were then stored in glass desiccators (Drierite $\AA /$ Indicating Drierite ${ }^{\circledR}$ ) under ambient temperature and pressure prior to further characterization and aerosolization performance study.

\subsubsection{Analytical Approaches for Lactose Batches and Blends}

\subsubsection{Size distribution, morphology, and surface features (SEM)}


The volume size, morphology, surface features and blend uniformity of drugs, lactose monohydrate batches and corresponding blends were examined by SEM. The samples were sputter-coated with Au-Pd in Argon for $90 \mathrm{~s}$ before the experiment (Polaron 5200 sputter coater, West Chester, PA). About 150-250 $\AA$ of Au-Pd thin films could be coated on the sample surface. Scanning electron micrographs (Jeol JSM 6300V SEM, Peabody, $\mathrm{NY})$ at a number of magnifications $(\times 150, \times 600, \times 1.5 \mathrm{k}, \times 3 \mathrm{k}, \times 6 \mathrm{k}, \times 10 \mathrm{k}$ and $\times 20 \mathrm{k})$ were examined for micronized albuterol sulfate, and at magnifications $(\times 60, \times 150, \times 600, \times 1.5 \mathrm{k})$ for all lactose monohydrate batches and drug/lactose monohydrate blends.

\subsubsection{Thermal properties (DSC)}

The molecular and thermotropic phase transitions of each individual component including micronized AS, lactose monohydrate batches, and interactive physical mixtures were examined by DSC (Perkin Elmer DSC 6 Workstation, Norwalk, CT). Non-hermetically sealed aluminum pans containing 1.5-6.0 mg of accurately weighed samples were used. Nitrogen purge gas $\left(\mathrm{N}_{2}\right.$ prepurified, Airgas National Welders Supply, Charlotte, NC) was set at $20 \mathrm{~mL} / \mathrm{min}$. The temperature and heat flow were calibrated using high purity Indium (m.p. $=156.60{ }^{\circ} \mathrm{C}, \Delta \mathrm{H}_{\mathrm{f}}=28.45 \mathrm{~J} / \mathrm{g}$ ) and Zinc (m.p. $=419.47{ }^{\circ} \mathrm{C}, \Delta \mathrm{H}_{\mathrm{f}}=108.37$ $\mathrm{J} / \mathrm{g}$ ). Various heating rates, including $2.0{ }^{\circ} \mathrm{C} / \mathrm{min}, 5.0{ }^{\circ} \mathrm{C} / \mathrm{min}$, and $10.0{ }^{\circ} \mathrm{C} / \mathrm{min}$, were applied to examine the thermotropic phase behavior $\left(\Delta \mathrm{H}, \mathrm{T}_{\mathrm{m}}\right)$ and kinetic/metastable phase transitions such as glass transitions $\left(\mathrm{C}_{\mathrm{p}}, \mathrm{T}_{\mathrm{g}}\right)$. Thermograms were processed and analyzed using the accompanying software (Pyris Thermal Analysis Instrument Control and Data Analysis Software, v.3.01). 


\subsubsection{Crystallinity (XRPD)}

The degree of crystallinity and non-crystallinity of the drugs before and after milling, lactose monohydrate batches and interactive mixtures was examined by slit detector XRPD. (Regaku Multiflex, Tokyo, Japan). The powder was packed onto an etched square area of XRPD glass slide that was placed horizontally in the sample holder. Diffractograms were obtained to evaluate the crystallinity of the samples $(1-2 \mathrm{~g})$. The scanning was at $12 \theta$ degree/min and from 3-60 $2 \theta$ degree at room temperature $\left(23{ }^{\circ} \mathrm{C}\right)$ to cover angles and nanometer distances where peaks occur for pharmaceutical material. The intensity of the peaks and the area under the curves were compared with the University of Cambridge XRPD database to estimate the long-range and short-range order of the material.

\subsubsection{Particle volume size distribution (LD)}

The volume size distribution of drugs was characterized by Fraunhofer laser diffraction (Malvern Instruments 2600 Series, Worcs, UK) using micronized solid drug particles suspended in an organic dispersant. The focal length of the lens is $63 \mathrm{~mm}$, giving a nominal size range of $0.5-118 \mu \mathrm{m}$. The organic dispersant was chloroform, which is commonly used as a dispersing liquid in surface chemical and interfacial experiments due to its favorable combination of hydrophobicity and low viscosity. The solid-liquid dispersion was sonicated briefly $(20 \mathrm{~s})$ to eliminate any small aggregates while not physically breaking the primary particles (ie. particle fracture) and modifying the primary particle size. 


\subsubsection{Surface Topography (AFM)}

Nanotopographic images of individual lactose monohydrate surfaces (SV and ML) were acquired using the Topometrix Explorer AFM (ThermoMicroscopes, Sunnyvale, CA) under ambient conditions $\left(23-25{ }^{\circ} \mathrm{C} ; 35-40 \%\right.$ RH). Steel stubs (Ted Pella, Inc.) were used for mounting samples. Cantilever was mounted using superglue. Samples were mounted using MikroStik $^{\mathrm{TM}}$ (Ted Pella, Inc.). Silicon nitride cantilever tips (non-contact tips without coating) were PPP-NCL (Nanosensors ${ }^{\mathrm{TM}}$, Neuchatel, Switzerland) with the following specifications: Thickness: $7 \mu \mathrm{m}$ (range: 6-8 $\mu \mathrm{m}$ ); Length: $225 \mu \mathrm{m}$ (range: 215-235 $\mu \mathrm{m}$ ); Force constant: $48 \mathrm{~N} / \mathrm{m}$ (range: 21-98 N/m); Average resonance frequency: $190 \mathrm{kHz}$. Scan rates were applied at 5 and $10 \mu \mathrm{m} / \mathrm{s}$ in non-contact acquisition mode. Scan ranges were $50 \mu \mathrm{m} \times 50 \mu \mathrm{m}, 10 \mu \mathrm{m} \times 10 \mu \mathrm{m}$ and $5 \mu \mathrm{m} \times 5 \mu \mathrm{m}$, respectively. Images with resolution set at 100, 300, 400, and 500 were obtained. Best images were obtained by using a combination of a slower scanning rate and higher resolution. The entire AFM was place onto a bungee suspension to reduce the low frequency vibration noise. ThermoMicroscopes SPM lab analysis software (ThermoMicroscopes) and Gwyddion software were used in analyzing the AFM images (some topography images and data processing were generated using Asylum Reseach MFP3D AFM). Sample preparation was carried out by mounting to steel discs at or near their plane of maximum stability by the following procedure: A small amount of powder was dropped from a height $(0.5 \mathrm{~m})$ onto clean overhead projector transparencies; then powder sample discs were painted with MikroStik ${ }^{\mathrm{TM}}$ adhesive until excess solvent had visibly evaporated; after that the disc was inverted (adhesive side down) on a position on the transparency that contained a 
dilute region of powder; finally the un-adhered particles were removed by tapping of disc on bench. This method achieves good particle dilution, and a good probability for particles to adhere with large surfaces facing up.

\subsubsection{In Vitro Performance Assessment (TSLI)}

\subsubsection{Standardized Entrainment Tubes (SETS)}

Four SETs (denoted as SET-A, B, C, and D) with airflow parameters encompassing those of commercial DPI devices were selected. The possible mechanisms of particle separation in the SETs include 1) particle entrainment in a laminar or turbulent shear fluidization; 2) particle entrainment in a laminar or turbulent wall boundary layer; 3) turbulent deaggregation of agglomerates by aerodynamic forces or turbulent transient acceleration (relative motion) and; 4) particle detachment by momentum transfer (e.g. impaction and vibration). Although all of these mechanisms could occur to a different extent during the powder resuspension, the aerodynamic contribution can be represented by four airflow parameters (Reynolds number, pressure drop, power, and shear stress) at defined airflow rate. (Table 1-1 [39]). The pressure drop, $\Delta P$, is directly related to specific resistance $\left(R_{D}\right)$ (Eq. 2-1):[40]

$$
\sqrt{\Delta P}=R_{D} \cdot Q \quad \text { (Eq. 2-1) }
$$

where $Q$ is the volumetric flow rate. The $R_{D}$ is dependent on the geometry and dimension of the airflow path, which is an intrinsic parameter of the SETs. Higher $R_{D}$ values are expected to correspond to greater turbulence and deaggregation, but too high $R_{D}$ values 
will cause difficulty in inhalation maneuver. The Reynolds number (Re) of the SETs represents the ratio of inertial forces to the viscous forces (Eq. 2-2):[41]

$$
\mathrm{Re}=\frac{F_{\text {inertial }}}{F_{\text {friction }}}=\frac{\rho \cdot d \cdot V}{\mu}
$$

where $\rho$ is the density of air $\left(1.20 \mathrm{~kg} / \mathrm{m}^{3}\right.$ at $\left.20{ }^{\circ} \mathrm{C}\right) ; V$ is the linear velocity; $d$ is the inner diameter of the SET; and $\mu$ is the dynamic viscosity of air $\left(1.81 \times 10^{-5} \mathrm{~kg} /(\mathrm{m} \mathrm{s})\right)$. Reynolds number is a dimensionless number used to predict the transition from laminar to turbulent flow. The laminar flow is present at Re values smaller than 2100 , whereas the turbulent flow is present at Re values greater than 4000.[41] Re also represents a measure of shear forces experienced with the SETs. Higher Re values correspond to higher shear forces.[39] The power is the rate of work done by the airflow through the entrainment tube (Eq. 2-3):[42]

$$
\text { Power }=\Delta P \cdot Q \quad \text { (Eq. 2-3) }
$$

The shear stress $\tau_{s}$ (Eq. 2-4, where $\mu$ is the dynamic viscosity of air; $V$ is the velocity, $y$ is the distance to the wall) is probably the most important parameter that acts on the particle deaggregation in the turbulent air stream. Shear stress represents the force required for one layer of fluid to slide over another layer. The viscous shear stress for turbulent airflow can be calculated from a nozzle flow applied from Kolmogorov turbulence theory (Eq. 2-5), where $\mu$ and $v$ are the dynamic and kinematic viscosity of air, respectively; $\varepsilon$ is the mean turbulent energy dissipation rate; and $d$ the nozzle diameter.[43]

$$
\tau_{s}=\mu \frac{\partial V}{\partial y} \quad(\text { Eq. 2-4) }
$$




$$
\tau_{s}=\mu\left(\frac{\varepsilon}{v}\right)^{1 / 2} \quad \text { where } \varepsilon \cong \frac{2\langle V\rangle^{3}}{d} \approx \frac{2(0.2 V)^{3}}{d}
$$

The powder formulation ( $25 \mathrm{mg} /$ actuation) was transferred onto the loading area, which was at the proximal end of the inlet section, prior to acturation. The loading area of SET-A and B refers to the peripheral wall, whereas a centrally located platform is employed for SET-C and D. Such designs maximize airflow velocity and turbulent shear stress. When the inlet and outlet sections were connected, the SET was held horizontally and checked using a bubble-level, and placed into the mouthpiece of commonly used inertial impaction apparatus such as TSLI or ACI. In order to evaluate the multiplicity of factors that influenced the aerosol formulation performance, the aerosolization was experimentally designed, the number of each experiment was randomly assigned (Design Expert 5.0.9 software, Stat-Ease Corp., MN), and performed at fixed airflow rate $(Q=60$ L/min). In vitro aerosolization was evaluated under ambient condition. (RH\%: 23-44, temp: $23-24{ }^{\circ} \mathrm{C}$ )

\subsubsection{Twin-stage liquid impinger (TSLI)}

The SETs were connected directly at the inlet port of the TSLI. Both with and without solenoid switch actuation were assessed. For the performance studies with solenoid switch, the switch was placed between the TSLI and the vacuum pump to achieve instant actuation. After each actuation ( $25 \mathrm{mg}$ formulation, $10 \mathrm{sec}$ ), deposited AS fractions were collected by washing the SET and each portion of the TSLI with deionized water, and analyzed by UV-spectrometer $(\lambda=225)$. The aerodynamic behavior of selected formulations was evaluated by emitted dose (ED: percentage of drug emitted from total 
dose), fine particle fraction (or absolute mass) from total dose $\left(F P F_{T D}\right.$ : percentage of mass (or absolute mass, $F P M_{T D}$ ) of drug below $6.4 \mu \mathrm{m}$ cut-off size from total dose), and fine particle fraction (or absolute mass) from emitted dose $\left(F P F_{E D}\right.$ : percentage of mass (or absolute mass, $F P M_{E D}$ ) of drug below $6.4 \mu \mathrm{m}$ cut-off size from emitted dose). Three SETs (denoted as SET-A, C, and D) with airflow parameters encompassing those of commercial DPI devices were selected. Their airflow parameters $\left(R e, \Delta P\right.$, power, and $\left.\tau_{s}\right)$ at airflow $Q=60 \mathrm{~L} / \mathrm{min}$ were shown in Table 1-1.[39] Moreover, in order to evaluate the multiplicity of factors including drugs, lactose monohydrate, and airflow conditions that influenced the formulation performance, full factorial $\left(2^{3}\right)$ experimental analysis was designed, performed, and interpreted statistically (Design Expert 5.0.9 software, Stat-Ease Corp., MN)

\subsubsection{Andersen non-viable cascade impactor (ACI)}

Similar to TSLI studies, the aerosolization performance of selected formulations was also evaluated using eight-stage ACI ( $25 \mathrm{mg} /$ actuation $\times 2$ actuation $)$ at $60 \mathrm{~L} / \mathrm{min}$ airflow rate. The ACI cut-off diameters were calibrated previsouly (Table 2-2). Both with and without solenoid switch were evaluated (10 s per actuation). Before sampling, each stage of ACI was immersed into $0.1 \%$ silicone oil in hexanes and dried in ambient condition to avoid particle bouncing and reentrainment. A USP throat and preseparator were used. The quantification of the deposited AS was achieved by UV $(\lambda=225)$. The ED, FPF (or FPM), and MMAD were obtained by measuring and analyzing the quantity of AS deposited on each stage of ACI. The impactor wall loss was evaluated $(<5 \%)$ during method validation, but was not included in the designed experiment and calculation. 


\subsection{Results and Discussion}

\subsubsection{Aerosol Characterization}

\subsubsection{Particle size and morphology (SEM)}

Representative SEM images of eight batches of lactose monohydrate (SV-A to F and ML-A to B), the micronized drug AS, and their corresponding interactive physical mixtures are shown in Figure 2-2 and 2-3. The SV lactose monohydrates had more uniform particle shape, smoother surface, and had much fewer intrinsic fine particles (designated as particle diameter less than $10 \mu \mathrm{m}$ ) than ML lactose monohydrates, although the major particle size of both SV and ML batches were comparable. This was consistent with the LD analyses shown below. For ML batches, there were significantly more fine particle agglomerates and nanocrevices than SV batches. The micronized AS was in the respirable size range and narrow size distribution. The particles had relatively smooth surface textures with rectangular-shaped non-spherical particles. Particle agglomeration was observed at all magnification before blending (some agglomerates as large as $\sim 40 \mu \mathrm{m}$ ), indicating strong cohesive forces at the particle-particle interface. Image processing by modifying the threshold value indicated that more fine particle agglomerates in ML lactose monohydrates were observed to spread between the void instead of adhering on the surface of major particles.[19] It was hard to distinguish the fine lactose particles with the drug particles simply from the shape and morphology, especially ML lactose monohydrate containing many lactose fines. But there appeared to 
be an increase in particles in range of $1-10 \mu \mathrm{m}$. The increase in fine particles appeared to adhere more onto the major carrier particle surfaces, with more roughness or crevices, indicating "high surface energy" in these sites (Figure 2-2). The particle size, size distribution, morphology, and surface features were comparable between the six SV batches, and between the two ML batches. The SEM result was consistent with LD data.

The significant difference between SV and ML lactose monohydrate batches could change the aerosolization performance of the blends. The surface fines observed in ML could serve at an intrinsic ternary component to modify the interactions between the primary carrier particles and micronized drug particles. Either of the two ternary formulation hypotheses, "active" site mechanism or agglomeration mechanism (see Chapter 6), could contribute to improve the deaggregation during inhalation.

\subsubsection{Thermal Properties (DSC)}

Representative DSC thermograms are shown in Figure 2-4. Both SV and ML batches had an endothermic dehydration peak $\sim 144{ }^{\circ} \mathrm{C}$ and a melting peak $\sim 213{ }^{\circ} \mathrm{C}$, indicative of predominant $\alpha$-lactose monohydrate. The calculated $\Delta H$ of these two endothermic peaks were smaller for ML than SV. In both ML batches, there was a distinctive exothermic peak $\sim 173{ }^{\circ} \mathrm{C}(\Delta H$ of ML-A: $\sim-9.4 \mathrm{~J} / \mathrm{g} ;$ ML-B: $\sim-8.4 \mathrm{~J} / \mathrm{g})$ when $5{ }^{\circ} \mathrm{C} / \mathrm{min}$ scanning rate was applied. This observation is consistent with the phase transition from molecular disordered to ordered state (amorphous content in ML). Scanning rate dependence was observed. In SV batches, this characteristic exothermic peak was much smaller (SV-A) or undetectable $(\mathrm{SV}-\mathrm{B})$. The glass transition temperature $\left(\mathrm{T}_{\mathrm{g}}\right)$ was not observed for all 
lactose monohydrate batches. Neither was the b-lactose anomer (typically $\mathrm{T}_{\mathrm{m}} \sim 235^{\circ} \mathrm{C}[5]$ ) detected because of decomposition when using non-hermetically sealed pans. The heating rate at $5{ }^{\circ} \mathrm{C} / \mathrm{min}$ gave more accurate fusing temperature than higher heating rate (such as $10^{\circ} \mathrm{C} / \mathrm{min}$ ) when micronized AS was measured (Figure 2-4C and 4D). The micronized AS exhibited an endothermic peak $\left(T_{m}=192{ }^{\circ} \mathrm{C}, \Delta H \sim 184.9 \mathrm{~J} / \mathrm{g}\right)$ at $5{ }^{\circ} \mathrm{C} / \mathrm{min}$. The decomposition occurred over $210^{\circ} \mathrm{C}$, which was in agreement with previous reports.[44, 45]

Upon blending with AS, both endothermic peaks of all lactose monohydrate batches were broadened (indicated by the smaller onset and larger end temperature), particularly for the second endothermic peak. The exothermic peak intensity was increased and became noticeable for both SV batches, indicating an increased molecular disorder due to physical blending. The change in physical properties indicated by the thermograms of lactose monohydrates following interactive physical blending could affect the macroscopic aerosol performance.

\subsubsection{Crystallinity (XRPD)}

Representative XRPD data (courtesy of Dr. Heidi Mansour) of selected SV and ML batches were re-plotted using Match! ${ }^{\circledR}$ software, Crystal Impact, Inc., Germany [46]) (Figure 2-5A and 5B). The comparison of SV and ML diffractograms indicated that a higher degree of molecular disorder of the ML batches than SV batches, as was indicated by the reduced intensity of peaks observed in ML batches. No detectable $\beta$-lactose was observed for all lactose batches when compared with the XRPD from the University of 
Cambridge database (Figure 2-5C and 5D) and previous reports.[19, 47] The mechanical process such as blending led to a varying degree of disorder due to the formation of defects or amorphous regions. These regions were believed to be more sensitive to moisture or chemical changes. The XRPD of AS is shown in the next Chapter, when a total of four different drugs were characterized (see Figure 3-6).

\subsubsection{Particle size distribution (LD)}

The particle size distributions of all $6 \mathrm{SV}$ and $2 \mathrm{ML}$ lactose monohydrate batches are shown in Table 2-1 (data supplied by the manufacturer). SV lactose monohydrate batches exhibited more uniform particle size distributions than ML batches, as were indicated by the smaller span values (span for SV: 1.10 1.23; ML: 3.13-3.14). But the volume median diameters $(\sim 52-61 \mu \mathrm{m}$, typical for DPI carriers) for all batches were in a comparable size range. The particle size distribution results were in good agreement with SEM images. The particle size distribution of AS was unimodal with very narrow span $\left(\mathrm{D}_{50}=3.59 \pm\right.$ $0.13 \mu \mathrm{m} ; \operatorname{span}=0.26 \pm 0.09)$.

\subsubsection{Surface roughness (AFM)}

In order to gain a greater understanding of the surface feature of the lactose monohydrate batches, selected SV and ML batches were studied using AFM. The images were obtained using tapping mode AFM. Representative 3D images of SV and ML primary particle surfaces are shown in Figure 2-6. It appeared that the surface roughness of SV was mainly resulted in the fine particle attachment, whereas the surface roughness of ML could either due to the crevices or protrude of the primary particle surface, or due to the 
scanning across the attached fine particles. Both microroughess (Figure 2-6C) and macroroughness (Figure 2-6D) were observed in SV and ML lactose batches. The two types of roughness could lead to vast difference in particle adhesion. The surface roughnesses could be formed during mechanical sieving and milling process. In order to account for the surface heterogeneity due to geometric contribution, the topography data was processed to produce root mean square roughness $\left(R_{R M S}=\sqrt{\frac{1}{n} \sum_{i=1}^{n} y_{i}^{2}}\right)$ values, where $n$ is the number of points in topography profile and $y_{i}$ the distance of asperities $(i)$ from the center line. The result showed that the surface roughness among the three SV batches (SV-B, SV-C, and SV-F) in a $10 \mu \mathrm{m} \times 10 \mu \mathrm{m}$ scanning area were $507 \pm 269 \mathrm{~nm}, 803 \pm$ $187 \mathrm{~nm}, 704 \pm 148 \mathrm{~nm}$, respectively ( $n=3)$; and ML batch (ML-B) in a $20 \mu \mathrm{m} \times 20 \mu \mathrm{m}$ scanning area as $1.38 \pm 0.74 \mu \mathrm{m}$. From the images and analysis collected so far, it appeared that the ML batches (e.g. ML-B) had more irregular surface morphology and surface defects than SV batches. This is in good agreement with the SEM result. The scanning over the ML particle surface was sometimes more problematic, resulting in tip contamination by contact when the cantilever reaches the scanning capacity (Figure 2-6D), or tip-fine particle contamination when scanning across the region with loosely attached surface fines. Scanning at lower amplitudes with higher set-points may reduce the chance of tip-fine contamination, but it also decreased image resolution. In the literature, efforts to reduce the tip-fine contamination were made by removing surface fines (e.g. decanting, air-jet sieving, etc), but such treatment may alter the surface structure and adhesion forces prior to AFM experiment. 


\section{$\underline{\text { 2.3.2 In Vitro Aerosol Performance Assessment }}$}

\subsubsection{TSLI}

Four interactive physical mixtures of AS (two lactose monohydrate (SV131 and ML058) at two concentrations of AS $(0.5 \% \mathrm{w} / \mathrm{w}$ and $2 \% \mathrm{w} / \mathrm{w}))$ were used in the initial performance studies with solenoid switch. Three SETs (SET-A, C, and D) were employed. The performance data are shown in Appendix 2-A. The performance results (ED and $\left.\mathrm{FPF}_{\mathrm{TD}}\right)$ were plotted against SET parameters (pressure drop, Reynolds number, and shear stress) (Figure 2-7).[48] It indicated that there was a general trend that at lower values of SET parameters, the ED values were higher, but the FPF values were lower. The difference of ED across different formulations was larger at higher values of SET parameters. At higher values of SET parameters, there seem to be a level-off of FPF for ML formulations. SV formulations gave higher value of ED but lower value of FPF than ML formulations. For SV formulations, the FPF values were higher across the entire SET parameters at higher concentration. But this trend was not observed in ML formulations.

The results indicated that there was a trade-off between ED and FPF when SETs were used as the dispersion device. Higher ED values corresponded to lower FPF values. This could be explained by the capability of SETs to differentiate two portions of entrained powders: the portion undergoing deaggregation and the portion without undergoing deaggregation. This point would be discussed the next chapter, when comprehensive performance studies were discussed. When the performance data were 
plotted against different SET parameters (i.e. pressure drop, Reynolds number, and shear stress), the trend were similar. This implied that the characterization of SETs using these parameters reflected the turbulent shear forces which were the major forces for powder deaggregation. At higher range of SET parameters, the ML formulation performance had less variability as the parameters changed. This indicated the reproducibility of performance efficiency at different inhalation maneuver, which is a desirable feature for formulation development. Physicochemical characterization results showed that SV lactose monohydrate batches were more uniform in size and shape than ML batches. This difference may lead to improved flow properties of $\mathrm{SV}$ formulations than $\mathrm{ML}$ formulations. Thus, superior ED of SV formulations observed in performance studies across all SET airflow parameters. ML batches had more intrinsic fine particles and much larger span. ML also had more surface irregularity, roughness, and surface molecular disorder than SV. Yet, ML formulations had superior FPF to SV formulations. The improved aerosolization performance could be explained as the effect of ternary blending mechanisms (see Chapter 6). The intrinsic fine particles in ML batches could possibly acted as ternary components, either masked the "active" sites or contributed to drug-fine agglomerates formation, both of which were ternary blending mechanisms that improved performance. It also showed that higher FPF were observed when higher concentration of $\mathrm{SV}$ formulation was used. It could be that the small amount of fines observed in SV batches was not enough to saturate all the "active" sites. When AS was bound to these sites, its removal from these sites became difficult. But, when higher concentration of AS was used, the AS particle adhere to sites with decreasing adhesive forces, and resulted in 
increased FPF when the same removing forces were applied. However, the effect of drug concentration did not affect the performance in the ML formulation.

The effects of carrier (2 SV and 2 ML lactose monohydrate batches), drug concentration $(0.5 \% \mathrm{w} / \mathrm{w}$ and $2 \% \mathrm{w} / \mathrm{w})$, and SET (D for low shear, A for high shear) were further analyzed using DesignExpert analysis (Appendix 2B and 2C). The notable contributing variables for the $\mathrm{FPF}_{\mathrm{TD}}$ and $\mathrm{FPF}_{\mathrm{ED}}$ were lactoses and applied shears (SETs), while the drug concentration was not statistically significant contributor. The contributing variables for the ED were also not significant. In order to focus only the significant parameters, the drug concentration, from then on, would be fixed at $2 \% \mathrm{w} / \mathrm{w}$ for the studies in the next few chapters.

\subsubsection{ACI}

The aerosolization performance using ACI yielded similar results as the TSLI experiment (Figure 2-8).[48] ED values were higher for SV-0.5\%AS formulation, whereas the FPF values were higher for ML-2\%AS formulation. The FPF values are more important for the performance efficiency evaluation. It should be noted that when evaluating the particle size distribution of the aerodynamic deposition, the performance with solenoid switch vs. without solenoid switch gave totally different result. The performance with solenoid switch indicated that as shear stress increased, the MMAD decreased exponentially. Conversely, the performances without solenoid switch showed an initial increase at lower shear range, while decrease at the highest shear. It is known that MMAD is a fundamental aerodynamic parameter that represents the degree of 
deaggregation. Smaller MMAD values represented greater deaggregations. This indicated that the inclusion of solenoid switch was necessary for reproducible and meaningful performance studies. Instantaneous actuation (with solenoid switch) was necessary for consistent drug aerosolization performance studies across different shear stress. Only instantaneous actuation data gave consistently increased FPF and decreased MMAD across the increasing shear stress if they were correlated. From then on, the solenoid switch would be used for all the further aerosolization performance studies.

\subsection{Summary}

A comprehensive set of experimental techniques were applied to characterize bulk and surface properties of different batches of SV and ML lactose monohydrate. This included physicochemical characterizations and in vitro aerosolization performance using SETs and a probe drug AS. Both individual component (one-component systems) and the interactive mixtures (two-component systems) were examined. Some sub-studies including the selection of appropriate lactose monohydrate batches, the influence of using solenoid switch $v s$. without using it, the concentration influence of the drugs in the interactive physical mixtures, the dosing in the SETs for performance reproducibility, and the ACI wall loss estimation (reasonable omission) were all considered. All of these sub-studies have been pulled together and served for the further studies that would be covered in the next few chapters. The general conclusions for the studies of lactose monohydrate carriers are that SV lactose monohydrate batches are more uniform in size and morphology. ML lactose monohydrate batches had much more surface fine particles and 
molecular disorders (surface defects and amorphous content). The aerosolization performance studies using AS-ML formulations gave superior performance efficiency (higher FPF) to AS-SV formulations. The lactose carrier and SET parameters were significant contributors that influence aerosolization performance, whereas AS concentration was not always a significant contributor for performance. Solenoid switch was necessary for the performance reproducibility and interpretation.

In Chapter 3, the drugs in the formulation development will be focused on, and the effects of drugs, lactose monohydrate carriers, and SET shear stresses, respectively, will be discussed. Two ways forward for the formulation carrier development, either searching for alternative sugar carriers, or modifying current existing lactose monohydrate, will be discussed in Chapter 4 and Chapter 6, respectively. 
Table 2-1. Selected physicochemical properties of eight batches of lactose as supplied by the manufacturer.[38]

\begin{tabular}{|c|c|c|c|c|c|c|c|c|c|}
\hline \multicolumn{2}{|c|}{ Batch } & SV-A & SV-B & SV-C & SV-D & SV-E & SV-F & ML-A & ML-B \\
\hline \multirow{4}{*}{ 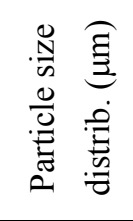 } & $\mathrm{D}_{10}$ & 29.9 & 29.1 & 29.0 & 30.9 & 29.6 & 31.5 & 4.20 & 4.13 \\
\hline & $\mathrm{D}_{50}$ & 61.1 & 59.7 & 61.4 & 60.9 & 59.1 & 59.7 & 54.6 & 52.0 \\
\hline & $\mathrm{D}_{90}$ & 101.9 & 97.6 & 104.7 & 99.4 & 98.5 & 97.3 & 174.9 & 167.5 \\
\hline & Span & 1.18 & 1.15 & 1.23 & 1.12 & 1.17 & 1.10 & 3.13 & 3.14 \\
\hline \multicolumn{2}{|c|}{$\begin{array}{c}\text { Specific surface area } \\
\left(\mathrm{m}^{2} / \mathrm{g}\right)\end{array}$} & 0.34 & 0.43 & 0.44 & 0.46 & 0.41 & 0.30 & 0.89 & 0.87 \\
\hline \multicolumn{2}{|c|}{ Poured density } & 663 & 610 & 647 & 648 & 634 & 655 & 596 & 590 \\
\hline \multicolumn{2}{|c|}{ Tapped density } & 802 & 786 & 771 & 778 & 774 & 817 & 875 & 874 \\
\hline \multicolumn{2}{|c|}{ Hausner ratio } & 1.21 & 1.29 & 1.19 & 1.20 & 1.22 & 1.25 & 1.47 & 1.48 \\
\hline \multicolumn{2}{|c|}{ Carr Index } & 17.3 & 22.4 & 16.1 & 16.7 & 18.1 & 19.8 & 31.9 & 32.5 \\
\hline \multicolumn{2}{|c|}{ Static angle of repose } & 26.6 & 30.0 & 30.6 & 29.1 & 29.7 & 28.9 & 46.6 & 44.9 \\
\hline \multicolumn{2}{|c|}{ Water (Karl Fischer) } & 5.16 & 4.52 & 5.00 & 4.50 & 4.87 & 4.82 & 4.58 & 5.06 \\
\hline \multicolumn{2}{|c|}{ Protein (ion selective) } & 272 & 188 & 93 & 136 & 199 & 225 & 124 & 79 \\
\hline
\end{tabular}


Table 2-2. The eight-staged ACI Aerodynamic Cut-off Diameter $\phi(\mu \mathrm{m})$ at Airflow Rate $Q=60 \mathrm{~L} / \mathrm{min}$ for DPI Experiment After Calibration.

\begin{tabular}{|c|c|c|c|c|c|c|c|c|c|}
\hline Stage & -1 & -0 & 1 & 2 & 3 & 4 & 5 & 6 & 7 \\
\hline Cutoff $\phi$ & 8.6 & 6.5 & 4.5 & 3.2 & 1.9 & 1.1 & 0.5 & 0.2 & Filter \\
\hline
\end{tabular}


Figure 2-1. The specification of the two inertial sampling devices used for aerosolization performance studies: (A) Twin stage liquid impinger (TSLI); (B) Andersen cascade impactor (ACI); (C) TSLI collection efficiency and semi-empirical oropharyngeal deposition curves (Model); (D) ACI collection efficiency curves (Adapted from Dunbar et al [33])

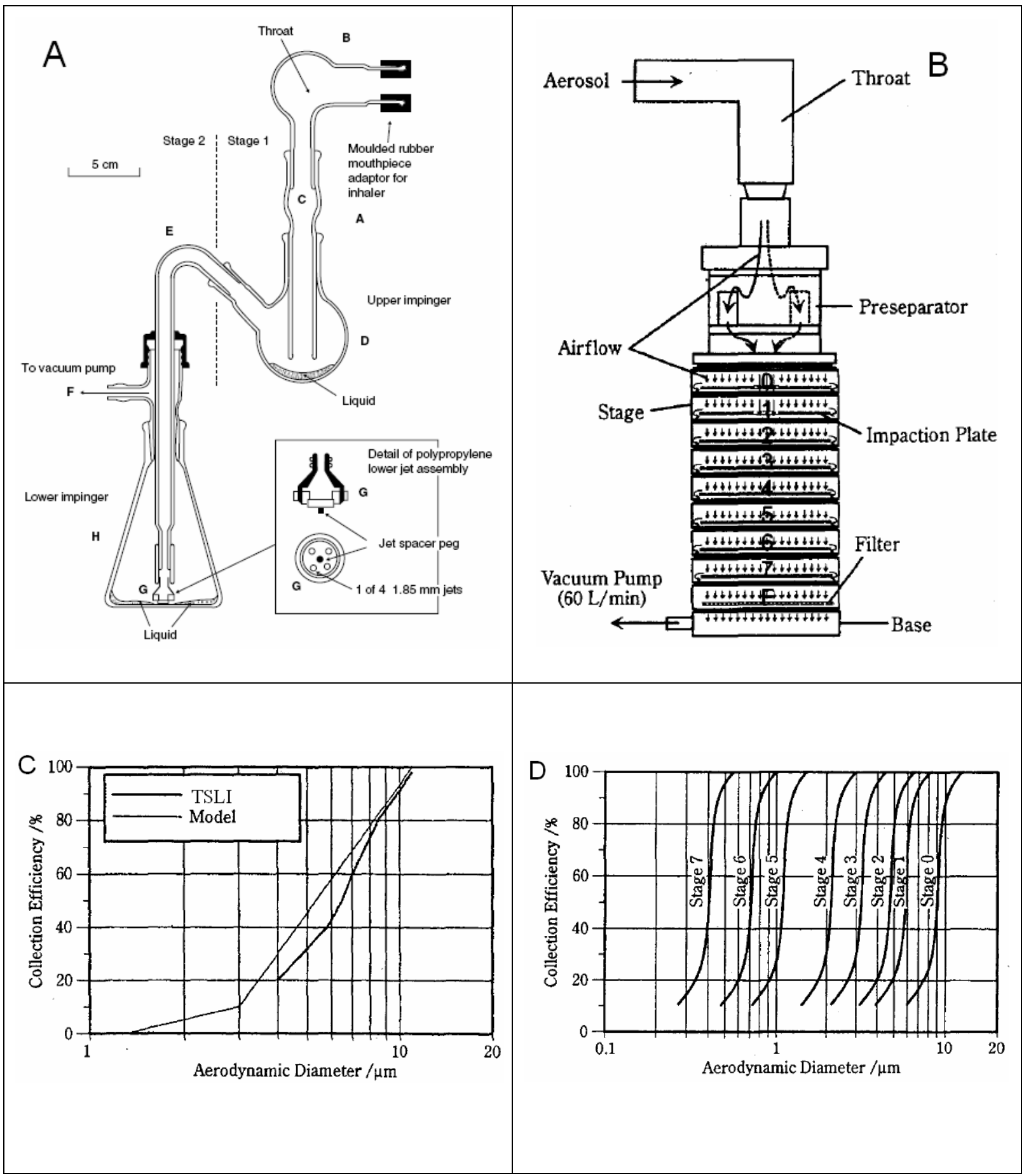


Figure 2-2. Representative scanning electron microscopy images of lactose monohydrate batches (A) SV-A; (B) SV-B; (C) SV-C; (D) SV-D; (E) SV-E; (F) SV-F; (G) ML-A; and (H) ML-B at $600 \times$ magnification.
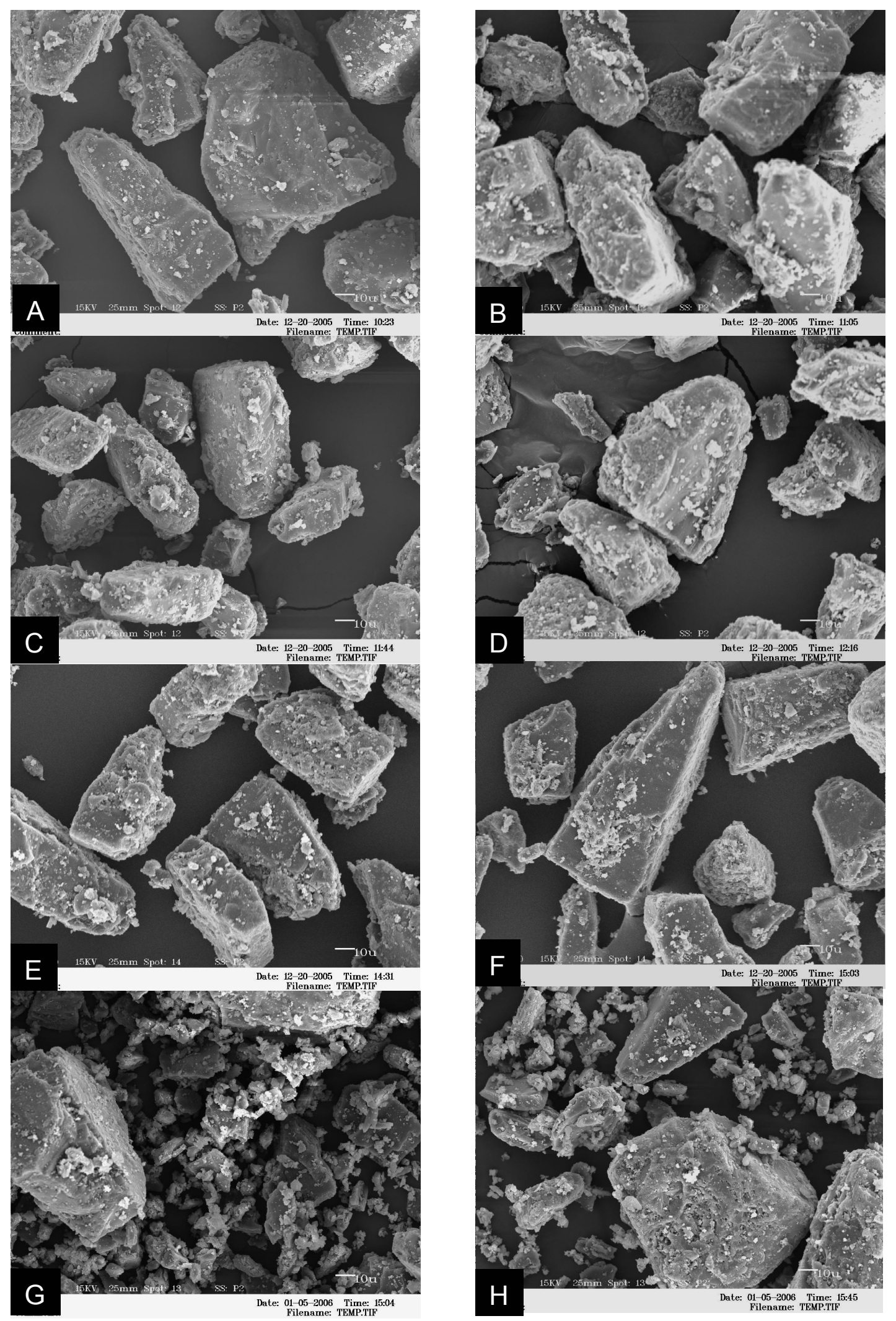
Figure 2-3. Representative scanning electron microscopy images of micronized albuterol sulfate (AS) (A) AS at 5,000× magnification and (B) AS at 20,000× magnification; lactose monohydrate-based blends (C) SV-B+0.5\%w/w AS; (D) ML-A+2\%w/w AS; (E) $\mathrm{SV}-\mathrm{B}+2 \% \mathrm{w} / \mathrm{w} \mathrm{AS} ;(\mathrm{F}) \mathrm{SV}-\mathrm{C}+2 \% \mathrm{w} / \mathrm{w}$ AS at $1,500 \times$ magnification.
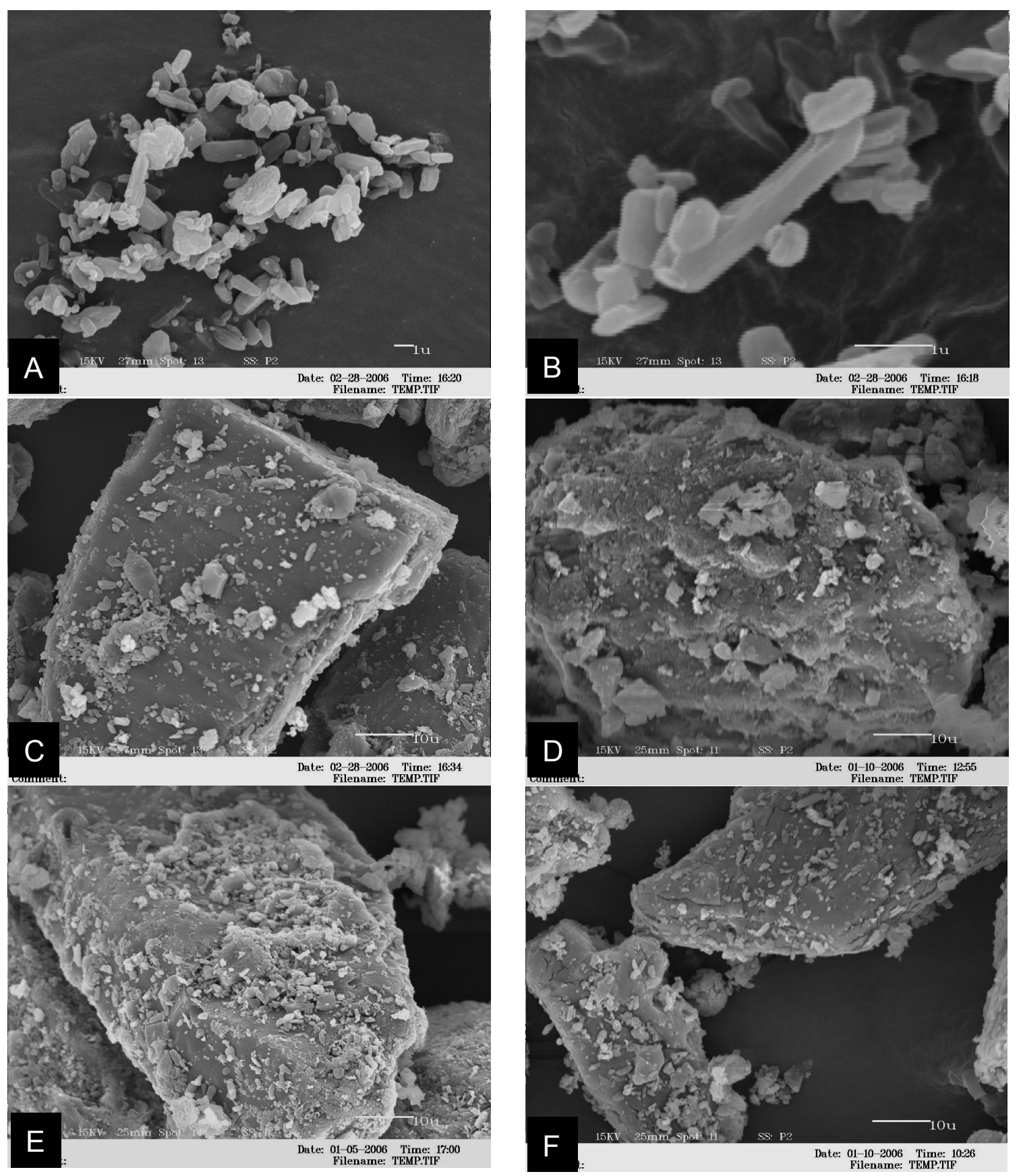
Figure 2-4. Representative differential scanning calorimetry thermograms of (A) SV-A and SV-B; (B) ML-A and ML-B; (C) and (D) micronized albuterol sulfate; (E) blends of AS $(2 \% \mathrm{w} / \mathrm{w})$ with SV-A and SV-B, respectively; (F) blends of AS $(2 \% \mathrm{w} / \mathrm{w})$ with ML-A and ML-B, respectively.
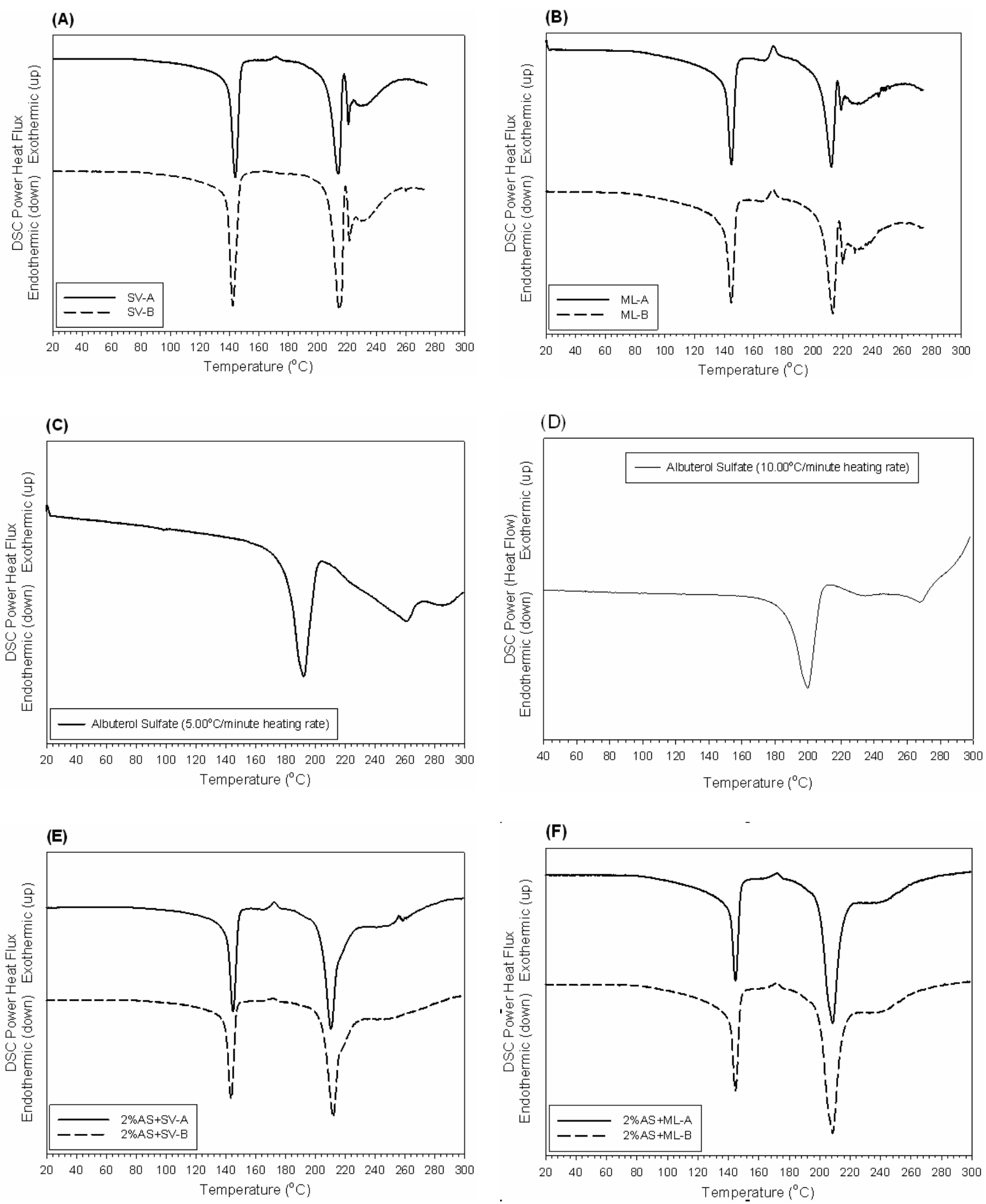
Figure 2-5. Representative X-ray powder height intensity diffractograms of lactose monohydrate (A) SV; (B) ML (Raw data: Courtesy of Dr. Mansour. The data were re-plotted using Match ${ }^{\circledR}$ software, Crystal Impact, Inc.); (C) the University of Cambridge database of pure $\alpha$-lactose monohydrate; (D) the University of Cambridge database of pure $\beta$-lactose.

\section{(A)}

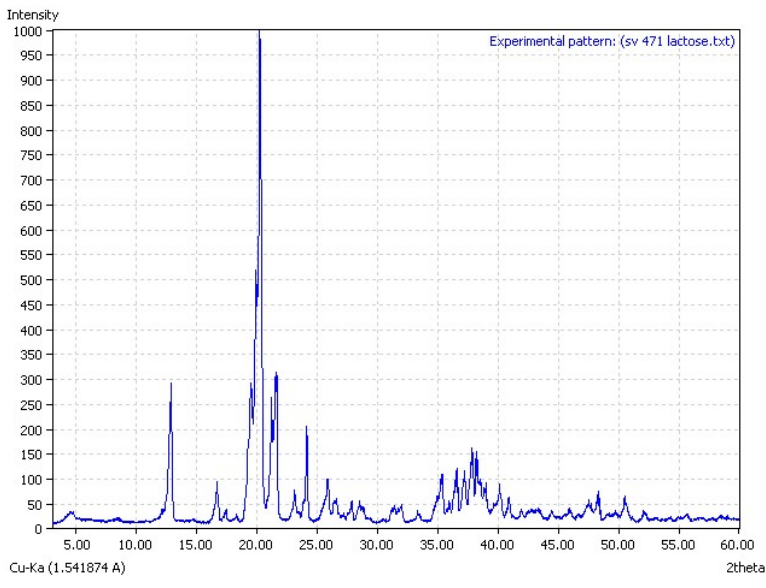

(C)

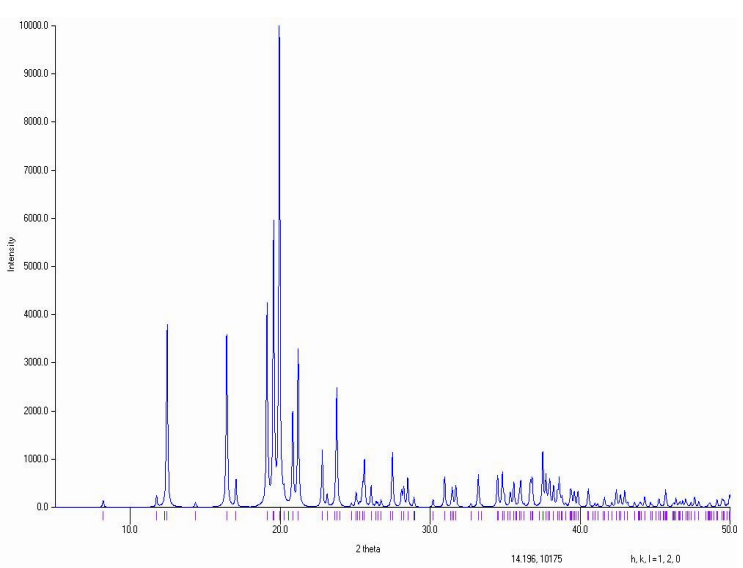

(B)

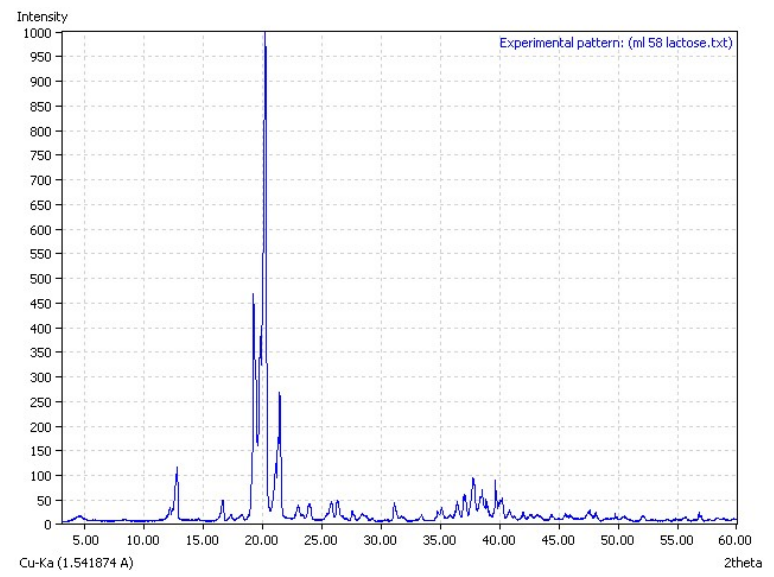

(D)

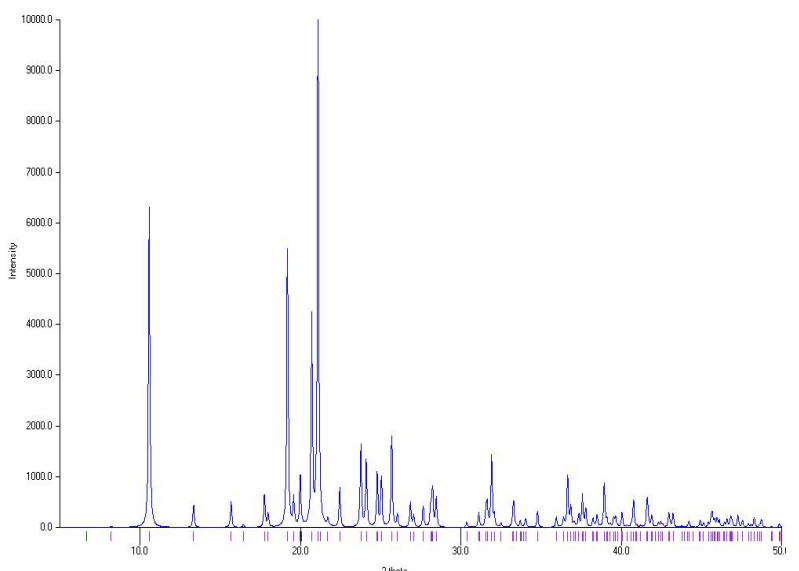


Figure 2-6. Representative atomic force microscopy topographic images of lactose monohydrate by tapping mode: (A) SV-B $(10 \mu \mathrm{m} \times 10 \mu \mathrm{m})$; (B) SV-C $(10 \mu \mathrm{m} \times 10 \mu \mathrm{m})$; (C) ML-B $(10 \mu \mathrm{m} \times 10 \mu \mathrm{m})$; (D) ML-B $(50 \mu \mathrm{m} \times 50 \mu \mathrm{m})$; and $(\mathrm{E}-\mathrm{F}) \mathrm{SV}-\mathrm{F}(5 \mu \mathrm{m} \times 5 \mu \mathrm{m})$

(A)

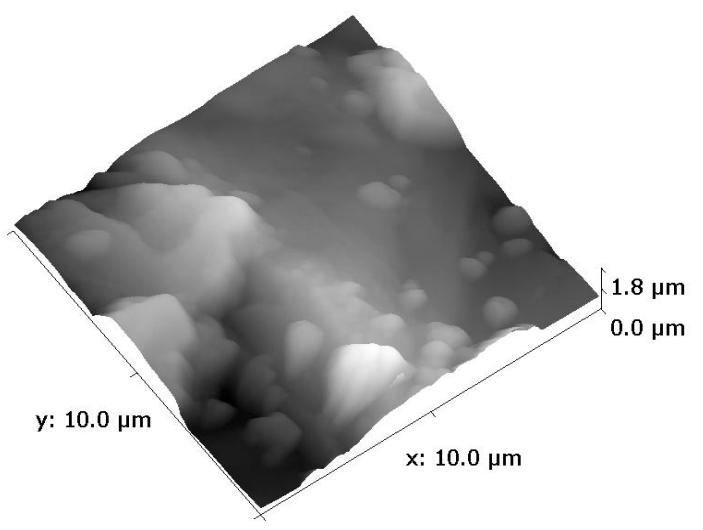

(C)

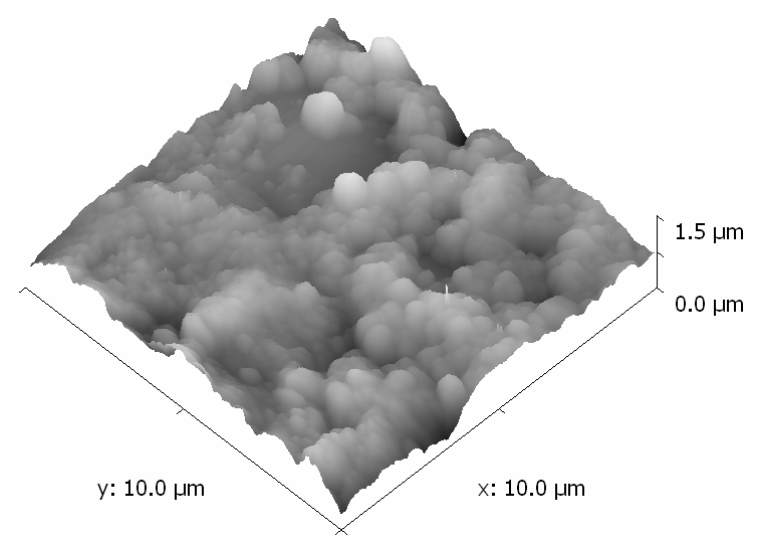

(E)

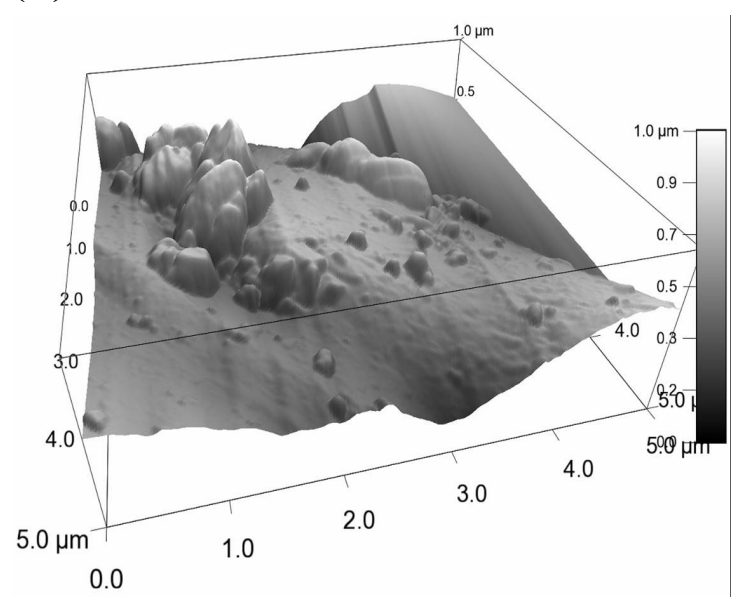

(B)

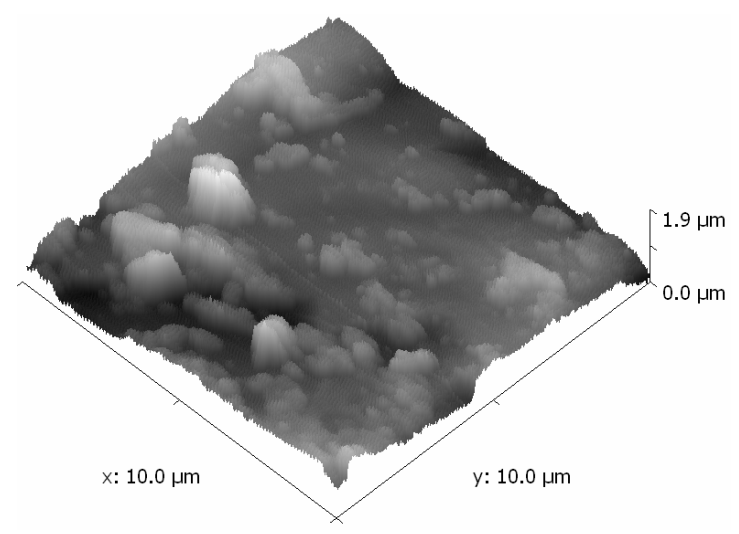

(D)

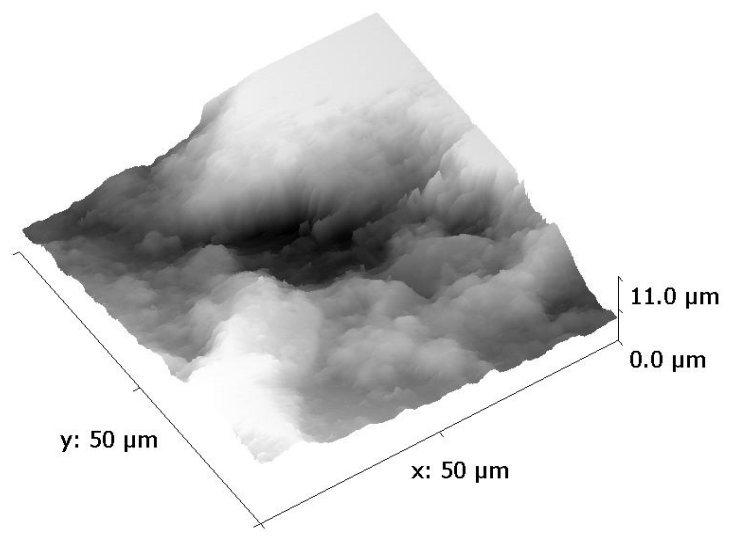

(F)

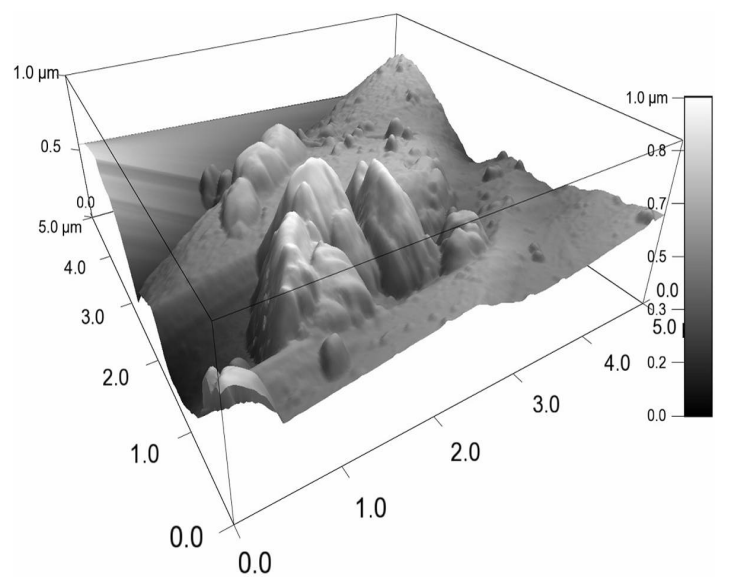


Figure 2-7. The results of TSLI performance study of intereactive physical mixtures: albuterol sulfate (AS) and lactose monohydrate (SV131 and ML058). Two concentrations of AS were prepared at $0.5 \% \mathrm{w} / \mathrm{w}$ and $2 \% \mathrm{w} / \mathrm{w}$. The performance results (ED and $\mathrm{FPF}_{\mathrm{TD}}$ ) were plotted against SET parameters (pressure drop, Reynolds number, and shear stress).

(A)

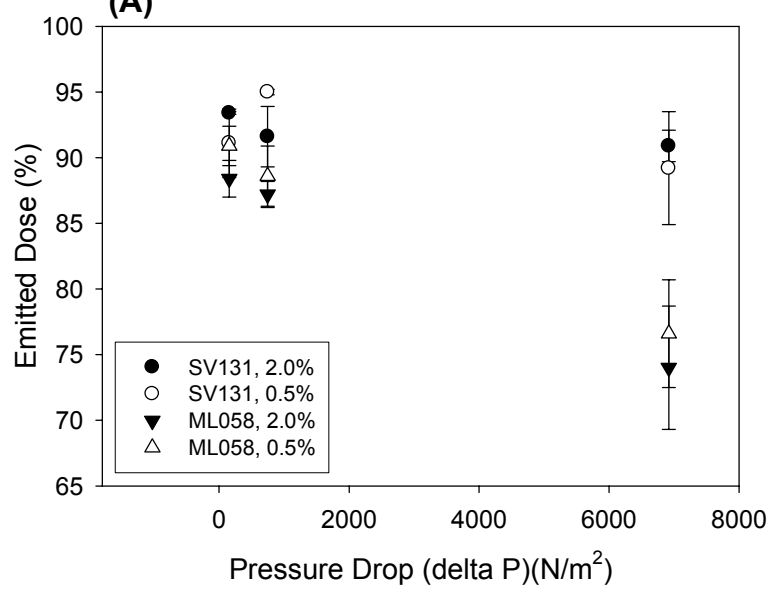

(B)

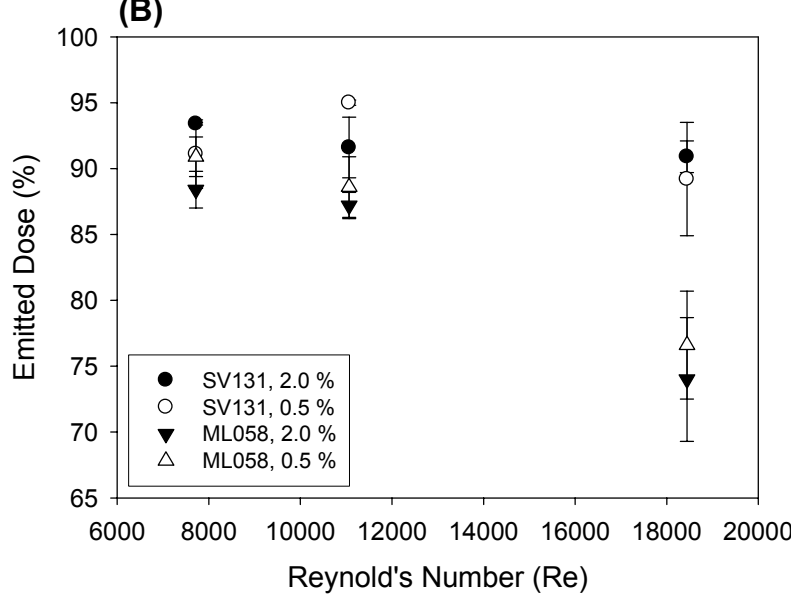

(C)

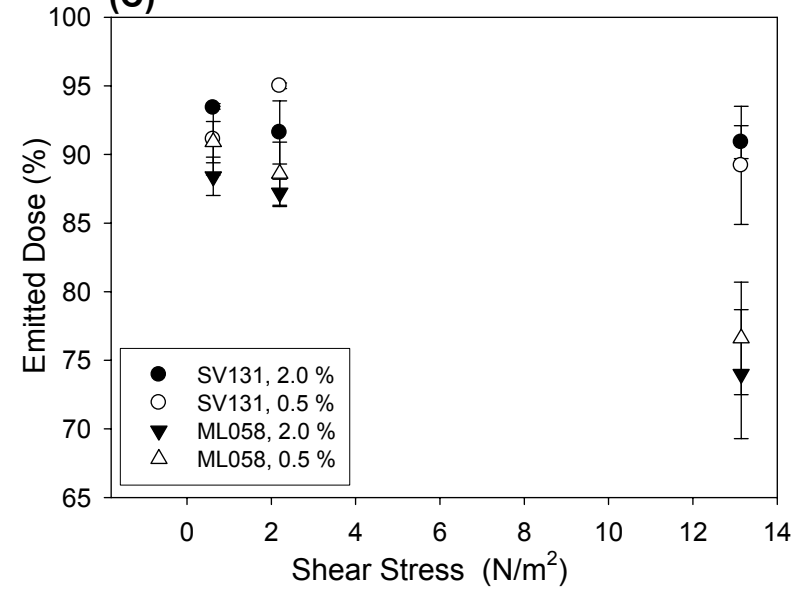

(D)

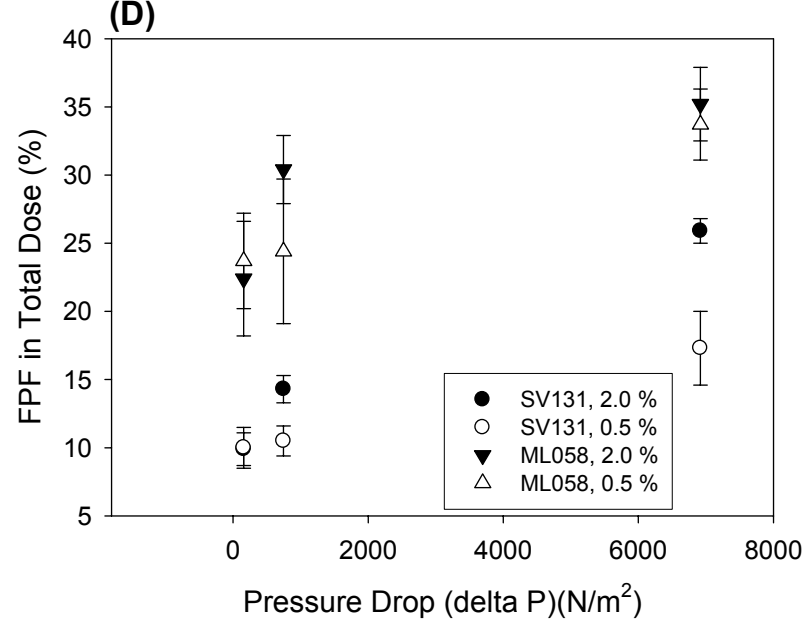

(E)
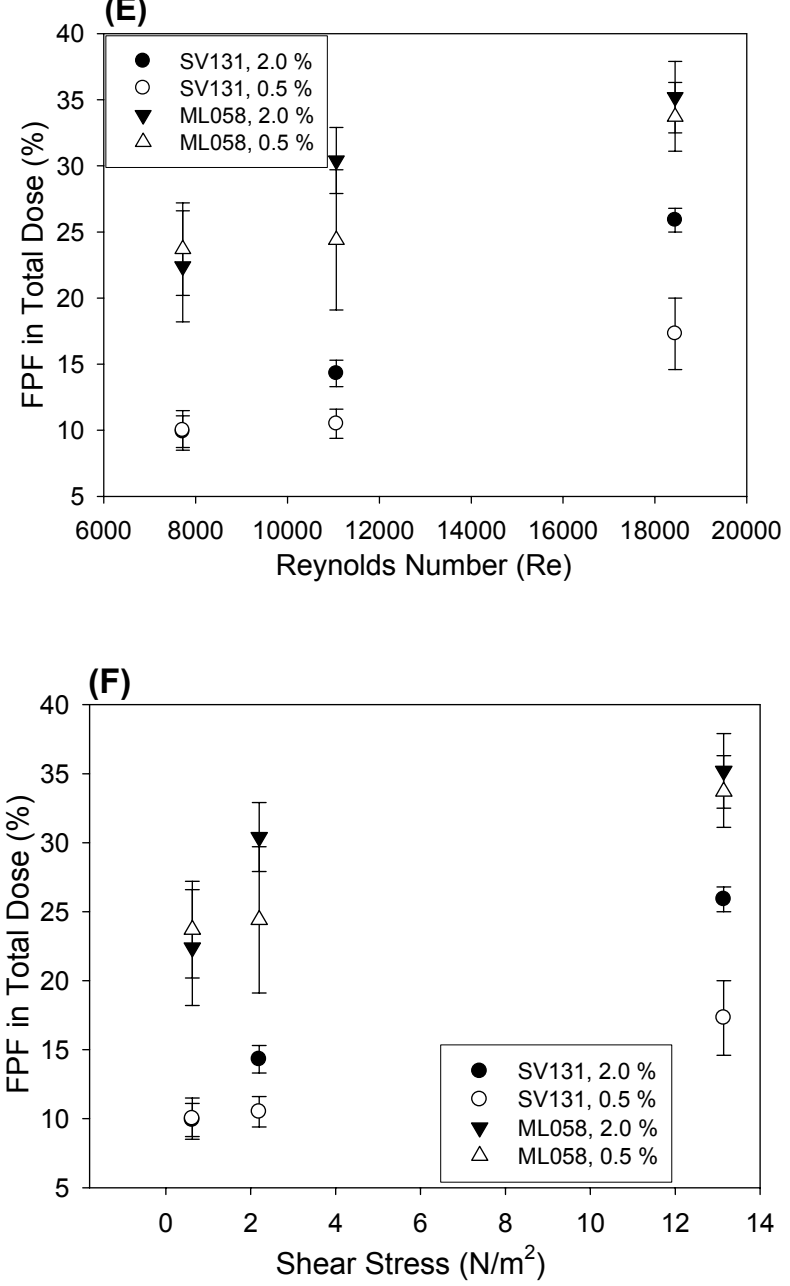
Figure 2-8. The results of ACI performance study of intereactive physical mixtures: albuterol sulfate (AS) and lactose monohydrate (SV131 and ML058): (A) ED of 0.5\%w/w AS-SV131; (B) ED of 2\%w/w AS-ML058; (C) FPF $_{\mathrm{TD}}$ of $0.5 \% \mathrm{w} / \mathrm{w}$ AS-SV131; (D) $\mathrm{FPF}_{\mathrm{TD}}$ of $2 \% \mathrm{w} / \mathrm{w}$ AS0-ML058; (E) MMAD when solenoid switch was operated off; (F) MMAD when solenoid switch on.

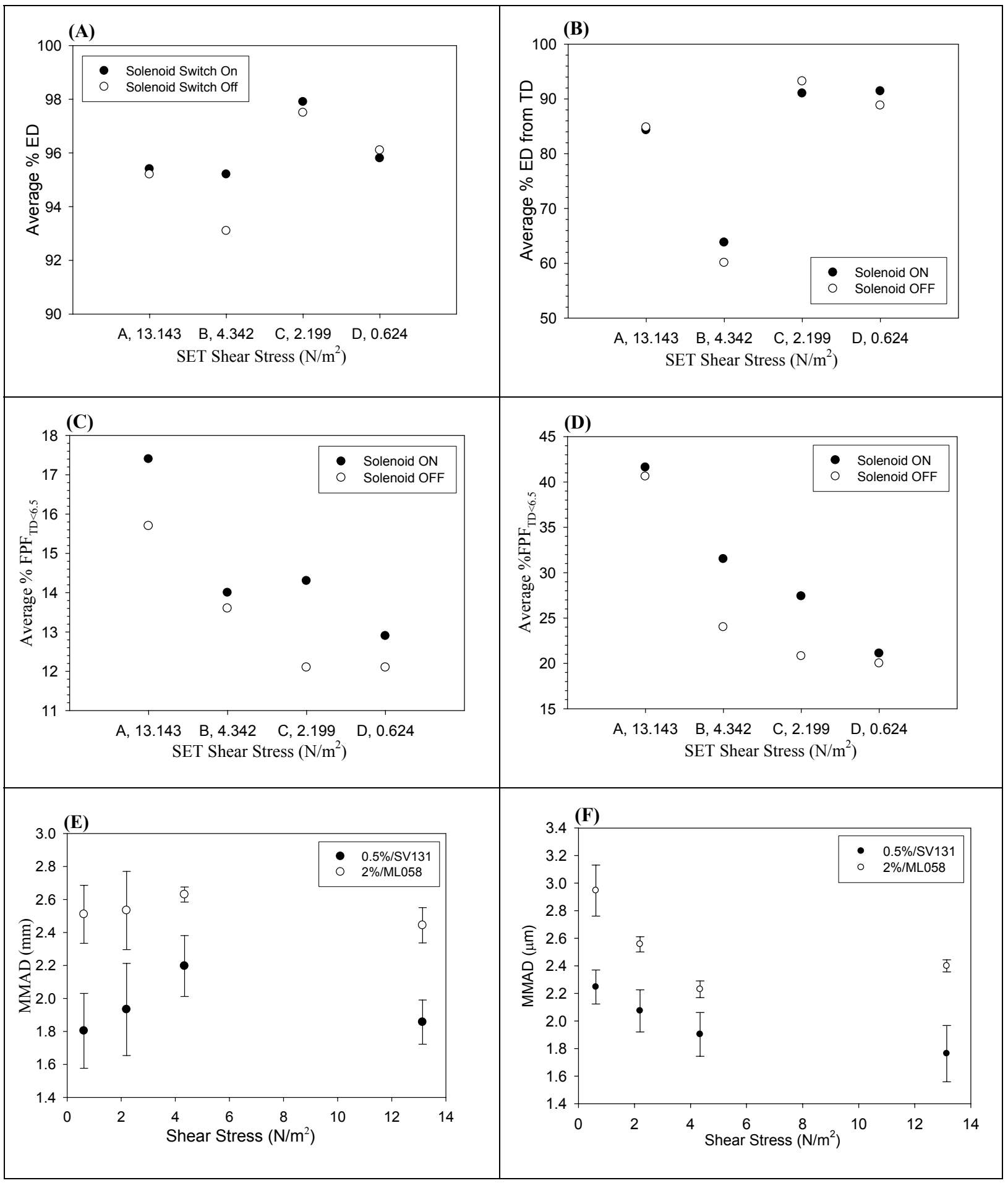




\section{References}

1. R.C. Rowe, P.J. Sheskey and S.C. Owen, in: Handbook of Pharmaceutical Excipients, R.C. Rowe, P.J. Sheskey, and S.C. Owen, Vol. 1, pp. 385-398. Pharmaceutical Press and American Pharmacists Association, Washington, DC (2006).

2. B.C. Hancock and S.L. Shamblin, Pharm Sci Technol Today, 1, 345-351 (1998).

3. H. Smyth and A.J. Hickey, Am J Drug Deliv, 3, 117-132 (2005).

4. J. Bronlund and T. Paterson, Int Dairy J, 14, 247-254 (2004).

5. K.D. Ross, J. Dairy Sci., 61, 152-158 (1978).

6. S.L. Raghavan, R.I. Ristic, D.B. Sheen and J.N. Sherwood, J Pharm Sci, 90, 823-32 (2001).

7. G. Clydesdale, K.J. Roberts, G.B. Telfer and D.J. Grant, J Pharm Sci, 86, 135-41 (1997).

8. S.K. Dwivedi and A.G. Mitchell, J Pharm Sci, 78, 1055-6 (1989).

9. K.D. Foster, J. Bronlund and T. Paterson, Int Dairy J, 15, 411-418 (2005).

10. R. Price, P.M. Young, S. Edge and J.N. Staniforth, Int J Pharm, 246, 47-59 (2002).

11. R.J. Lloyd, X.D. Chen and J.B. Hargreaves, Int J Food Sci Tech, 31, 305-311 (1996).

12. X.M. Zeng, G.P. Martin and C. Marriott, Particulate Interactions in Dry Powder Formulations for Inhalation. Taylor \& Francis, New York (2001).

13. D. Mahlin, J. Berggren, G. Alderborn and S. Engstrom, J Pharm Sci, 93, 29-37 (2004).

14. R. Price and P.M. Young, J Pharm Sci, 93, 155-64 (2004).

15. F. Thielmann, D.J. Burnett and J.Y. Heng, Drug Dev Ind Pharm, 33, 1240-1253 (2007).

16. H. Steckel, P. Markefka, H. teWierik and R. Kammelar, Int J Pharm, 309, 51-59 (2006).

17. F. Podczeck, Int J Pharm, 160, 119-130 (1998). 
18. J.N. Staniforth, in: Respiratory drug delivery, R. Dalby, P.R. Byron, and S.T. Farr, Vol. 5, pp. 65-73. Interpharm Press, Buffalo Grove, IL (1996).

19. A.J. Hickey, H.M. Mansour, M.J. Telko, Z. Xu, H.D. Smyth, T. Mulder, R. McLean, J. Langridge and D. Papadopoulos, J Pharm Sci, 96, 1282-1301 (2007).

20. Available from: http://en.wikipedia.org/wiki/Differential_scanning_calorimetry.

21. E. Pungor and G. Horvai, A Practical Guide to Instrumental Analysis: Differential Scanning Calorimetry. CRC Press, Boca Raton, FL (1994).

22. D. Giron, J Pharm Biomed Anal, 4, 755-70 (1986).

23. L.R. Hilden and K.R. Morris, J Pharm Sci, 93, 3-12 (2004).

24. B. Perrenot and G. Widmann, Thermochimica Acta, 234, 31-39 (1994).

25. C.L. Stevenson, D.B. Bennett and D. Lechuga-Ballesteros, J Pharm Sci, 94, 1861-80 (2005).

26. P.H. Hermans and A. Weidinger, J Appl Physics, 19, 491-506 (1948).

27. Powder diffraction file, inorganic phases, organic and organometallic phases search manual, http://www.icdd.com/

28. Suryanarayanan, in: Physical Characterization of Pharmaceutical Solids, H.G. Brittain (Eds.), Vol. pp. 187-221. Marcel Dekker, New York (1995).

29. E.R. Beach, G.W. Tormoen, J. Drelich and R. Han, J Colloid Interface Sci, 247, 84-99 (2002).

30. J.K. Eve, N. Patel, S.Y. Luk, S.J. Ebbens and C.J. Roberts, Int J Pharm, 238, 17-27 (2002).

31. Y.I. Rabinovich, J.J. Adler, A. Ata, R.K. Singh and B.M. Moudgil, J Colloid Interface Sci, 232, 17-24 (2000).

32. R.C. Anderson, D.R. Buchele, E.A. Hovenac and J.A. Lock, Laser diffraction particle sizing: instrument probe volume relocation and elongation, NASA Technical Memorandum. (1990).

33. C.A. Dunbar, A.J. Hickey and H. P., Kona, 16, 7-45 (1998). 
34. G.W. Hallworth and D.G. Westmoreland, J Pharm Pharmacol, 39, 966-72 (1987).

35. British Pharmacopoeia. Her Majesty's Stationary Office, London, UK (1998).

36. T.T. Mercer, Ann Occup Hyg, 6, 1-14 (1963).

37. C. Wang, in: Inhaled Particles, (Eds.), Vol. 1, pp. 31-54. Elsevier Ltd., London (2005).

38. A.J. Hickey, H.M. Mansour, M.J. Telko, Z. Xu, H.D. Smyth, T. Mulder, R. McLean, J. Langridge and D. Papadopoulos, J Pharm Sci, 96, 1302-1319 (2007).

39. M.D. Louey, M. VanOort and A.J. Hickey, J. Aerosol Sci., 37, 1520-1533 (2006).

40. A.R. Clark and A.M. Hollingsworth, J Aerosol Med, 6, 99-110 (1993).

41. W.C. Hinds, Aerosol Technology: Properties, Behavior, and Measurement of Airborne Particles. John Wiley \& Sons, New York (1999).

42. C.A. Dunbar, B. Morgan, M. Van Oort and A.J. Hickey, PDA J Pharm Sci Technol, 54, 478-484 (2000).

43. B.Y. Shekunov, J.C. Feeley, A.H. Chow, H.H. Tong and P. York, J Aerosol Sci, 34, 553-568 (2003).

44. H. Larhrib, G.P. Martin, C. Marriott and D. Prime, Int J Pharm, 257, 283-296 (2003).

45. J. Raula, F. Thielmann, J. Kansikas, S. Hietala, M. Annala, J. Seppala, A. Lahde and E.I. Kauppinen, Pharm Res, 25, 2250-2261 (2008).

46. K. brandenburg and H. Putz. [cited Jan, 2010; Available from: http://www.crystalimpact.com/match/Default.htm.

47. D. Traini, P.M. Young, F. Thielmann and M. Acharya, Drug Dev Ind Pharm, 34, 992-1001 (2008).

48. Z. Xu, H.M. Mansour, T. Mulder, R. McLean, J. Langridge and A.J. Hickey, Respiratory Drug Delivery, 3, 897-900 (2008). 


\section{CHAPTER 3 DRUG PERFORMANCE STUDY}

\subsection{Introduction}

The therapeutic implication of respiratory drug delivery for the treatment of asthma has been discussed in Chapter 1.2. The drugs involved for disease therapy, based on their pharmacological mechanism of action, are mainly classified into five types: 1) $\beta$-adrenergic agonists; 2) antichlolnergics; 3 ) corticosteroids; 4) cromone derivatives; and 5) xanthine derivatives. Among them, 1), 2), and 5) are called bronchodilators, but their bronchodilation mechanisms are different. The corticosteroids reduce or control inflammatory mediators. The cromone derivatives are a special class of drugs that prevent mast cell degranulation. In this chapter, the focus is on the physicochemical characterization of four model drugs and their interactive physical mixtures with four selected lactose monohydrate batches. The dry powder aerosols would be generated by standardized entrainment tubes (SETs) from drug blends with different batches of lactose monohydrate. The aerosolization performance was assessed by twin-stage liquid impinger (TSLI) and Andersen cascade impactor (ACI).

The objectives of this study were: discriminatory assessment of dry powder aerosol performance using SETs and lactose monohydrate-based formulations and development of a novel analytical tool for aerosolization performance evaluation and prediction: the powder aerosol deaggregation equation (PADE). A fold-over experimental design and statistical analyses were also included in parallel with the PADE method. 


\subsubsection{Drugs for the Treatment of Asthma and COPD}

Four model drugs: albuterol sulfate (AS), disodium cromoglycate (DSCG), ipratropium bromide monohydrate (IPB), and fluticasone propionate (FP) that represent four different classes of asthma and COPD treatment were selected (Figure 3-1).

The first drug, AS, is a short-acting $\beta_{2}$-adrenergic agonist with a long history of use for the relief of bronchospasm in asthma and COPD.[1, 2] The second drug, DSCG, having resulted in the first marketed dry powder inhalation aerosol product, is a mast cell stabilizer for the treatment of asthma whose origins are in anecdotal reports of natural product remedies. Its exact mechanism of action has yet to be elucidated.[3] The third drug, IPB induces bronchodilation and inhibits mucus secretion by competitive inhibition of muscarinic cholinergic receptors, causing blockade of acetylcholine-induced stimulation of guanyl cyclase, which, in turn, reduces the formation of cyclic guanosine monophosphate (cGMP), a mediator of bronchoconstriction.[4] It is a non-selective, short-acting bronchodilator (as the approved proprietary aerosol drug product, Atrovent ${ }^{\mathrm{TM}}$, Boehringer Ingelheim) for the treatment of bronchial spasms associated with chronic obstructive pulmonary disease, including chronic bronchitis and emphysema. It has also been co-administered (Combivent ${ }^{\mathrm{TM}}$, Boehringer Ingelheim) with $\beta_{2}$ adrenergic agonist albuterol sulfate through both anticholinergic and sympathomimetic mechanisms, which can achieve additive bronchodilator effect and prevent $\beta_{2}$ agonist induced bronchospasm (asthma paradox).[5] The fourth drug, FP, is a synthetic steroid of glucocorticoid family used as an anti-inflammatory agent. The anti-inflammatory actions of FP in asthma are believed to result from direct inhibition of multiple cell types involved in airway 
inflammation and inflammatory mediators produced by these cells.[6] On molecular level, FP not only suppresses immune response, but also inhibits prostaglandins and leukotrienes, as well as cyclooxygenases (both COX-1 and COX-2) expression, providing long-term control of persistent asthma (Flovent ${ }^{\mathrm{TM}}$, GlaxoSmithKline) and allergic rhinitis (Flonase $^{\mathrm{TM}}$, GlaxoSmithKline) symptons.[7]

\subsubsection{Physicochemical Properties of Drugs}

AS is an ionic salt with electropositive properties, and perhaps the most extensively studied drug in therapeutic aerosol formulation.[2] The solid-state properties of DSCG is unique in that it quickly and reversibly sorbs and liberates water to form nonstoichiometric hydrates with the change of crystal lattice parameters.[8] When crystalline DSCG hydrate molecule collapses at high relative humidity, it demonstrates liquid crystalline properties by forming lyotropic mesophases at $20{ }^{\circ} \mathrm{C}$.[9] IPB is a synthetic quaternary $\mathrm{N}$-methyl isopropyl derivative of noratropine. As a hydrated salt crystalline powder, it is freely soluble in water and lower alcohol, but insoluble in lipophilic solvents such as ether, chloroform, and fluorocarbons.[10] It is a highly hydrophilic drug $(\log P=-2.21)$.[11] A $1 \%$ aqueous solution of IPB has a $\mathrm{pH}$ of 5-7.5.[4] $\mathrm{FP}$, a fluorinated corticosteroid ester, is insoluble in aqueous buffer with a $\mathrm{pH}$ of 7.4.[12] It is a lipophilic drug $(\log P=3.46)$ passing readily through the cell membrane and binding with the corticosteroid receptor.[6] The true densities of IPB and FP are 1.33 and $1.37 \mathrm{~g} / \mathrm{cm}^{3}$, respectively. 
Micronized AS (Pfizer Global R\&D, Kent, UK) was used as supplied. DSCG (Sigma-Aldrich Inc., St. Louis, MO), IPB (Sigma-Aldrich Inc., St. Louis, MO), and FP (APAC Pharmaceuticals, Columbia, MD) were purchased in unmilled form and micronized using a Trost Impact Pulverizer (Gem T Research Model Jet Mill, Garlock Inc., Plastomer Products, Newton, PA). Lactose monohydrate (Respitose ${ }^{\mathrm{TM}}$ ) batches of two milled (designated as ML-A and ML-B) and two sieved batches (designated as SV-A and SV-B) were provided by DMV-Fonterra Excipients. (These four batches of lactose monohydrates were selected from initially 35 batches, then 8 batches (six sieved and two milled) of lactose monohydrates based on two principal components analyses,[13] and cluster analyses performed independently. These analyses were used to assess the diversity of batches with respect to characteristics such as particle size, bulk and tap density, water activity, etc. (see Table 2-1). The initial results showed drug concentration $(0.5$ and $2 \% \mathrm{w} / \mathrm{w})$ was the least significant factor compared with lactose carrier and SET shear stress (see Chapter 2). The rigorous aerosol performance evaluation required preliminary identification of parameters of interest. Consequently, as the least significant factor, drug concentration, was excluded (fixed at $2 \% \mathrm{w} / \mathrm{w}$ ), to allow greater focus on the drug, lactose monohydrate, and shear effects. However, concentration remains an important factor for future assessment.

\subsection{Methods}

\subsubsection{Analytical Approaches}




\subsubsection{Aerosol Formulation Preparation}

The Jet-milling of DSCG, IPB, and FP (2 g/each) was conducted at opposing inlet/outlet pressure of $60 / 40,75 / 55,75 / 55$ psig, respectively, using dry $\mathrm{N}_{2}$ gas (prepurified, Airgas National Welders Supply, Charlotte, NC) to achieve appropriate size and distribution. The yield of micronized particles collected was $\sim 50-70 \%$. The particle size distribution and morphology were examined by laser diffraction (LD) and scanning electron microscopy (SEM). The powder obtained was re-milled if the volume median diameter was larger than $5 \mu \mathrm{m}$ and re-characterized by LD and SEM.

The interactive physical mixtures $(2 \mathrm{~g}$ each, $2 \% \mathrm{w} / \mathrm{w}, 4$ drugs $\times 4$ carriers $=16$ blends) were prepared as follows: The micronized drugs were allowed to pass through a $125 \mu \mathrm{m}$ sieve to reduce the degree of agglomeration. A pre-blend of drugs (40 $\mathrm{mg}$ each) with lactose monohydrate batches (1960 mg each) was prepared by geometrical addition in an aluminum foil container using a spatula. The pre-blends were further processed in a Turbula ${ }^{\circledR}$ mixer (Model T2C, GlenMills Inc., Clifton, NJ) for 20 min. Blend uniformity was evaluated by analyzing 5 samples of each mixture taken from different regions of the blends. The amounts of AS, DSCG, IPB, and FP in the blends were quantified by UV absorption spectroscopy measured at a wavelength of $\lambda=225,223,211$, and $238 \mathrm{~nm}$ (Shimadzu UV-1700 UV/Vis spectrometer, Shimadzu Corporation, Columbia, Maryland), respectively, in deionized water (Barnstead Nanopure, 18.0 mega-ohm) except FP blends, which was dissolved in $\mathrm{EtOH} / \mathrm{H}_{2} \mathrm{O}$ mixture (ratio $=60: 40$ ). In all cases, a coefficient of variation $(\% \mathrm{CV})<5.0 \%$ was considered homogeneous. The prepared interactive physical mixtures were placed in tightly sealed glass jars (Wheaton Science Products, Millville, NJ) 
which were then stored in glass desiccators (Drierite ${ }^{\circledR} /$ Indicating Drierite ${ }^{\circledR}$ ) under ambient temperature and pressure prior to further characterization and aerosolization performance study.

\subsubsection{Scanning Electron Microscopy (SEM)}

The volume size, morphology, surface features and blend uniformity of drugs, lactose monohydrate batches and corresponding blends were examined by SEM. The samples were sputter-coated with Au-Pd in Argon for 90 s before the experiment (Polaron 5200 sputter coater, West Chester, PA). Scanning electron micrographs (Jeol JSM 6300V SEM, Peabody, NY) at a number of magnifications (x150, x600, x1.5k, x3k, x6k, x10k and $\mathrm{x} 20 \mathrm{k}$ ) were examined for both drugs before and after micronization, and at magnifications $(\mathrm{x} 60, \mathrm{x} 150, \mathrm{x} 600, \mathrm{x} 1.5 \mathrm{k})$ for all lactose monohydrate batches and drug/lactose monohydrate blends.

\subsubsection{Differential Scanning Calorimetry (DSC)}

The molecular and thermotropic phase transitions of each individual component including unmilled (original) drugs, milled drugs, lactose monohydrate batches, and interactive physical mixtures were examined by DSC (Perkin Elmer DSC 6 Workstation, Norwalk,

CT). Non-hermetically sealed aluminum pans containing 1.5-6.0 $\mathrm{mg}$ of samples were used. Nitrogen purge gas $\left(\mathrm{N}_{2}\right.$ prepurified, Airgas National Welders Supply, Charlotte, NC) was set at $20 \mathrm{~mL} / \mathrm{min}$. The temperature and heat flow were calibrated using high purity Indium (m.p. $=156.60{ }^{\circ} \mathrm{C}, \Delta \mathrm{H}_{\mathrm{f}}=28.45 \mathrm{~J} / \mathrm{g}$ ) and Zinc (m.p. $\left.=419.47{ }^{\circ} \mathrm{C}, \Delta \mathrm{H}_{\mathrm{f}}=108.37 \mathrm{~J} / \mathrm{g}\right)$ standards. Various scanning rates, including $2.0{ }^{\circ} \mathrm{C} / \mathrm{min}, 5.0{ }^{\circ} \mathrm{C} / \mathrm{min}$, and $10.0{ }^{\circ} \mathrm{C} / \mathrm{min}$, 
were applied to examine the thermodynamic phase behavior $\left(\mathrm{T}_{\mathrm{m}}\right)$ and kinetic/metastable phase transitions such as glass transitions $\left(\mathrm{T}_{\mathrm{g}}\right)$. For each sample, two separate scans were taken. The scanning range covered $20-300{ }^{\circ} \mathrm{C}$. Thermograms were processed and analyzed using the accompanying software (Pyris Thermal Analysis Instrument Control and Data Analysis Software, v.3.01).

\subsubsection{X-ray Powder Diffraction (XRPD)}

The degree of crystallinity and non-crystallinity of the drugs before and after milling, lactose monohydrate batches and interactive mixtures was examined by slit detector XRPD. (Regaku Multiflex, Tokyo, Japan). The powder was packed onto an etched square area of XRPD glass slide that was placed horizontally in the sample holder. Diffractograms were obtained to evaluate the crystallinity of the samples (1-2 g). The scanning was at $12 \theta$ degree/min and from 3-60 $2 \theta$ degree at room temperature $\left(23^{\circ} \mathrm{C}\right)$ to cover angles and nanometer distances where peaks occur for pharmaceutical material. The intensity of the peaks and the area under the curves were compared with the University of Cambridge XRPD database to estimate the long-range and short-range order of the material.

\subsubsection{Laser Diffraction (LD)}

The volume size distribution of drugs was characterized by Fraunhofer laser diffraction (Malvern Instruments 2600 Series, Worcs, UK) using micronized solid drug particles suspended in an organic dispersant (dispersant for fluticasone propionate was deionized water). The focal length of the lens is $63 \mathrm{~mm}$, giving a nominal size range of 0.5-118 $\mu \mathrm{m}$. 
The organic dispersant for the pulmonary drugs was chloroform, which is commonly used as a dispersing liquid in surface chemical and interfacial experiments due to its favorable combination of hydrophobicity and low viscosity. The solid-liquid dispersion was sonicated briefly $(20 \mathrm{~s})$ to eliminate any small aggregates while not physically breaking the primary particles (ie. particle fracture) and modifying the primary particle size. The LD data for lactose monohydrate batches were reported previously.

\subsubsection{In Vitro Aerosol Performance Characterization}

\subsubsection{Standardized Entrainment Tubes (SETs)}

Four SETs (denoted as SET-A, B, C, and D) with airflow parameters encompassing those of commercial DPI devices were selected. Their airflow parameters $\left(R e, \Delta P\right.$, power and $\left.\tau_{s}\right)$ at defined airflow rate were presented previously by Louey et al.[14] (see Table 1-1). The powder formulation ( $25 \mathrm{mg}$ /actuation) was transferred onto the loading area, which was at the proximal end of the inlet section, prior to actuation. The loading and SET setup was the same as described in Chapter 2. In order to evaluate the multiplicity of factors that influenced the aerosol formulation performance, the aerosolization was separated into two blocks. The first block of studies focused on two drugs: AS and DSCG; and the second block of studies focused on the other two: IPB and FP. The studies in each block were experimentally designed, the number of each experiment was randomly assigned (Design Expert 5.0.9 software, Stat-Ease Corp., MN), and performed at fixed airflow rate $(Q=60$ $\mathrm{L} / \mathrm{min}$ ). In vitro aerosolization was evaluated in triplicate under ambient condition. 


\subsubsection{Twin-staged Liquid Impinger (TSLI)}

The SETs were connected directly at the inlet port of the TSLI. A solenoid switch was placed between the TSLI and the aspirator to achieve instant actuation. A total of sixteen formulations (4 drugs $\times 4$ carriers) were assessed. After actuation $(25 \mathrm{mg}$ formulation/actuation, $10 \mathrm{sec}$ ), deposited drug (AS, DSCG, and IPB) fractions were collected by washing the SET and each portion of the TSLI with deionized water, then analyzed by UV-spectrometer $(\lambda=225,223$, and $211 \mathrm{~nm}$ for AS, DSCG, and IPB, respectively). For FP formulation, the solvent used for collecting deposited drug was $\mathrm{EtOH} / \mathrm{H}_{2} \mathrm{O}$ mixture $($ ratio $=60 / 40$ ), and analyzed by UV-spectrometer at $\lambda=238$. The aerodynamic behavior of these aerosol formulations was evaluated by emitted dose (ED: percentage of drug emitted from total dose), fine particle fraction (or absolute mass) from total dose $\left(F P F_{T D}\right.$ : percentage of mass (or absolute mass, $\left.F P M_{T D}\right)$ of drug below $6.4 \mu \mathrm{m}$ cut-off size from total dose), and fine particle fraction (or absolute mass) from emitted dose $\left(F P F_{E D}\right.$ : percentage of mass (or absolute mass, $\left.F P M_{E D}\right)$ of drug below $6.4 \mu \mathrm{m}$ cut-off size from emitted dose). In order to evaluate the multiplicity of factors including drugs, lactose monohydrate, and airflow conditions that influenced the formulation

performance, full factorial $\left(2^{3}\right)$ experimental analysis was designed, performed, and interpreted statistically (Design Expert 5.0.9 software, Stat-Ease Corp., MN). The pair-wise comparison (paired $t$-test) was performed using SigmaPlot 9.01. The performance data were also correlated with SET shear stress $\left(\tau_{s}\right)$ for further analysis.

\subsubsection{Andersen Cascade Impactor (ACI)}


The 8-stage ACI is an inertial impaction sizing device based on Stokes' terminal settling velocity and curvilinear particle motion, but is more discriminating and precise than the TSLI, offering several cut-off sizes for measuring aerosol particle dispersion and deposition.[15] The calibrated ACI aerodynamic cut-off diameters at an airflow-rate of 60 $\mathrm{L} / \mathrm{min}$ are listed in Table 2-2. The formulation performance was evaluated using the ACI (25 mg formulation/actuation $\times 2$ actuation, 10s). The mass median aerodynamic diameter (MMAD) for ACI was calculated as the $50^{\text {th }}$ percentile of the aerodynamic particle size distribution by mass, in addition to $E D, F P F$ and $F P M$. The $F P F_{6.5}$ (or $F P M_{6.5}$ ) and $F P F_{4.5}$ (or $F P M_{4.5}$ ) are fine particle fractions (or fine particle mass) below $6.5 \mu \mathrm{m}$ (stage 1 and under) and $4.5 \mu \mathrm{m}$ (stage 2 and under) cutoff diameter, respectively. Similar experimental analyses (but reduced sample number) were performed as those applied in TSLI study.

\subsection{Results}

\subsubsection{Aerosol Characterization}

\subsubsection{Particle size and morphology (SEM)}

Representative SEM images of micronized DSCG, IPB, and FP are shown in Figure 3-2.

The micronized AS, lactose monohydrate batches of ML and SV were shown in Figure 2-2 and 2-3, respectively. The interactive physical mixtures are shown in Figure 3-3. As expected, the images are consistent with the particle size analysis. All four drugs were in the respirable size range and similar narrow size distribution (Figure $\mathbf{2 - 3 A}$ and $\mathbf{B}, \mathbf{3 - 2 B}$, 
2G, and 2H) after air jet-milling. AS had relatively smooth surface textures with rectangular-shaped non-spherical particles. DSCG were more irregularly shaped and surface defects were observed at high magnifications. IPB particles exhibited regular morphology with relatively smooth surface after micronization, presumably because of the rigid crystalline monohydrate structure of its ionic salt. FP particles were more irregularly shaped with many superfine particles $(<1 \mu \mathrm{m})$ observed on the surface of the primary particles and independently by SEM. This result was not reflected in the particle size analysis, probably due to the lower detection limit of approximately $0.5 \mu \mathrm{m}$ of LD when using $63 \mathrm{~mm}$ focal lens. Particle agglomeration of these drugs was observed at all magnifications before blending, due to the high specific surface area of micronized particles and strong cohesive forces at the solid-solid interface. The SV lactose monohydrates were uniform particle shape, smooth, and had much fewer intrinsic fine particles (designated as particle size less than $10 \mu \mathrm{m}$ ) than ML lactose monohydrates, although the major particle size of both SV and ML batches were comparable (see Chapter 2). This was also consistent with the LD analyses. More fine particle agglomerates in ML lactose monohydrates were observed to spread between the void instead of adhering on the surface of major particles. It is hard to distinguish the fine lactose particles with the drug particles simply from the shape and morphology, especially milled lactose monohydrate containing many lactose fines. But there appeared to be an increase in particles in size range of $1-10 \mu \mathrm{m}$.

In AS blends, the increase in fine particles appeared to adhere more onto the major carrier particle surfaces, with more roughness or crevices, indicating "high surface 
energy" in these sites (Figure 2-3D, E, F). However, in DSCG blends, the increase in fine particles was observed to somehow detach from the major particle surfaces, and was more obvious in DSCG-ML systems which had more intrinsic fines (Figure 3-3A and B). Similarly, at $2 \%(\mathrm{w} / \mathrm{w})$ concentration, fine particles (both lactose monohydrate fines and drugs) were discretely distributed on the primary particles for IPB-SV and FP-SV blends, and more fine particles were observed on the rough surface regions of the primary lactose monohydrate particles. For the IPB-ML and FP-ML systems, more agglomerates of fines were observed to spread in the voids among the primary particles.

\subsubsection{Thermal properties (DSC)}

Representative DSC thermograms of DSCG, IPB and FP are shown in Figure 3-4. The thermograms of AS was shown in Figure 2-4. The thermograms of unmilled and micronized DSCG (Figure 3-4A) exhibited a pre-transition peak at about $90{ }^{\circ} \mathrm{C}$ and loss of water molecules occurred at about $100{ }^{\circ} \mathrm{C}$, which has often been observed in self-assembling surfactant system as a broad peak. It was reported that DSCG can accommodate $9 \mathrm{H}_{2} \mathrm{O}$ molecules in its crystal structure before collapsing into a liquid crystal. $[8,9]$ A slow scanning rate $\left(\leq 5^{\circ} \mathrm{C} / \mathrm{min}\right)$ was required to obtain meaningful thermotropic phase information and accurate $T_{m}$ measurements. Both unmilled and micronized DSCG had $T_{m}$ about $258{ }^{\circ} \mathrm{C}$, indicating highly distorted and stressed crystal forms.[9] However, the $T_{m}$ became undetectable when the scanning rate was reduced to 2 ${ }^{\circ} \mathrm{C} / \mathrm{min}$, possibly because of decomposition just above $T_{m}$. Unmilled DSCG had higher $\Delta H$ for both endothermic peaks $\left(\Delta H_{1}=93.3 \mathrm{~J} / \mathrm{g}, \Delta H_{2}=70.7 \mathrm{~J} / \mathrm{g}\right)$ than micronized sample 
$\left(\Delta H_{1}=75.1 \mathrm{~J} / \mathrm{g}, \Delta H_{2}=31.1 \mathrm{~J} / \mathrm{g}\right)$ in the same heating rate, indicative of partially loss of bound water and greater molecular disorder of the micronized sample due to milling. The thermograms of unmilled and micronized IPB and FP are shown in Figure 3-4B and C. The unmilled IPB plot has two endothermic peaks at $T_{1}=113{ }^{\circ} \mathrm{C}$ and $T_{2}=231{ }^{\circ} \mathrm{C}$ indicating the bound water removal $\left(\Delta H_{l}=129.8 \mathrm{~J} / \mathrm{g}\right)$ from the crystalline lattice and the melting of the crystalline anhydrate $\left(\Delta H_{2}=290.6 \mathrm{~J} / \mathrm{g}\right)$ at higher temperature. The micronized IPB exhibited endothermic peaks but reduced enthalpy $\left(T_{l}=109{ }^{\circ} \mathrm{C}, \Delta H_{l}=\right.$ 100.3 J/g; and $T_{2}=235^{\circ} \mathrm{C}, \Delta H_{2}=191.0 \mathrm{~J} / \mathrm{g}$ ) indicating partial loss of bound water from the monohydrate crystalline and increased molecular disorder from rigid crystalline during micronization. Some anhydrous IPB may be generated during milling. No crystalline polymorphic form was detected, before or after micronization. The thermograms of untreated and micronized FP were similar and did not show any distinctive peaks in the range of $20-270{ }^{\circ} \mathrm{C}$ at scanning rates of 2,5 , and $10{ }^{\circ} \mathrm{C} / \mathrm{min}$. However, peaks attributed to decomposition were observed in the range of $270-300{ }^{\circ} \mathrm{C}$. No DSC phase transition signal other than decomposition was observed. This is consistent with previous reports of both micronized and spray dried FP.[16, 17]

The thermotropic behavior of lactose monohydrate and DSCG, IPB, and FP blends are shown in Figure 3-5. AS blends was discussed previously in Chapter 2. For the DSCG and lactose monohydrate blends, the drug transitions were observed with broadening of the first endothermic peak of lactose monohydrates (indicated by the earlier onset temperature). Liquid crystalline effect of DSCG affected the transition of both SV and ML lactose monohydrate. The enhancement of the exothermic peak was distinct for 
both DSCG/ML blends ( $\Delta H$ of ML-A: $-10.8 \mathrm{~J} / \mathrm{g}$; ML-B: $-11.6 \mathrm{~J} / \mathrm{g}$ ) at $5{ }^{\circ} \mathrm{C} / \mathrm{min}$ scanning rate, while the exothermic peak was unnoticeable for both DSCG/SV blends. When SV or ML lactose monohydrate was blended with micronized IPB, appreciable peak broadening occurred, particularly for the second endothermic peak. A trace of the IPB melting peak was visible for some blends (ML-A) due to the large enthalpy. The exothermic peak intensity from lactose monohydrate did not increase after blending, and was observable only in ML lactose monohydrate blends. When SV or ML lactose monohydrate was blended with micronized FP, there was a slightly lower onset temperature of both endothermic peaks. An exothermic peak was observed for ML blends but not SV blends.

\subsubsection{Crystallinity (XRPD)}

XRPD graphs of micronized drugs (AS, DSCG, IPB, and FP) are shown in Figure 3-6. The XRPD \%Intensity diffractogram of micronized AS showed almost identical peak intensity when it was compared with the XRPD from the University of Cambridge database. It indicated that non-crystallinity mechanically induced by micronization was below the detection limit of XRPD diffractogram for AS. The XRPD \%Intensity diffractogram of DSCG indicated the presence of partially ordered and disordered liquid crystalline mesophase, which was characteristic of liquid crystalline hydrate materials. The degree of molecular order and self-assembly depended on the amount of bound water and temperature, which could also affect the solid state stability and macroscopic properties of aerosol performance. However, no crystalline polymorph of DSCG was observable, consistent with previous reports.[8,9] The \%Intensity of diffractogram of micronized IPB, when compared with published resources,[18] indicated a majority of 
crystalline material. The diffractogram of micronized FP was comparable to the previous reports of crystalline FP.[16, 19] Only polymorph I of FP was observed, whereas the metastable polymorph II, which could be formed in the supercritical fluid condition, was not detected in the entire scanning range. The diffractograms of SV and ML lactose monohydrate were shown previously in Figure 2-5.

\subsubsection{Particle size distribution (LD)}

The particle volume size distribution of jet-milled AS, DSCG, IPB, and FP before and after air jet-milling is shown in Table 3-1. AS and DSCG exhibited similar volume median diameters in the fine particle range $\left(D_{50}=3.59\right.$ and $3.68 \mu \mathrm{m}$, respectively) with narrow unimodal distribution ( $\operatorname{span}=0.26$ and 0.32 , respectively). IPB after first milling process did not yield ideal volume median diameter $\left(D_{50}=4.53 \mu \mathrm{m}\right)$ comparable to AS and DSCG, but the value of span was excellent (span =0.23). After the second milling, the particle size distribution was $D_{50}=3.29 \mu \mathrm{m}$, span $=0.34$. The FP was also subjected to milling twice because the poor $\mathrm{D}_{50}$ for the first milling process. The particle size distribution was $D_{50}=4.47 \mu \mathrm{m}$, span $=0.36$, which was comparable to the other three micronized drugs. The aerodynamic diameters (calculated from $D_{50}$ ) of IPB and FP were 3.79 and 5.23 , respectively.

The particle size and size distribution, morphology, thermal properties, and crystallinity of drug and carrier particles were characterized for consideration with respect to their influence on drug aerosolization performance. The influences of these physicochemical properties of both drugs and lactose monohydrates following interactive 
physical blending reflected the molecular properties of drugs and their effect on the drugs and carriers. These changes could also affect the macroscopic aerosol performance. The focus of the present work is on the influence of three major factors: drug, carrier lactose monohydrate, and SET shear stress to the aerosolization performance efficiency. Other factors including size, size distribution of both drug and lactose monohydrate particles, drug concentration, dose, interactive physical blending, and environment factors were, to a large extent, kept constant or comparable.

\subsubsection{In Vitro Aerosol Performance Assessment}

\subsubsection{TSLI}

The drug aerosolization performance of lactose monohydrate-based dry powder formulation is influenced by a variety of factors including physicochemical properties and surface features of given drugs and carrier particles, the parameters of airflow entrainment, and others.[20] Performance data obtained from TSLI studies are shown in Appendix 3-A1 and A2. For clarity, the influence of three factors: drug, lactose monohydrate, and SET shear stress on the aerosol performance will be discussed in categorical manner, then the overall effect will be summarized. The comparison of AS vs. DSCG will be discussed first, followed by IPB vs. FP. The unique assessment of aerosol performance using PADE will be applied to interpret the TSLI aerosol performance data.

Drug Effect. The DSCG formulations resulted in similar ED delivery to AS formulations, but superior FPF $(p<0.001)$. This trend of higher FPF was more remarkable at higher 
shear stress $(p<0.001)$. The FPF of AS formulations was more susceptible to carrier change than that of DSCG formulations.

The IPB and FP formulations gave similar ED at highest shear stress $(p>0.05)$. However, IPB formulations gave superior $E D$ values across all shear stress to FP formulations $(p<0.05)$. Compared with FP formulations, all IPB formulations gave superior $F P F$ values across all shear stress $(p<0.001)$. The FPF of IPB formulations was less susceptible to shear stress change than FP formulations and reached a plateau at relatively lower shear stress (see $F P F$ and shear stress correlation below for more explanation).

Carrier Effect (SV vs. ML). In AS formulations, the ED was significantly lower $(p<$ 0.001), but FPF $(p<0.001)$ was significantly higher for ML blends than SV blends. SV-B had slightly higher ED than SV-A $(p>0.05)$, but FPF was similar $(p>0.05)$. The ED for both ML blends was insignificant $(p>0.05)$, but FPF of ML-A was significantly higher than that of ML-B $(p<0.05)$. In DSCG formulations, the ED was significantly higher for SV blends than ML blends $(p<0.001)$, except at highest shear, where ED was less dependent on formulation effect. The FPF for SV-A was significantly lower than that of SV-B $(p<0.01)$; and ML-A was significantly lower than that of ML-B $(p<0.01)$. In all DSCG formulations, the ML lactose monohydrate batches gave superior aerosolization performance efficiency (higher FPF, $p<0.05$ ) to SV batches.

In both IPB and FP formulations, SV blends gave higher ED values than ML blends across all shear stress range $(p<0.001)$. However, at the highest shear $\left(\tau_{s}=13.143\right.$ 
$\mathrm{N} / \mathrm{m}^{2}$ ), ED exhibited the greatest independence of the formulation effect $(p>0.05)$. ML blends reached lowest ED value at shear stress produced by SET-B $\left(\tau_{s}=4.342 \mathrm{~N} / \mathrm{m}^{2}\right)$, then increased at the highest shear stress for both IPB and FP formulations. This could be explained by the effect of the SET design. SET-C and D have gradual enlargement and centrally located dose platform for maximizing flow, whereas SET-A and B have sudden enlargement and direct dose near the wall for maximizing shear.[14] However, SV blends showed a decreasing trend as shear stress was increased until ED values became relatively stable at high shear. SV-A gave superior ED values to that of SV-B $(p<0.05)$, whereas ML-A and B gave similar ED values $(p>0.05)$, but ML-A blend gave lower ED values than ML-B in FP formulation $(p<0.05)$. The FP with ML-A formulation showed an exceptional low ED value $(51.5 \%$, mean value, $n=3)$ at shear produced by SET-B. In both IPB and FP formulations, the ML blends gave superior performance efficiency (higher FPF values) to SV blends $(p<0.05)$. The FPF values in IPB blends showed less carrier type (ML or SV) influence $(p<0.05)$ than those in FP formulations $(p<0.001)$, especially at high shear stress range $\left(4.342-13.143 \mathrm{~N} / \mathrm{m}^{2}\right)$. In IPB formulations, the FPF value of SV-A was significantly higher than that of SV-B $(p<0.05)$, but the FPF values of ML formulations were similar $(p<0.05)$. In FP formulations, the FPF value of ML-B was significantly higher than that of ML-A $(p<0.001)$, but the FPF values of SV formulations were similar $(p>0.05)$.

SET Effect (Higher $\tau_{s}$ vs. Low $\left.\tau_{s}\right)$. When SET shear stress $\left(\tau_{s}\right)$ was correlated with aerosol performance $\left(\mathrm{ED}, \mathrm{FPF}_{\mathrm{TD}}\right.$, and $\left.\mathrm{FPF}_{\mathrm{ED}}\right)$, there was a general trend that the lowest shear stress $\operatorname{SET}\left(\tau_{s}=0.624 \mathrm{~N} / \mathrm{m}^{2}\right)$ gave higher ED (ED for AS: 83.4-93.1\%; ED for 
DSCG: 84.1-91.2\%; ED for IPB: 84.7-90.6\%; ED for FP: 84.0-90.5\%, mean value, $n=3$ ), but lower FPF $\left(\mathrm{FPF}_{\mathrm{TD}}\right.$ for AS: 6.8-21.4\%; $\mathrm{FPF}_{\mathrm{TD}}$ for DSCG: 20.9-25.4\%; $\mathrm{FPF}_{\mathrm{TD}}$ for IPB: 22.5-30.6\%; $\mathrm{FPF}_{\mathrm{TD}}$ for $\mathrm{FP}: 10.8-22.0 \%$, mean value, $n=3$ ) for all studied systems. In contrast, the highest shear stress SET $\left(\tau_{s}=13.143 \mathrm{~N} / \mathrm{m}^{2}\right)$ gave a relatively lower ED (ED for AS: 79.8-85.9\%; ED for DSCG: 74.8-81.0\%; ED for IPB: $67.7-71.2 \%$; ED for FP: 65.7-70.2\%, mean value, $\mathrm{n}=3$ ) but a dramatic higher $\mathrm{FPF}\left(\mathrm{FPF}_{\mathrm{TD}}\right.$ for $\mathrm{AS}: 14.7-42.9 \%$; $\mathrm{FPF}_{\mathrm{TD}}$ for DSCG: $33.6-43.3 \%$; $\mathrm{FPF}_{\mathrm{TD}}$ for IPB: $28.9-36.3 \%$; $\mathrm{FPF}_{\mathrm{TD}}$ for $\mathrm{FP}: 15.1-24.2 \%$, mean value, $n=3$ ). The difference of ED at highest shear for different blends was smaller than that at lower shear $\left(\tau_{s}=4.342,2.199\right.$ and $\left.0.624 \mathrm{~N} / \mathrm{m}^{2}\right)$, indicating less formulation dependency at the highest shear. A hyperbolic relationship was observed between FPF-shear stress correlations.

Overall Effect. In order to elucidate the complete set of factorial effects and interactions (drugs, lactose monohydrates, and SET shear stress) with respect to the aerosol performance $\left(\mathrm{ED}, \mathrm{FPF}_{\mathrm{TD}}, \mathrm{FPF}_{\mathrm{ED}}\right)$, full factorial analysis $\left(2^{3}\right)$ was applied (Appendix 3-B). The half-normal plots indicated that data fell on a straight line where random variation around a mean (normal distribution) was observed. The points that did not fit on the straight line indicated effects that could not be explained as chance occurrences, and were, therefore, considered significant. Cube plots were also included to compare the predicted (or actual) effects from coded levels of three factors.

The AS vs. DSCG formulations (blended with $2 \mathrm{SV}$ and $2 \mathrm{ML}$, respectively) were compared first. The result showed that the greatest contributing factor, SET shear stress, was significant in all comparisons. When SV lactose monohydrate were compared with 
ML lactose monohydrate, all three factors (drugs, carriers, and shear stress) were contributing variables and there were drug/carrier interaction and carrier/shear stress interaction. No carrier effect was observed when SV-A and SV-B were compared. Neither was ML-A and ML-B were compared. Cube plots indicated that ML lactose monohydrate, and DSCG combination gave the best performance (highest FPF) at high shear (SET-A); while SV lactose monohydrate and AS combination gave the worst performance at low shear (SET-D). Although an inverse relationship exists between ED and FPF in most cases, FPF is considered to be more important for evaluating formulation performance.

The IPB vs. FP formulations were also compared. The half-normal plots of ED values indicated that the largest contributing factor, $\operatorname{SET} \tau_{s}$, was significant for all ED comparisons. This suggested the large influence of $\tau_{s}$ on the flow properties. However, the largest contributing factor to FPF values was the drug factor (IPB and FP), which was significant in all formulations. When SV were compared with ML, there was significant difference between SV-A and ML-B, and between SV-B and ML-B. There were drug and $\tau_{s}$, drug/carrier interactions. No carrier effect was significant when batch-to-batch comparison was carried out between two SV or between two ML formulations. Cube plots of FPF indicated that ML-B and IPB combination gave the best performance (highest FPF) at highest $\tau_{s}$, while SV-A and FP combination gave the worst performance at lowest $\tau_{s}$. 


\subsubsection{Assessment of Aerosol Performance Using PADE}

It can be seen that the categorical comparison of aerosolization performance of different formulations using conventional method or DesignExpert analysis are quite inefficient and tedious. Here, a novel interpretation of drug aerosolization performance efficiency, the PADE, was developed by correlating performance with airflow parameter employed using SETs.[21] The utilize of this mathematical expression is based on: 1) The hypothesis that the model of molecular surface association described by surface adsorption expressions can be adapted to fit shear displacement observations; 2) The notion that drug particle removal from the surface of lactose carriers by shear force is analogous to surface site occupancy described in Langmuir adsorption isotherm, while the mechanism is analogous to molecular desorption. (see Chapter $\mathbf{5}$ for details of the analogy and difference between relevant physical phenomena such as surface adsorption, protein binding with similar algebraic equivalent expression). In this chapter, the mathematical expression defined below will be referred to as PADE:

$$
\begin{gathered}
\frac{F P F}{F P F_{\text {max }}}=\frac{k_{d} \tau_{s}}{1+k_{d} \tau_{s}} \\
\frac{\tau_{s}}{F P F}=\frac{\tau_{s}}{F P F_{\text {max }}}+\frac{1}{k_{d}\left(F P F_{\text {max }}\right)}
\end{gathered}
$$

where $F P F_{\max }$ is defined as the characteristic $F P F$ at shear stress $\tau_{s}$ approaches maxima and further increasing of $F P F$ would require comminution of the drug/carrier particles; $k_{d}$ is defined as the deaggregation constant, analogous to the Langmuir adsorption constant. 
The PADE parameters $\left(F P F_{\max }\right.$ and $\left.k_{d}\right)$, and regression goodness of fit $\left(R^{2}\right.$ and adjusted $R^{2}$ ) were determined.

The correlation of $F P F_{T D}$ or $F P F_{E D}$ with SET $\tau_{s}$ by PADE non-linear regression (Eq. 3-1) and PADE linear regression (Eq. 3-2) analyses are shown in Figure 3-7 and 3-8 for AS and DSCG formulations, and in Figure 3-9 and 3-10 for IPB and FP formulations, respectively. For PADE non-linear regression analyses, the coefficients $F P F_{\max }$ and $k_{d}$ for each formulation combination, as well as the goodness of fit $\left(R^{2}\right.$ and adjusted $\left.R^{2}\right)$ are shown in Table 3-2. An excellent goodness of fit was observed for all sixteen formulations based on the $R^{2}$, adjusted $R^{2}$ (adjusted $R^{2}: 0.8991-0.9993$ for AS and DSCG formulations, and 0.9140-0.9990 for IPB and FP formulation, with the exception of FP_ML-A formulation, adjusted $\left.R^{2}=0.7269\right)$. Higher $F P F$ represented greater drug deaggregation and performance efficiency. A correlation analogous to Langmuir surface adsorption was observed. Greater initial increase of $F P F$ was observed at low shear stress followed by smaller increases in $F P F$ at higher shear stress until $F P F$ approached to a plateau region at $F P F_{\max }$. Here, the $F P F_{\max }$ represented the maximum $F P F$ that could be achieved if the detachment shear stress reached maxima and in excess of the critical shear stress value. Further deaggregation of drug particles from carrier particles would not be possible because it would result in comminution of the drug/carrier particles. So $F P F_{\max }$ should be an important parameter for evaluating the performance efficiency of the aerosol formulations. The data interpreted by the foregoing mathematical approach showed that the rank order of performance efficiency based on $F P F_{\max }$ of the sixteen blends was: 
1) $\underline{\text { ML-B/DSCG }}>\underline{\text { ML-A/DSCG }}>\underline{\text { ML-A/AS }}>\underline{\text { ML-B/AS }}>\underline{\text { SV-B/DSCG }}>\underline{\text { SV-A/DSCG }}>$ $\underline{\mathrm{SV}-\mathrm{B} / \mathrm{AS}}>\underline{\mathrm{SV}-\mathrm{A} / \mathrm{AS}}$

2) $\underline{\text { ML-B/IPB }}>\underline{\text { ML-A/IPB }}>\underline{\text { SV-A/IPB }}>\underline{\text { SV-B/IPB }}>\underline{\text { ML-B/FP }}>\underline{\text { ML-A/FP }}>\underline{\text { SV-B/FP }}>$ $\underline{\text { SV-A/FP. }}$.

Both $F P F_{T D}$ and $F P F_{E D}$ give the same rank order. This rank order can be perceived more directly from the slopes $\left(1 / F P F_{\max }\right)$ of the PADE linear regression plots (Figure 3-9 and 3-10, Eq. 3-2). Excellent correlation was observed $\left(R^{2}: 0.9804-0.9998\right.$ for AS and DSCG formulations, and 0.9905-0.9999 for IPB and FP formulations, respectively). The steeper the slope, the poorer the performance efficiency is. In DSCG formulations, there exists a convergence point at $\tau_{s} \approx 1.9 \mathrm{~N} / \mathrm{m}^{2}$. In IPB formulations, the convergent point was at $\tau_{s} \approx$ 3.0 N/m² between SV-A, ML-A, and ML-B, and about $1.6 \mathrm{~N} / \mathrm{m}^{2}$ between SV-B and ML-B. In FP formulations, the convergent point also existed between SV-A, SV-B, and ML-A at $\tau_{s} \approx 1.8 \mathrm{~N} / \mathrm{m}^{2}$. The convergent points indicated that formulations with less performance efficiency had higher $F P F$ than more efficiently dispersed formulation below the critical $\tau_{s}$, whereas above this point, $F P F$ rank order reverses. In AS formulations, this convergence point is only observed in two SV blends at a higher shear $\left(\tau_{s} \approx 3.6 \mathrm{~N} / \mathrm{m}^{2}\right.$ ). It also implied that formulation heterogeneity may play a role, though the data fit and tolerate the PADE interpretation well. More data points are required to address this phenomenon. The interpretation of deaggregation constant $k_{d}$ is also related to the critical $\tau_{s}$. A more rigorous discussion of this novel and approach to particle dispersion data is presented in Chapter 5.[22] 


\subsubsection{ACI}

The fold over selection of blends for ACI studies was AS_SV-A, AS_SV-B, DSCG_ML-A, DSCG_ML-B; IPB_SV-A, IPB_SV-B, FP_ML-A, and FP_ML-B. The deposition pattern of these selected blends actuated at highest (SET-A) and lowest (SET-D) shear stress are shown in Figure 3-11. The aerosol dispersion efficiency was evaluated by ED, FPF and MMAD. (Table 3-3) The AS-SV formulation gave significantly lower FPF than DSCG-ML formulation, as was exemplified by the trend in deposition shifting to the lower stages (smaller particle sizes). SV formulations gave smaller MMAD than ML formulations. When the worst, SV-AS, formulation was actuated at lowest shear stress, the $F P F_{4.5}$ was 9.4-12.0\%. While the best, ML-DSCG, formulation was actuated at highest shear stress, a significant increase of $F P F_{4.5}$ was obtained (33.9-35.2\%). This can be considered as "matched" formulation and shear stress. However, a "mismatched" formulation and shear stress resulted in a lower but comparable $F P F_{4.5} . \quad(15.7-21.6 \%$ for $\mathrm{AS} / \mathrm{SV} / \mathrm{high}$ shear and $13.9-15.1 \%$ for DSCG/ML/low shear).

For IPB and FP formulations, the ED was influenced greatly by $\tau_{s}$. SET-D gave significantly higher ED values (87.0-91.6\%) than SET-A (56.0-77.2\%). Carrier effect was confounded by $\tau_{s}$ effect. IPB-SV formulation gave significantly higher FPF values than FP-ML formulation in both high shear $\left(\mathrm{FPF}_{4.5}\right.$ for IPB-SV: 20.9-23.2\%; $\mathrm{FPF}_{4.5}$ for FP-ML: 11.9-14.9\%) and low shear $\left(\mathrm{FPF}_{4.5}\right.$ for IPB-SV: 15.3-17.3\%; FPF 4.5 for FP-ML: 6.0-8.2\%), as exemplified by the deposition shifting to the lower stages. Higher $\tau_{s}$ and ML formulation resulted in the deposition to the lower stages than lower shear and SV 
formulation, but the main influence of FPF was the drug effect. The highest $\tau_{s}$ gave smaller MMAD values than those at the lowest $\tau_{s}(p<0.05)$. Both IPB-SV and FP-ML formulation gave similar MMAD values at the highest $\tau_{s}$, but FP-ML formulation gave significantly smaller MMAD values than IPB-SV formulation at low $\tau_{s} \cdot(p<0.05)$. Similar statistical analyses were performed and the findings are consistent with the TSLI data analysis.

Similarly, PADE applied to inhalation aerosol dispersion can be assessed. PADE linear regression can conventionally be simplified. (similar to the specific surface area determination of a solid from $\mathrm{N}_{2}$ adsorption isotherms, which could conventionally be analyzed by a single point method.[23]) For the present interpretation, a dual-point analysis (high shear and low shear) was conducted (Figure 3-12). The rank order from the slope of the lines indicated:

$$
\begin{aligned}
& \text { ML-B/DSCG } \geq \text { ML-A/DSCG }>>\text { SV-B/AS }>\text { SV-A/AS, } \\
& \text { and } \quad \text { SV-A/IPB }>\text { SV-B/IPB }>\text { ML-B/FP }>\text { ML-A/FP. }
\end{aligned}
$$

which is in good agreement with the above TSLI analysis. This simple method can be used to quickly estimate the formulation performance within the desired shear stress range.

\subsection{Discussion}

\subsubsection{Influence of Drugs}




\subsubsection{AS vs. DSCG}

The physicochemical nature of drugs is important to the formulation performance efficiency. AS particle surfaces are relatively smooth and non-spherical. DSCG particles are more irregularly shaped and surface defects (potentially "high surface energy" sites) was observed at high magnifications in SEM. The interparticulate adhesion forces were previously reported to be stronger for pure carrier-free DSCG than AS, which could be attributed to spontaneous self-assembly behavior of pure DSCG and liquid crystalline formation.[24] DSCG is an amphoteric chemical posssessing both hydrophobic and hydrophilic moieties. This surface-active drug is known to possess liquid-crystalline self-assembling properties and demonstrate liquid crystalline phase behavior.[25, 26] Bulk characterizations using DSC demonstrated characteristic liquid crystalline thermogram which indicated molecular long-range vs. short range order for micronized DSCG in the solid-state. Bulk characterization using XRPD confirmed the existence of partially ordered and disordered liquid crystalline mesophase in the micronized DSCG. DSC thermograms also indicated that the micronization of DSCG by jet-milling introduced molecular disorder. However, DSCG/lactose monohydrate blends gave superior aerosol performance leading to greater drug aerosolization performance than AS/lactose monohydrate blends.[27] The superior aerosol performance may be attributed to the strong interfacial energy-reducing properties of DSCG at polar lactose monohydrate interface. It is generally recognized that liquid crystalline materials inherently possess surface activity and surface tension reducing properties of high-energy surface such as water $(\gamma=72 \mathrm{mN} / \mathrm{m})$ and form stable soluble monolayers at air-water 
interface. $[8,9,25]$ These interfacial thermodynamic fact of DSCG appeared to reduce the high interfacial interactions occurring at the drug-carrier interface and resulted in better aerosol performance compared with AS. Besides, the electrostatic compatabiltiy between AS (positively charged) and lactose monohydrate (negatively charged) may also be a factor for poorer performance of AS formulation.[2] Furthermore, AS formulations were more susceptible to carrier change (SV vs. ML) than DSCG formulations, indicating stronger influence of ternary inclusion (intrinsic fines of ML) to the performance of AS formulations. It also suggested that AS formulations be more suitable for aerosolization performance mechanism studies of ternary-inclusion formulation. It is well-known that the inclusion of fines (typically less than $10 \mu \mathrm{m}$ ) improves formulation performance, though the performance mechanism following ternary blending is still debatable.[28] A balance of drug detachement from the carrier and deagglomeration of drug-drug or drug-fine lactose has been suggested recently.[29] It suggested that the micronized AS adhered to a greater extent to the major carrier particle surfaces than the micronized DSCG, which somehow detached from the major carrier surfaces, as was suggested by the increased fine particles after blending. It was further suggested that the balance of drug detachment from the carrier and deagglomeration could be different between AS and DSCG formulations. The balance could also be affected to a different extent if AS or DSCG concentration was changed. Because the available surface sites on the lactose monohydrate and the work of cohesion/adhesion for the drug and lactose mixture could result in different mixture structures.

\subsubsection{IPB vs. FP}


The physicochemical nature of drugs may strongly influence the formulation performance efficiency. The drug effect was the primary effect influencing the $F P F$ values for IPB and FP formulations. After milling, the morphology of FP was greatly changed from smooth and elongated particles to rough and irregular-shaped particles, as was indicated by SEM. Aggregation was observed in both milled IPB and FP. The particle size distribution was narrow and median particle diameter of IPB and FP were similar, by LD. The thermoanalysis using DSC indicated a substantial influence of jet-milling and blending on the powder formulation. A partial loss of bound water from rigid crystalline IPB was observed, which indicated the generation of some anhydrous ipratropium bromide, and creation of crystalline defects and increased molecular disorder during milling. The change of FP by milling was not reflected in DSC thermograms. The DSC thermograms showed peak broadening in all IPB, FP/lactose monohydrate blends, indicating the influence of blending to the powder formulation. The surface roughness of both IPB and FP blends introduced by milling and blending may also contribute to the differences in drug deaggregation. The superfine particles adhered to the primary FP following milling may also be an important factor. Jet-milling may not only induce surface defects but also possibly introduce electrostatic charge. Through molecular modeling of surface charge density on IPB, it was reported that IPB has a predominantly neutral surface (with some small regions of positive and negative charge).[30] Consequently, weak long range electrostatic interaction was observed between IPB and negatively charged lactose monohydrate.[30] The charge of micronized dry powder FP has not been fully elucidated, but it has been reported that the cohesive-adhesive balance ratio between FP-FP and 
FP-lactose monohydrate using micronized FP was quite small by AFM colloidal approach, indicating significant adhesion between micronized FP particle and lactose monohydrate.[31] This may explain the poor aerosolization performance exhibited by FP formulations. FP is a fluorinated corticosteroid that has a conjugated propionate moiety (Figure 1B). It is quite different from the ionic salt monohydrate IPB (Figure 1A) which has rigid crystalline monohydrate structure. The hydrophobic effect of FP due to the corticosteroid rings, the fluorine atoms, and the propionate moiety may strongly influence the interfacial properties and the subsequent aerosolization. The increased adhesion force may be explained by the increased contact area between FP and lactose monohydrate particles. Higher density of FP particle may also reduce the deaggregation due to inertia.

\subsubsection{Influence of Carriers}

\subsubsection{Carriers that blended with $A S$ vs. with DSCG}

Lactose monohydrate batches are also important factors that influenced formulation performance. The differences between lactose monohydrate batches includes particle size distribution, surface roughness, amorphous content, crystallinity, and intrinsic fine particles. The SEM images demonstrated that ML lactose monohydrates had much more intrinsic fines $(<10 \mu \mathrm{m})$ which appeared to agglomerate between the interstices of major carrier particles instead on the particle surfaces. The distribution of intrinsic fines remained in the interstices after blending with $2 \%(\mathrm{w} / \mathrm{w})$ drugs. The particles of SV lactose monohydrates were more regularly shaped and surfaces were smoother than those of ML lactose monohydrates, though their primary particle sizes were comparable.[13, 27] 
DSC thermograms and XRPD diffractograms revealed higher amorphous content and lower crystallinity of the ML lactose monohydrate batches compared to SV samples. These properties influenced the interparticulate forces, resulting in improved flow properties of $\mathrm{SV}$ formulations than $\mathrm{ML}$ formulations. Thus, superior $E D$ of $\mathrm{SV}$ formulations observed in performance studies across all SET airflow parameters. The intrinsic lactose monohydrate surface fines could serve as ternary components, responsible for the increase in $F P F$.[32] The statistical experimental data analyses indicated that SV-A and SV-B lactose monohydrate batches did not result in significant performance difference, nor did ML-B and ML-B lactose monohydrate batches. However, the performance efficiency between two SV or between two ML lactose monohydrates can be differentiated by using PADE. The TSLI and ACI were in good agreement to each other.

\subsubsection{Carriers that blended with IPB vs. with FP}

The lactose monohydrate batches had a great influence on powder fluidization and entrainment. This may be illustrated by the higher $E D$ values observed for SV blends, and lower ED values for ML blends. Carrier demonstrated a smaller effect in drug deaggregation for IPB formulations than FP formulations. For example, although SV-A and ML-A are known to be different due to greater content of intrinsic fines, surface roughness, and amorphous content of the latter, the $F P F$ values were similar at high shear stress for IPB formulations. However, the FP formulations exhibited much greater carrier effect than IPB formulations. The FPF values were significantly larger for ML blends than SV blends. This may be explained by the agglomeration mechanism for ternary 
component. Micronized FP was known to strongly adhere to the lactose monohydrate, which was advantageous in forming larger agglomerates unlike the less adhesive micronized IPB, resulting in a large difference in performance between SV and ML formulations. Larger agglomerates were believed to be subject to greater deaggregation force.[28, 31]

\subsubsection{Influence of Shear Stress}

Different devices may have distinct mechanism of deaggregation which cause aerosol particle separation by 1) aerodynamic 'pull-off' or tangential displacement;[33] 2) mechanical impaction;[34] 3) twisting 'torque' force that have been emphasized.[35] Conversely, the entrainment occurs in SETs by turbulence, collision, and to a large extent, shear force were not paid enough attention. The turbulent shear stress is believed to be the principal mechanism of deaggregation within the SETs overcoming interparticulate forces leading to formulation fluidization and deaggregation.[34, 36] Higher shear leads to higher degree of drug deaggregation and better aerosolization performance efficiency. The magnitude of shear stress is dependent on the specific resistance and patient's inspiratory flow.[37] The specific resistance and the flow rate should be within a patient's normal range of use. The specific resistance encompasses the values based on commercial inhalers.[14] The intent of the aerosolization performance studies was to challenge the formulation independently of the device, as a logical first step in product development. In order to do this it is desirable to employ as simple a method of particle stripping as 
possible to challenge the association between drug and carrier. Undoubtedly, in the final product the impaction and momentum transfer are important, perhaps the most important, mechanisms for which a device is designed. However, it is reasonable to suggest that particles which can be stripped by shear forces may more readily be removed under the influence of impaction and momentum transfer, from which it can be inferred that these formulation performance studies may extrapolate qualitatively (as a rank order for example) to drug product (device and formulation) performance.

The $E D$ represents the degree of powder entrainment, where higher $E D$ values indicate greater entrainment of the drug dose.[14] All SETs have an expansion region to allow for reduction in energy and to limit impaction in the inlet of the sampling device. The lower shear SET-C and D were designed to have gradual enlargment to facilitate gradual energy loss, and centrally located dosing platform to maximize airflow velocity, whereas SET-A and B were designed to have high shear which did not require consideration of reduction in energy loss as SET-C and D.[14] Other design features such as requirement for a much longer connection to the inlet of the sampling device or a larger angle for a diffuser for energy recovery contributed to the decision to not include a cone diffuser on SET-A and B. This resulted in higher ED in low shear SET-C and D and lower ED in higher shear SET-A and B, which is consistent with previous report.[14] This indicates that there are two portions of the entrained dry powders: a major portion that involves in particle deaggregation and a minor portion that remains in the SETs without undergoing deaggregation. The minor portion in SET-A and B was more pronounced than that in SET-C and D, due to the SET design and resulted in reduced ED. 
It is important to note that $E D$ described in this context is a portion of the emitted, or delivered, dose usually seen from inhalers. The portion that is deposited in the SET expansion outlet would deposit in the inlet of the sampling device (TSLI, ACI) if delivered from an inhaler. Such phenomena may explain that aerosol dry powder formulations having similar in vitro FPF values could exhibit quite different in vivo lung deposition.[38] The ability to discriminate between the portion of the delivered dose that is or is not deaggregated is a useful feature of the SETs since it points exclusively to formulation performance in the absence of specific inhaler design characteristics.

The degree of deaggregation was represented by either $F P F_{T D}, F P F_{E D}$, where larger FPF represented greater deaggregation. The correlation of FPF and SET airflow parameters indicated that increased $F P F$ were obtained using higher shear SETs at given airflow, and hyperbola-shaped relationships were observed in all formulation blends. Greater initial increases in FPF were observed at low shear stress, and small increases in $F P F$ at higher shear stress, until reaching a plateau. This suggests that aerosolization at higher shear stress will result in reduced actuation variability. According to the deaggregation mechanism, the shear stress applied overcomes the interparticulate forces that occur at the micronized pulmonary drug-sugar carrier interface i.e. solid-solid interface. It has been demonstrated systematically that the expression for surface interactions describing physical phenomena at an interface at the molecular level can also be applied to the aerosol particulate systems. The PADE appears to be a suitable and physically meaningful method facilitating interpretation of the aerosol performance data. This method, when adapted for pharmaceutical aerosolization performance, can be 
directly related to the physical interactions occurring at the interface. Particles being removed with increasing difficulty because of the sites that they occupy is analogous to surface site occupancy described in Langmuir adsorption isotherm. The Langmuir adsorption isotherm is the cornerstone of almost all theories of surface interactions on a molecular scale. Many theoretical or semi-empirical surface adsorption models that take into account the surface heterogeneity and molecular lateral interaction could possibly be modified to describe aerosol performance.[39] More rigorous studies that consider particle heterogeneity and/or lateral interaction will be discussed in Chapter 5.[22] Furthermore, PADE linear regression can be further simplified to dual point analysis for quick comparison of aerosolization performance efficiency, which in the present studies also proved to be valid.

\subsection{Summary}

This chapter focuses on the physicochemical characterization and performance studies of well characterized aerosol formulations using a range of techniques, and using SETs that encompass a range of airflow parameters such as shear stress consistent with commercial device performance, to characterize and evaluate the aerosol formulation performance in a device independent manner. A fold-over statistical experimental design was employed that could later be used to compare and search for any other interactions that may exist. The formulation preparation involved micronization of drug particles, and interactive physical blending of micronized pulmonary drugs with coarser lactose monohydrate 
carriers. SETs with well defined airflow parameters were employed and proved to be useful and device-independent screening tools for formulation performance study. Three important factors including: drug, carrier lactose monohydrate, and shear stress (SET) were compared and the performance efficiency was evaluated by statistical experimental design. The TSLI and ACI data demonstrated that the best performing blend in AS and DSCG systems (highest FPF, smallest and narrowest particle size distribution) was DSCG in ML lactose monohydrate batches at high shear stress, whereas the worst performing blend was AS in SV sample at low shear. The best performing blend in IPB and FP systems was IPB in ML lactose monohydrate batches at high shear, whereas the worst performing blend was FP in SV at low shear. There appeared to be an inverse relationship between $E D$ and $F P F$, but $F P F$ is a more important measure of the aerosolization performance efficiency since it is closely related to the actual pulmonary delivery of drugs. SET has the capability of differentiating the portion of blend that deaggregates from the portion that does not deaggregate when the blend is entrained in the SETs. A correlation of the SET shear stress with aerosol performance led to a new interpretation of particulate deaggregation, PADE, similar to molecular association such as surface adsorption exemplified here by Langmuir adsorption. Other interpretations such as protein binding equivalent or other more rigorous surface adsorption models that consider surface heterogeneity or lateral interaction may possibly be applied to the aerosol formulation depending on the specific systems and the performance evaluation results. Here, the PADE and its linear regression have been used as a meaningful method to rigorously and quickly select the formulation for prospects of good performance 
efficiency while alleviating the necessity, in the first instance, to delve onto various individual forces acting on each individual particle or enter into device considerations. The PADE method using two-parameter Langmuir equivalent expression results in excellent goodness of fit and which facilitiates a physical interpretation, and may be used to evaluate dry powder formulations given similar deaggregation mechanisms applied by SETs. The dual-point analysis can greatly expedite the performance screening and prediction of aerosol formulations. 
Table 3-1. Particle volume size distribution of albuterol sulfate (AS), disodium cromoglycate (DSCG), ipratropium bromide monohydrate (IPB), and fluticasone propionate (FP) before and after air jet-milling. (Mean $\pm \mathrm{SD}$ ).

\begin{tabular}{|l|c|c|c|c|c|}
\hline & Span & $\mathbf{D}_{\mathbf{1 0}}(\boldsymbol{\mu m})$ & $\mathbf{D}_{\mathbf{5 0}}(\boldsymbol{\mu m})$ & $\mathbf{D}_{\mathbf{9 0}}(\boldsymbol{\mu m})$ & $\mathbf{D}_{\mathbf{a e}}(\boldsymbol{\mu m})$ \\
\hline Micronized AS & $0.26 \pm 0.09$ & $3.20 \pm 0.06$ & $3.59 \pm 0.13$ & $4.13 \pm 0.40$ & - \\
\hline Micronized DSCG & $0.32 \pm 0.01$ & $3.24 \pm 0.01$ & $3.68 \pm 0.02$ & $4.40 \pm 0.04$ & - \\
\hline IPB unmilled* & $2.29 \pm 0.02$ & $3.64 \pm 0.10$ & $9.26 \pm 0.19$ & $24.87 \pm 0.69$ & 10.68 \\
\hline IPB once-milled* & $0.23 \pm 0.04$ & $4.12 \pm 0.05$ & $4.53 \pm 0.16$ & $5.16 \pm 0.28$ & 5.22 \\
\hline IPB twice-milled* & $0.34 \pm 0.05$ & $2.82 \pm 0.21$ & $3.29 \pm 0.24$ & $3.94 \pm 0.20$ & 3.79 \\
\hline FP unmilled* & $1.92 \pm 0.09$ & $11.39 \pm 1.28$ & $38.31 \pm 5.40$ & $85.01 \pm 12.65$ & 44.18 \\
\hline FP twice milled* & $0.36 \pm 0.22$ & $3.87 \pm 0.20$ & $4.47 \pm 0.09$ & $5.47 \pm 0.83$ & 5.16 \\
\hline
\end{tabular}


Table 3-2. TSLI performance data analysis. The relationship between SET shear stress $\left(\tau_{s}\right)$ and $F P F_{T D<6.4} / F P F_{E D<6.4}$ for IPB/lactose monohydrate and $\mathrm{FP} /$ lactose monohydrate dry powder formulations using non-linear regression analysis. $(n=3)$.

$$
\frac{F P F}{F P F_{\max }}=\frac{k_{d} \tau_{s}}{1+k_{d} \tau_{s}} \quad \text { (Eq. 3-1) }
$$

\begin{tabular}{|c|c|c|c|c|c|c|}
\hline & Drug & Lactose & FPF $_{\text {max }}$ & $\mathbf{k}_{\mathbf{d}}$ & $\mathbf{R}^{2}$ & $\operatorname{Adj} R^{2}$ \\
\hline \multirow{16}{*}{$\mathrm{FPF}_{\mathrm{TD}}$} & \multirow{4}{*}{ AS } & SV-A & 14.49 & 1.31 & 0.9739 & 0.9652 \\
\hline & & SV-B & 16.55 & 0.75 & 0.9362 & 0.9149 \\
\hline & & ML-A & 43.51 & 1.32 & 0.9893 & 0.9858 \\
\hline & & ML-B & 38.54 & 0.76 & 0.9691 & 0.9588 \\
\hline & \multirow{4}{*}{ DSCG } & SV-A & 35.59 & 3.00 & 0.9896 & 0.9861 \\
\hline & & SV-B & 37.40 & 3.75 & 0.9927 & 0.9902 \\
\hline & & ML-A & 44.78 & 1.32 & 0.9978 & 0.9970 \\
\hline & & ML-B & 48.62 & 1.30 & 0.9987 & 0.9983 \\
\hline & \multirow{4}{*}{ IPB } & SV-A & 32.99 & 25.22 & 0.9954 & 0.9939 \\
\hline & & SV-B & 29.51 & 80.02 & 0.9438 & 0.9250 \\
\hline & & ML-A & 34.02 & 5.26 & 0.9967 & 0.9955 \\
\hline & & ML-B & 37.56 & 2.11 & 0.9806 & 0.9741 \\
\hline & \multirow{4}{*}{ FP } & SV-A & 14.95 & 14.84 & 0.9823 & 0.9764 \\
\hline & & SV-B & 16.04 & 3.37 & 0.9993 & 0.9990 \\
\hline & & ML-A & 18.57 & 5.19 & 0.9000 & 0.8667 \\
\hline & & ML-B & 24.20 & 14.33 & 0.9954 & 0.9939 \\
\hline \multirow{16}{*}{$\mathrm{FPF}_{\mathrm{ED}}$} & \multirow{4}{*}{ AS } & SV-A & 18.03 & 0.93 & 0.9634 & 0.9513 \\
\hline & & SV-B & 21.45 & 0.45 & 0.9244 & 0.8991 \\
\hline & & ML-A & 54.05 & 1.21 & 0.9833 & 0.9778 \\
\hline & & ML-B & 50.44 & 0.68 & 0.9342 & 0.9123 \\
\hline & \multirow{4}{*}{ DSCG } & SV-A & 46.34 & 1.76 & 0.9995 & 0.9993 \\
\hline & & SV-B & 47.51 & 2.23 & 0.9995 & 0.9993 \\
\hline & & ML-A & 61.15 & 1.00 & 0.9904 & 0.9872 \\
\hline & & ML-B & 64.13 & 1.10 & 0.9851 & 0.9801 \\
\hline & \multirow{4}{*}{ IPB } & SV-A & 45.63 & 4.25 & 0.9910 & 0.9879 \\
\hline & & SV-B & 42.06 & 4.91 & 0.9804 & 0.9738 \\
\hline & & ML-A & 50.04 & 2.27 & 0.9731 & 0.9641 \\
\hline & & ML-B & 57.46 & 1.13 & 0.9456 & 0.9274 \\
\hline & \multirow{4}{*}{ FP } & SV-A & 21.27 & 3.58 & 0.9946 & 0.9929 \\
\hline & & SV-B & 23.16 & 1.53 & 0.9939 & 0.9919 \\
\hline & & ML-A & 34.27 & 1.22 & 0.7951 & 0.7269 \\
\hline & & ML-B & 37.91 & 2.88 & 0.9355 & 0.9140 \\
\hline
\end{tabular}


Table 3-3. ACI drug aerosolization performance (ED, FPF, and MMAD) studies via $\operatorname{SET}-\mathrm{D}\left(\tau_{\mathrm{s}}=0.624 \mathrm{~N} / \mathrm{m}^{2}\right)$ and SET-A $\left(\tau_{\mathrm{s}}=13.143 \mathrm{~N} / \mathrm{m}^{2}\right)$ at airflow rate $\mathrm{Q}=60 \mathrm{~L} / \mathrm{min}$. The formulations in comparison were SV-AS vs. ML-DSCG; and SV-IPB vs. ML-FP (mean values, $n=3$ with standard deviation in parenthesis).

\begin{tabular}{|c|c|c|c|c|c|c|c|}
\hline SET & Lactose-Drug & $\begin{array}{l}\text { ED } \\
(\%) \\
\end{array}$ & $\begin{array}{c}\mathrm{FPM}_{6.5} \\
(\mu \mathrm{m})\end{array}$ & $\begin{array}{c}\mathbf{F P M}_{4.5} \\
(\mu \mathrm{m})\end{array}$ & $\begin{array}{c}\mathrm{FPF}_{6.5} \\
(\%)\end{array}$ & $\begin{array}{c}\mathrm{FPF}_{4.5} \\
(\%)\end{array}$ & $\begin{array}{c}\text { MMAD } \\
(\mu \mathrm{m})\end{array}$ \\
\hline \multirow{4}{*}{$\begin{array}{c}\text { SET } \\
\text { A }\end{array}$} & SV-A_AS & $86.6(2.6)$ & $192.9(6.8)$ & $168.1(6.2)$ & $18.1(0.2)$ & $15.7(0.2)$ & $1.56(0.04)$ \\
\hline & SV-B_AS & $92.1(1.4)$ & $225.5(45.6)$ & $204.6(42.3)$ & $23.8(5.9)$ & $21.6(5.4)$ & $1.45(0.07)$ \\
\hline & ML-A_DSCG & $82.1(0.7)$ & $446.3(43.8)$ & $327.6(28.5)$ & $46.1(0.3)$ & $33.9(0.8)$ & $2.52(0.12)$ \\
\hline & ML-B_DSCG & $82.6(2.0)$ & $459.7(34.3)$ & $340.3(26.2)$ & $47.6(2.5)$ & $35.2(2.0)$ & $2.54(0.14)$ \\
\hline \multirow{4}{*}{$\begin{array}{c}\text { SET } \\
\text { D }\end{array}$} & SV-A_AS & $87.8(3.3)$ & $130.5(22.5)$ & $104.2(18.2)$ & $11.8(1.8)$ & $9.4(1.4)$ & $1.73(0.33)$ \\
\hline & SV-B_AS & $89.2(4.2)$ & $156.4(49.2)$ & $126.3(39.1)$ & $14.9(4.4)$ & $12.0(3.5)$ & $1.76(0.14)$ \\
\hline & ML-A_DSCG & $81.8(10.5)$ & $205.9(28.2)$ & $134.3(17.1)$ & $21.3(3.1)$ & $13.9(1.8)$ & $2.88(0.13)$ \\
\hline & ML-B_DSCG & $88.0(3.0)$ & $223.3(18.3)$ & $144.6(15.0)$ & $23.4(1.9)$ & $15.1(1.5)$ & $3.02(0.19)$ \\
\hline \multirow{4}{*}{$\begin{array}{c}\text { SET } \\
\text { A }\end{array}$} & SV-A_IPB & $77.2(1.9)$ & $357.5(12.7)$ & $247.1(11.2)$ & $33.5(2.4)$ & $23.2(1.5)$ & $2.57(0.34)$ \\
\hline & SV-B_IPB & $75.2(4.4)$ & $302.7(57.0)$ & $205.0(47.6)$ & $30.8(3.0)$ & $20.9(3.1)$ & $2.86(0.45)$ \\
\hline & ML-A_FP & $56.0(3.1)$ & $142.5(11.0)$ & $102.8(9.1)$ & $16.4(1.5)$ & $11.9(1.2)$ & $2.62(0.09)$ \\
\hline & ML-B_FP & $64.3(1.8)$ & $180.3(9.6)$ & $128.6(4.6)$ & $20.9(1.3)$ & $14.9(0.7)$ & $2.54(0.10)$ \\
\hline \multirow{4}{*}{$\begin{array}{c}\text { SET } \\
\text { D }\end{array}$} & SV-A_IPB & $91.6(1.5)$ & $276.2(18.6)$ & $166.0(18.0)$ & $25.3(4.0)$ & $15.3(3.2)$ & $3.26(0.39)$ \\
\hline & SV-B_IPB & $91.3(1.9)$ & $275.2(36.2)$ & $168.7(31.0)$ & $28.2(2.8)$ & $17.3(2.7)$ & $3.21(0.37)$ \\
\hline & ML-A_FP & $87.0(1.7)$ & $82.1(10.8)$ & $53.2(7.7)$ & $9.2(1.1)$ & $6.0(0.7)$ & $2.71(0.09)$ \\
\hline & ML-B_FP & $87.5(2.0)$ & $112.3(17.8)$ & $75.0(11.3)$ & $12.3(1.7)$ & $8.2(1.1)$ & $2.84(0.11)$ \\
\hline
\end{tabular}


Figure 3-1. Chemical Structures of (A) Albuterol sulfate; (B) Disodium cromoglycate; (C) Ipratropium bromide monohydrate; (D) Fluticasone propionate; (E) $\alpha$-lactose monohydrate.<smiles>CC(C)(C)NCC(O)c1ccc(O)c(CO)c1</smiles>

(C)

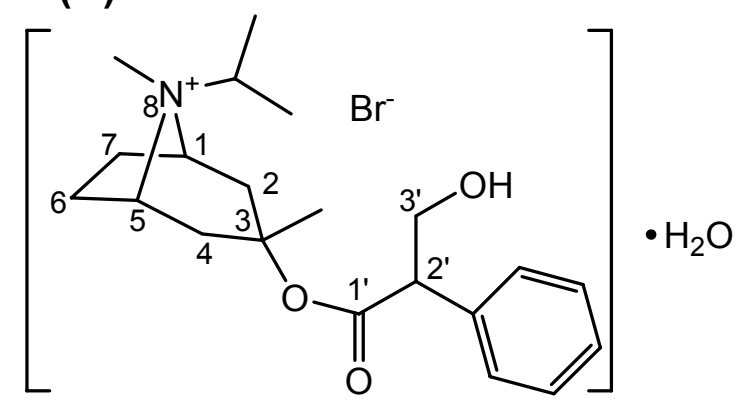<smiles>NC(=O)c1cc(=O)c2c(OCC(O)COc3cccc4oc(C(N)=O)cc(=O)c34)cccc2o1</smiles>

(B)

(D)<smiles>CCC(=O)O[C@]1(C(=O)SCF)[C@@H](C)C[C@H]2[C@@]3(C)C[C@H](F)C4=CC(=O)C=C[C@]4(C)[C@]3(F)[C@@H](O)C[C@]21C</smiles><smiles>OC[C@H]1O[C@@H](O[C@@H]2[C@@H](CO)O[C@H](O)[C@H](O)[C@H]2O)[C@H](O)[C@@H](O)[C@@H]1O</smiles> 
Figure 3-2. Representative scanning electron micrographs of disodium cromoglycate (DSCG), ipratropium bromide monohydrate (IPB), and fluticasone propionate (FP) before and after micronization by air jet milling: (A) raw DSCG (3k $\times$ magnification); (B) micronized DSCG $(20 \mathrm{k} \times)$; (C) raw IPB $(3 \mathrm{k} \times)$; (D) raw FP $(150 \times)$; (E) IPB after $1^{\text {st }}$ milling (3kx); (F) FP after $1^{\text {st }}$ milling $(3 \mathrm{k} \times)$; (G) IPB after $2^{\text {nd }}$ milling $(10 \mathrm{k} \times)$; and $(\mathrm{H}) \mathrm{FP}$ after $2^{\text {nd }}$ milling $(10 \mathrm{k} \times)$.

(A)

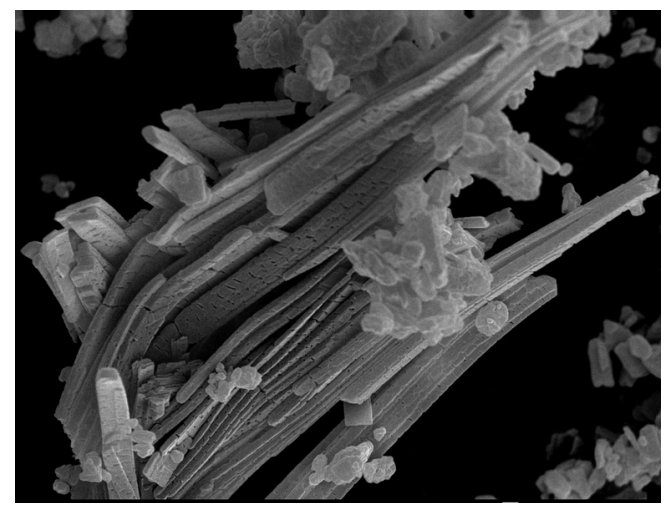

(C)

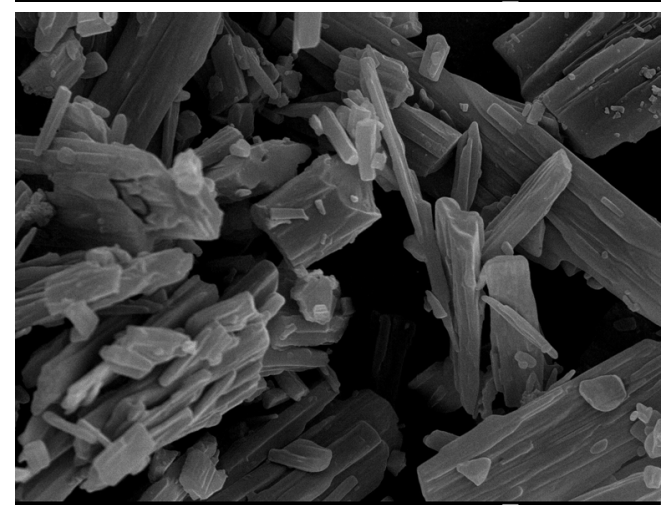

(E)

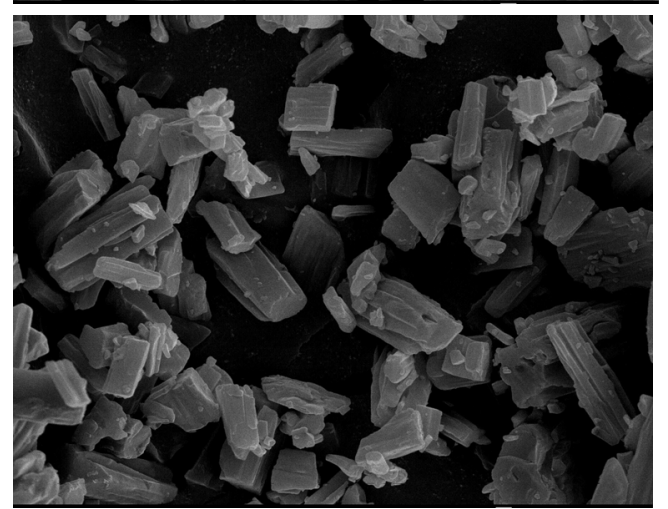

(G)

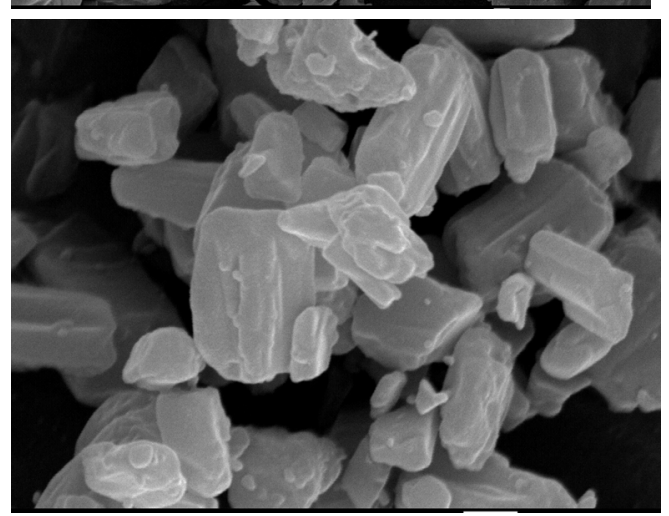

(B)

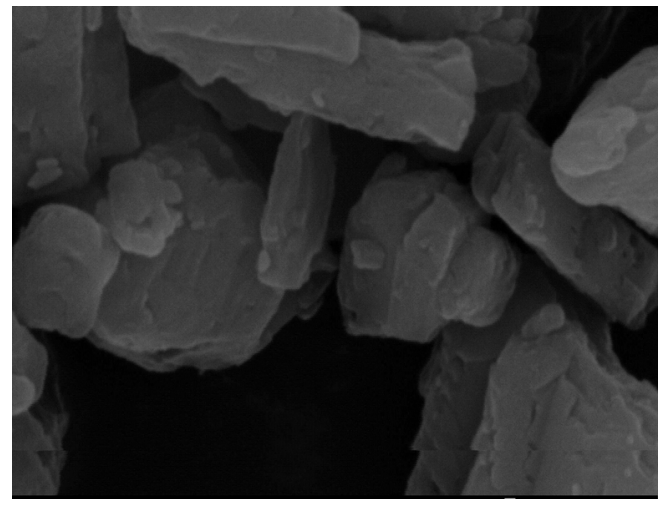

(D)

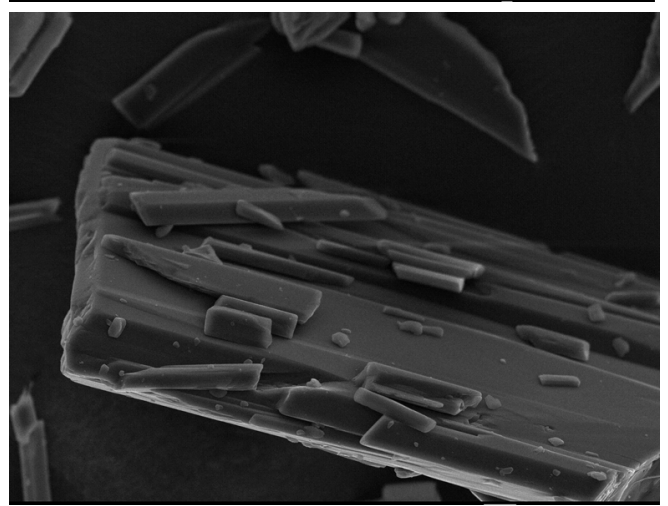

(F)

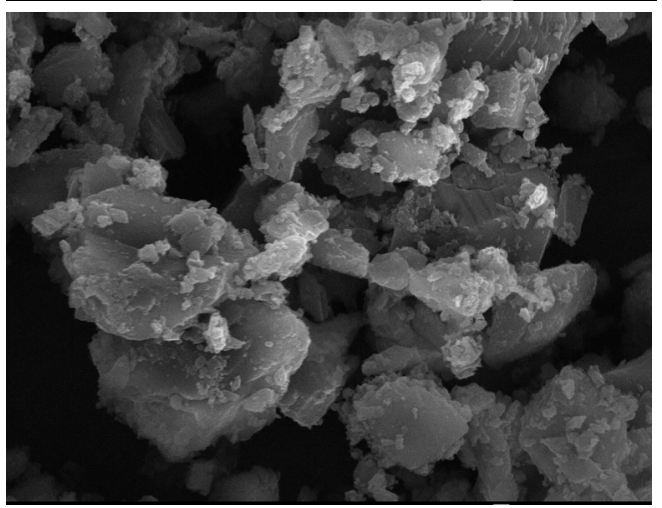

(H)

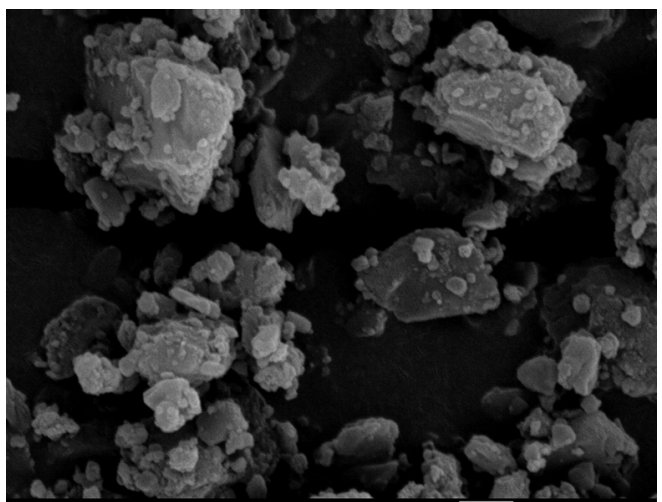


Figure 3-3. Representative SEM images of drug (DSCG, IPB, FP) and lactose monohydrate (2 sieved batches (SV) and 2 milled batches (ML)) interactive physical mixtures at 2\%w/w drug concentration: (A) DSCG-SV (1,500×); (B) DSCG-ML $(1,500 \times)$; (C) IPB-SV (1,500×); (D) IPB-ML (1,500×); (E) FP-SV (600×); and (F) FP-ML (600×). (See Chapter 2 for AS-lactose monohydrate blends.)

(A)

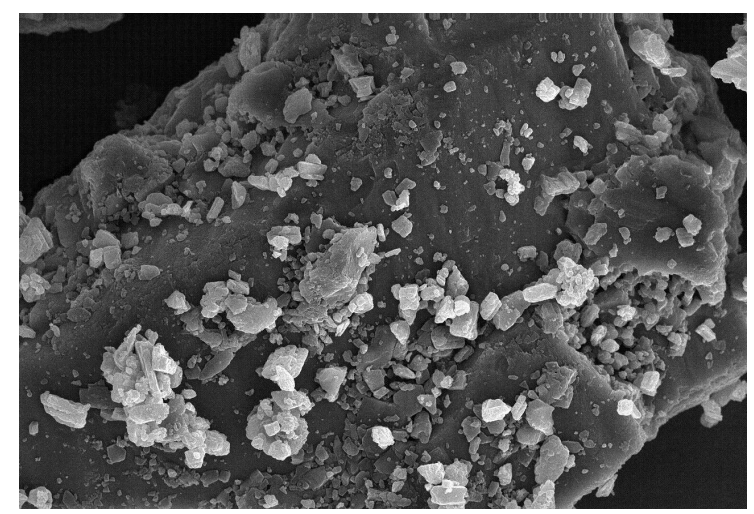

(C)

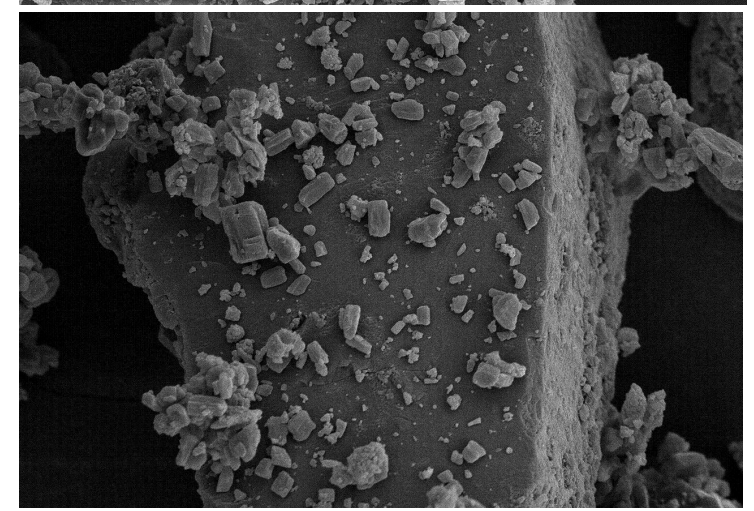

(E)

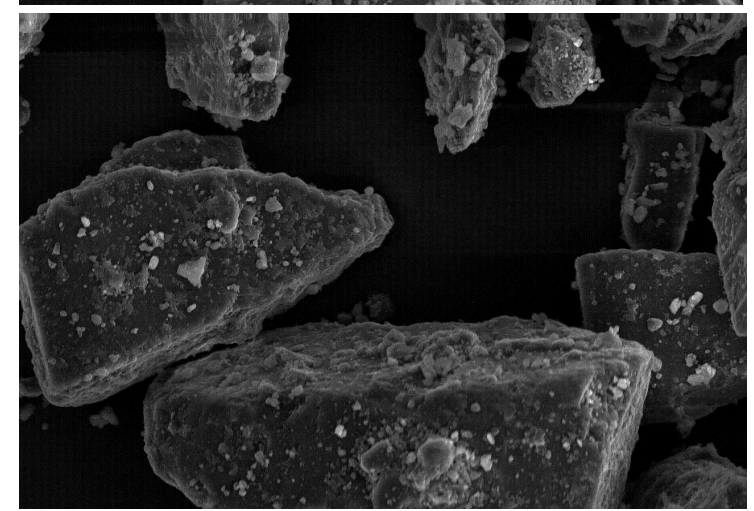

(B)

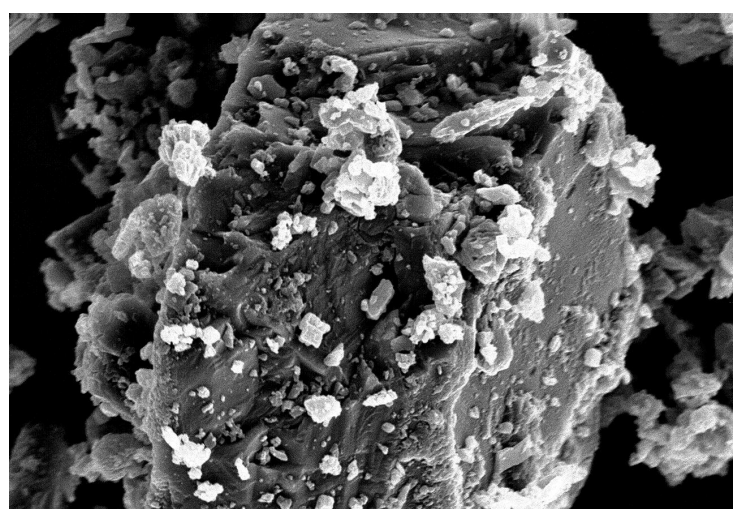

(D)

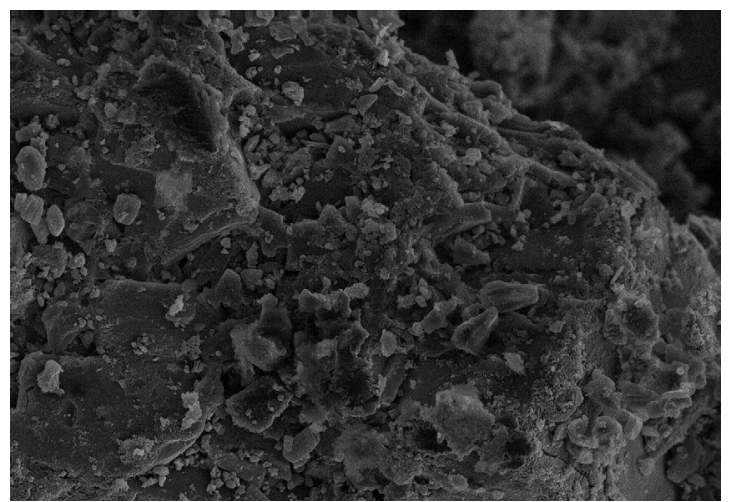

(F)

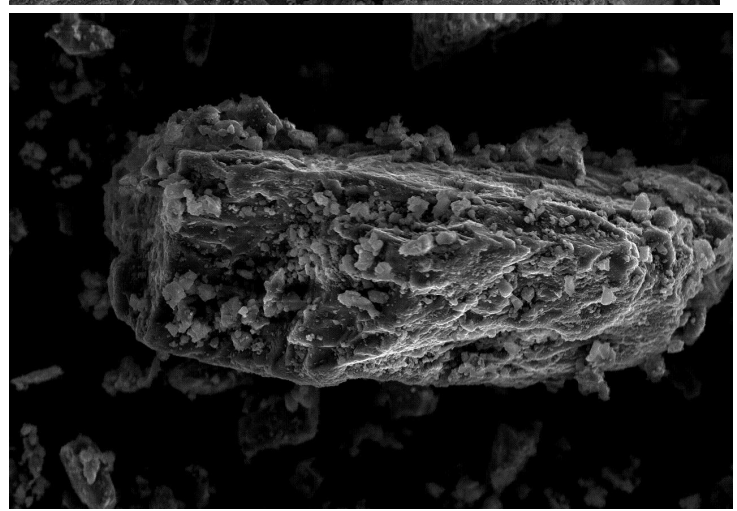


Figure 3-4. Representative Differential Scanning Calorimetry Thermograms of Raw Drugs and Drugs After Micronization by Jet-milling: (A) Unmilled and micronized DSCG; (B) Unmilled and micronized IPB; (C) Unmilled and micronized FP. Scanning rate is at $5^{\circ} \mathrm{C} / \mathrm{min}$.

(A)

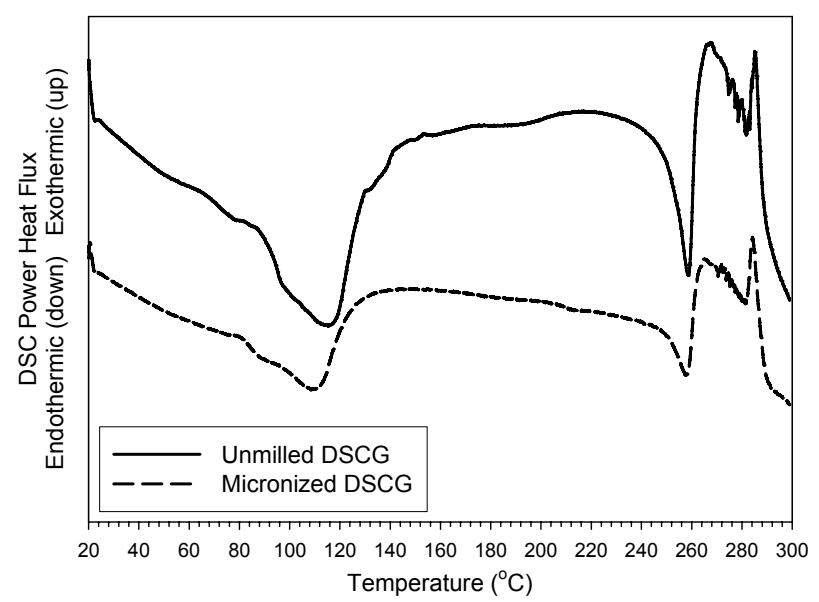

(B)

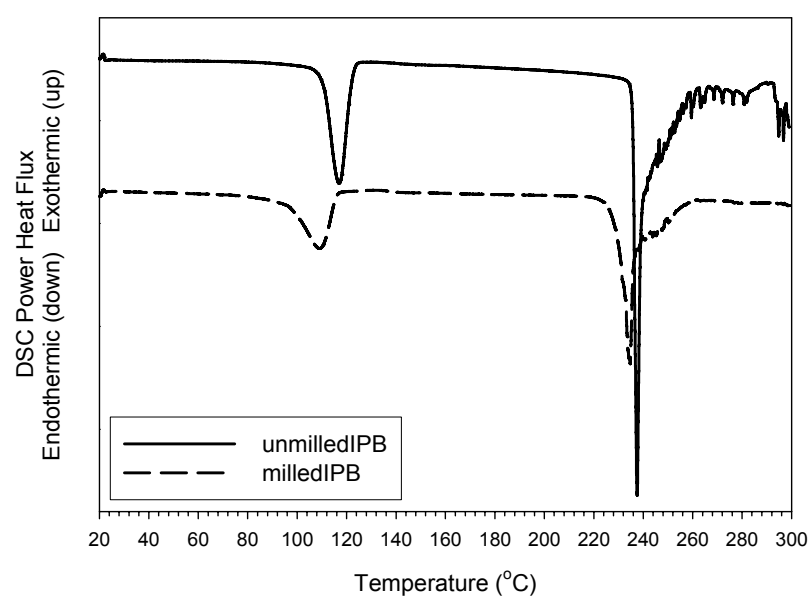

(C)

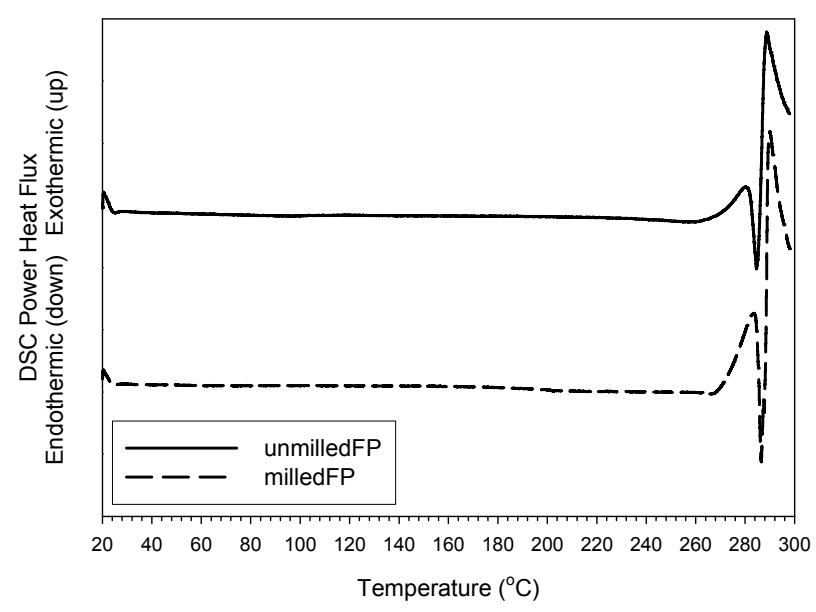


Figure 3-5. Representative DSC thermograms of drug-lactose monohydrate (SVs or MLs) interactive physical mixtures: (A) blends of DSCG $(2 \% \mathrm{w} / \mathrm{w})$ with SV-A and SV-B, respectively; (B) blends of DSCG $(2 \% \mathrm{w} / \mathrm{w})$ with ML-A and ML-B, respectively; (C) blends of IPB $(2 \% \mathrm{w} / \mathrm{w})$ with SV-A and SV-B, respectively; (D) blends of IPB $(2 \% \mathrm{w} / \mathrm{w})$ with ML-A and ML-B, respectively; (E) blends of FP $(2 \% \mathrm{w} / \mathrm{w})$ with SV-A and SV-B, respectively; and $(\mathrm{F})$ blends of $\mathrm{FP}(2 \% \mathrm{w} / \mathrm{w})$ with ML-A and ML-B, respectively. Scanning rate is at $5{ }^{\circ} \mathrm{C} / \mathrm{min}$.
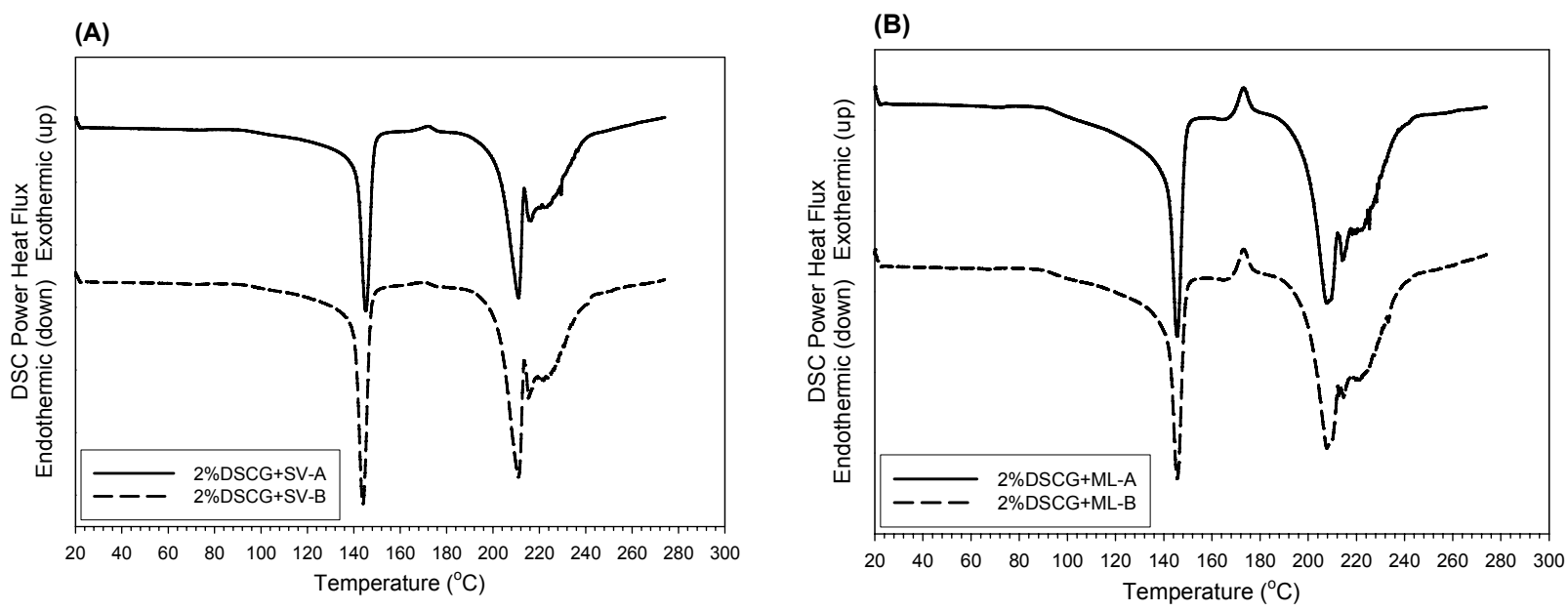

(C)

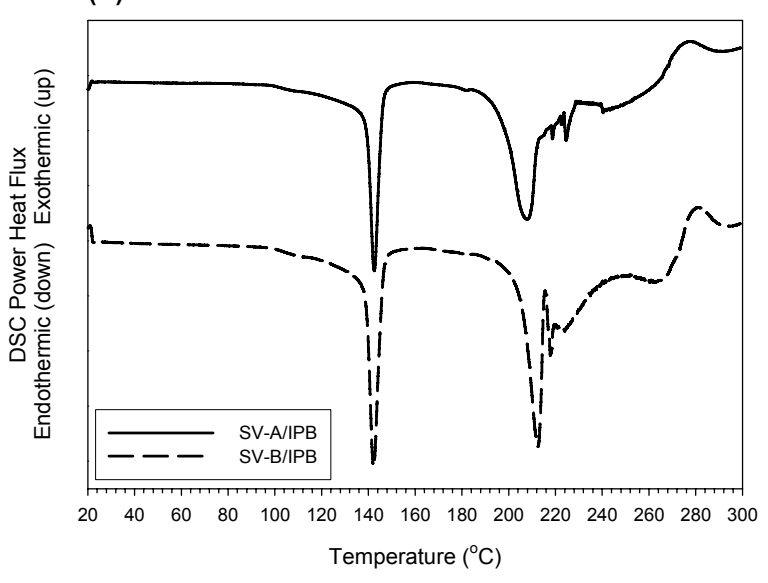

(D)

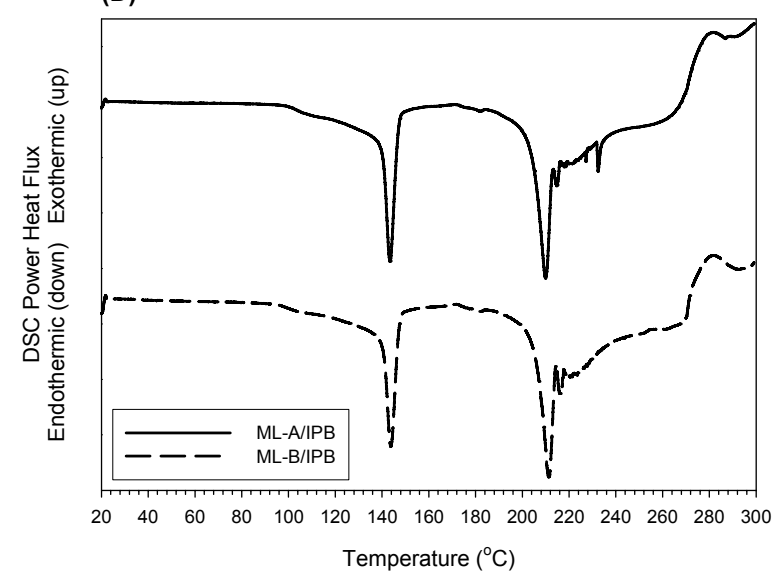

(E)

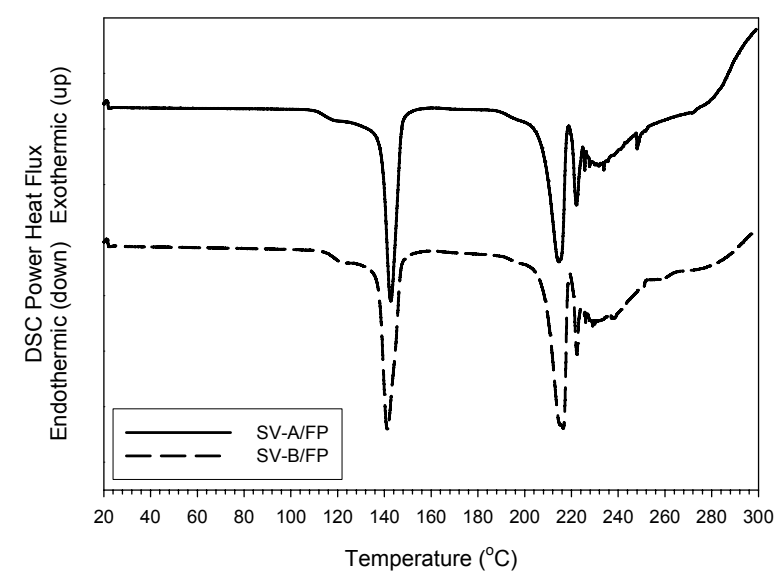


Figure 3-6. X-ray powder \%height intensity diffractograms of micronized drugs (A) albuterol sulfate; (B) disodium cromoglycate; (C) ipratropium bromide monohydrate; and (D) fluticasone propionate.

(A)

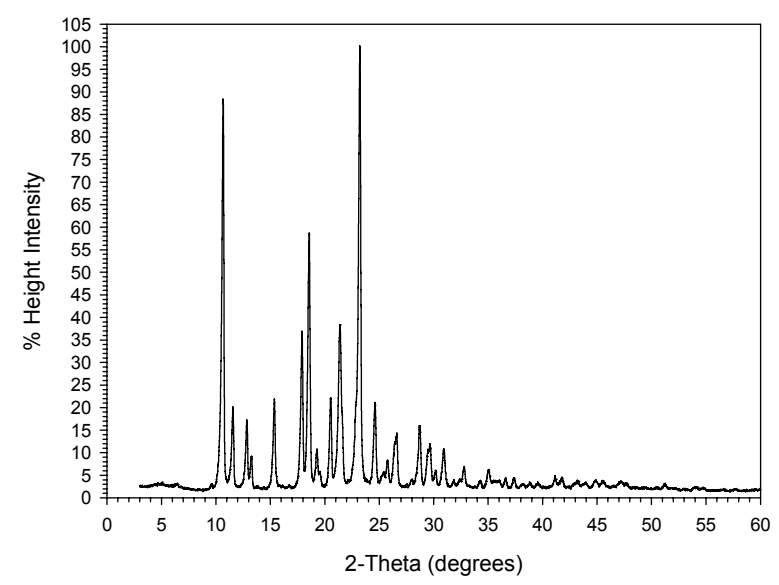

(C)

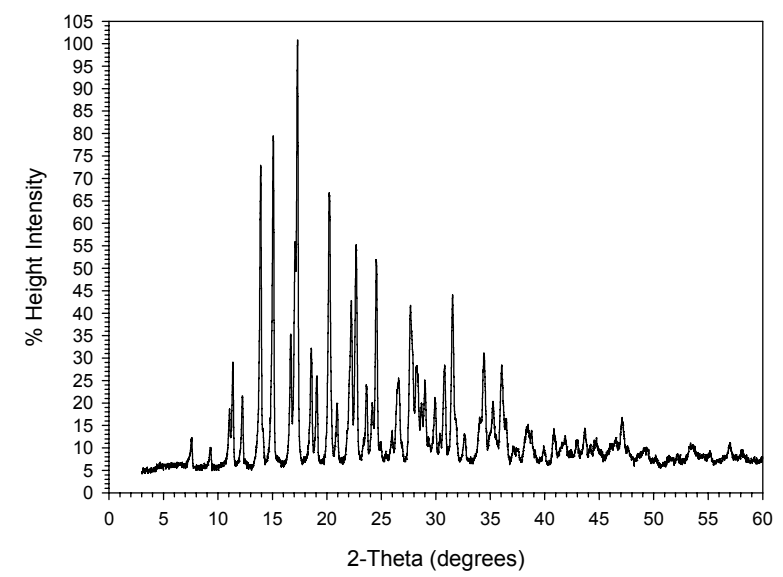

(B)

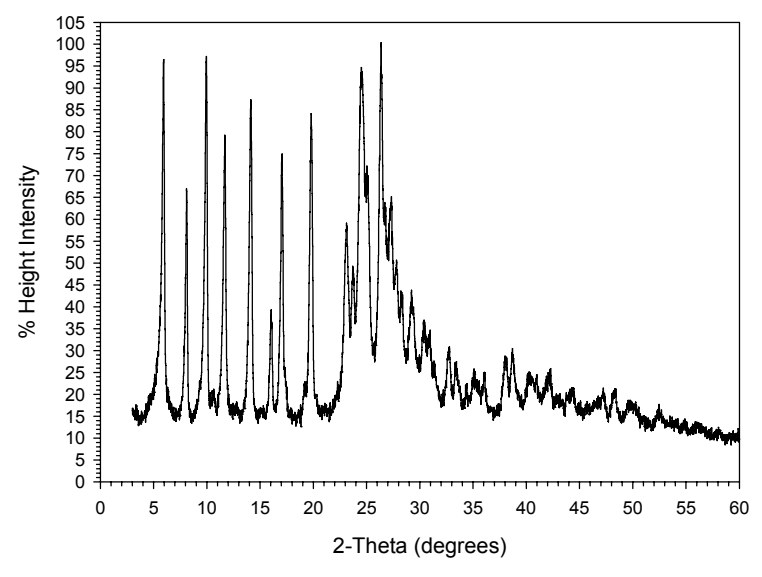

(D)

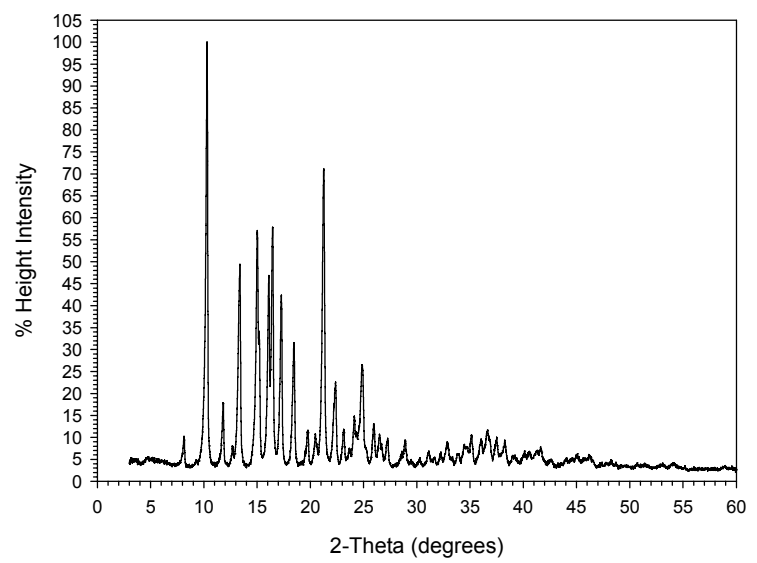


Figure 3-7. The relationship between SET airflow parameter (exemplified by shear stress $\left.\tau_{\mathrm{s}}\right)$ and TSLI formulation performance $\left(E D, F P F_{T D}, F P F_{E D}\right)$. Nonlinear regression analyses were applied using PADE for $F P F-\tau_{s}$ correlation (Eq.3-1). Left panels (A) to (C) are AS/lactose monohydrate systems (2\%w/w): (A) $E D-\tau_{s}$; (B) $F P F_{T D^{-}} \tau_{s}$ curve fitting; (C) $F P F_{E D^{-}} \tau_{s}$ curve fitting. Right panels (D) to (F) are DSCG/lactose monohydrate systems (2\%w/w): (D) $E D-\tau_{s}$; (E) $F P F_{T D^{-}} \tau_{s}$ curve fitting; (F) $F P F_{E D^{-}} \tau_{s}$ curve fitting. (Mean $\pm \mathrm{SD}$, $n=3)$.
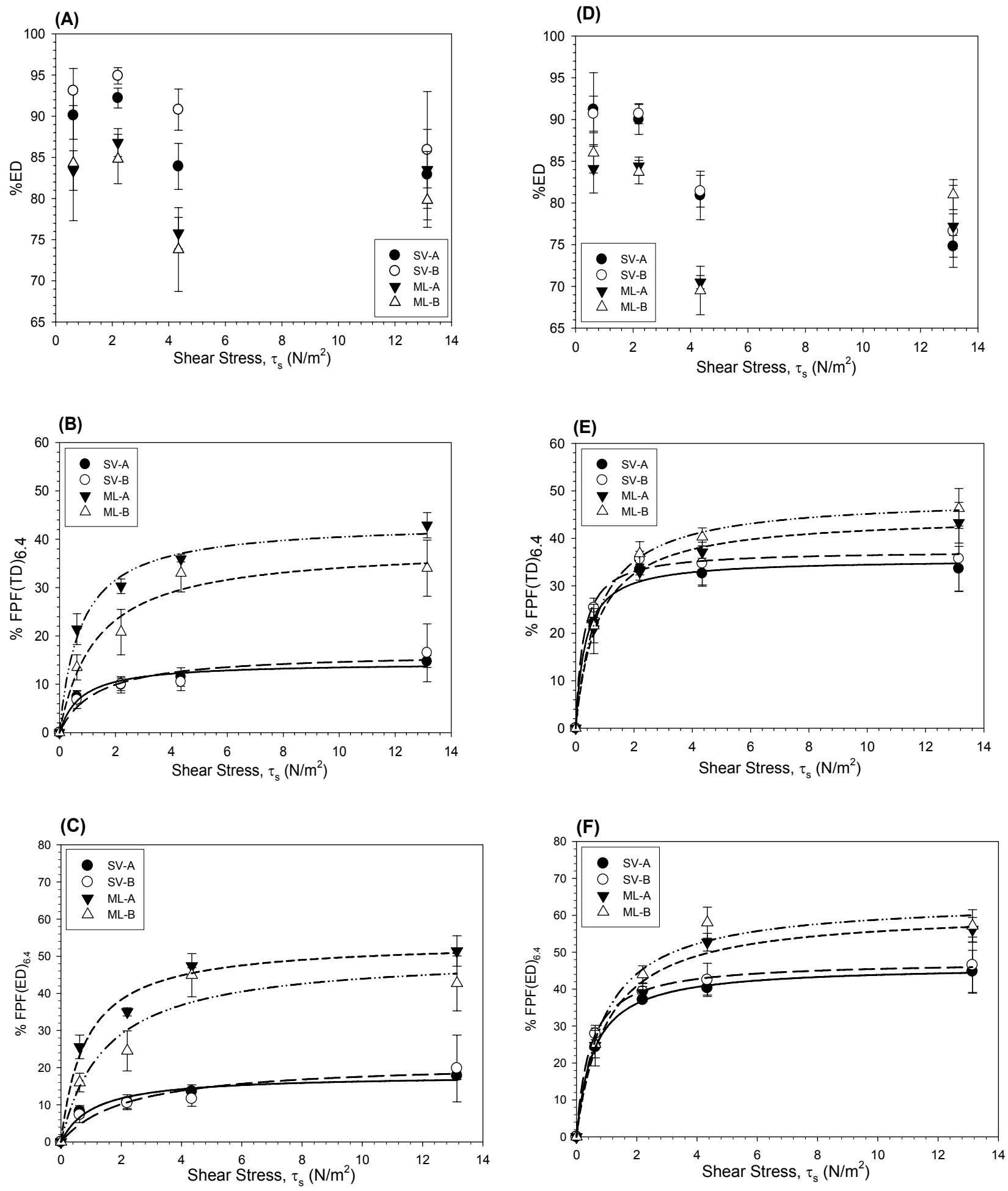
Figure 3-8. The relationship between SET airflow parameter (exemplified by shear stress $\tau_{\mathrm{s}}$ ) and TSLI formulation performance (exemplified by $F P F_{T D}$ ). Linear regression analyses were applied using Langmuir linear regression. (Eq. 3-2). (A) 2\% (w/w) AS/lactose monohydrate systems; and (B) 2\% (w/w) DSCG/lactose monohydrate systems. The $R^{2}$ represents the goodness of fit of regression lines. (Mean $\pm \mathrm{SD}, n=3$ ).

(A)

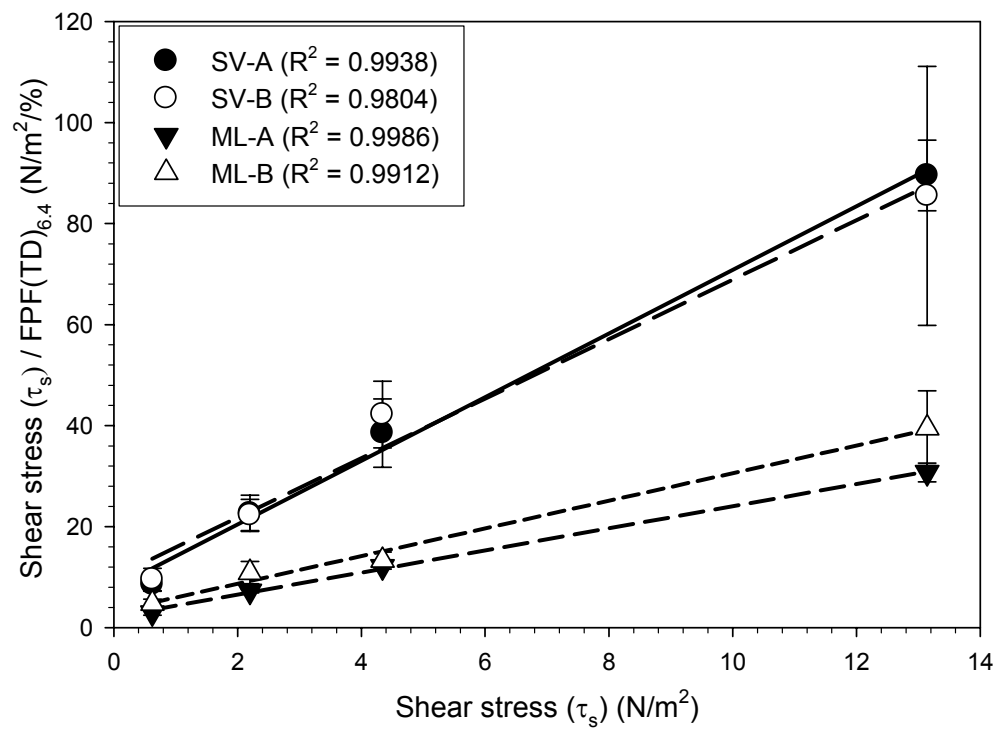

(B)

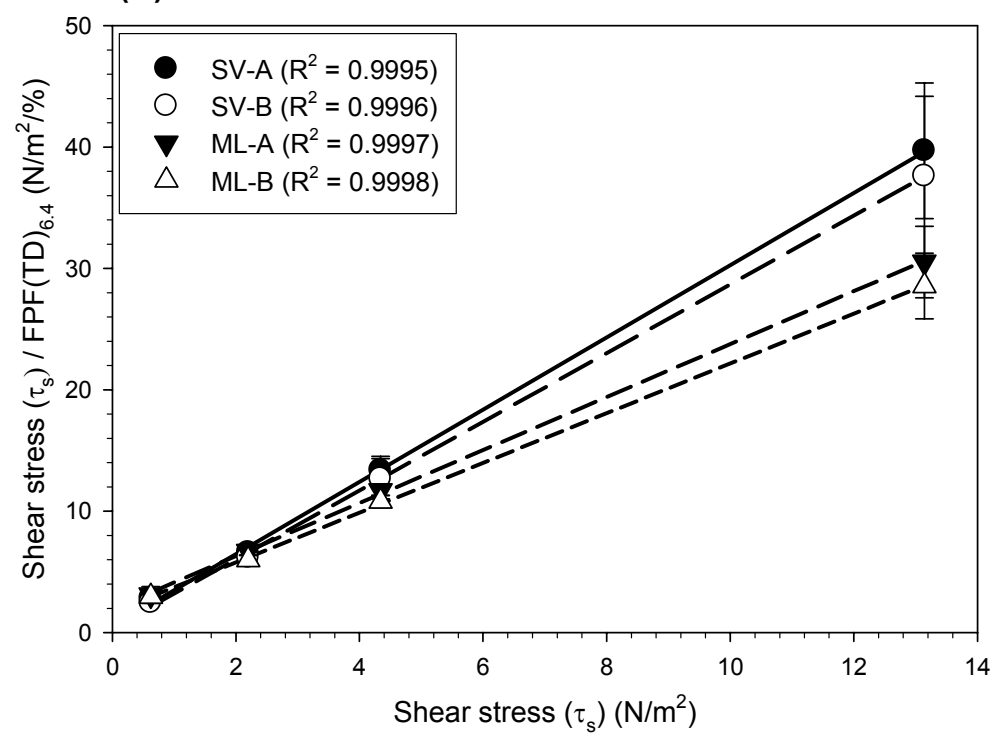


Figure 3-9. The relationship between SET shear stress $\left(\tau_{\mathrm{s}}\right)$ and TSLI formulation performance $\left(E D, F P F_{T D}, F P F_{E D}\right)$. Nonlinear regression analyses were applied using PADE for $F P F-\tau_{s}$ correlation (Eq.3-1). Left panels (A) to (C) are IPB/lactose monohydrate systems $(2 \% \mathrm{w} / \mathrm{w})$ : (A) $E D-\tau_{s}$; (B) $F P F_{T D^{-}} \tau_{s}$ curve fitting; (C) $F P F_{E D^{-}} \tau_{s}$ curve fitting. Right panels (D) to (F) are FP/lactose monohydrate systems ( $2 \% \mathrm{w} / \mathrm{w}):(D)$ $E D-\tau_{s}$; (E) $F P F_{T D^{-}} \tau_{S}$ curve fitting; (F) $F P F_{E D^{-}} \tau_{S}$ curve fitting. (Mean $\pm \mathrm{SD}, n=3$ ).
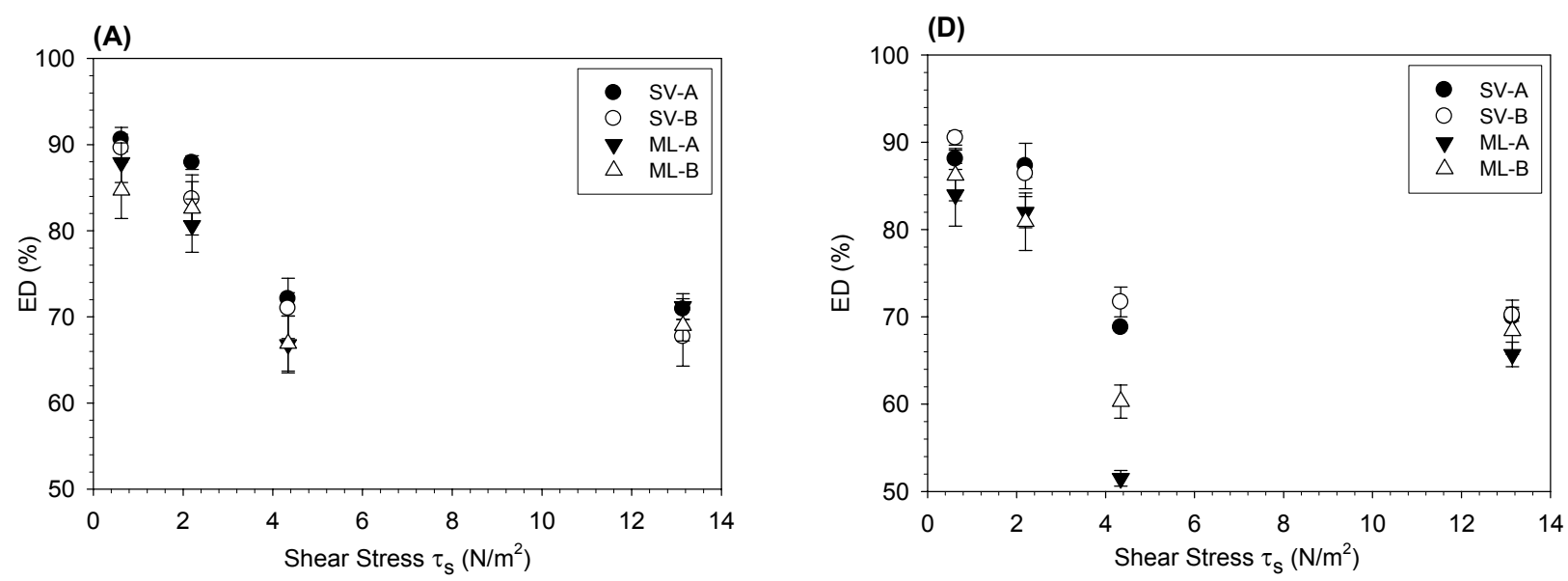

(B)

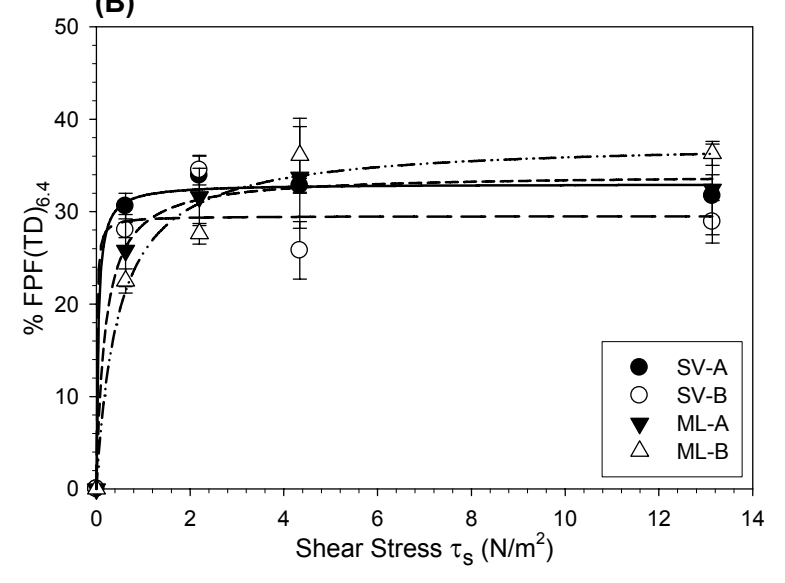

(C)

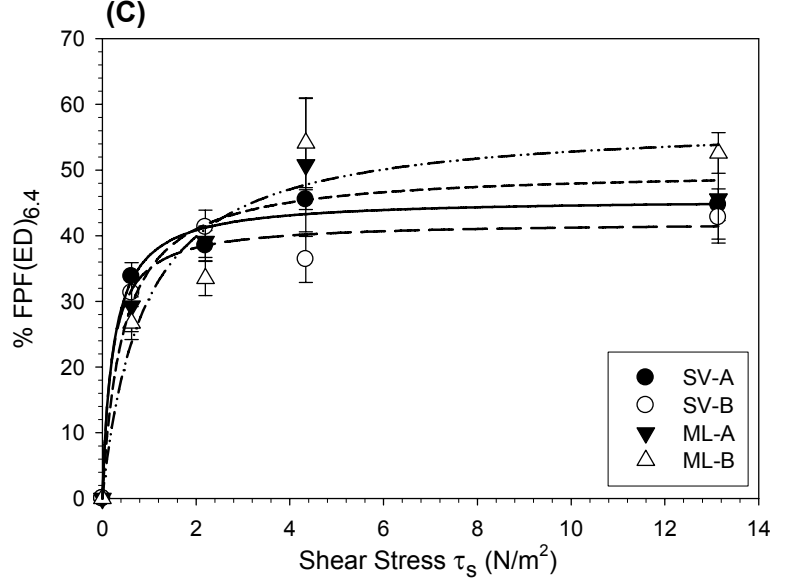

(E)

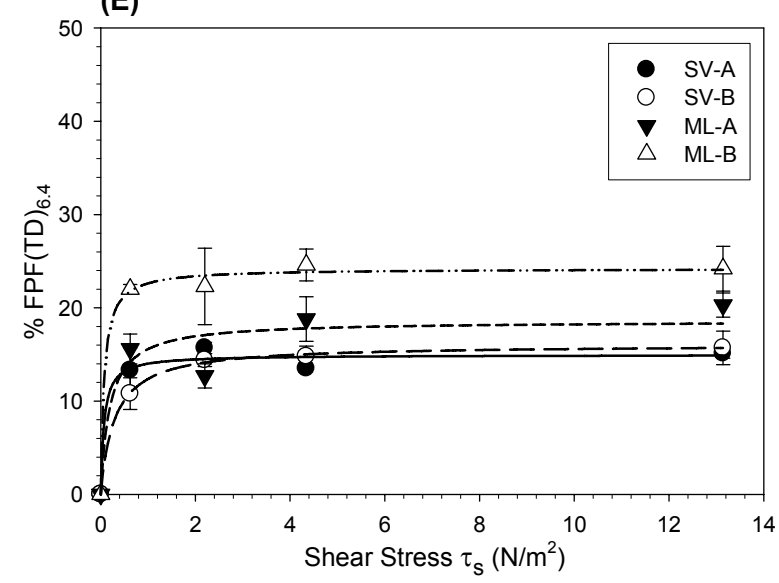

(F)

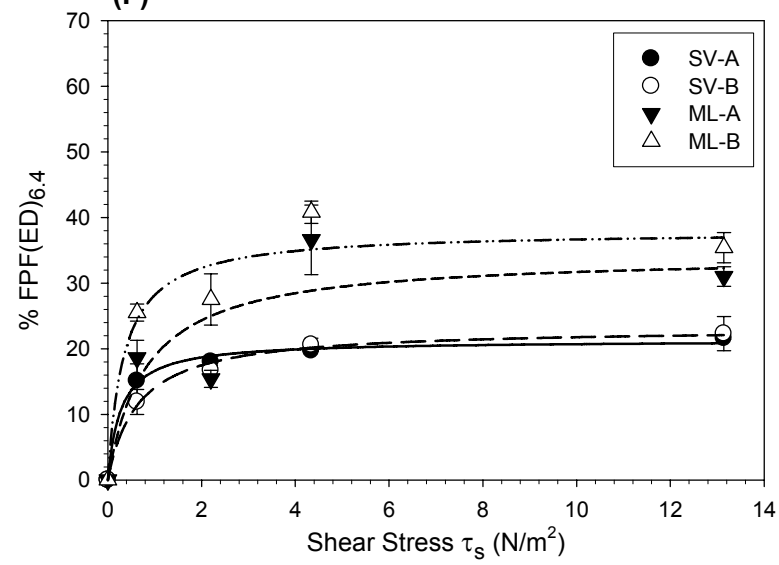


Figure 3-10. The relationship between TSLI formulation performance (exemplified by $\left.F P F_{T D}\right)$ and SET shear stress $\left(\tau_{s}\right)$. Linear regression analyses were applied using PADE linear regression (Eq. 3-2). (A) $2 \% \mathrm{w} / \mathrm{w}$ IPB/lactose monohydrate systems; and (B) $2 \% \mathrm{w} / \mathrm{w}$ FP/lactose monohydrate systems. The $R^{2}$ represents the goodness of fit of regression lines. (Mean $\pm \mathrm{SD}, n=3$ ).

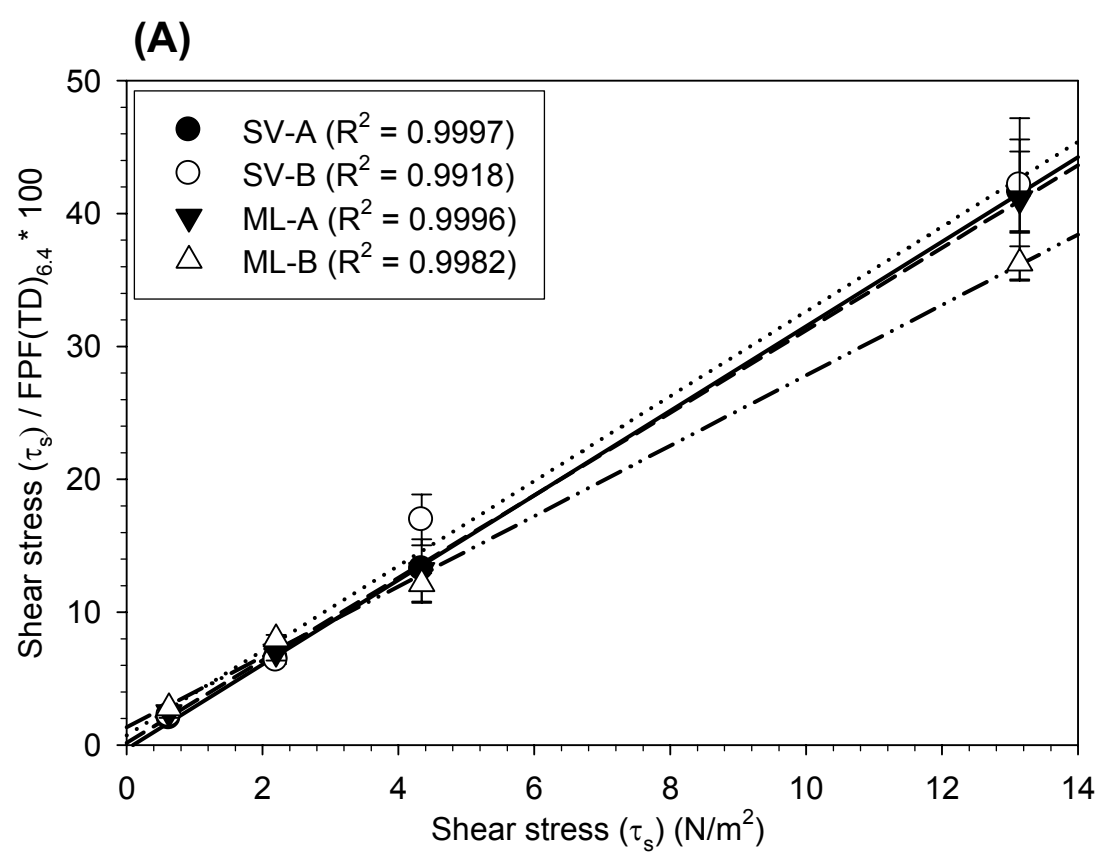

(B)

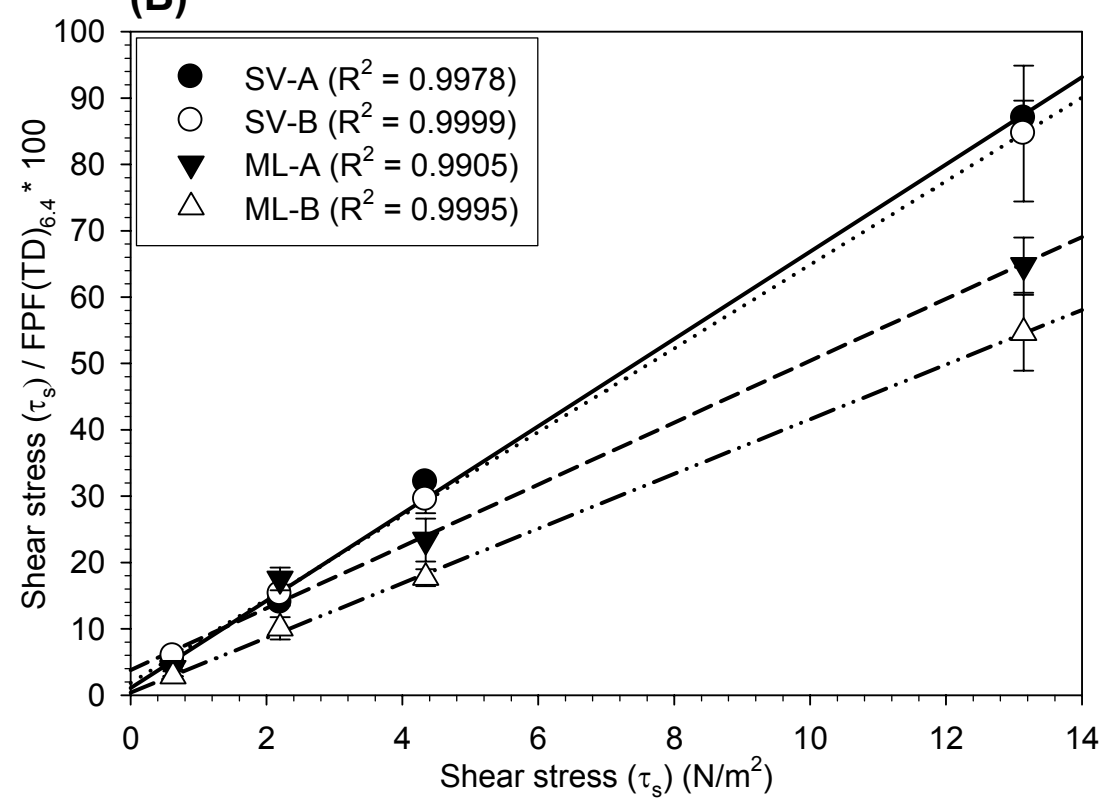


Figure 3-11. The ACI deposition pattern of AS/SV, DSCG/ML, IPB/SV, and FP/ML formulations using SETs (Device, throat, and preseparator drug deposition were included in stage deposition calculation but not plotted). (A) $2 \%(\mathrm{w} / \mathrm{w}) \mathrm{AS} / \mathrm{SV}$ at lowest shear stress (SET-D, $\left.\tau_{s}=0.624 \mathrm{~N} / \mathrm{m}^{2}\right)$ vs. $2 \%(\mathrm{w} / \mathrm{w}) \mathrm{DSCG} / \mathrm{ML}$ at highest shear stress (SET-A, $\left.\tau_{s}=13.143 \mathrm{~N} / \mathrm{m}^{2}\right)$; (B) $2 \%(\mathrm{w} / \mathrm{w}) \mathrm{AS} / \mathrm{SV}$ at highest shear stress $v s .2 \%(\mathrm{w} / \mathrm{w}) \mathrm{DSCG} / \mathrm{ML}$ at lowest shear stress (SET-A, $\left.\tau_{s}=13.143 \mathrm{~N} / \mathrm{m}^{2}\right)$; (C) $2 \%(\mathrm{w} / \mathrm{w}) \mathrm{IPB} / \mathrm{SV}$ at lowest shear stress vs. 2\% (w/w) FP/ML at highest shear stress; (D) 2\% (w/w) IPB/SV at highest shear stress $v s .2 \%(\mathrm{w} / \mathrm{w}) \mathrm{FP} / \mathrm{ML}$ at lowest shear stress. (Mean $\pm \mathrm{SD}, n=3$ ).

(A)

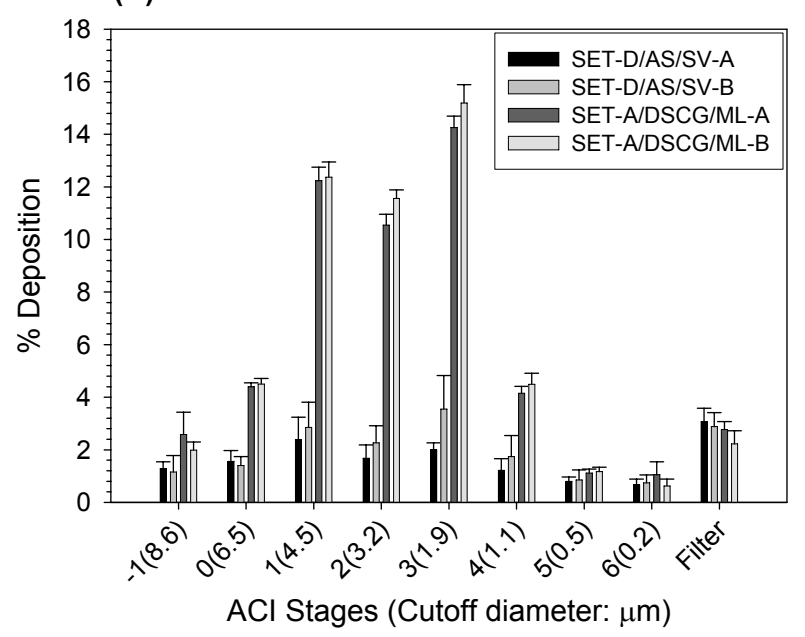

(C)

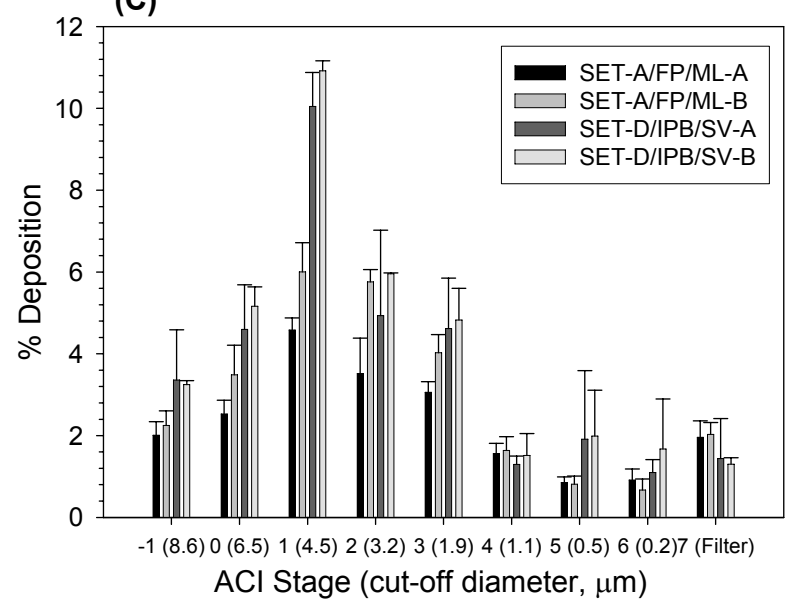

(B)
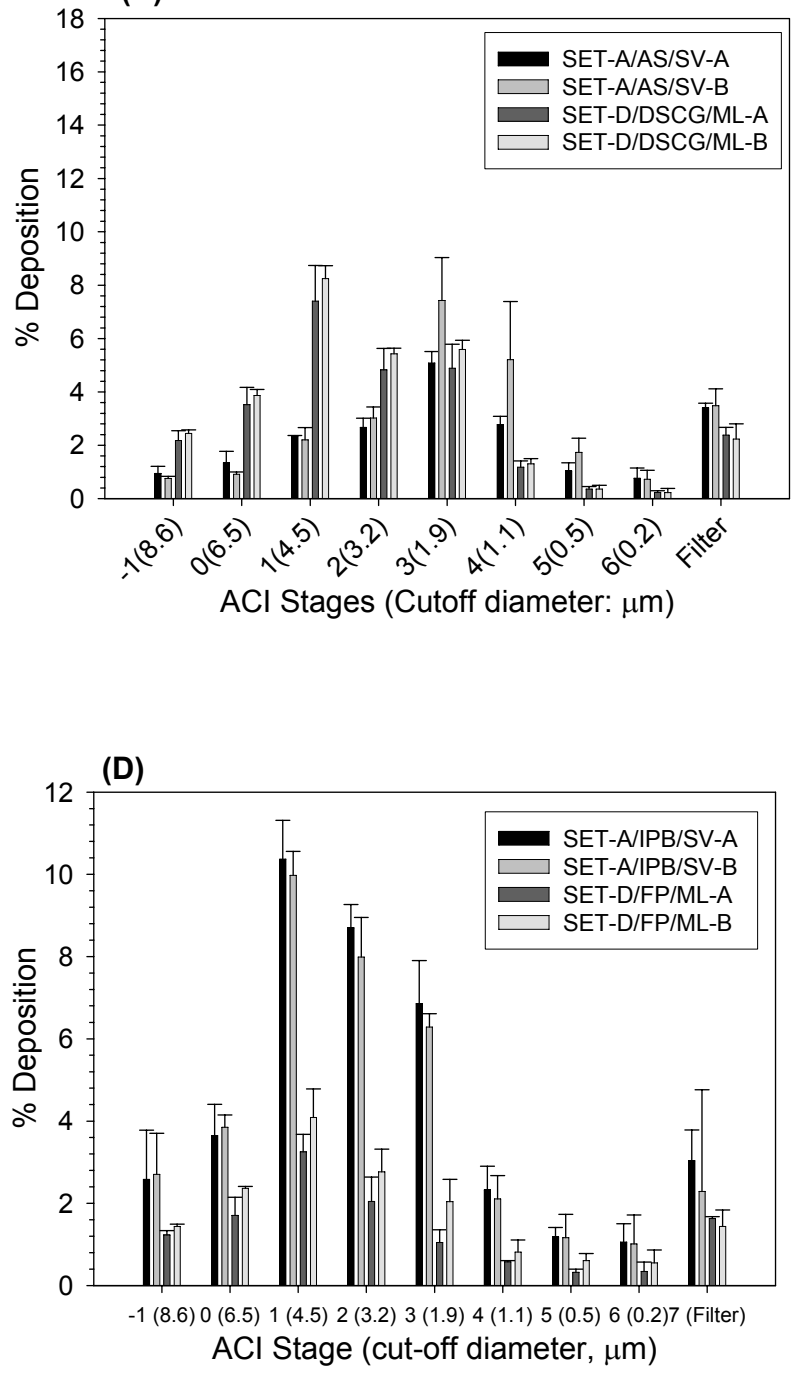
Figure 3-12. ACI Performance Evaluation $\left(F P F_{T D<6.5}\right)$ by Dual-point Performance Evaluation, FPF and shear stress (SET-D, $\tau_{s}=0.624 \mathrm{~N} / \mathrm{m}^{2}$ and SET-A, $\tau_{s}=13.143 \mathrm{~N} / \mathrm{m}^{2}$ ) correlation: (A) 2\% (w/w) AS/SV vs. 2\% (w/w) DSCG/ML; and (B) 2\% (w/w) IPB/SV vs. $2 \%(\mathrm{w} / \mathrm{w}) \mathrm{FP} / \mathrm{ML} \quad($ Mean $\pm \mathrm{SD}, n=3)$.

(A)

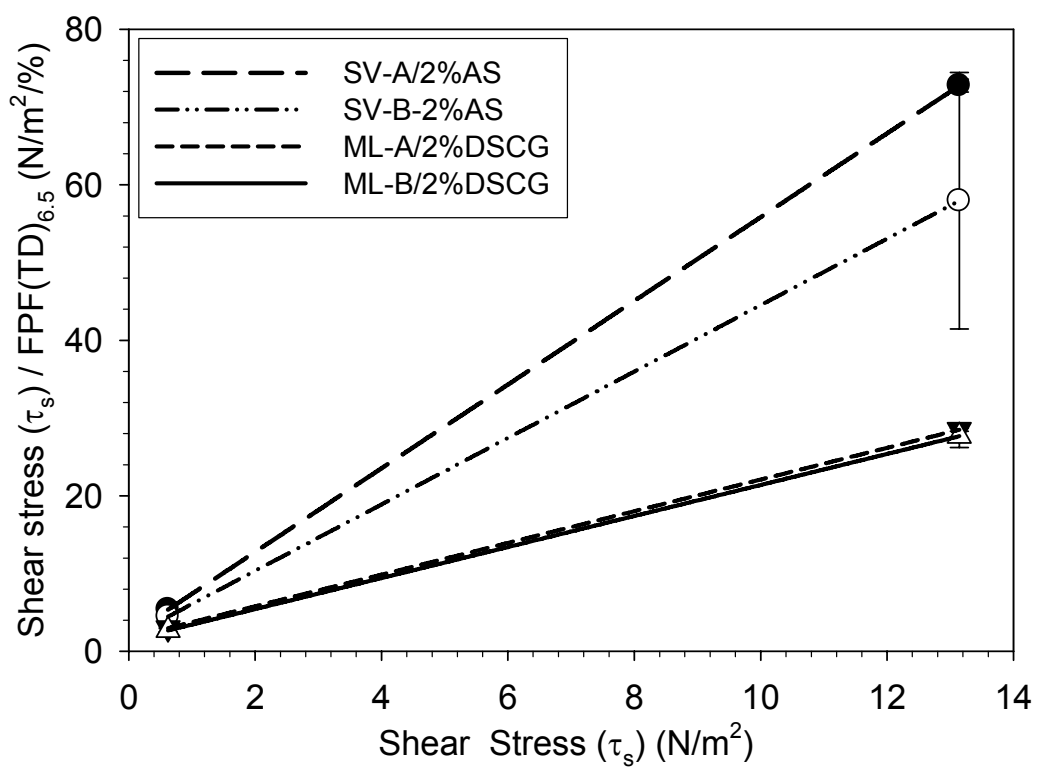

(B)

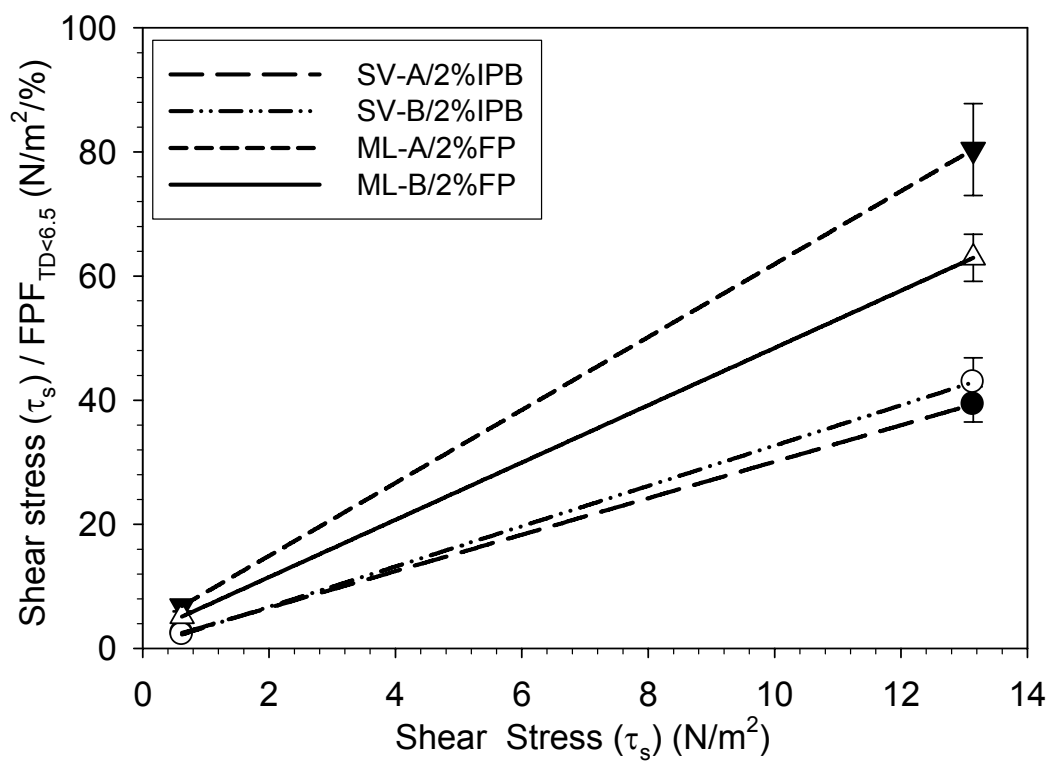




\section{References}

1. V.A. Cullum, J.B. Farmer, D. Jack and G.P. Levy, Br J Pharmacol, 35, 141-51 (1969).

2. A.J. Hickey, H.M. Mansour, M.J. Telko, Z. Xu, H.D. Smyth, T. Mulder, R. McLean, J. Langridge and D. Papadopoulos, J Pharm Sci, 96, 1302-1319 (2007).

3. J.S. Cox, Nature, 216, 1328-9 (1967).

4. M.K. Taylor, Particle engineering to achieve optimal morphology and physicochemical characteristics for aerosol delivery of ipratropium bromide, $\mathrm{Ph}$. D. Thesis, University of North Carolina, Chapel Hill (2001).

5. H. Kerstjens, D. Postma and N. ten Hacken, Clin Evid, 1923-47 (2005).

6. C. Crim, L.N. Pierre and P.T. Daley-Yates, Clin Ther, 23, 1339-54 (2001).

7. R. Newton, Thorax, 55, 603-613 (2000).

8. L.R. Chen, V.G. Young, Jr., D. Lechuga-Ballesteros and D.J. Grant, J Pharm Sci, 88, 1191-200 (1999).

9. J.S. Cox, G.D. Woodard and W.C. McCrone, J Pharm Sci, 60, 1458-65 (1971).

10. M.K. Taylor, A.J. Hickey and M. VanOort, Pharm Dev Technol, 11, 321-336 (2006).

11. J.M. van Zyl, B. Derendinger, H.I. Seifart and P. Van der Bijl, Int J Pharm, 357, 32-6 (2008).

12. A.P. Baptist and R.C. Reddy, J Clin Pharm Ther, 34, 1-12 (2009).

13. A.J. Hickey, H.M. Mansour, M.J. Telko, Z. Xu, H.D. Smyth, T. Mulder, R. McLean, J. Langridge and D. Papadopoulos, J Pharm Sci, 96, 1282-1301 (2007).

14. M.D. Louey, M. VanOort and A.J. Hickey, J. Aerosol Sci., 37, 1520-1533 (2006).

15. C.A. Dunbar, A.J. Hickey and H. P., Kona, 16, 7-45 (1998).

16. R. Westmeier and H. Steckel, J Pharm Sci, 97, 2299-2310 (2008).

17. M.D. Louey, M. Van Oort and A.J. Hickey, Pharm Res, 21, 1207-1213 (2004). 
18. H.G. Brittain and K. Florey, Profiles of drug substances, excipients and related methodology. Academic Press, (2003).

19. Cooper, Simon, Murray and G.W.R. Development, A novel polymorphic crystalline form of fluticasone propionate, a method for its production and pharmaceutical compositions thereofUK. (1998).

20. H. Smyth and A.J. Hickey, Am J Drug Deliv, 3, 117-132 (2005).

21. Z. Xu, H.M. Mansour, T. Mulder, R. McLean, J. Langridge and A.J. Hickey, $J$ Pharm Sci, (2010 in press).

22. Z. Xu, H.M. Mansour, T. Mulder, R. McLean, J. Langridge and A.J. Hickey, $J$ Pharm Sci, (2009 submitted).

23. D. Dollimore and P. Spooner, J Appl Chem Biotech, 24, 35-41 (1974).

24. P.M. Young, M.J. Tobyn, R. Price, M. Buttrum and F. Dey, J Pharm Sci, 95, 1800-1809 (2006).

25. D. Attwood and S.P. Agarwal, Int J Pharm, 22, 25-30 (1984).

26. X. Ding, T.C. Stringfellow and J.R. Robinson, J Pharm Sci, 93, 1351-8 (2004).

27. Z. Xu, H.M. Mansour, T. Mulder, R. McLean, J. Langridge and A.J. Hickey, Respiratory Drug Delivery, 3, 897-900 (2008).

28. M.D. Jones and R. Price, Pharm Res, 23, 1665-1674 (2006).

29. H. Adi, I. Larson, H. Chiou, P. Young, D. Traini and P. Stewart, J Pharm Sci, 97, 3140-52 (2008).

30. B. Beilmann, R. Kubiak, P. Grab, H. Hausler and P. Langguth, AAPS PharmSciTech, 8, Article 31 (2007).

31. M.D. Jones, J.C. Hooton, M.L. Dawson, A.R. Ferrie and R. Price, Pharm Res, 25, 337-348 (2008).

32. M.D. Louey and P.J. Stewart, Pharm Res, 19, 1524-1531 (2002).

33. X.M. Zeng, G.P. Martin and C. Marriott, Particulate Interactions in Dry Powder Formulations for Inhalation. Taylor \& Francis, New York (2001). 
34. A.H. de Boer, P. Hagedoorn, D. Gjaltema, J. Goede and H.W. Frijlink, Int J Pharm, 260, 187-200 (2003).

35. S.C. Nichols and E. Wynn, RDD 2008, 1, 175-184 (2008).

36. H.P. Dunbar CHA, Kona, 16, 7-45 (1998).

37. N. Islam and E. Gladki, Int J Pharm, 360, 1-11 (2008).

38. Z. Xu, H.M. Mansour, T. Mulder, R. McLean, J. Langridge and A.J. Hickey, $J$ Pharm Sci, (2009 submitted).

39. D.D. Do, Adsorption analysis: equilibria and kinetics. Imperial College Press, London, UK (1998). 


\section{CHAPTER 4 ALTERNATIVE SUGARS AS CARRIER}

\subsection{Introduction}

Although $\alpha$-lactose monohydrate is most widely used DPI carrier, the selection of this sugar carrier was not based on any rigorous screening process. Historically, lactose monohydrate has been commonly employed as an excipient in many solid and liquid dosage forms; because it exhibits several ideal characteristics, such as its safety profile, availability, and cost. However, there are quite a few other pharmaceutical excipients, especially sugars (defined as low molecular weight carbohydrate including mono-, di-, oligo-saccharides) and sugar alcohols, which may also meet the criteria of a good carrier. Sugars are a group of complex small molecules with condensed functionalities (polyol, aldehyde, ketone) and chiral centers. There are six naturally occurring pentoses (four aldo- and two keto-) and twelve hexose (eight aldo- and four keto-). In solution, they exist in both open chain aldehyde/ketone and cyclic hemiacetals. Mutarotation further complicates the chemistry. The sugar alcohols are a group of polyols derived from corresponding sugars. It should also be noted that in majority pharmaceutical dosage forms, both sugars and sugar alcohols are presented in their thermodynamically most stable form, many of which are hydrate crystallines.

A plethora of sugars and sugar alcohols exists but they were not sufficiently studied. The selection criteria were largely based on their availability and generally recognized as safe (GRAS) food additives. Their safety profiles for pulmonary delivery 
were not complete. The potential carrier sugar explored so far (Figure 4-1) include glucose [1-3], trehalose [4-6], maltose [2], raffinose [4, 5], and $\beta$-cyclodextrin $[4,5]$. The sugar alcohols include erythritol $[7,8]$, xylitol $[1,4,5]$, mannitol $[1,6,9,10]$, sorbitol $[2$, 9], and maltitol $[1,10]$. Physicochemical investigations of these sugars relating with the formulation performance have only been attributed to a limited number of aspects. They include: differences in particle size or shape [9], surface roughness [8, 10], surface energy $[6,10]$, drug carrier adhesion [7, 10], and hygroscopicity $[2,11]$. In fact, extra aspects should be considered carefully before switching to a new carrier system. The safety profiles of any alternative sugars should be under regulatory scrutiny. The long-term stability (physicochemical, pharmaceutical, and microbiological aspects) of final products containing the alternative sugars should be established at an early stage of product development. These concerns somehow delayed the development of alternative sugars into carriers. On the other hand, lactose as an inert carrier should come under some degree of scrutiny too. Lactose contains aldehyde functionality in its open chain form, its reducing characteristics may not be suitable for some active compounds such as peptides, proteins, or other amino-group containing compounds, non-enzymatic browning known as the Maillard reaction.[12] Reducing sugars also has the potential for microbes to use sugars as substrates for growth, adversely affect the anti-microbial drug therapy. Moreover, a proportion of people have a certain degree of lactose intolerance, which raises another issue for the lactose based formulations.[13] Lactose as a milk product, may introduce small amount of surface impurity during the manufacturing, which causes batch to batch 
variation of the final product. Endotoxin content and bovine source of lactose are also minor concerns of using lactose.[1]

The search for alternative sugar carriers based on all the above criteria is a daunting task. However, several simple rules that aim at limiting the scope of search may be enlightening. In chemistry, non-reducing sugars and sugar alcohols have the advantage of chemical stability and less likely being nutrient for bacterial growth. Some non-reducing sugar alcholos such as erythritol and xylitol cannot be metabolized and have the tendency to "starve" harmful bacteria.[14] In physics, an ideal sugar carrier should be non-hygroscopic (high critical relative humidity, $\mathrm{RH}_{0}$ ), stable during pharmaceutical processing and storage (e.g. high glass transition temperature), relative smooth surface crystalline without metastable structures and polymorphs. Molecules such as fructose and sorbitol $\left(\mathrm{RH}_{0}=64 \%\right.$ and $70 \%$, respectively, at $\left.25{ }^{\circ} \mathrm{C}[15]\right)$ are examples of hygroscopic sugar and sugar alcohol that may not be suitable carrier candidates. Based on these physicochemical criteria, a representative non-reducing sugar D-trehalose dihydrate, and sugar alcohol D-mannitol were chosen as potential carriers for the pulmonary drug delivery. The former is a disaccharide formed by 1,1-glucoside linkage, which makes the molecule resistant to acid hydrolysis and remains in closed-ring form (Acetal is non-reducing). Trehalose is accepted as a food ingredient under the GRAS terms in the U.S. It is manufactured by enzymatic hydrolysis of wheat or corn starch.[16] Because of its effect on stabilizing macromolecules such as vaccines, it is a potential excipient as a non-toxic cryoprotectant.[17] Anhydrous D-trehalose has relatively high a glass transition temperature at $115{ }^{\circ} \mathrm{C}$.[17] The crystalline D-trehalose dihydrate has both a high 
resistance to moisture $\left(\mathrm{RH}_{0} \sim 98 \%\right.$ at $\left.23{ }^{\circ} \mathrm{C}[15]\right)$ and absorption and a characteristic of retaining high Young's modulus (the force per unit area required to break or crack a substance) at high relative humidity.[18] The latter, D-mannitol is a six carbon sugar alcohol. It is often used as a stabilizing agent for proteins and peptides. It has been used as a therapeutic to treat head trauma and oliguric renal failure. The inhalation mannitol has been used as a treatment for cystic fibrosis (to osmotically thin the mucus) and bronchiectasis (Bronchitol ${ }^{\mathrm{TM}}$ ) and as a diagnostic test for airway hyperresponsiveness $\left(\right.$ Aridol $\left.^{\mathrm{TM}}\right)$. Because mannitol is metabolized independently of insulin, it is applicable in diabetic patient. At present, mannitol is manufactured by chemical hydrogenation of fructose or glucose.[19] Other methods such as enzymatic hydrogenation and microbial production are also used but more costly. Crystalline D-mannitol is a relatively soft material (Young's modulus: 12.2 GPa [20]). Crystalline D-mannitol $\left(\mathrm{RH}_{0} \sim 80 \%\right.$ at $25{ }^{\circ} \mathrm{C}$ [15]) is more hygroscopic than D-trehalose dihydrate.

As described in previous chapters and publications [21-26] using lactose-based formulations, various interparticulate and interfacial interactions may greatly affect drug aerosolization performance. The influencing factors including particle size, size distribution, morphology, surface roughness, degree of crystallinity vs. noncrystallinity, the presence of metastable surface phases by pharmaceutical processing, surface energetics, elasticity, electrostatic interactions surface heterogeneity of drugs and carriers should apply to the potential sugar carriers too. For example, it was reported previous that different qualities of mannitol batches (Sieved fractions: 32-125 $\mu \mathrm{m}$ ) resulted in significantly different FPF of budesonide, varying from 15-50\%.[1] Based on the earlier 
studies using standardized entrainment tubes (SETs) and aerodynamic deposition studies for the evaluation of aerosolization performance, we have obtained valuable information about the physicochemical behavior of drugs, different batches of lactose monohydrate carriers, and their corresponding blend formulations. The discovery of the novel powder aerosol deaggregation equation (PADE) greatly broadened our horizon of knowledge for evaluating performance in a shear stress range. However, it is still unknown if the aerosolization performance using alternative sugars would furnish similar result that could apply PADE. The purpose of the studies in this chapter is to comprehensively characterize the physicochemical properties, to systematically generate performance data using SETs and inertial sampling, and to investigate the robustness and predictive behavior of PADE method in alternative carriers.

\subsection{Material and Methods}

\subsubsection{Material}

The pulmonary drugs are the same as those described in Chapter 3. They include micronized albuterol sulfate (AS, $\mathrm{C}_{13} \mathrm{H}_{23} \mathrm{NO}_{7} \mathrm{~S}$; MW $337.39 \mathrm{~g} / \mathrm{mol}$; Pfizer Global R\&D, Kent, UK); disodium cromoglycate $\left(\mathrm{C}_{23} \mathrm{H}_{14} \mathrm{Na}_{2} \mathrm{O}_{11}\right.$; Molecular Weight: $512.33 \mathrm{~g} / \mathrm{mol}$; Sigma-Aldrich Inc, St. Louis, MO, US); ipratropium bromide monohydrate $\left(\mathrm{C}_{20} \mathrm{H}_{31} \mathrm{Br}_{2} \mathrm{NO}_{4}\right.$; Molecular Weight: $509.27 \mathrm{~g} / \mathrm{mol}$; Sigma-Aldrich Inc., St. Louis, MO, US); and fluticasone propionate $\left(\mathrm{C}_{25} \mathrm{H}_{31} \mathrm{~F}_{3} \mathrm{O}_{5} \mathrm{~S}\right.$; Molecular Weight: $500.57 \mathrm{~g} / \mathrm{mol}$; API Inc., P.R. China). The micronization process was the same as that described in Chapter 3. 
Carriers used were D-mannitol $\left(\mathrm{C}_{6} \mathrm{H}_{14} \mathrm{O}_{6}\right.$; Molecular Weight: $182.17 \mathrm{~g} / \mathrm{mol}$; ACS reagent, Batch No: 047K0064, Sigma-Aldrich Inc., St. Louis, MO, US) and D-(+)-trehalose dihydrate $\left(\mathrm{C}_{12} \mathrm{H}_{26} \mathrm{O}_{13}\right.$; Molecular Weight: $378.33 \mathrm{~g} / \mathrm{mol} ; \geq 98.5 \%$ purity, Batch No.: 047K3779, Sigma-Aldrich Inc., St. Louis, MO, US). Both carriers were sieved. The sieving was operated in a dry box with relative humidity controlled $(\sim 20 \% \mathrm{RH}, 23 \pm$ $\left.0.5{ }^{\circ} \mathrm{C}\right)$. The sieve mesh sizes of $45,75,125,180$, and $300 \mu \mathrm{m}$ were placed on a 3 -inch sieve shaker (Model No. SS-5, Gilson Company Inc. Worthington, OH). The sieves were arranged in descending order of mesh size with the $300 \mu \mathrm{m}$ sieve on top and the $45 \mu \mathrm{m}$ sieve on the bottom. A mass of about $10 \mathrm{~g}$ of powder were placed on the top sieve and the sieve shaker was operated in the tap mode for two hours. Particles in the 0-45 $\mu \mathrm{m}, 45-75$ $\mu \mathrm{m}$, and 75-125 $\mu \mathrm{m}$ size ranges were collected. The sieved fractions $(2$ sugars $\times 3$ fractions) were stored in sealed glass jars (Wheaton Science Products, Millville, NJ) which were then stored in glass desiccators under ambient temperature and pressure prior to further characterization and blending.

The carrier particle sizes in the range of 45-75 $\mu \mathrm{m}$ were used for preparation of physical interactive blends. The 4 drugs $\times 2$ carriers were prepared by the same process as that described in Chapter $\mathbf{2}$ and 3. The prepared blends were stored in glass desiccators at ambient condition prior to further characterization and aerosolization performance study.

\subsubsection{Analytical Approaches}

The same physical characterization including scanning electron microscopy (SEM), thermal analysis using differential scanning calorimetry (DSC), crystallinity using X-ray 
powder diffraction (XRPD), and particle sizing using laser diffraction (LD) (100 mm focal length for carrier particle size analysis) were employed. (see Chapter $\mathbf{3}$ for detailed operation).

\section{$\underline{4.2 .3 \text { In Vitro Aerosol Performance Characterization }}$}

Similar aerosolization using four SETs (SET-A, B, C, and D) and TSLI was employed. (see Chapter 3 for detailed method). The aerodynamic performance was evaluated by emitted dose (ED), fine particle fraction from total dose $\left(\mathrm{FPF}_{\mathrm{TD}}\right)$ and from emitted dose $\left(\mathrm{FPF}_{\mathrm{ED}}\right.$ ). SigmaPlot 9.01 (Jandell Scientific, Systat software, San Jose, CA) was used for all data analysis and statistics. The PADE nonlinear (Eq. 4-1) and PADE linear (Eq. 4-2) regression analyses, as the algebraic equivalent expression of Langmuir surface adsorption theory, were applied.

$$
\begin{gathered}
\frac{F P F}{F P F_{\max }}=\frac{k_{d} \tau_{s}}{1+k_{d} \tau_{s}} \\
\frac{\tau_{s}}{F P F}=\frac{\tau_{s}}{F P F_{\text {max }}}+\frac{1}{k_{d}\left(F P F_{\text {max }}\right)}
\end{gathered}
$$

\subsection{Results}

\subsubsection{Aerosol Characterization}

4.3.1.1 Particle size and morphology (SEM) 
Representative SEM images are shown in Figure 4-2 and 4-3 for sieved D-mannitol (Mann), sieved D-trehalose dihydrate (Treh) (both 45-75 sieve range) and their interactive physical mixtures with four micronized pulmonary drugs. The SEM images of the drugs alone including albuterol sulfate (AS), disodium cromoglycate (DSCG), ipratropium bromide monohydrate (IPB), and fluticasone propionate (FP) were presented in Figure 2-3 and 3-2. Treh (Figure 4-2A) exhibited columnar shape and very smooth crystalline surfaces with few surface fractures that were perpendicular to the length of the crystal. The surface fractures were possibly induced by mechanical sieving because the raw material SEM did not exhibit these fractures. Almost no intrinsic surface fine particles were observed. Conversely, Mann (Figure 4-2B) exhibited rough surface and long fractures along the length of the particle surface. The intrinsic fine particles with irregular shapes were resided in the grooves of primary particle surfaces (mechanical interlocking) where surface discontinuities exist even after sieving. The SEM of physical interactive mixtures demonstrated homogenous distribution of drug particles on the surface of the primary carrier particles for all the mixtures. Very few drug agglomerations were observed in the void of primary particles.

\subsubsection{Thermal properties (DSC)}

Representative DSC thermograms of Mann and Treh at a heating rate of $5{ }^{\circ} \mathrm{C} / \mathrm{min}$ are shown in Figure 4-4. The DSC thermograms of drugs (unmilled and micronized) were shown in Figure 3-4 and discussed in Chapter 3. As seen in Figure 4-4A, Mann exhibited a single endothermic heat of fusing $\left(\mathrm{T}_{\mathrm{m}} \sim 168{ }^{\circ} \mathrm{C}\right.$ and $\left.\Delta \mathrm{H} \sim 264 \mathrm{~J} / \mathrm{g}\right)$. In Figure 
4-4B, Treh exhibited an endothermic dehydration peak $\left(\mathrm{T}_{\mathrm{deh}} \sim 93{ }^{\circ} \mathrm{C}\right.$ and $\left.\Delta \mathrm{H}_{1} \sim 259 \mathrm{~J} / \mathrm{g}\right)$ followed by a smaller endothermic heat of fusing $\left(\mathrm{T}_{\mathrm{m}} \sim 212{ }^{\circ} \mathrm{C}\right.$ and $\left.\Delta \mathrm{H}_{2} \sim 112 \mathrm{~J} / \mathrm{g}\right)$ in the first scan. A small exothermic peak $\left(\mathrm{T}_{\text {rec }} \sim 155^{\circ} \mathrm{C}\right.$ and $\left.\Delta \mathrm{H}_{3} \sim-15 \mathrm{~J} / \mathrm{g}\right)$ was observed between the two endothermic peaks, indicating the molecular disorder to ordered state transition (recrystallization). Treh also exhibited scanning rate dependency. At scanning rate of $10{ }^{\circ} \mathrm{C} / \mathrm{min}$, the single dehydration peak split into doublet. Decomposition of Treh was observed when temperature was higher than $250{ }^{\circ} \mathrm{C}$. No glass transition $\left(\mathrm{T}_{\mathrm{g}}\right)$ was observed in the entire scanning range. However, by cooling the sample back to $20{ }^{\circ} \mathrm{C}$, a second scanning of the same sample from 25 to $300{ }^{\circ} \mathrm{C}$ at a scanning rate of $20{ }^{\circ} \mathrm{C} / \mathrm{min}$ exhibited an obvious $\mathrm{T}_{\mathrm{g}} \sim 115^{\circ} \mathrm{C}$ and $\Delta \mathrm{Cp} \sim 0.436 \mathrm{~J} /\left(\mathrm{g}{ }^{\circ} \mathrm{C}\right.$ ) (Figure 4-4B), indicating the glass transition of the amorphous D-trehalose caused by dehydration and cooling effect.

For AS/Treh formulation (Figure 4-5B), the dehydration endothermic peak of Treh $\left(\mathrm{T}_{\text {deh }} \sim 99^{\circ} \mathrm{C}\right.$ and $\left.\Delta \mathrm{H}_{2} \sim 250 \mathrm{~J} / \mathrm{g}\right)$ was comparable to that of Treh alone at a scanning rate of $5{ }^{\circ} \mathrm{C} / \mathrm{min}$. Two smaller endothermic peaks at $\sim 210$ and $220{ }^{\circ} \mathrm{C}$ were observed. The exothermic peak was shifted and partially overlapped with one of the small endothermic peak. For DSCG/Treh (Figure 4-5D), IPB/Treh (Figure 4-5F), FP/Treh (Figure 4-5H) formulations, the exothermic peak shifted to $\sim 180{ }^{\circ} \mathrm{C}$ and became more appreciable (broader and larger peak area) in all formulations. These changes in thermal properties of sugar carriers, similar to lactose monohydrate based formulations, may affect the molecular properties of the drugs and carriers which consequently can affect macroscopic aerosolization performance. 
The XRPD of Mann and Treh are shown in Figure 4-6A and B, respectively (Raw data: courtesy of Dr. Heidi Mansour. The data were re-plotted using Match!® software). Both diffractograms showed sharp intense peaks (with the absence of a noncrystalline "halo") characteristic of highly crystalline materials.[27] These are in good agreement with diffractograms reported in the University of Cambridge database.

\subsubsection{Particle size analysis (LD)}

The particle volume size distribution of micronized pulmonary drugs was shown in Chapter 3. The particle size distributions of Mann and Treh are shown Table 4-1. Mann had $\mathrm{D}_{50}$ of $52.95 \pm 1.69 \mu \mathrm{m}$ and span of $1.19 \pm 0.02$; and Treh had a $\mathrm{D}_{50}$ of $53.49 \pm 0.79$ $\mu \mathrm{m}$ and span of $0.84 \pm 0.04$. It indicated that the particle size distributions of both sieved Mann and Treh at a range of $45-75 \mu \mathrm{m}$ were comparable. A slightly larger span for Mann indicated that there were a few surface fines that could not be removed by mechanical sieving possibly due to mechanical interlocking. The LD data was in agreement with the SEM result.

\subsubsection{In Vitro Aeorsol Performance Assessment}

\subsubsection{Twin Stage Liquid Impinger (TSLI)}

A total of eight formulations ( 4 drugs $\times 2$ carriers) were subjected to aerosolization performance evaluation using four standardized entrainment tubes (SETs). The TSLI performance data are summarized in Appendix 4-A. In Chapter 3, a novel interpretation using powder aerosol deaggregation equation (PADE) was applied to describe and compare the particulate surface dissociation across a range of shear stress. Similar 
statistical analytical process was applied to the performance data generated using alternative carrier systems.

As seen in Figure 4-7, when SET shear stress $\left(\tau_{s}\right)$ was correlated with aerosolization performance data (demonstrated using $\mathrm{FPF}_{\mathrm{TD}<6.4}$ ), hyperbolic profiles were indicated. The non-linear regression analysis result (presented in $F P F_{\max }$ and $k_{d}$, see Chapter $\mathbf{3}$ and $\mathbf{5}$ for detailed description of these two parameters) and the regression goodness of fit (presented in $R^{2}$ and adjusted $R^{2}$ ) using Langmuir type PADE (Eq. 4-1) are shown in Table 4-2. The non-linear regression using $\mathrm{FPF}_{\mathrm{TD}}$ data and $\mathrm{FPF}_{\mathrm{ED}}$ data indicated good to excellent goodness of fit. The $R^{2}$ and adjusted $R^{2}$ values for all the evaluated formulations were in the ranges $0.8019-0.9845$ and $0.7359-0.9793$, respectively. (for $\mathrm{FPF}_{\mathrm{TD}}$ data). The rank order indicated by the value of $F P F_{\max }$ can be used to evaluate the performance efficiency and aid in formulation design. The evaluation of PADE linear regression indicated excellent correlation. The $R^{2}$ values ranged from 0.9274 to 0.9968 for the eight formulations investigated.

Drug Effect (AS, DSCG, IPB, and FP). The AS and DSCG formulations gave significantly higher $E D$ values than the other formulations, and FP formulation generally resulted in much lower $E D$ values. According to the value of $F P F_{\max }$, the performance efficiency (from $F P F_{T D}$ data) of Mann-based formulation gave a rank order of:

$$
\text { DSCG/Mann > FP/Mann > IPB/Mann > AS/Mann }
$$

The performance efficiency of Treh-based formulation gave a rank order of:

$$
\text { DSCG/Treh }>\text { IPB/Treh }>\text { AS/Treh }>\text { FP/Treh }
$$


Carrier Effect (Mann vs. Treh). The performance evaluated from $E D$ showed that carrier had limited influence on the $E D$. The $E D$ of Treh/IPB was higher than Mann/IPB (Figure 4-9). All the other formulations showed that the carrier effect was not significant. However, the performance efficiency (from $F P F_{T D}$ data) of
AS formulation gave a rank order of:
AS/Treh $>$ AS/Mann
DSCG formulation gave a rank order of:
DSCG/Mann > DSCG/Treh
IPB formulation gave a rank order of:
IPB/Treh > IPB/Mann
FP formulation gave a rank order of:
FP/Mann $>$ FP/Treh

SET Effect (low shear vs. high shear). Similar to the performance studies using SETs in Chapter $\mathbf{2}$ and 3, there was a general trend that higher shear stress values corresponded to lower $E D$ for all studied formulations. The explanation was that there existed two portions of entrained powder when using SETs. One portion involved particle deaggregation and the other did not (see Chapter 3 for detailed explanation).

\subsection{Discussion}

\section{$\underline{4.4 .1 \text { Influence of Drugs }}$}

The physicochemical nature of drugs is important to the aerosolization performance efficiency. The SEM images showed that after blending, all drugs were distributed homogeneously on the surface of the carrier particles. The particle size analysis indicated 
that the particle size distributions were comparable for all four drugs. Yet, DSCG formulations gave superior FPF values to the other formulations. The superior FPF values obtained of DSCG with both Treh and Mann formulations may be attributed to the strong interfacial energy-reducing properties. The unique physicochemical properties of DSCG [28] have been discussed in Chapter 3 when lactose monohydrate-based formulations were evaluated. This surface active drug was known to significantly lower the surface tension of high-energy surfaces such as water and form stable soluble monolayers at air-water interface.[29]

\subsubsection{Influence of Carriers}

Both Treh and Mann had similar particle size distribution after they were sieved. Although Treh and Mann had very different surface roughness and morphology, their influence on the aerosolization performance was not remarkable. Treh as carrier gave higher performance efficiency (indicated by $F P F_{\max }$ ) than Mann as carrier in AS and IPB systems, whereas Mann as carrier gave higher performance than Treh as carrier in the DSCG and FP systems. DSC thermogram of Treh indicated that it has a low dehydration temperature $\left(\sim 93{ }^{\circ} \mathrm{C}\right)$, caused by the loss of one or two bound water. At such a low dehydration temperature, the bound water loss could occur at low relative humidity and ambient temperature. Jones et al studied the dehydration of Treh and they showed that significant and irreversible changes in surface morphology occurred upon partial dehydration, in particular the formation of cracks.[30] This indicates that the surface properties of Treh may change during storage, which may occasionally favor but in most cases deteriorate the formulation performance efficiency, even though anhydrous Treh 
had a relatively high glass transition temperature. In this sense, the Treh formulations may be good candidate for the evaluation of formulation performance upon storage.

\subsubsection{Influence of SET shear stress}

For all the Treh and Mann-based aerosol systems presented in this study, the novel PADE method that allowed performance evaluation in the defined shear stress range was also applicable in the formulation using non-lactose sugar carriers. The PADE was developed based on the fundamental understanding that the forces acting at the particle interface are analogous to those at the molecular level, and that models of molecular surface association described by an adsorption expression can be adapted to fit shear displacement observations. As a shear force applies to the surface of a drug-coated carrier particle, no matter what the carrier is (the carrier could be lactose monohydrate, trehalose dihydrate or mannitol), the drug particles will be removed with increasing difficulty because of the sites that they occupy until a saturation is reached, when no drug particle can be removed at increasing shear force. The application of PADE using an algebraic equivalent expression to the Langmuir adsorption isotherm indicated that this novel method tolerated surface heterogeneity of different carriers and drugs. The heterogeneity refers to geometric features (such as surface roughness, morphology, polymorphism, and elasticity) and energetic features (surface energy distribution) and the drug-carrier interactions.

For all formulation systems studied so far (including lactose monohydrate based

formulations, Chapter 3), there existed a variant (shear stress-dependent) region at 
relatively low shear stress values and an invariant (shear stress-independent) region at higher shear stress range where the value of $F P F$ was independent with increasing shear stress values.[27] From the invariant region of the aerosolization profile, the $F P F_{\max }$ could be obtained, which has important predictive implications for formulation screening and rational design of optimum formulation that can achieve high performance efficiency and interpatient therapeutic variability (i.e. the same FPF at different shear stress/patient's maneuver). Furthermore, larger $k_{d}$ corresponds to a steeper rise in the variant region and FPF reaches maximum at lower shear stress. This is a desired feature because it reduces inter-patient variability when different inhalation maneuvers are implemented. However, formulations with larger $k_{d}$ often perform poorer than those with smaller $k_{d}$ at high shear when reaching $F P F_{\max }$. This phenomenon will be addressed in Chapter 5. It should be noted that shear stress is a characteristic and fundamental parameter of interfaces. It was of interest to directly relate macroscopic phenomena of an aerosol performance with the interfacial properties of shear stress. (see Chapter 5). The shear stress-dependent region can provide a unique opportunity to study the mechanism of powder deaggregation. According to Kendall's theory,[31] the separation of drug particles from the surfaces of carrier particles occurs when the energy consumption provided by shear force is proportional to the formation of new surfaces. In other word, the magnitude of shear stress (i.e. a linear motion that is exerted in two dimensions) must be sufficient to disrupt the favorable net interfacial forces at the drug/carrier solid-solid interface. Quantitatively, this is reflected in the unique PADE that directly related the performance result with shear stress. A hypothetically ideal deaggregation constant $\left(k_{d}=1\right)$ could be introduced to the 
PADE that accounted for the situation where aggregation and deaggregation are in equilibrium.[27] When $k_{d}=1$, the PADE (Langmuir equivalent) equation reduced to Eq. 4-3, when the performance efficiency (FPF) is dependent only on the applied shear stress:

$$
\tau_{s}=\frac{F P F}{F P F_{\max }-F P F}
$$

By using formulation with $k_{d}$ close to 1 , the magnitude of shear stress required to reach a certain FPF could be estimated. This feature could be used for device design when the turbulent shear stress is the principal mechanism of deaggregation.

\subsection{Summary}

Although lactose monohydrate dominates the carriers in the current marketed dry powder aerosol formulation, the formulation performance efficiency has been sub-optimal. Two trends are often used for carrier modifications and performance optimization. They include searching non-lactose sugars or sugar alcohols as alternative carriers, or surface modification of existing lactose monohydrate carriers. This chapter represents the first trend, while the second trend will be explored in Chapter 6.

The studies in this chapter were focused on: 1) rational evaluation of non-lactose based sugar (Treh) and sugar alcohol (Mann) and their potential as carriers for dry powder aerosol formulation; 2) performance studies of formulations using these alternative carriers (4 drugs $\times 2$ carriers); 3) SETs were used and the predictive behavior and robustness of PADE method was challenged and result showed that PADE non-linear 
regression and linear regression gave good to excellent correlation indicated by $R^{2}$ and adjusted $R^{2}$. Some mechanistic points of view regarding the physical meaning of the PADE interpretation were being formed. In the PADE non-linear regression analysis, the invariable (shear stress independent) region at high shear had important predictive implications for rationally designing or predicting formulation with high performance efficiency (represented by high $F P F_{\max }$ ). Furthermore, the variable (shear stress dependent) region at low shear reflected in the specific interfacial and physicochemical properties of drugs and carriers. Given enough data points across the shear stress at this region, it has the potential to quantitatively determine the heterogeneity at the solid-solid interface. This point will be addressed in Chapter 5. 
Figure 4-1. Schematic Representation of Potential Sugars and Sugar Alcohols Explored for Dry Powder Formulation Carriers. (A) D-glucose; (B) D-trehalose; (C) D-maltose; (D) D-raffinose; (E) erythritol; (F) D-xylitol; (G) D-mannitol; (H) D-sorbitol; (I) D-maltitol.

(A)

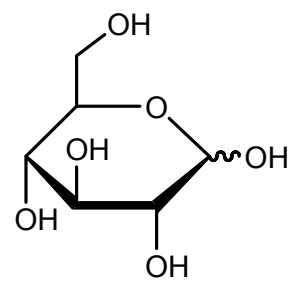

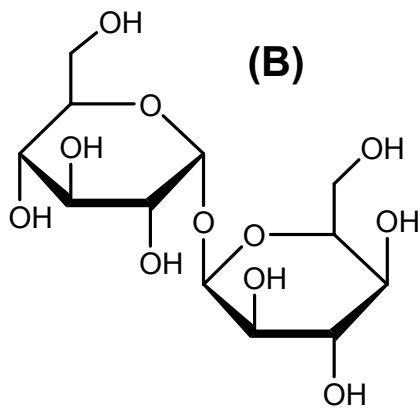

(C)
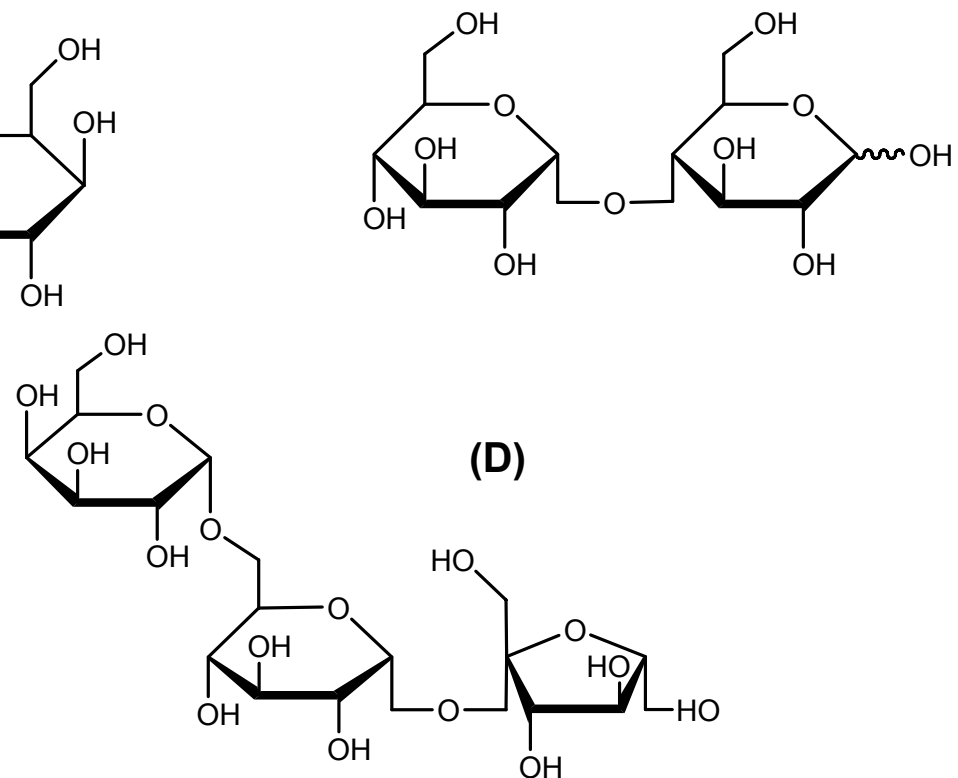

(E)<smiles>OC[C@H](O)[C@H](O)CO</smiles>

(F)<smiles>OC[C@H](O)[C@H](O)[C@H](O)CO</smiles>

(G)

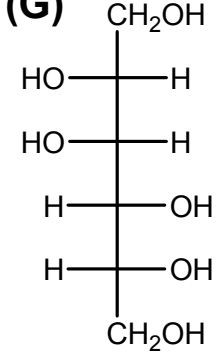

(H) $\mathrm{CH}_{2} \mathrm{OH}$<smiles>CC(O)C(O)C(O)C(O)CO</smiles>

(I)

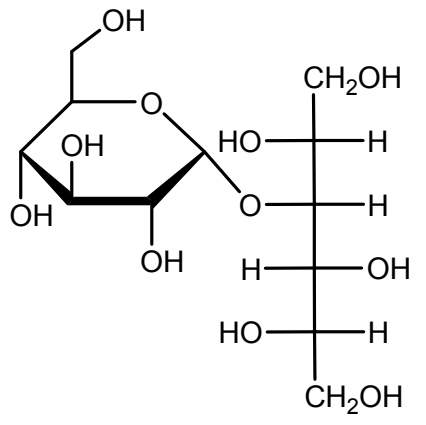


Figure 4-2. Representative Scanning Electron Micrographs (SEM) of Sugars $(1,500 \times$ magnification) and Interactive Physical Mixtures $(600 \times)$ of Micronized Pulmonary Drug/Sugar Carrier Systems in the Solid-state: (A) D-Trehalose dihydrate; (B) D-Mannitol; (C) Albuterol sulfate/D-Trehalose dihydrate; (D) Albuterol sulfate/D-Mannitol;

(A)

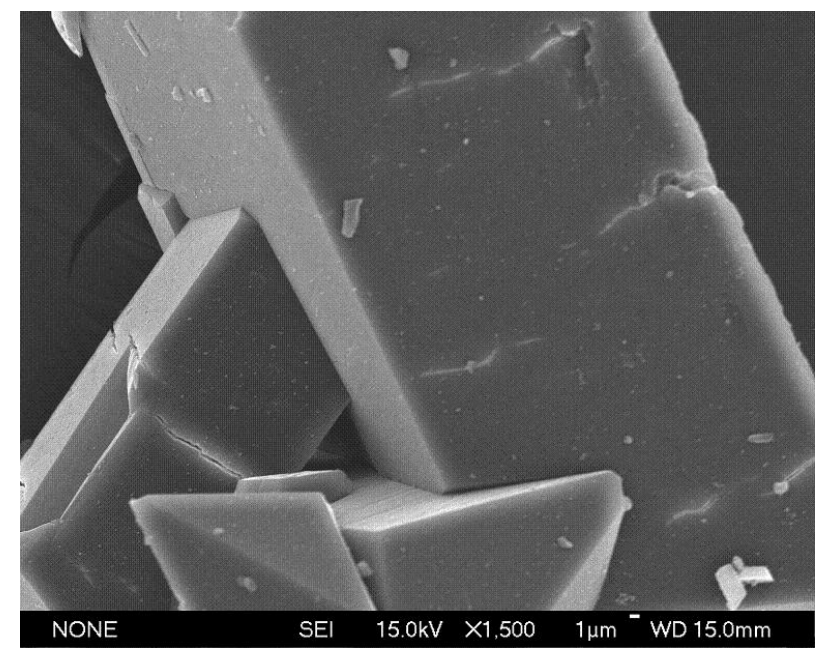

(C)

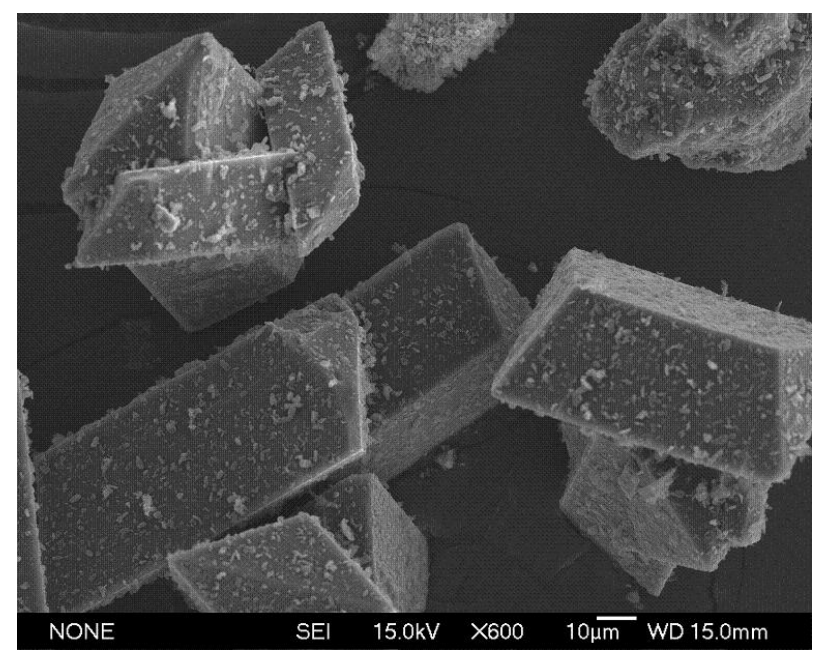

(B)

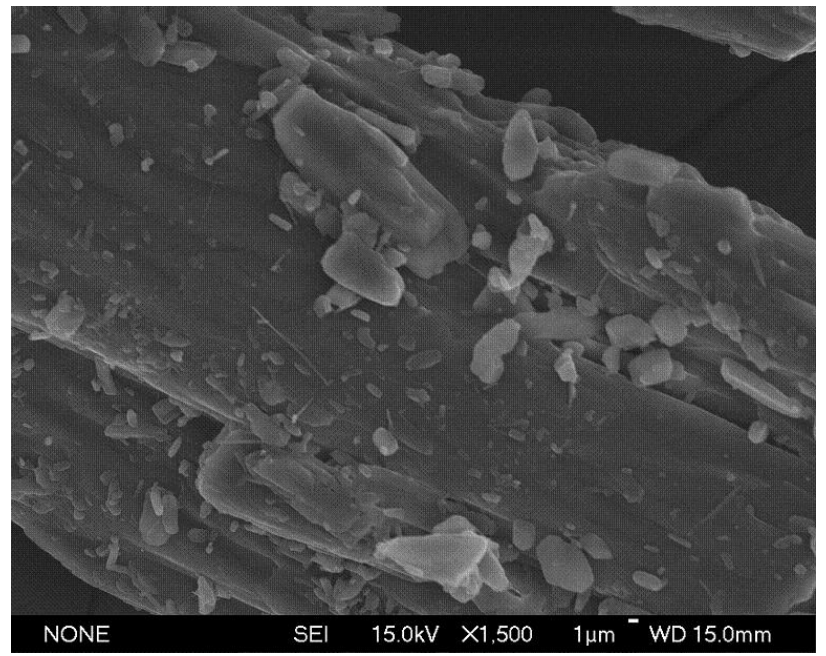

(D)

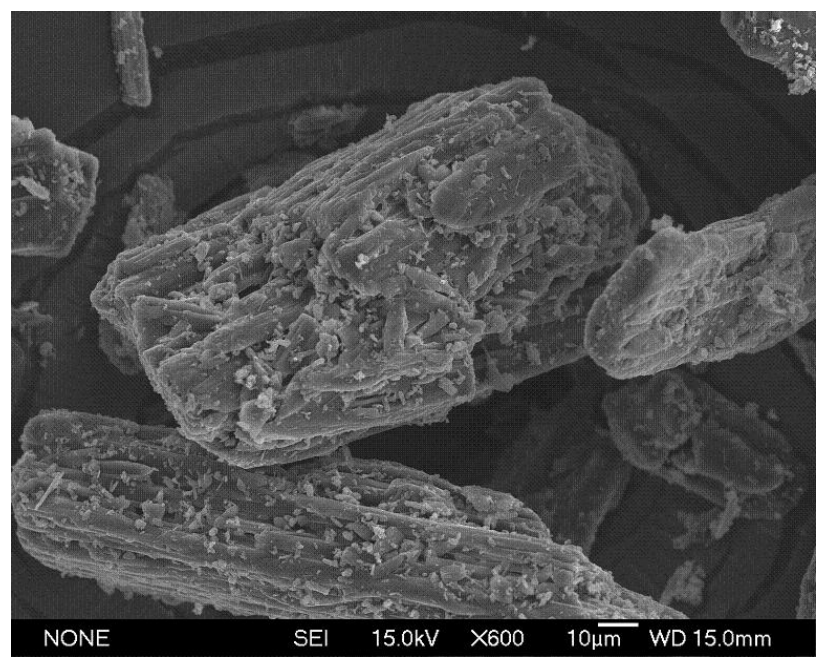


Figure 4-3. Representative Scanning Electron Micrographs (SEM) of Interactive Physical Mixtures (600×) of Micronized Pulmonary Drug/Sugar Carrier Systems in the Solid-state (cont'd): (A) disodium cromoglycate/D-trehalose dihydrate; (B) disodium cromoglycate/D-mannitol; (C) ipratropium bromide monohydrate/D-trehalose dihydrate; and (D) ipratropium bromide monohydrate /D-Mannitol.

(A)

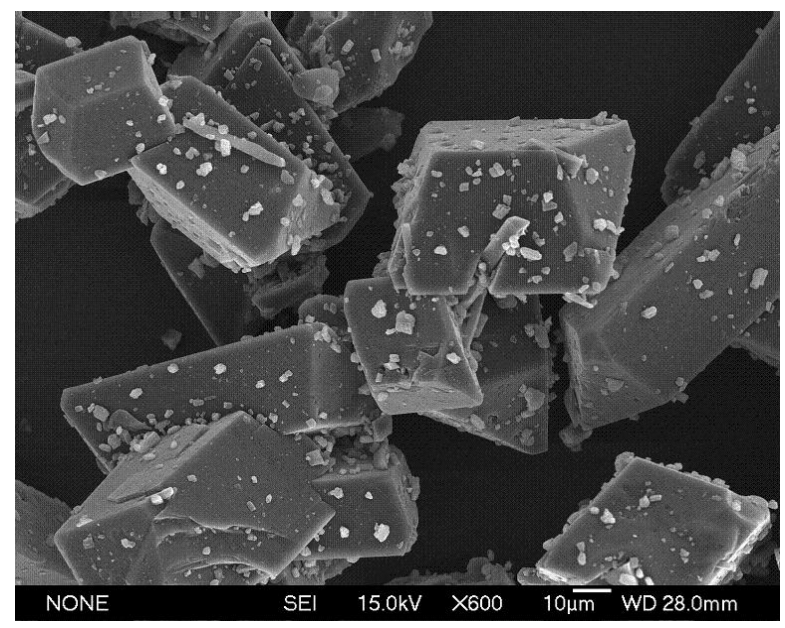

(C)

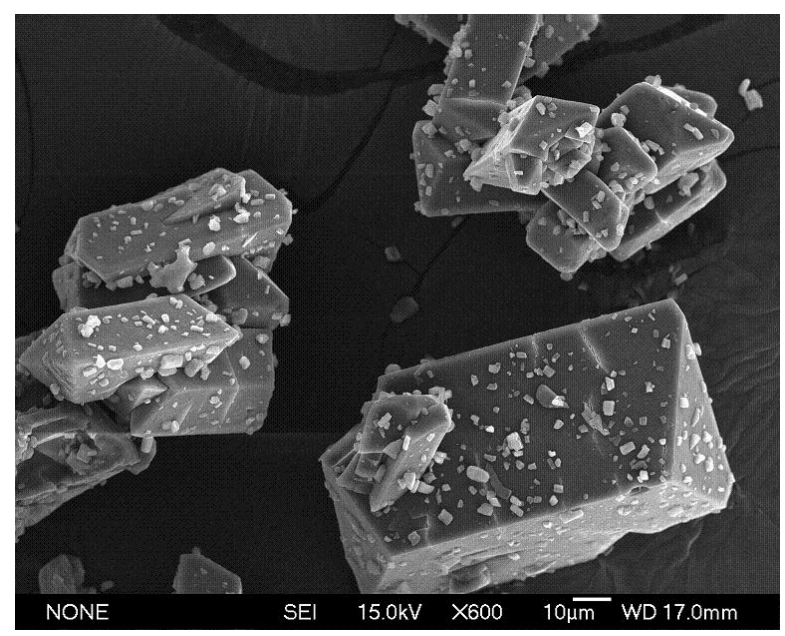

(B)

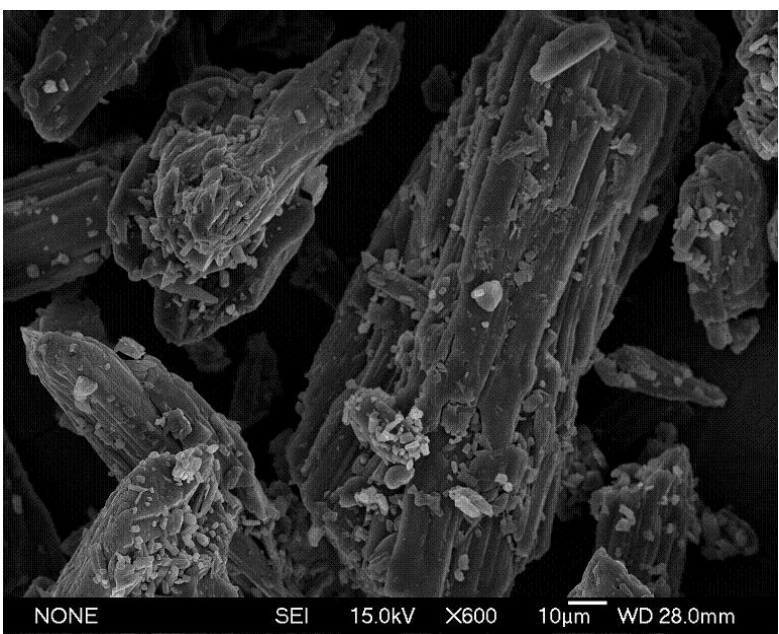

(D)

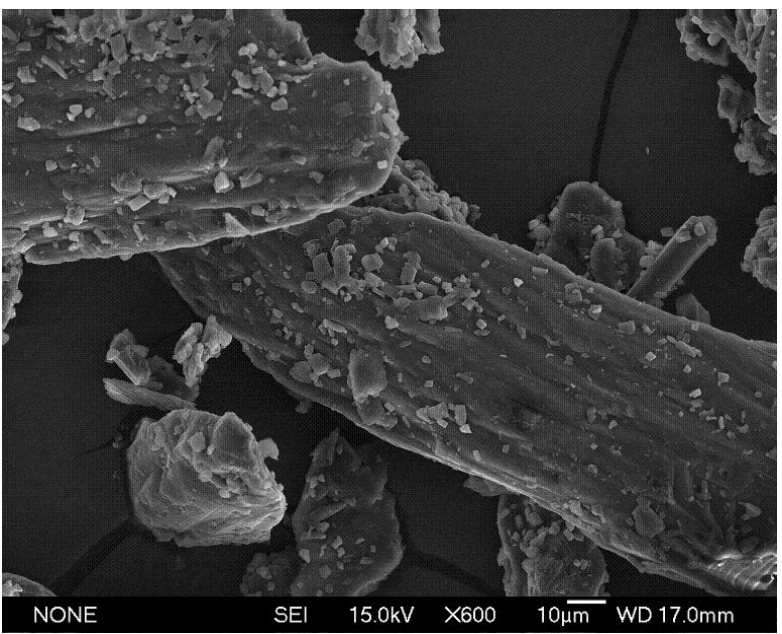


Figure 4-4. Representative Differential Scanning Calorimetry (DSC) Thermograms at a Heating Rate of $5{ }^{\circ} \mathrm{C} / \mathrm{min}$ for Carriers: D-mannitol (Mann) and D-trehalose dihydrate (Treh) in solid state: (A) Mann; (B) Treh (The $1^{\text {st }}$ scan: $5{ }^{\circ} \mathrm{C} / \mathrm{min}$ up to $220{ }^{\circ} \mathrm{C}$; the $2^{\text {nd }}$ scan: $20{ }^{\circ} \mathrm{C} / \mathrm{min}$ ).
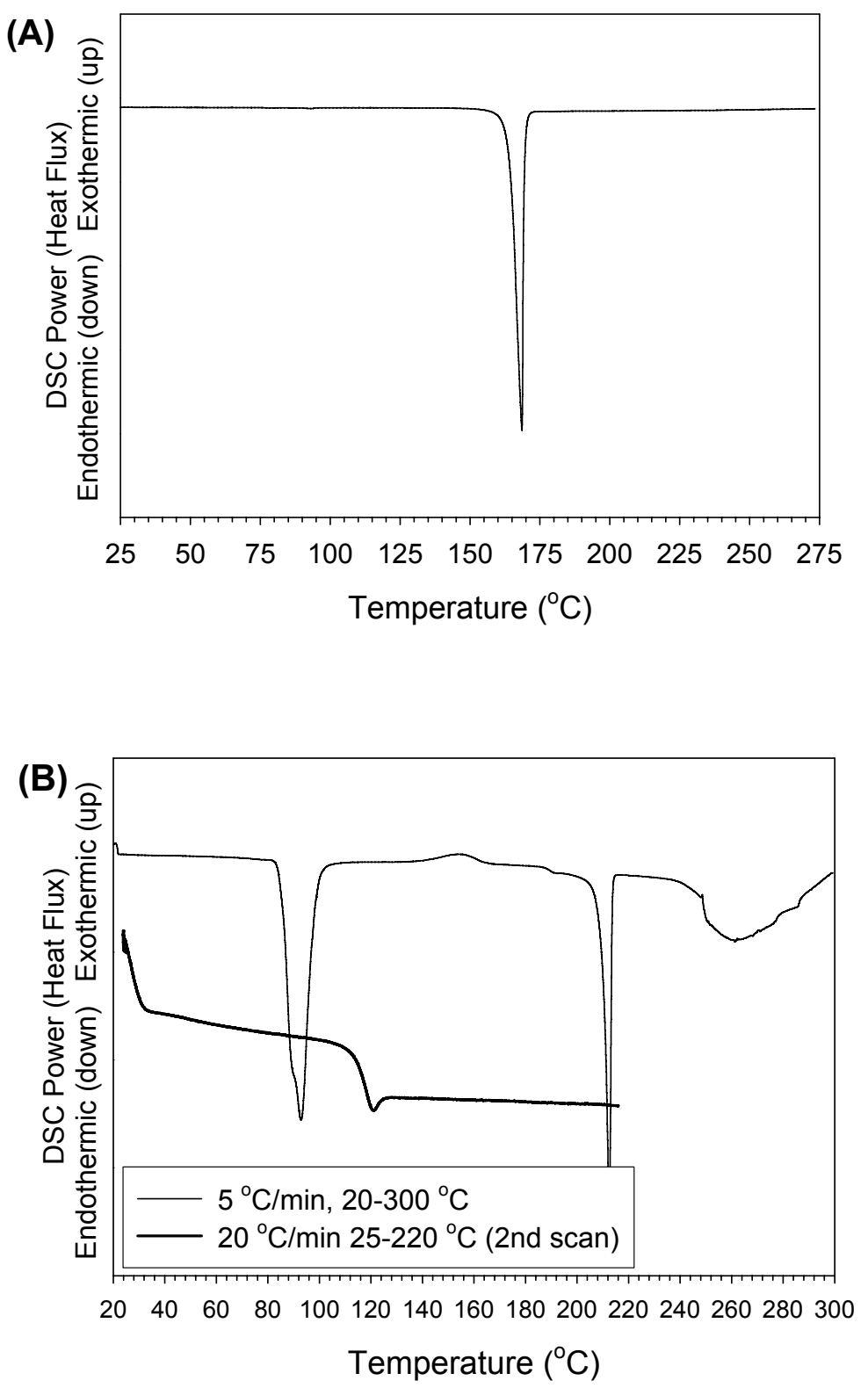
Figure 4-5. Representative DSC Thermograms at a Heating Rate of $5{ }^{\circ} \mathrm{C} / \mathrm{min}$ for Interactive Physical Mixtures. Drugs: albuterol sulfate (AS), disodium cromoglycate (DSCG), ipratropium bromide monohydrate (IPB), and fluticasone propionate (FP); Carriers: D-mannitol (Mann) and D-trehalose dihydrate (Treh) (sieved fraction: 45-75 $\mu \mathrm{m}$ ): (A). AS/Mann; (B). AS/Treh; (C). DSCG/Mann; (D). DSCG/Treh; (E). IPB/Mann; (F). IPB/Treh; (G). FP/Mann; and (H) FP/Treh.
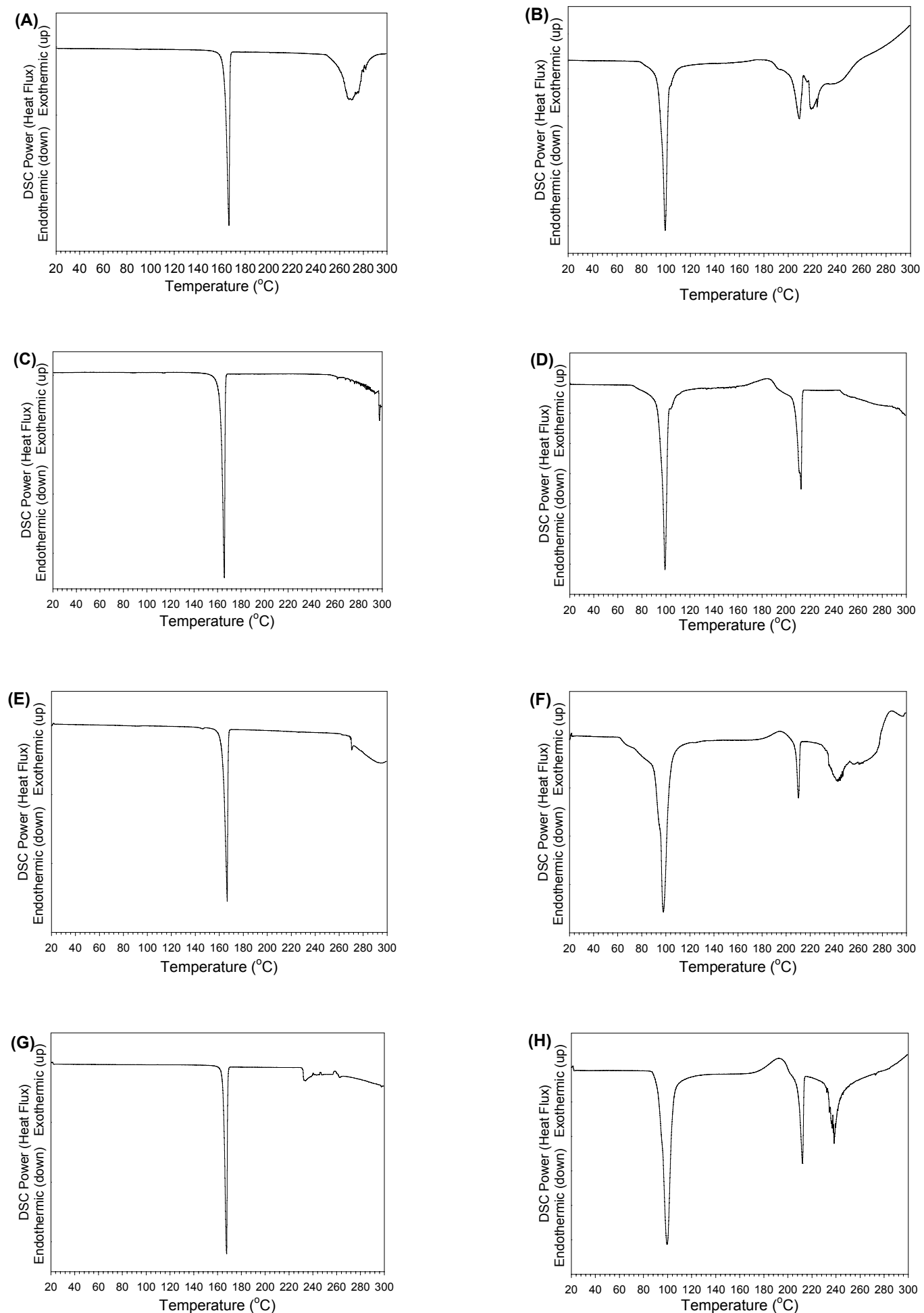
Figure 4-6. X-ray Powder (slit-detector) Height Intensity Diffractograms* of Alternative Sugar carriers (sieved fraction: $45-75 \mu \mathrm{m}$ ) at a scanning rate of $2 \theta$ degrees/minute: (A) D-mannitol; (B) D-trehalose dihydrate.

(A)

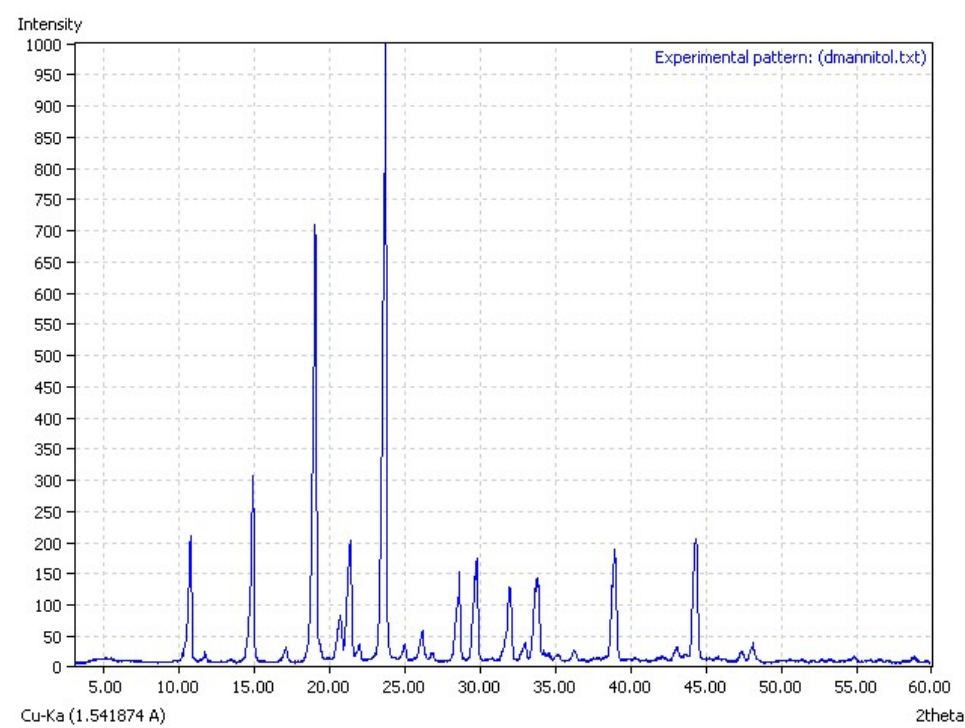

(B)

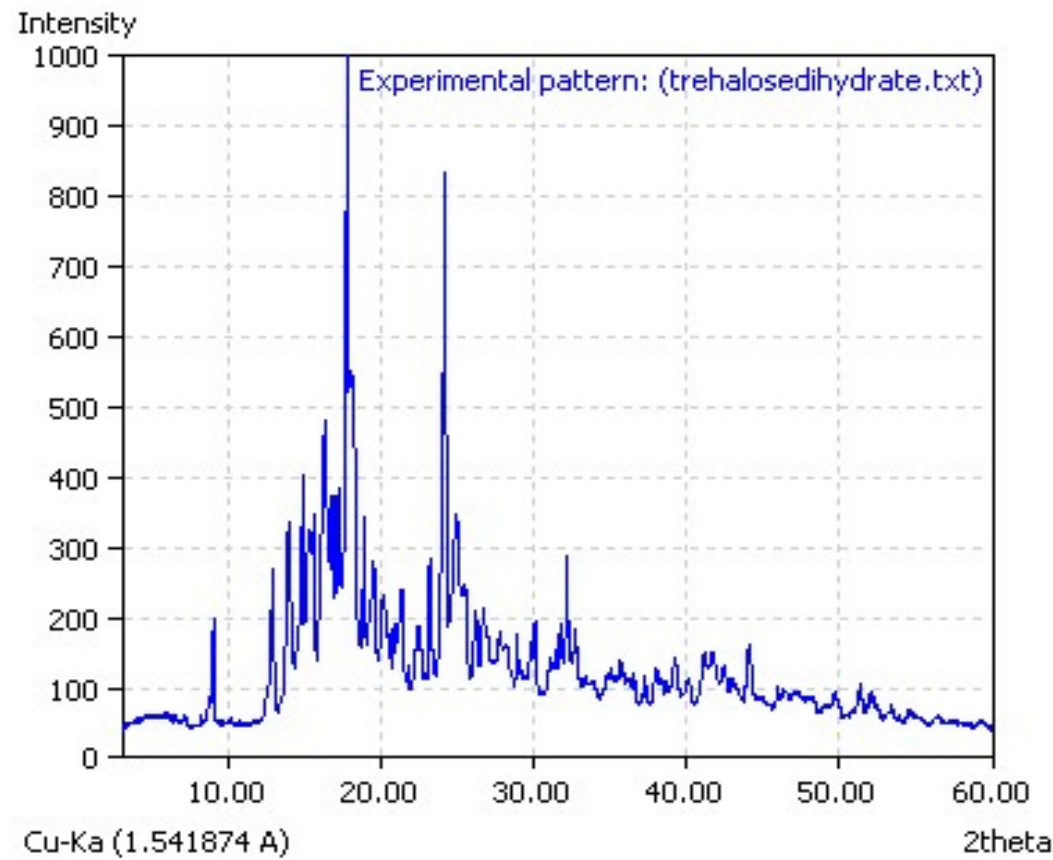

* Raw data: courtesy of Dr. Heidi Mansour. The data were re-plotted using Match ${ }^{\circledR}$ software, (Crystal Impact, Inc.) 
Figure 4-7. PADE Non-linear Regression Applied to TSLI Aerosolization $\left(\mathrm{FPF}_{\mathrm{TD}<6.4}\right.$ : mean $\pm \mathrm{SD}, n=3$ ) of dry powders for various interactive physical mixtures of micronized pulmonary drugs with trehalose dihydrate and D-mannitol sugar carrier systems: (A) albuterol sulfate; (B) ipratropium bromide monohydrate; (C) disodium cromoglycate; and (D) fluticasone propionate.
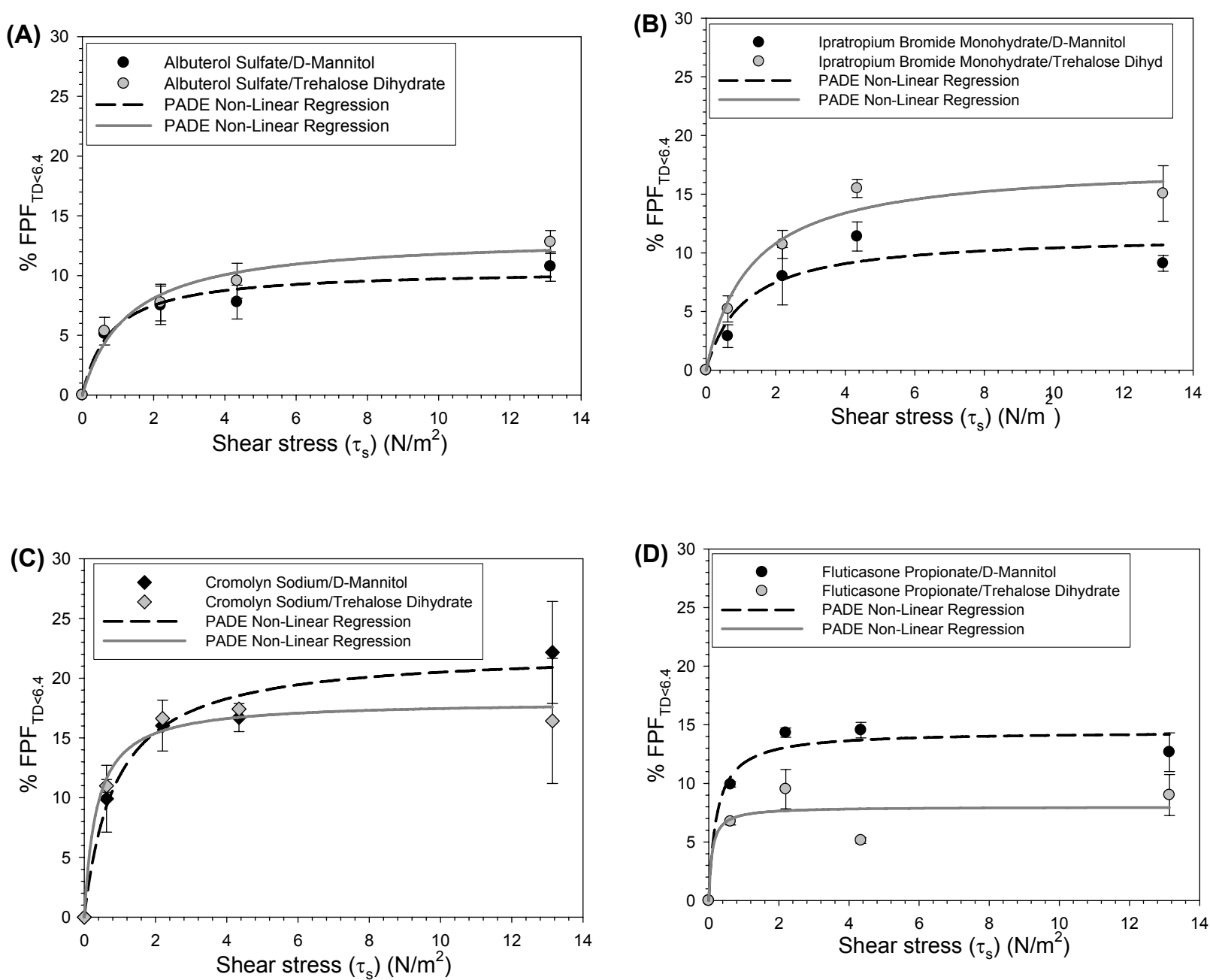
Figure 4-8. PADE Linear Regression Applied to TSLI Aerosolization $\mathrm{FPF}_{\mathrm{TD}<6.4}$ : mean value, $n=3$ ) of dry powders for various interactive physical mixtures of micronized pulmonary drugs with trehalose dihydrate and D-mannitol sugar carrier systems: (A) albuterol sulfate; (B) ipratropium bromide monohydrate; (C) disodium cromoglycate; and (D) fluticasone propionate. The $R^{2}$ of the linear regression is listed below:

(A)

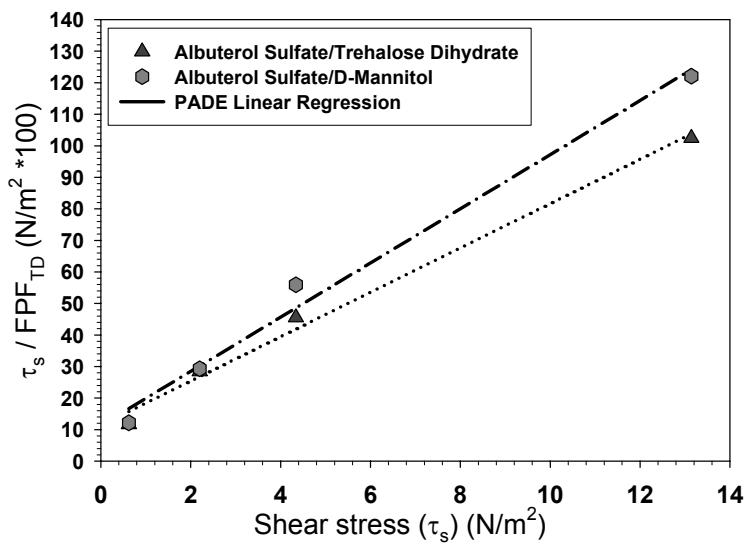

(C)

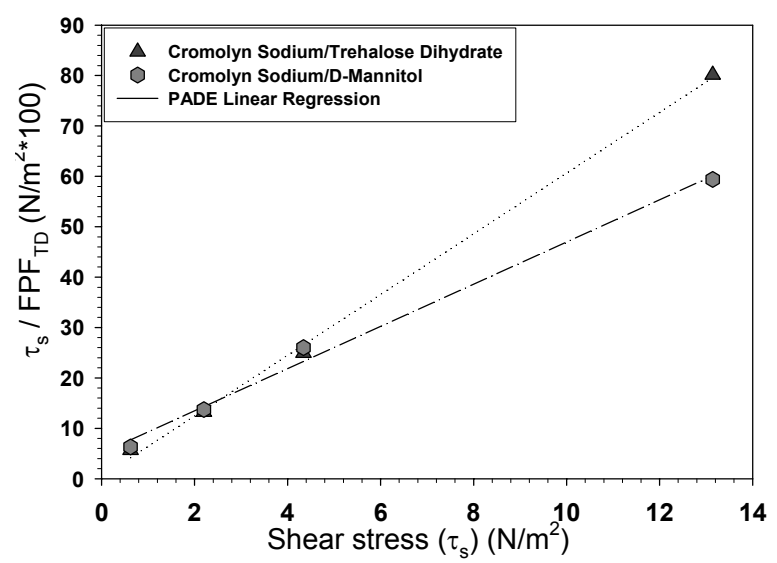

(B)

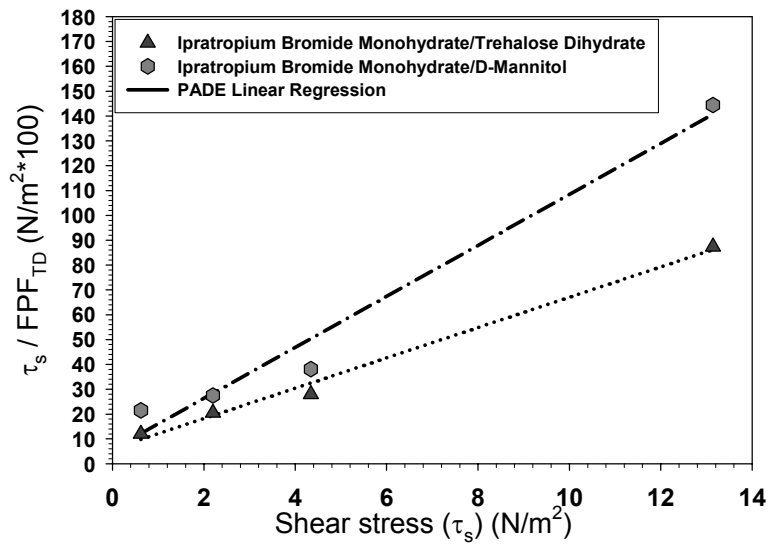

(D)

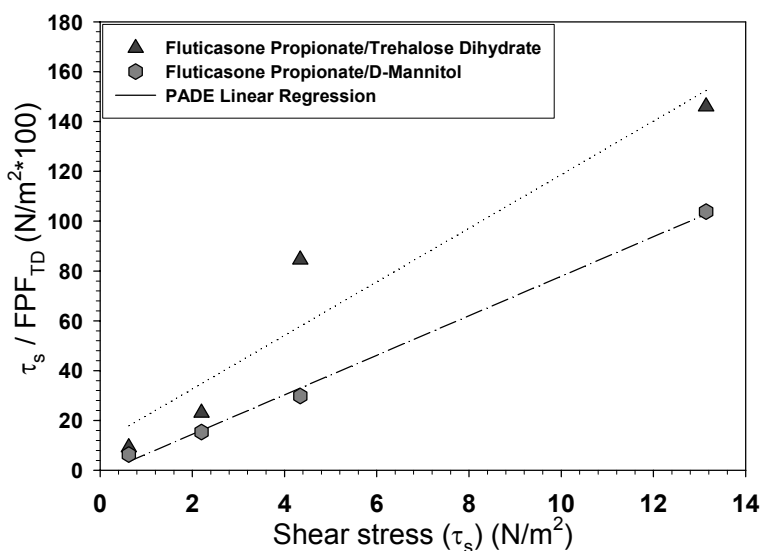

AS/Treh: $\mathrm{R}^{2}=0.9918$;

DSCG/Treh: $\mathrm{R}^{2}=0.9968$;

IPB/Treh: $\mathrm{R}^{2}=0.9909$;

FP/Treh: $\mathrm{R}^{2}=0.9274$
AS/Mann: $\mathrm{R}^{2}=0.9872$;

DSCG/Mann: $\mathrm{R}^{2}=0.9955$;

AS/Mann: $\mathrm{R}^{2}=0.9710$;

FP/Mann: $\mathrm{R}^{2}=0.9964$. 
Figure 4-9. The Relationship Between SET Shear Stress $\left(\tau_{s}\right)$ and TSLI Formulation Performance $(E D)$. Various interactive physical mixtures of micronized pulmonary drugs with trehalose dihydrate and D-mannitol sugar carrier systems: (A) albuterol sulfate (AS); (B) ipratropium bromide monohydrate (IPB); (C) disodium cromoglycate (DSCG); and (D) fluticasone propionate (FP).
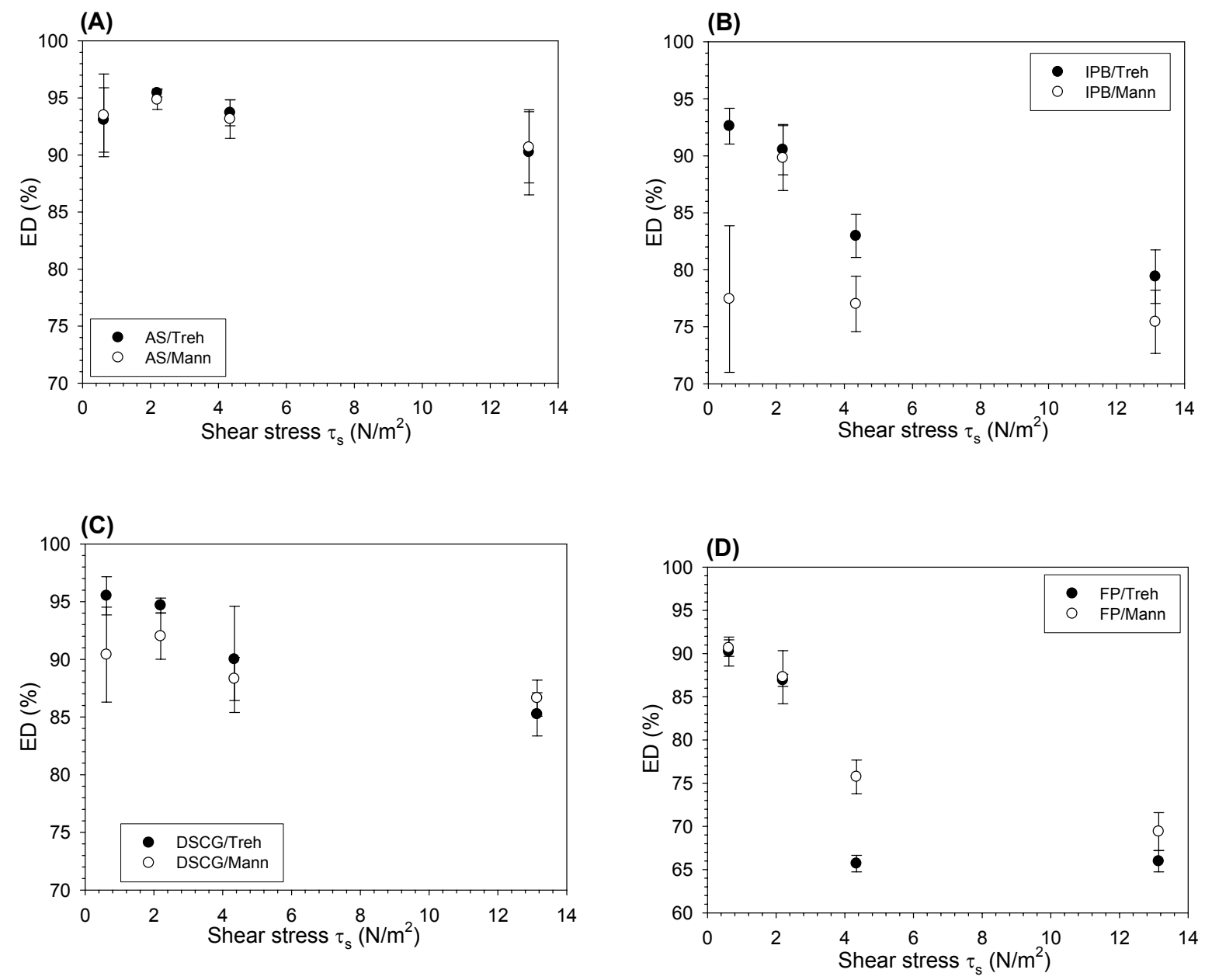
Table 4-1. Particle Volume Size Distribution of D-Mannitol (Mann) and D-Trehalose Dihydrate (Treh) (sieved fraction 45-75 $\mu \mathrm{m}$ ).

\begin{tabular}{|c|c|c|c|c|}
\hline & Span & $\mathbf{D}_{\mathbf{1 0}}(\boldsymbol{\mu m})$ & $\mathbf{D}_{\mathbf{5 0}}(\mu \mathbf{m})$ & $\mathbf{D}_{\mathbf{9 0}}(\boldsymbol{\mu m})$ \\
\hline Mann & $1.19 \pm 0.02$ & $13.49 \pm 0.24$ & $52.95 \pm 1.69$ & $76.98 \pm 0.18$ \\
\hline Treh & $0.84 \pm 0.04$ & $28.86 \pm 1.35$ & $53.49 \pm 0.79$ & $73.83 \pm 0.12$ \\
\hline
\end{tabular}


Table 4-2. TSLI Performance Data Analysis. The relationship between SET shear stress $\left(\tau_{s}\right)$ and $F P F_{T D<6.4} / F P F_{E D<6.4}$ for Treh-based and Mann-based dry powder formulations using non-linear regression analysis. $(n=3)$.

$$
\left.\frac{F P F}{F P F_{\max }}=\frac{k_{d} \tau_{s}}{1+k_{d} \tau_{s}} \quad \text { (Eq. } 4-1\right)
$$

\begin{tabular}{|c|c|c|c|c|c|c|}
\hline & Carrier & Drug & $\mathbf{F P F}_{\max }$ & $\mathbf{k}_{\mathbf{d}}$ & $\mathbf{R}^{2}$ & $\operatorname{Adj} R^{2}$ \\
\hline \multirow{8}{*}{$\mathrm{FPF}_{\mathrm{TD}}$} & \multirow{4}{*}{ Treh } & AS & 13.31 & 0.78 & 0.9728 & 0.9637 \\
\hline & & DSCG & 18.04 & 2.89 & 0.9845 & 0.9793 \\
\hline & & IPB & 17.62 & 0.79 & 0.9711 & 0.9614 \\
\hline & & $\mathrm{FP}$ & 8.00 & 10.88 & 0.8019 & 0.7359 \\
\hline & \multirow{4}{*}{ Mann } & AS & 10.50 & 1.24 & 0.9646 & 0.9528 \\
\hline & & DSCG & 22.29 & 1.14 & 0.9812 & 0.975 \\
\hline & & IPB & 11.56 & 0.91 & 0.8985 & 0.8647 \\
\hline & & $\mathrm{FP}$ & 14.44 & 4.31 & 0.9654 & 0.9538 \\
\hline & & & & & & \\
\hline \multirow{8}{*}{$\mathrm{FPF}_{\mathrm{ED}}$} & \multirow{4}{*}{ Treh } & AS & 14.98 & 0.66 & 0.9651 & 0.9535 \\
\hline & & DSCG & 20.67 & 2.18 & 0.9943 & 0.9924 \\
\hline & & IPB & 22.54 & 0.61 & 0.9700 & 0.9600 \\
\hline & & $\mathrm{FP}$ & 12.00 & 2.39 & 0.8612 & 0.8149 \\
\hline & \multirow{4}{*}{ Mann } & AS & 11.57 & 1.13 & 0.9577 & 0.9437 \\
\hline & & DSCG & 25.93 & 0.97 & 0.9792 & 0.9722 \\
\hline & & IPB & 15.20 & 0.75 & 0.898 & 0.864 \\
\hline & & FP & 19.94 & 2.08 & 0.9886 & 0.9849 \\
\hline
\end{tabular}




\section{References}

1. H. Steckel and N. Bolzen, Int J Pharm, 270, 297-306 (2004).

2. X.M. Zeng, H.B. MacRitchie, C. Marriott and G.P. Martin, Int J Pharm, 333, 45-55 (2007).

3. M.A. Braun, R. Oschmann and P.C. Schmidt, Int J Pharm, 135, 53-62 (1996).

4. J.C. Hooton, M.D. Jones and R. Price, J Pharm Sci, 95, 1288-1297 (2006).

5. J.C. Hooton, M.D. Jones, H. Harris, J. Shur and R. Price, Drug Dev Ind Pharm, 34, 974-983 (2008).

6. D. Cline and R. Dalby, Pharm Res, 19, 1274-1277 (2002).

7. D. Traini, P.M. Young, M. Jones, S. Edge and R. Price, Eur J Pharm Sci, 27, 243-251 (2006).

8. K. Endo, S. Amikawa, A. Matsumoto, N. Sahashi and S. Onoue, Int J Pharm, 290, 63-71 (2005).

9. S.K. Tee, C. Marriott, X.M. Zeng and G.P. Martin, Int J Pharm, 208, 111-123 (2000).

10. G. Saint-Lorant, P. Leterme, A. Gayot and M.P. Flament, Int J Pharm, 334, 85-91 (2007).

11. P. Harjunen, T. Lankinen, H. Salonen, V.P. Lehto and K. Jarvinen, Int J Pharm, 263, 151-163 (2003).

12. J. Patton and R.M. Platz, Adv Drug Deliv Rev, 8, 179-196 (1992).

13. N.W. Solomons, Rev Invest Clin, 48 Suppl, 1-13 (1996).

14. J. Kawanabe, M. Hirasawa, T. Takeuchi, T. Oda and T. Ikeda, Caries Res, 26, 358-62 (1992).

15. B.C. Hancock and S.L. Shamblin, Pharm Sci Technol Today, 1, 345-351 (1998).

16. K. Michio, S. Ikuo, O. Kazuyuki, T. Kano and M. Sae, J Appl Glycosci, 51, 63-70 (2004).

17. A. Patist and H. Zoerb, Colloids and surfaces B: biointerfaces, 40, 107-113 (2005). 
18. V.T. Huang, F.A. Panda, D.R. Rosenwald and K. Chida, Food product with enhanced crispiness U.S. 6,824,799 B1. (2004).

19. E. Schwarz, in: Ullman's Encyclopedia of Industrial Chemistry, B. Elvers, S. Hawkins, and W. Russey (Eds.), Vol. A25, pp. VCH, Weinheim (1994).

20. X.M. Zeng, G.P. Martin and C. Marriott, Particulate Interactions in Dry Powder Formulations for Inhalation. Taylor \& Francis, New York (2001).

21. A.J. Hickey, H.M. Mansour, M.J. Telko, Z. Xu, H.D. Smyth, T. Mulder, R. McLean, J. Langridge and D. Papadopoulos, J Pharm Sci, 96, 1282-1301 (2007).

22. A.J. Hickey, H.M. Mansour, M.J. Telko, Z. Xu, H.D. Smyth, T. Mulder, R. McLean, J. Langridge and D. Papadopoulos, J Pharm Sci, 96, 1302-1319 (2007).

23. A.J. Hickey and H.M. Mansour, in: Modified-release Drug Delivery Technology, M.J. Rathbone, et al, Vol. 2, pp. 573-602. Informa Healthcare, New York (2008).

24. A.J. Hickey and H.M. Mansour, in: Modern Pharmaceutics, A.T. Florence and J. Siepmann, Vol. 2, pp. 191-219. Taylor \& Francis, New York (2009).

25. Z. Xu, H.M. Mansour, T. Mulder, R. McLean, J. Langridge and A.J. Hickey, $J$ Pharm Sci, (2010 in press).

26. Z. Xu, H.M. Mansour, T. Mulder, R. McLean, J. Langridge and A.J. Hickey, $J$ Pharm Sci, (2010 in press).

27. H.M. Mansour, Z. Xu and A.J. Hickey, J Pharm Sci, (2010 in press).

28. L.R. Chen, V.G. Young, Jr., D. Lechuga-Ballesteros and D.J. Grant, J Pharm Sci, 88, 1191-200 (1999).

29. J.S. Cox, G.D. Woodard and W.C. McCrone, J Pharm Sci, 60, 1458-65 (1971).

30. M.D. Jones, J.C. Hooton, M.L. Dawson, A.R. Ferrie and R. Price, Int J Pharm, 313, 87-98 (2006).

31. J.R. Coury and M.L. Aguiar, Powder Tech., 85, 37-43 (1995). 


\section{CHAPTER 5 THEORETICAL DATA INTERPRETATION}

\subsection{Introduction}

Despite several decades of very active research in the field of dry powders prepared for inhalation, they remain complex and poorly understood systems. A direct correlation of particulate interaction with the aerosol performance is difficult because of the heterogeneous nature of both drug and carrier surfaces, and assumptions with respect to force measurement. The prediction and reproducibility of performance of pharmaceutical dry powder aerosols remains empirical. Under these circumstances, it is desirable to reassess the relationships between the fundamental intermolecular and surface forces, the deaggregation forces, and the aerodynamic behavior of the particulate systems.

In Chapter 1 (Section 1.4), the fundamental forces for particle adhesion/cohesion and deaggregation in pharmaceutical dry powder aerosol formulations were discussed. Current trends for performance prediction and optimization of dry powder aerosol formulation have been reviewed (see Section 1.5). The previous two sections are the foundation of experimental work on which the theory of powder aerosol deaggregation equations (PADE) is based. A great deal of experimental evidence shown in Chapter 3 and 4 indicated the robustness of the PADE in evaluating the aerosolization performance in the defined shear stress range. Based on the experimental data collected, for a total of twentyfour formulations (i.e. both the lactose monohydrate-based and two alternative sugar-based formulations, and four different drugs.), all resulted in excellent correlations when PADE was applied. 
In this chapter, the theoretical foundation for the PADE will be developed in four sections. (I) Description of PADE as an analogy to Langmuir adsorption isotherm; (II) "Experimental" justification of PADE based on performance of carrier-based dry powder formulation and statistical analysis. (III) Discussion and mechanistic interpretation of PADE for heterogenous particle deaggregation. (IV) Conclusion.

\subsection{PADE - An Analogy to Langmuir Adsorption Isotherm}

\subsubsection{Surface Adsorption Theory}

A potentially predictive and quantitative correlation of performance with aerosol formulation properties in response to a range of airflows has not previously been reported. To introduce a new method that describes the heterogeneous particle deaggregation across the entire shear stress range in detail, consideration of the fundamental theories applied for physical surface adsorption on solids that were developed in the late $19^{\text {th }}$ and $20^{\text {th }}$ century may be enlightening.

In 1916, Langmuir [1] stated "cohesion, adsorption and surface tension are all manifestations of forces similar in their nature to those acting between the atoms of solid bodies...". He presented a large amount of evidence in support of the nature of adsorption. Two years later, he proposed a monolayer adsorption theory of gas molecules onto a flat surface based on a kinetic principle that there is a continual process of adsorption of molecules onto the surface and a corresponding desorption (evaporation) of molecules, and at dynamic equilibrium the rate of adsorption is equal to the rate of desorption from the surface.[2] The forces acting between the adsorbate gas molecules and the solid adsorbent are mainly van der Waals forces, and this physical adsorption process is reversible (i.e. adsorption $\leftrightarrow$ desorption).[3] Relating the dynamic equilibrium with surface fractional 
coverage of gas molecule $(\theta)$ at constant temperature, he obtained the Langmuir adsorption isotherm (Eq. 5-1a or 5-1b):

$$
\begin{gathered}
\theta=\frac{k P}{1+k P} \quad \text { (Eq. 5-1a) } \\
\text { or } \quad \Gamma=\frac{\Gamma_{\max } k P}{1+k P} \quad \text { (Eq. 5-1b) }
\end{gathered}
$$

where $\Gamma$ is the amount of gas adsorbed $(\mathrm{g}) ; \Gamma_{\max }$ is the maximum adsorption to a monolayer surface coverage $(\mathrm{g}) ; P$ is gaseous partial pressure $\left(\mathrm{N} / \mathrm{m}^{2}\right)$; and $k$ is the Langmuir adsorption constant $\left(\mathrm{m}^{2} / \mathrm{N}\right)$, which is a measure of how strong an adsorbate molecule is attracted onto a surface. Later on, based on a large number of experimental data, the Langmuir theory was adapted to the solution adsorption isotherm (Eq. 5-2)

$$
\Gamma=\frac{\Gamma_{\max } k C}{1+k C} \quad(\text { Eq. 5-2) }
$$

where $\Gamma$ is the amount of solute adsorbed $(\mathrm{mg} / \mathrm{g}$ or $\mathrm{mmol} / \mathrm{g}) ; \Gamma_{\max }$ is the monolayer surface coverage $(\mathrm{mg} / \mathrm{g}$ or $\mathrm{mmol} / \mathrm{g}) ; k$ is still the Langmuir adsorption constant $(\mathrm{L} / \mathrm{mg}$ or $\mathrm{L} / \mathrm{mmol})$; and $P$ is replace by $C$, the final equilibrium concentration $(\mathrm{mg} / \mathrm{L}$ or $\mathrm{mmol} / \mathrm{L})$. Langmuir adsorption isotherm can also be derived from Gibbs thermodynamic approach when twodimensional solution expression is applied.[4]

Langmuir theory [4] assumes: 1) Adsorption molecules are adsorbed at definite, localized sites, each having an area $\sigma^{0}$. 2) Each site can accommodate only one molecule (monolayer coverage). 3) Adsorption energy is constant over all sites (homogeneity). 4) No lateral interaction exists between adsorbates. The Langmuir theory is generally accepted as the cornerstone of all theories of adsorption. It is the best known and most often used adsorption isotherms, accurately representing results. At low pressure or low concentration 
( $k P$ or $k C<<1$ ), the two-parameter Langmuir isotherm equation reduces to the Henry's law isotherm.

\subsubsection{Powder Aerosol Deaggregation Equation (PADE)}

Returning to the performance studies of dry powder aerosol formulations, the fundamental forces that contribute to the adhesion and deaggregation have been described in Chapter $\mathbf{1}$ (see Section 1.4). The forces acting at the particle interface are similar to those at the molecular level. So the Langmuir theory that describes molecular forces should, by analogy, be applicable to macroscopic particles, surfaces and even complex multicomponent systems.[5] The current techniques and methods for performance prediction have been reviewed (see Chapter 1, Section 1.5) and a promising trend for performance and airflow parameter correlation was observed. The pharmaceutical aerosol formulations involve hetereogeneous particles with different energy density regions. Similar to the heterogeneous adsorption phenomena, micronized drug particles prefer to adhere (or adsorb) onto sites having higher energy "active" sites. When these "active" sites are gradually occupied, the drug particles adhere onto progressively lower energy density sites, resulting in a decreased heat of adhesion (or from the guiding analogy, adsorption) with loading. When a certain shear stress is applied, those drug particles at lower energy density sites are preferentially deaggregated while those at higher energy density sites remain associated unless higher shear stress is applied. At a certain point, the shear stress is no longer able to remove more drug particles and reaches $F P F_{\max }$. At the fundamental level, we propose, that the models of molecular surface association described by adsorption expressions can be adapted to fit shear displacement observations. The algebraic equivalent of Langmuir adsorption isotherm is 
shown in Eq. 5-3, and is referred to as the Langmuir equivalent expression of powder aerosol deaggregation equation (PADE) (see below):

$$
F P F=\frac{F P F_{\max } k_{d} \tau_{s}}{1+k_{d} \tau_{s}}
$$

where $F P F$ is the fine particle fraction below a certain cutoff size (\%). It has been shown in previous chapters that higher $F P F$ represents greater drug deaggregation, and higher $F P F$ was obtained with increasing turbulence whereas turbulence is not the sole mechanism of deaggregation;[6] The parameter $F P F_{\max }$ is the maximum achievable fine particle fraction (\%). Further increasing of FPF with increasing shear stress is not possible without comminution of the primary particles. This represents the efficiency of formulation performance when sufficient shear stress is applied and can be used as a standard for formulation optimization. The parameter $k_{d}$ is the deaggregation constant $\left(\mathrm{m}^{2} / \mathrm{N}\right)$. This constant represents the ease of drug deaggregation from carrier particles or agglomerates; And $\tau_{s}$ is the viscous shear stress $\left(\mathrm{N} / \mathrm{m}^{2}\right)$ that is obtained for SETs using Kolmogorov turbulence theory (see Chapter 2, Section 2.2.4.1).[7] The shear stress considers both contributions of turbulent airflow at core and laminar shear force near the wall of SETs for drug deaggregation. The parameter, shear stress across the SETs has a contribution analogous to $P$ (Eq. 5-1b) or $C$ (Eq. 5-2) of Langmuir adsorption isotherm which can be derived from Gibbs thermodynamic approach using two-dimensional expression. (Both $\tau_{s}$ and $P$ have the same unit $\mathrm{N} / \mathrm{m}^{2}$, and this unit is also used for interfacial or surface tension). So shear stress should provide the best correlation with $F P F$ when compared with the other airflow parameters (Re, $\Delta P$, and power). 
The correlation of $F P F$ with $\tau_{s}$ using PADE (Langmuir equivalent expression) (Eq. 5-3) can be achieved by non-linear regression analysis (curve fitting) of each set of experimental data with any of various computer programs such as SigmaPlot, Origin, MatLab, Winnolin, and Igor Pro software. Here, SigmaPlot 9.0.1 software will be used for this analysis. The goodness of fit is evaluated from the error analyses including coefficient of determination $\left(R^{2}\right)$, adjusted $R^{2}$, the sum of squared errors $(S E)$, coefficient of variation (\%cv), and normality test.

The Langmuir adsorption isotherm is linearly transformable, and the linear models include Scatchard, Eadie-Hofstee, Lineweaver-Burk, and Langmuir linear equation (Figure 5-1). The choice of appropriate linear model depends on the $R^{2}$, the linearization without altering the error structure and normality assumption, and the meaningful physical interpretation of the selected model. The Scatchard model is the linear form of the binding equilibrium, which is derived from protein binding that follows the adsorptive phenomenon.[3] The Scatchard regression gives good representation of data fit for dilute solutions, but is biased in the high concentration range. The Lineweaver-Burk (doublereciprocal) and Eadie-Hofstee models are transformed from the Michaelis-Menten kinetics and often applied in the area of pharmacokinetics. They have bias to fit the data in the low concentration range. The Langmuir linear model was introduced by Langmuir himself to fit the data in surface adsorption phenomena. The Lineweaver-Burk model, which is sometimes referred to Langmuir linear model-II, is also applied as an alternative in surface adsorption fitting.[8] The Langmuir linear model is inherently biased toward fitting the data in the high concentration range. Furthermore, many surface adsorption models (which have been discussed below) such as Freundlich, Sips, and Fowler-Guggenheim expressions, are used as 
complementary or more rigorous treatment for the data fitting that consider the factors of surface heterogeneity and/or lateral interaction.[9]

For convenience and consistency with the previous chapters (Chapter $\mathbf{3}$ and $\mathbf{4}$ ) that described such a novel interpretation of drug aerosolization performance using Langmuir equivalent PADE and applied to shear displacement observations in pharmaceutical inhalation aerosols, additional fundamental surface adsorption theories such as Freundlich, Sips theories will be included to evaluate dry powder aerosol performance that are inherently heterogeneous. Likewise, these mathematical expression equivalents on the whole are referred to, for convenience, as PADE.

\section{3 "Experimental” Justification of PADE}

\subsubsection{The Different Linear Regression Models}

Using TSLI, a summary of 24 formulation performance data ( 4 drugs $\times 6$ excipients) at 4 different shear stress $\left(\tau_{s}\right)$ values has been examined (see Chapter 3 and 4). Four model drugs were chosen with distinct physicochemical properties and pharmacological mechanism of actions for the treatment of respiratory diseases (asthma and chronic obstructive pulmonary disease). Four lactose monohydrate carriers (sieved $\{\mathrm{SV}-\mathrm{A}, \mathrm{SV}-\mathrm{B}\}$, milled $\{\mathrm{ML}-\mathrm{A}$, and MLB $\}$ ) and two alternative carriers (D-Trehalose dihydrate $\{$ Treh $\}$ and D-Mannitol $\{$ Mann $\}$ )

were selected for the performance evaluation. The thorough physicochemical characterization of the drugs, carriers, and dry powder formulation using different techniques were described in previous chapters.[10-12] The particle size distribution of micronized drugs characterized by laser diffraction exhibited comparable volume median diameter $\left(\mathrm{D}_{50}\right)$ in the respirable range $(3.5 \sim 4.5 \mu \mathrm{m})$ with narrow unimodal distribution $(\operatorname{Span}<0.5)$. The 
values of $\mathrm{D}_{50}$ of carrier lactose monohydrate, mannitol, and trehalose dihydrate were comparable $(52 \sim 61 \mu \mathrm{m})$. However, the span of milled lactose monohydrate $(\sim 3.1)$ was much larger than sieved lactose monohydrate ( 1.2). The span of mannitol and trehalose dihydrate was $\sim 1.2$ and $\sim 0.8$, respectively. The performance data (exemplified as $F P F_{T D<6.4}$ which is defined as the percentage of mass of drug below $6.4 \mu \mathrm{m}$ cutoff from the total dose) using twin-stage liquid impinger (TSLI) was then correlated with shear stress $\tau_{s}$ of the SETs using PADE (Langmuir equivalent expression). The four different linear regression result including Langmuir, Scatchard, Eadie-Hofstee, and Lineweaver-Burk linear regressions is shown in Table 5-1. The differences between the linear forms of PADE significantly affected the regression result due to the influence of linearization process. The coefficients of determination $\left(R^{2}\right)$ ranges of these models are $0.9274-0.9999,0-0.9911,0-0.9911$, and 0.0007-0.9983, respectively. These data indicate that Langmuir, by virtue of variation in the linear regression analysis, is the most suitable and consistent model for all the data presented, followed by Lineweaver-Burk model. In contrast, Scatchard and Eadie-Hofstee linear regression did not result in good correlations for some of the IPB and FP formulations. The rank order based on the performance of $\left(F P F_{T D}\right)$ in Langmuir linear regression is shown below:

a. Rank order $\left(F P F_{\max }\right)$ of AS formulation: ML-A $>$ ML-B $>$ SV-B $>$ SV-A $>$ Treh $>$ Mann b. Rank order $\left(F P F_{\max }\right)$ of DSCG formulation: ML-B $>$ ML-A $>$ SV-B $>$ SV-A $>$ Mann $>$ Treh c. Rank order $\left(F P F_{\max }\right)$ of IPB formulation: ML-B $>$ ML-A $>$ SV-A $>$ SV-B $>$ Treh $>$ Mann d. Rank order $\left(F P F_{\max }\right)$ of FP formulation: $\quad$ ML-B $>$ ML-A $>$ SV-B $>$ SV-A $>$ Mann $>$ Treh It showed that the PADE (Langmuir equivalent expression) fitted the experimental data in all studied formulations. The parameter $F P F_{\max }$ is suitable for evaluating the efficiency of 
formulation performance and the result is consistent across different carrier-based dry powder formulation evaluated. It has also been observed that the SV lactose monohydrate formulations generally yield higher deaggregation constant $k_{d}$ than $\mathrm{ML}$ formulations, represented in PADE non-linear regression graphs are the sharp increase of the deaggregation curve at low shear stress of SV formulations.[10-13] Whereas SV formulation reaches saturation at much lower shear stress than ML formulation which implies that higher deaggregation formulations have lower deaggregation capacity. This phenomenon can be explained as more agglomeration of drug and fine particles in the ML formulation increases the capacity and result in higher $F P F_{\max }$.

\subsubsection{The Adaptation of Different PADE Models}

The adaptation of Langmuir adsorption isotherm to describe the deaggregation process is based on the assumption that there is neither surface heterogeneity nor lateral interaction, which is rarely true for relevant pharmaceutical aerosol formulations. Two specific aims were addressed in order to assess the mechanism of deaggregation accurately. The first aim was to adapt different surface adsorption models to fit the experimental dataset (PADE nonlinear regression). This is a method that is frequently applied for surface adsorption studied in many different systems, and a plethora theoretical and empirical adsorption isotherms have been developed or adapted to many complicated systems that consider surface heterogeneity, adsorbate mobility, lateral interaction, porous adsorbent, and multi-component adsorption. Due to the similarity (Table 5-2) of surface adsorption phenomena and the pharmaceutical aerosolization performance, and the good fit of aerosol performance data using algebraic equivalent Langmuir adsorption theory (Table 5-1), these adsorption isotherms or equations 
are arguably good candidate for adaptation to the performance studies. A comparison of PADE non-linear regression using algebraic equivalent Langmuir isotherm and Freundlich equation is shown in Table 5-3. The second aim was to introduce additional SETs to increase the number of data points for more accurate correlation. The airflow parameters of three additional SETs are listed in Table 5-4. Their dimension and geometry were designed and calculated based on the existing four SETs. (see Appendix 5-A). Two carrier-based formulations including DSCG/ML-B and AS/Mann were selected for this study. The selection of these two formulations is somewhat arbitrary, but the former represents the best performance formulation among all studied formulations and the latter represents an alternative sugar formulation.[10-13] The performance data are shown in Appendix 5-B). In this study, a comparison of three different algebraic equivalent surface adsorption expressions including Langmuir isotherm, Freundlich expression, and Sips expression were applied to fit the experiment dataset. The reasons for selecting these three algebraic equivalent surface adsorption models have their theoretical justification and will be discussed in the next section. The PADE non-linear curve fitting is illustrated in Figure 5-3 and the statistics in Table 5-5.

\subsection{Discussion}

5.4.1 Similarities \& Differences Between Surface Adsorption \& Aerosol Deaggregation

\section{(Table 5-2)}

The Langmuir adsorption isotherm, protein binding equilibria, Michaelis-Menten kinetics, and the novel PADE share an algebraically equivalent formula. However, they are inherently different expressions to account for different physicochemical phenomena. 
As is described above, Langmuir adsorption theory equates the rates of adsorption and desorption in term of fractional loading. It is time independent when a dynamic equilibrium is reached. Langmuir adsorption has fundamental theoretical basis. The isotherm equation reduces to the Henry law isotherm when the pressure is very low $(k P<<1)$. At high pressure, the amount adsorbed reaches the saturation capacity to form a monolayer surface coverage. When the Langmuir adsorption constant is larger, the surface is covered more with adsorbate molecules due to stronger affinity of adsorbate molecules towards the surface, so that the adsorption curve will have a more rapid increase with increasing pressure. Increase in the temperature or decrease the heat of adsorption results in reduced adsorption due to increased mobility of adsorbate molecules or reduced energy barrier for evaporation.[9] It should be noted that the physical adsorption is reversible whereas the chemisorption is irreversible and quite different, although they are expressed by the same formula. The heterogeneity of surface adsorption is caused by adsorbent geometric and energetic features, the adsorbate size, shape, and conformation, and an interaction between adsorbate-adsorbent. The treatment of heterogeneous adsorption is achieved by grouping the similar surface energy site in a patchwise or random manner using statistical thermodynamics and applies appropriate isotherm to each local adsorption (Figure 5-4).[9]

Similarly, the protein binding is also a time independent equilibrium process. The amount of adsorbed and monolayer surface coverage in Langmuir adsorption isotherm are now described as the percentage of drug bound in the total protein and the number of independent binding sites, respectively. The protein binding curve will have a more rapid increase when the association constant is large (affinity), whereas the number of binding sites determines the saturation capacity. The association constant is also temperature dependent. If 
there're multiple binding sites or binding sites are dependent one another (heterogeneity), the treatment is usually to group similar classes of binding sites, treat each class of protein binding separately and then add them up.[3]

The enzyme kinetics that governed by the Michaelis-Menten equation is algebraically equivalent to the Langmuir adsorption isotherm. But the physical meaning is the relationship between reaction rate with substrate concentration, which is quite different from either the surface adsorption or protein binding.

We can find some unifying concepts in dry powder aerosolization using SET, Langmuir adsorption, and protein binding. These physicochemical phenomena are governed by the same fundamental forces as we mentioned above. Besides the kinetic approach, the Langmuir adsorption equilibria can also be derived quite readily by statistical thermodynamic approach based on Gibbs law.[4, 14] The particle deaggregation process, despite its complexity, can possibly be explained by similar thermodynamic approach. The interfacial driving force in Langmuir adsorption isotherm is the gaseous partial pressure $P$ $\left(\mathrm{N} / \mathrm{m}^{2}\right)$ or solute concentration $C(\mathrm{mg} / \mathrm{g})$, while the aerosol entrainment and deaggregation requires the shear stress $\tau_{s}\left(\mathrm{~N} / \mathrm{m}^{2}\right)$ to overcome particulate adhesion. In other words, the separation occurs when the energy consumption provided by shear stress is proportional to the formation of new surfaces.[15] Corresponding to the surface coverage in adsorption, and number of independent binding sites in protein binding, the parameter in deaggregation expression is defined as, $F P F_{\max }$, the maximum achievable fine particle fraction. Furthermore, a striking analogy can be drawn to account for the heterogeneity and lateral interaction in surface adsorption, protein binding, and aerosol dry powder deaggregation. Among them, the heterogeneity in particle deaggregation is probably the most complicated because geometric, 
energetic characteristics of both drug particle and carrier particle, and the interfacial interaction of both particles contribute to the heterogeneity. The treatment of the surface heterogeneity can follow either the classifying of different binding site and add up for protein binding, or the patchwise treatment of each local adsorption (i.e. sites having the same surface adhesion energy are grouped together into one patch). The lateral interaction in surface adsorption is analogous to drug-drug cohesion or surface agglomeration.

Despite the unifying concepts between adsorption, protein binding, and aerosol deaggregation, there are fundamental differences between the powder deaggregation and the other two concepts. Both molecular adsorption/desorption (physical adsorption) and protein binding/disassociation are derived from time independent equilibria. They are reversible processes. However, the particulate adhesion/deaggregation is mainly a time dependent process. The particle entrainment and drug deaggregation, due to the particle size and system dimension, cannot be treated as a continuum for the continual process of drug particle adhesion and deaggregation from the carrier surface as equilibrium. There could be a small chance of drug/fine particles to adhere to other particles after deaggregation if there is sufficient settling time, but the chance of forming a dynamic equilibrium similar to interfacial adsorption or protein binding is remote. Furthermore, unlike surface adsorption and protein binding that have constant system volume and boundary condition, the particle deaggregation involves rapid system volume expansion and the corresponding net gain in entropy. It is not important that there is full coverage of the surface as is described for monolayer surface adsorption.

However, the analogy between these three processes will still hold, and can be used for explaining the experimental results in the dry powder aerosol performance. Similar to the 
Langmuir adsorption and protein binding, the deaggregation expression may serve as a link between macroscopic statistical thermodynamics and microscopic individual particle deaggregation.

\subsubsection{The Choice of Surface Adsorption Instead of Protein Binding}

The adaptation of Langmuir adsorption isotherm (both Langmuir adsorption and protein binding) for shear displacement (Eq. 5-3) has been tested by the aerosolization performance studies of a total of sixteen lactose monohydrate-based formulations, four D-trehalose dihydrate- and four D-mannitol-based formulations with four distinct drugs. In every case, the result has furnished striking confirmation of the correctness for such adaptation. Among the four studied linear regression analyses using PADE (Langmuir equivalent expression), Langmuir-type linear regression of performance and shear stress gave the most consistent and excellent correlation result (Table 5-1). Langmuir linear regression is widely used in gas or solution adsorption analyses. It indicates that this linear regression method tolerates the surface heterogeneity and lateral interaction, and still furnishes valuable deaggregation information from coefficient $\left(F P F_{\max }, k_{L}\right)$. However, linear regression such as Scatchard regression did not yield consistent correlation result. This is possibly because the process of heterogeneous particle deaggregation is quite complex and cannot be explained by a single class of binding sites. The treatment using multiple binding sites and add-up method is need. Due to the limitation of measuring each class of binding (adhesion) site, the adaptation using protein binding method may not be applicable. Furthermore, the scatchard plot requires the regression from both $\mathrm{X}$-axis $\left(F P F_{T D}\right)$ and $\mathrm{Y}$-axis $\left(F P F_{T D} / \tau_{s}\right)$, while the Langmuir linear regression regresses only on the $\mathrm{Y}$-axis $\left(\tau_{s} / F P F_{T D}\right)$. Besides, the surface adsorption studies 
has a long history starting from late nineteenth century and a plethora of theoretical and semi-empirical adsorption models have been developed based on different studied systems. [9, $16,17]$ Many of those models have rigorous theoretical basis or theoretical reasoning. With the foregoing background, the adaptation of surface adsorption models was selected as the model for PADE.

\subsubsection{PADE Considering Surface Heterogeneity}

The Langmuir adsorption expression is the cornerstone of almost all the surface adsorption isotherms and has been most widely used. Subsequently developed theoretical and semiempirical isotherms were based on experimental data and statistical thermodynamic derivation considering various influences such as affinity constant change (e.g. Volmer isotherm [9]), surface heterogeneity (e.g. Freundlich,[18] Sips,[19] Toth,[20] Unilan equation [9]), and lateral interaction (e.g. Fowler-Guggenheim equation [9]).

It is proposed that an expression that is algebraically equivalent to Langmuir adsorption theory is suitable to describe the deaggregation phenomenon. However, this treatment does not fully consider the surface heterogeneity between solid particles. The direct evidence of the surface heterogeneity is the convergent points among different regression lines/curves (Figure 5-2). In this case, several surface adsorptions equation equivalents that consider the surface heterogeneity were selected. The algebraic equivalent of these surface adsorptions could possibly be applied to the aerosolization performance as well. In this paper, the algebraic equivalents of Freundlich and Sips equation have been used as examples for alternative adaptation to the algebraic equivalent of Langmuir adsorption isotherm. 
The Freundlich equation is the earliest known adsorption expression that was developed empirically to account for non-ideal sorption of solute or gas molecules onto heterogeneous surfaces.[18] The equation takes the following form (Eq. 5-4a):

$$
\begin{aligned}
& C_{\mu}=K_{F} P^{1 / n} \\
& F P F=k_{F} \tau_{s}^{1 / n}
\end{aligned}
$$

where $C_{\mu}$ is the concentration of the adsorbed species, and $K_{F}$ and $n$ are two constants that relate to the heat of adsorption and heterogeneity factor. The corresponding algebraic equivalent of the Freundlich equation is shown in Eq. 5-4b. Because $n$ is an empirical parameter, it does not reveal the source of the heterogeneity during drug deaggregation. But similar to the $F P F_{\max }$ in Langmuir adsorption isotherm, it can be used to fit the dataset for macroscopic evaluation. The more heterogeneous the system for the drug deaggregation is, the larger $n$ will be. Unlike the Langmuir adsorption isotherm, the Freundlich equation does not have a fundamental thermodynamic basis since it does not have a proper Henry law behavior at low pressure, nor does it have a finite limit when pressure is sufficiently high (no saturation). Despite being an empirical equation, it has a theoretical basis for heterogeneous surface having patchwise local Langmuir adsorption mechanism and an exponential decay energy distribution.[9] Two assumptions are involved: 1) No interaction between patches and; 2) Adsorbate molecule occupies only one adsorption site.

A comparison of the PADE non-linear regression results using algebraic equivalent Langmuir and Freundlich equations is shown in Table 5-3. Interestingly, the non-linear curve fitting using Freundlich model also gave excellent correlation (adjusted $R^{2}: 0.7159-1.0000$ ) comparable to Langmuir model correlation (adjusted $R^{2}: 0.8655-0.9983$ ). The application of alternative surface adsorption isotherm for shear displacement observation adds strong 
evidence that the methodology of surface adsorption theory can be used to adapt the shear displacement of the aerosol formulation. It should be noted, however, that the parameter $n$ for empirical heterogeneity evaluation of our current data did not furnish useful result, although it is known that ML carriers are more heterogeneous than SV carriers from the physicochemical surface analyses. It indicates that the heterogeneity factor that influences drug deaggregation may not be a simple scenario. The extent of the heterogeneity may not only be determined by the geometric and energetic heterogeneity feature on both carrier and drug particles, but also account for the capacity for surface adhesion (e.g larger surface area), agglomerate formation or the ratio of cohesion-adhesion balance.

The initial attempt to increase the surface adsorption parameter from 2-parameter to 3-parameter PADE model was unsuccessful, though a higher coefficient of determination was obtained in many formulations. The results showed high dependency due to overparameterized model selection. In order to obtain more accurate data fitting, three additional SETs were prepared and two formulations were selected for further study. The correlation using three different algebraic equivalent surface adsorption models including Langmuir adsorption isotherm, Freundlich equation, and Sips equation (Eq. 5-5) were evaluated. (Figure 5-3 and Table 5-5). Sips equation (Eq. 5-5) is an extension of Freundlich equation having both a heterogeneity parameter $\left(n^{\prime}\right)$ and a finite saturation limit at high pressure or concentration. A slight increase in the $R^{2}$ was observed when a 3-parameter Sips adsorption model was applied compared with the other two adsorption models, but

$$
F P F=F P F_{\max }^{\prime} \frac{\left(k_{S} \tau_{s}\right)^{1 / n^{\prime}}}{1+\left(k_{S} \tau_{s}\right)^{1 / n^{\prime}}}
$$

the adjusted $R^{2}$ are comparable. It implied that the 3-parameter Sips model may not give more informative result than the Langmuir adsorption model. In order to obtain a more 
accurate PADE non-linear curve fitting, more data points are needed. In the assessments performed to support PADE theory, the algebraic equivalent of the Langmuir adsorption isotherm was the most robust method for formulation screening and prediction.

\subsubsection{Application of the PADE}

Perhaps the most useful application of PADE is the formulation screening and prediction. It has been shown that the two parameters $\left(F P F_{\max }\right.$ and $\left.k_{d}\right)$ in Eq. 5-3 can be obtained by correlation of performance data with SET shear stress. It'll be easy to find the optimum shear stress required for efficient deaggregation close to the plateau, yet within the specific resistance limit for patient's inhalation. This new method for heterogeneous dry powder formulation evaluation is significant because it not only describes the formulation performance across the entire shear stress range, but more importantly it has the potential to reveal the mechanism of drug deaggregation considering the surface energy heterogeneity and lateral interaction forces. This can be achieved by increasing the dataset for PADE nonlinear curve fitting of different algebraic equivalent surface adsorption models, and use statistical thermodynamic approach to particle deaggregation studies analogous to those used in adsorption theory.

The analogy of aerosol performance from dry powder formulations with molecular surface adsorption has important implications. Expressions describing surface adsorption were intended to allow elucidation of mechanisms including using the Brunauer classification for reference, lateral interactions, multilayers and capillary condensation.[21] The evolution of the Brunauer Emmett Teller (BET) equation encompassed the Langmuir expression as a special case with the assumptions identified above.[22, 23] Similar 
approaches were described by Giles et al. for solute adsorption at solid surfaces from solution resulting in a range of isotherms equivalent to the Brunauer classification.[16, 17] By using a similar expression for aerosol performance which is limited only by the data available at various shear stresses, from which the hyperbolic curve is derived, it is conceivable that an analogous mechanistic approach to the evaluation of particle-particle (drug-drug, drugexcipient fines), particle-surface (drug-excipient carrier, excipient fines-excipient carrier) interactions might be achieved. Such an approach would have the benefit of describing accurately the magnitude of the interaction and the likely underlying mechanism, based on inflections in FPF with respect to shear that could be probed, subsequently, by controlled experiments (highly engineering particles) or using surface analytical methods. To our knowledge this is the first time that such an approach has been suggested. It is a simple and descriptive method that can find its use in many more practical applications. This theory chapter is served to open a new window for the novel interpretation of aerosol formulations development.

Two examples using PADE are illustrated below. The first example is aimed at comparing the aerosolization performance of SETs and commercial devices. Two commercial devices: Rotahaler ${ }^{\circledR}$ and Aerolizer ${ }^{\circledR}$ were chosen for this study. The second example is an evaluation of selected formulations upon storage. Both examples illustrate the robustness and flexibility of performance evaluation using PADE.

\subsubsection{Commercial devices vs. SETs}

Rotahaler ${ }^{\circledR}(\mathrm{GSK})$ and Aerolizer ${ }^{\circledR}$ (Novartis) are two first generation devices that have long been approved for use in the United States. (see Appendix 1-A). The powder supply for both 
devices is single dose capsule. Their mechanisms of powder fluidization and deaggregation were well-studied using experimental $[24,25]$ and computational fluid dynamics [26, 27] methods. The Rotahaler ${ }^{\circledR}$ is classified as a low specific resistance device $\left(R_{D}=0.036\right.$ $\left.\mathrm{cmH}_{2} \mathrm{O}^{1 / 2} /\left(\mathrm{Lmin}^{-1}\right)\right)$.[24] Its shear stress value at $60 \mathrm{~L} / \mathrm{min}$ was reported to be $2.026 \mathrm{~N} / \mathrm{m}^{2}$.[7] The actuation of Rotahaler ${ }^{\circledR}$ is achieved by mechanically breaking the capsule in half. Carrier-based formulation (Ventolin Rotacaps ${ }^{\circledR}$ ) fluidization from the open capsule is a combination of vibration, impaction and shear force. Particle deaggregation is mainly caused by turbulence promoted by the grid upstream of the mouthpiece. The Aerolizer ${ }^{\circledR}$ has a relatively high specific resistance $\left(R_{D}=0.072 \mathrm{cmH}_{2} \mathrm{O}^{1 / 2} /\left(\mathrm{Lmin}^{-1}\right)\right)$.[26] Its shear stress value at $60 \mathrm{~L} / \mathrm{min}$ airflow rate $\left(8.248 \mathrm{~N} / \mathrm{m}^{2}\right)$ was derived from the pressure drop (see Appendix 5B). During actuation, the capsule in the Aerolizer ${ }^{\circledR}$ is pierced at both ends and the powder emptying occurs primarily by capillary fluidization due to pressure drop across the capsule. Deaggregation is mainly caused by shear force and relative motion. Based on the Poiseuille's law, the airflow conditions in both devices are not fully-developed.

The characteristics of SETs was introduced in Chapter 1, section 1.3.2.2. The SETs have fully developed and well-defined airflow parameters. The principal mechanism of fluidization and deaggregation is the aerodynamic turbulent shear force.[7] The SETs are designed such that the impact of particles due to inertia could be minimized. However, the range of specific resistances provided by SETs encompassed those of commercial DPIs.

The aim of this study was to compare the aerosolization performance of DPIs and SETs using the same carrier-based formulations. Based on the predictive behavior of PADE method across the investigated shear stress range, we hypothesized that if the aerosolization performance of SETs was comparable to that of DPIs, the performance efficiency of DPIs 
could be predicted from the performance data using SETs, i.e. the performance data using DPIs would land on the PADE non-linear regression or linear regression curves generated using SETs.

Following the evaluation of PADE models, the same two formulations: AS_Mann and DSCG_ML-B at $2 \%(\mathrm{w} / \mathrm{w})$ drug concentration were selected. The performance data (ED, $\mathrm{FPF}_{\mathrm{TD}}, \mathrm{PFP}_{\mathrm{ED}}$ ) using SETs were adopted from previous TSLI experiment. The performance of Rotahaler ${ }^{\circledR}$ and Aerolizer ${ }^{\circledR}$ was evaluated in similar conditions (with solenoid switch, 10 $\mathrm{s}$ actuation at $60 \mathrm{~L} / \mathrm{min}, \mathrm{TSLI})$. Two types of size \#3 hard capsules: the gelatin capsule (Capsugel ${ }^{\circledR}$, Greenwood, SC) and HPMC capsule (Vcaps ${ }^{\circledR}$ Plus, Greenwood, SC) were used for the performance studies. As usual, the number of each experiment was randomly assigned (Design Expert 5.09 software, Stat-Ease Corp., MN) and in vitro aerosolization was evaluated in triplicate under ambient condition.

The performance data $\left(E D, F P F_{T D}\right.$, and $\left.F P F_{E D}\right)$ of Rotahaler ${ }^{\circledR}$ and Aerolizer ${ }^{\circledR}$ are shown in Appendix 5-D. The ED values obtained using SETs were generally higher than those obtained using the inhaler devices. The lower $E D$ values using inhaler devices were attributed to the use of capsules and grids in DPIs. Extra energy was required to fluidize the powder by mechanical impaction (Rotahaler $\left.{ }^{\circledR}\right)$ and shear fluidization (Aerolizer $\left.{ }^{\circledR}\right)$. The $E D$ values using Aerolizer ${ }^{\circledR}$ were superior to those of Rotahaler ${ }^{\circledR}$. The influence of different capsules, gelatin or HPMC capsule, to the aerosolization performance was inconclusive because of the limited data obtained. But the data indicated a trend that HPMC capsules resulted in higher ED, whereas gelatin capsules gave higher $F P F$. When the $F P F_{T D}$ and shear stress $\left(\tau_{s}\right)$ of the devices (SETs and DPIs) were correlated, the PADE analysis is depicted in Figure 5-5. The $F P F$ values obtained using DPIs were well-predicted using SETs. For 
AS/Mann formulation, the performance $\left(F P F_{T D}\right)$ data using DPI devices were on the PADE regression curves predicted by SETs. For DSCG/ML-780 formulation, a slightly lower but quite comparable $F P F_{T D}$ values were obtained using DPI devices. This was reasonable because powder fluidization and entrainment from the SETs were not hindered by capsules or grids. The collision on the rigid stainless steel walls of SETs vs. softer and possibly electrostatic charged plastic walls of DPI devices could also explain the slight over-estimated performance efficiency using SETs. However, considering the apparent differences in geometry, dimension, and the mechanisms of particle separation, the prediction of aerosolization performance using SETs was remarkable. The performance using SETs was initially evaluated using Ventolin ${ }^{\circledR}$ (AS-lactose monohydrate) formulation vs. two DPI devices (Rotahaler ${ }^{\circledR}$ and Inhalator $\left.{ }^{\circledR}\right)$.[7] Here, the predictive capability of SETs was further confirmed by variation in four different factors including drugs (AS and DSCG), carriers (lactose monohydrate and D-mannitol), DPI devices (Rotahaler ${ }^{\circledR}$ and Aerolizer ${ }^{\circledR}$ ), and capsules (gelatin and HPMC).

The intent of this performance study was to challenge the predictive capability of SETs as device independent screening tools. Undoubtedly, in the final product, the DPI devices with different principal mechanisms of particle separation would be employed. The exact mechanisms of particle separation of specific DPIs, due to their turbulent nature, were still unclear. Many literatures were focused on the impaction and momentum transfer mechanisms (mass or volume related contribution) for which many devices were designed. However, the important role of aerodynamic shear to the aerosolization performance was not emphasized or simply ignored. Nevertheless, it is reasonable to suggest that particles which can be stripped by aerodynamic shear may more readily be removed under the influence of 
volume or mass related contribution. The SETs are excellent tools for the formulation optimization and prediction during the early stage of DPI development.

\subsubsection{Storage effect}

The pharmaceutical processing may inevitably induce surface imperfection and molecular disorder in the dry powder formulations. They are susceptible to change at elevated relative humidity $(\mathrm{RH})$ or temperature because there is a tendency for the transformation of these formulations to the thermodynamically most favorable state. Many literatures have reported the influence of storage humidity on the aerosolization performance of dry powder formulations.[28, 29] The performance change, often deterioration, could result in insufficient dose and under-treatment. The influence by RH could be effectively minimized by using individually sealed blisters or capsules of each dose. However, in some formulations, particularly those containing steroids (e.g. Seretide Diskus: salmeterol/fluticasone propionate), the performance deterioration could occur even at wellcontrolled storage condition. The exact cause of the change in performance is still unknown, presumably because of relaxation and phase change at molecular level.

The intent of this study was to examine if the PADE method could still be used after formulation storage, and if the regression analysis would still furnish good data fit. Two formulations DSCG-ML780 and FP-Treh were chosen for this study. Since the earlier studies (Chapter 3 and 4), they have been stored in sealed glass jars which have in turn, been stored in desiccators (Drierite ${ }^{\circledR} /$ Indicating Drierite ${ }^{\circledR}$ ) under ambient temperature and pressure for about a year. From the previous studies (Chapter 3), DSCG-ML780 was known to give the best performance efficiency (highest $\mathrm{FPF}_{\mathrm{TD}}$ ) among all assessed formulations. DSCG is an 
interesting molecule that can reversibly sorb and librate water to form nonstoichiometric hydrates.[30, 31] It was reported previously that elevated RH may greatly influence the aerosolization performance of a DSCG-Pharmatose (Lactose monohydrate) formulation.[28] However, the performance of this formulation at well-controlled storage conditions was not studied. The other formulation, FP-Treh was chosen for two reasons. FP is a steroid known to affect performance upon storage.[29] Treh is a dihydrate that may partially lose bound water and preferentially turn to its $\alpha$-polymorph at low RH.[32] The thermal analysis using DSC also showed that Treh had a low dehydration temperature (onset $\sim 86{ }^{\circ} \mathrm{C}$ and peak $\sim 93{ }^{\circ} \mathrm{C}$ ) (Figure 4-4). The morphology change and dehydration may lead to variations in formulation performance.

The performance data before and after storage are shown in Appendix 3-A1, 5-B, and 5-E, respectively. (Some data were adopted from early studies). The PADE regression analyses are depicted in Figure 5-6. The result indicated that the performance of DSCGML780 did not have appreciable change upon storage over a year range. However, the aerosolization of FP-Treh became less efficient. The value of $F P F_{\max }$ (plateau region in Figure 5-6D) decreased upon storage, and followed a new PADE curve. The shear stress dependent region increased (indicated as a slower rise in the graph) as well. This implied that the formulation reproducibility at different shear stress decreased after storage. Both PADE nonlinear and linear regression gave good correlation results. We assumed that the performance of other formulations after storage could also be predicted using PADE. Based on this assumption and the good correlation results obtained so far, we concluded a rule of thumb that the PADE linear regression divided the graph into two regions, any further 
performance data that landed on the right-lower region had better performance efficiency, whereas any performance data that landed on the left-upper region had poorer performance.

\subsection{Conclusion}

In the current literature, attention was focused on inertial separation forces (volume or mass related: collision, rotation, vibration) and "torque" forces, while much less emphasis was put on the fundamental aerodynamic shear stress for particle separation. Using SETs in which particle separation occurs in response to shear stress, a novel method that correlates the pharmaceutical dry powder formulation performance with this airflow parameter for formulation screening and prediction in the entire shear stress range has been proposed. Particles which can be stripped by shear forces may more readily be removed under the influence of impaction and momentum transfer, from which it can be inferred that these formulation performance studies may extrapolate qualitatively (e.g. as a rank order) to drug product (device and formulation) performance. Two examples showed the robustness of PADE in formulation prediction across a defined shear stress range. PADE, as a theoretical method analogous to the Langmuir adsorption isotherm, also has the potential to allow a statistical thermodynamic approach to particle detachment utilizing a variety of expression used in a plethora surface adsorption theories, such as Freundlich, Sips, Toth, Volmer, Fowler-Guggenheim, Hill-deBoer, and many more. It may serve as a link between macroscopic statistical thermodynamics and microscopic individual particle deaggregation. This methodology gives an entirely new perspective for the rationale design of DPI formulation for pulmonary drug delivery. The current studies leading to PADE was based on carrier-based formulation systems. The dry powder aerosolization performance is a very 
complex process. The heterogeneity of particulate systems, lateral interaction, the nonideality of the bulk, and the inconsistent airflow conditions in inhaler devices, all influence the macroscopic aerosol formulation performance. This complex deaggregation process can now simply be described by adapting surface adsorption theories and fitting the shear displacement using SETs. The effect of different formulation performance and the heterogeneity can be discussed in terms of theoretical or empirical surface adsorption models and measured experimentally to describe the aerosol performance across the entire shear stress range. It is suitable for accurate prediction of deaggregation mechanisms for real pharmaceutical formulations having surface energy heterogeneity and lateral interaction forces. It may also be applied for the studies such as the debatable ternary formulation deaggregation ("active" site vs deagglomeration), formulation stability and storage prediction, the influence of engineered particles such as porous carriers to the formulation performance. 
Table 5-1. Results of PADE Linear-regression Using Four Different Linearization Methods of PADE (Langmuir isotherm equivalent) for Performance Studies*

\begin{tabular}{|c|c|c|c|c|c|c|c|}
\hline \multirow{2}{*}{\multicolumn{2}{|c|}{ Formulation }} & \multicolumn{3}{|c|}{ Langmuir linear regression } & \multirow{3}{*}{$\begin{array}{c}\begin{array}{c}\text { Scatchard linear } \\
\text { regression }\end{array} \\
\boldsymbol{R}^{2} \\
0.8203\end{array}$} & \multirow{3}{*}{$\begin{array}{c}\begin{array}{c}\text { Eadie Hofstee } \\
\text { linear regression }\end{array} \\
\boldsymbol{R}^{2} \\
0.8203\end{array}$} & \multirow{3}{*}{$\begin{array}{c}\begin{array}{c}\text { Lineweaver-Burk } \\
\text { linear regression }\end{array} \\
\boldsymbol{R}^{2} \\
0.9137\end{array}$} \\
\hline & & \multirow{2}{*}{$\begin{array}{c}F P F_{\text {max }} \\
(\%)\end{array}$} & \multirow{2}{*}{$\begin{array}{c}\begin{array}{c}K \\
\left(m^{2} / N\right)\end{array} \\
0.81 \\
\end{array}$} & \multirow{2}{*}{$\begin{array}{c}\boldsymbol{R}^{2} \\
0.9937\end{array}$} & & & \\
\hline \multirow{6}{*}{$\stackrel{\pi}{<}$} & SV-A & & & & & & \\
\hline & SV-B & 16.9 & 0.59 & 0.9804 & 0.6888 & 0.6888 & 0.9172 \\
\hline & ML-A & 45.7 & 1.01 & 0.9986 & 0.9228 & 0.9228 & 0.9747 \\
\hline & ML-B & 36.6 & 0.85 & 0.9912 & 0.8224 & 0.8224 & 0.9442 \\
\hline & Treh & 14.3 & 0.59 & 0.9918 & 0.8262 & 0.8262 & 0.9391 \\
\hline & Mann & 11.6 & 0.73 & 0.9872 & 0.8070 & 0.8070 & 0.9100 \\
\hline \multirow{6}{*}{ 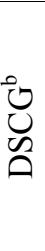 } & SV-A & 33.6 & 5.77 & 0.9995 & 0.8693 & 0.8693 & 0.9400 \\
\hline & SV-B & 35.3 & 7.81 & 0.9996 & 0.8823 & 0.8823 & 0.9249 \\
\hline & ML-A & 45.8 & 1.13 & 0.9997 & 0.9854 & 0.9854 & 0.9983 \\
\hline & ML-B & 48.8 & 1.22 & 0.9998 & 0.9911 & 0.9911 & 0.9976 \\
\hline & Treh & 15.4 & - & 0.9968 & 0.8366 & 0.8366 & 0.8608 \\
\hline & Mann & 23.3 & 0.84 & 0.9955 & 0.9027 & 0.9027 & 0.9832 \\
\hline \multirow{6}{*}{$\stackrel{m}{a}$} & SV-A & 31.4 & - & 0.9997 & 0.3453 & 0.3453 & 0.3881 \\
\hline & SV-B & 28.7 & 42.74 & 0.9956 & 0.0000 & 0.0000 & 0.0007 \\
\hline & ML-A & 32.2 & 18.65 & 0.9995 & 0.9226 & 0.9226 & 0.9560 \\
\hline & ML-B & 37.7 & 2.00 & 0.9981 & 0.8211 & 0.8211 & 0.8985 \\
\hline & Treh & 16.1 & 1.05 & 0.9909 & 0.8641 & 0.8641 & 0.9876 \\
\hline & Mann & 9.9 & 1.33 & 0.9710 & 0.4555 & 0.4555 & 0.9684 \\
\hline \multirow{6}{*}{$\overrightarrow{\vec{a}_{I}}$} & SV-A & 15.2 & 6.01 & 0.9978 & 0.2588 & 0.2588 & 0.4300 \\
\hline & SV-B & 15.9 & 3.43 & 0.9999 & 0.9862 & 0.9862 & 0.9912 \\
\hline & ML-A & 21.4 & 1.25 & 0.9906 & 0.1468 & 0.1468 & 0.1252 \\
\hline & ML-B & 24.3 & 10.16 & 0.9995 & 0.6102 & 0.6102 & 0.4825 \\
\hline & Treh & 9.1 & 1.04 & 0.9274 & 0.0106 & 0.1783 & 0.0066 \\
\hline & Mann & 12.5 & - & 0.9964 & 0.5756 & 0.5756 & 0.7440 \\
\hline
\end{tabular}

* The correlation of $\boldsymbol{F P F}_{\boldsymbol{T D}}(n=3)$ and SET shear stress $\boldsymbol{\tau}_{\boldsymbol{s}}$ of twenty-four formulations using four different linear regression models. Based on the langmuir linear regression model:

a. Rank order $\left(F P F_{\max }\right)$ of AS formulation:

b. Rank order $\left(F P F_{\max }\right)$ of DSCG formulation:

c. Rank order $\left(F P F_{\max }\right)$ of IPB formulation:

d. Rank order $\left(F P F_{\max }\right)$ of FP formulation:
ML-A $>$ ML-B $>$ SV-B $>$ SV-A $>$ Treh $>$ Mann

ML-B $>$ ML-A $>$ SV-B $>$ SV-A $>$ Mann $>$ Treh

ML-B $>$ ML-A $>$ SV-A $>$ SV-B $>$ Treh $>$ Mann

ML-B $>$ ML-A $>$ SV-B $>$ SV-A $>$ Mann $>$ Treh 
Table 5-2. A Comparison of Surface Adsorption, Molecular/Colloidal Assembly, and Aerosol Dispersion (Analogy and Contrast) [10-13]

\begin{tabular}{|c|c|c|}
\hline 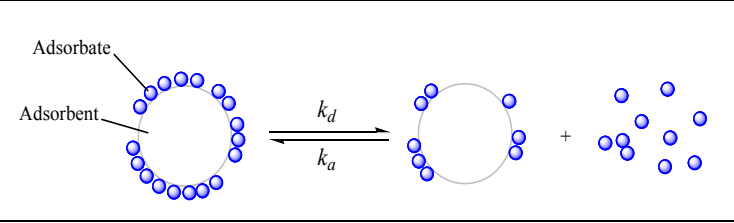 & 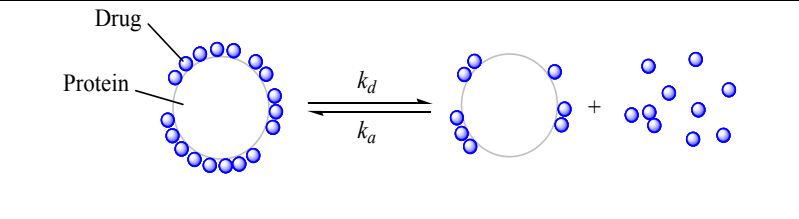 & 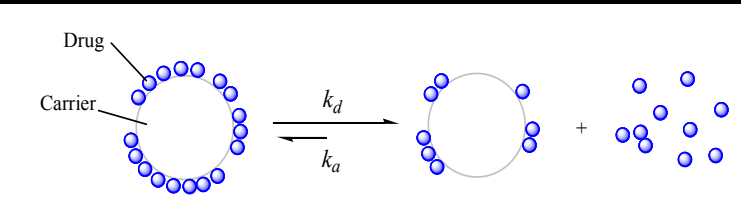 \\
\hline Adsorption $\leftarrow \rightarrow$ Desorption & Association (binding) $\leftarrow \rightarrow$ Dissociation & Adhesion $\leftarrow \rightarrow$ Deaggregation \\
\hline$\Gamma=\frac{\Gamma_{\max } K_{L} P}{1+K_{L} P} \quad$ (Langmuir isotherm) & $r=\frac{v K\left[D_{f}\right]}{1+K\left[D_{f}\right]}$ (Protein binding equilibria) & $F P F=\frac{F P F_{\max } k_{d} \tau_{s}}{1+k_{d} \tau_{s}} \quad$ (Deaggregation equation) \\
\hline $\begin{array}{l}\Gamma: \text { Amount adsorbed } \\
\Gamma_{\max }: \text { maximum adsorption to monolayer coverage } \\
\boldsymbol{K}_{\boldsymbol{L}}: \text { Langmuir adsorption constant } \\
\boldsymbol{P}: \text { gaseous partial pressure or solute concentration } \\
\end{array}$ & $\begin{array}{l}r: \text { amount of drug bound per amount of protein } \\
v: \text { number of independent binding sites } \\
K: \text { association constant } \\
{\left[D_{f}\right]: \text { concentration of free drug (ligand) }} \\
\end{array}$ & $\begin{array}{l}\boldsymbol{F P F}: \text { fine particle fraction below certain cutoff size } \\
\boldsymbol{F P F} \boldsymbol{F}_{\text {max }}: \text { maximum achievable fine particle fraction } \\
\boldsymbol{k}_{d}: \text { deaggregation constant (overcome adhesion) } \\
\tau_{s}: \text { viscous shear stress }\end{array}$ \\
\hline \multicolumn{3}{|c|}{ SIMILARITY } \\
\hline \multicolumn{3}{|c|}{ Phenomena governed by the same fundamental forces: van der Waals, electrostatic, capillary interactions, hydrogen bonding } \\
\hline \multicolumn{3}{|c|}{ A result of surface energy, capable of being described as interfacial interaction, the same algebraic expression } \\
\hline \multicolumn{3}{|c|}{ Interfacial phenomena. Interfacial driving force: gaseous partial pressure $\left(\mathrm{N} / \mathrm{m}^{2}\right)$, solute concentration $(\mathrm{mg} / \mathrm{g})$, shear stress $\left(\mathrm{N} / \mathrm{m}^{2}\right)$} \\
\hline \multicolumn{3}{|c|}{ DIFFERENCE } \\
\hline Gas-Solid or Liquid-Solid Interface & Liquid-Liquid or Liquid-Solid Interface & Solid-Solid Interface \\
\hline Molecular adsorption/desorption; & Colloidal aggregation/deaggregation & Particulate adhesion/deaggregation \\
\hline Statistical thermodynamics or kinetic approach & Derived from binding equilibria & Possibly the statistical thermodynamic approach \\
\hline Equilibrium (time independent) & Equilibrium (time independent) & Time dependent kinetic process \\
\hline System volume \& boundary condition remain constant & System volume \& boundary condition remain constant & Rapid system volume expansion, net gain in entropy \\
\hline \multirow[t]{2}{*}{ Monolayer surface coverage } & Number of independent binding sites & Maximum achievable fine particle fraction \\
\hline & HETEROGENEITY & \\
\hline Adsorbent geometric and energetic feature & Affinity distribution, binding site dependency & Carrier geometric and energetic characteristics \\
\hline Adsorbate size, shape and conformation & Different classes of binding sites & Drug geometric and energetic characteristics \\
\hline Lateral interaction & Drug-drug interaction, agglomeration & Drug-drug cohesion, agglomeration \\
\hline \multicolumn{3}{|c|}{ EXAMPLE } \\
\hline $\begin{array}{l}\text { Chemosorption (irreversible), } \\
\text { Physical adsorption (reversible) }\end{array}$ & $\begin{array}{c}\text { Protein binding (reversible), } \\
\text { drug-drug interaction (reversible or irreversible) } \\
\text { Enzyme kinetics (irreversible) }\end{array}$ & Dry powder Aerosol dispersion (irreversible) \\
\hline
\end{tabular}


Table 5-3. A Schematic Comparison of Algebraic Equivalent Langmuir* and Freundlich Expression for TSLI Formulation Performance. (PADE non-linear regression analyses).

\begin{tabular}{|c|c|c|c|}
\hline \multicolumn{2}{|c|}{ PADE } & Langmuir* & Freundlich \\
\hline \multicolumn{2}{|c|}{ Expression } & $F P F=\frac{F P F_{\max } k_{L} \tau_{s}}{1+k_{L} \tau_{s}}$ & $F P F=k_{F} \tau_{s}{ }^{1 / n}$ \\
\hline 2 & SV-A & $\begin{array}{l}F P F_{\max }=14.49 \\
k_{L}=1.31 \\
R^{2}=0.9739 \\
\text { Adj. } R^{2}=0.9652\end{array}$ & $\begin{array}{l}n=4.46 \\
k_{F}=8.27 \\
R^{2}=1.0000 \\
\text { Adj. } R^{2}=1.0000\end{array}$ \\
\hline \& & SV-B & $\begin{array}{l}F P F_{\max }=16.55 \\
k_{L}=0.75 \\
R^{2}=0.9362 \\
\text { Adj. } R^{2}=0.9149\end{array}$ & $\begin{array}{l}n=3.37 \\
k_{F}=7.51 \\
R^{2}=0.9882 \\
\text { Adj. } R^{2}=0.9843\end{array}$ \\
\hline 2 & ML-A & $\begin{array}{l}F P F_{\max }=38.54 \\
k_{L}=0.76 \\
R^{2}=0.9691 \\
\text { Adj. } R^{2}=0.9588\end{array}$ & $\begin{array}{l}n=4.62 \\
k_{F}=25.07 \\
R^{2}=0.9957 \\
\text { Adj. } R^{2}=0.9943\end{array}$ \\
\hline 4 & ML-B & $\begin{array}{l}F P F_{\max }=43.51 \\
k_{L}=1.32 \\
R^{2}=0.9893 \\
\text { Adj. } R^{2}=0.9858\end{array}$ & $\begin{array}{l}n=3.67 \\
k_{F}=18.08 \\
R^{2}=0.9371 \\
\text { Adj. } R^{2}=0.9161\end{array}$ \\
\hline 4 & Treh & $\begin{array}{l}F P F_{\max }=13.30 \\
k_{L}=0.78 \\
R^{2}=0.9740 \\
\text { Adj. } R^{2}=0.9654\end{array}$ & $\begin{array}{l}n=3.53 \\
k_{F}=6.21 \\
R^{2}=0.9996 \\
\text { Adj. } R^{2}=0.9995\end{array}$ \\
\hline 4 & Mann & $\begin{array}{l}F P F_{\text {max }}=10.49 \\
k_{L}=1.25 \\
R^{2}=0.9653 \\
\text { Adj. } R^{2}=0.9537\end{array}$ & $\begin{array}{l}n=4.30 \\
k_{F}=5.86 \\
R^{2}=0.9928 \\
\text { Adj. } R^{2}=0.9905\end{array}$ \\
\hline 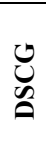 & SV-A & $\begin{array}{l}F P F_{\text {max }}=35.59 \\
k_{L}=3.00 \\
R^{2}=0.9896 \\
\text { Adj. } R^{2}=0.9861\end{array}$ & $\begin{array}{l}n=9.10 \\
k_{F}=26.83 \\
R^{2}=0.9603 \\
\text { Adj. } R^{2}=0.9471\end{array}$ \\
\hline $\begin{array}{l}U_{0} \\
\text { Å }\end{array}$ & SV-B & $\begin{array}{l}F P F_{\text {max }}=37.40 \\
k_{L}=3.75 \\
R^{2}=0.9927 \\
\text { Adj. } R^{2}=0.9902\end{array}$ & $\begin{array}{l}n=10.78 \\
k_{F}=29.55 \\
R^{2}=0.9714 \\
\text { Adj. } R^{2}=0.9618\end{array}$ \\
\hline $\begin{array}{l}\text { Un } \\
\text { ñ }\end{array}$ & ML-A & $\begin{array}{l}F P F_{\max }=44.78 \\
k_{L}=1.32 \\
R^{2}=0.9978 \\
\text { Adj. } R^{2}=0.9970\end{array}$ & $\begin{array}{l}n=4.73 \\
k_{F}=25.98 \\
R^{2}=0.9857 \\
\text { Adj. } R^{2}=0.9809\end{array}$ \\
\hline ڤ્ & ML-B & $\begin{array}{l}F P F_{\max }=48.62 \\
k_{L}=1.30 \\
R^{2}=0.9987 \\
\text { Adj. } R^{2}=0.9983\end{array}$ & $\begin{array}{l}n=4.75 \\
k_{F}=28.12 \\
R^{2}=0.9739 \\
\text { Adj. } R^{2}=0.9652\end{array}$ \\
\hline Uి & Treh & $\begin{array}{l}F P F_{\max }=18.06 \\
k_{L}=2.87 \\
R^{2}=0.9847 \\
\text { Adj. } R^{2}=0.9797\end{array}$ & $\begin{array}{l}n=9.29 \\
k_{F}=13.58 \\
R^{2}=0.9461 \\
\text { Adj. } R^{2}=0.9281\end{array}$ \\
\hline U્ & Mann & $\begin{array}{l}F P F_{\max }=22.30 \\
k_{L}=1.13 \\
R^{2}=0.9811 \\
\text { Adj. } R^{2}=0.9747\end{array}$ & $\begin{array}{l}n=4.20 \\
k_{F}=12.08 \\
R^{2}=0.9891 \\
\text { Adj. } R^{2}=0.9854\end{array}$ \\
\hline
\end{tabular}

\begin{tabular}{|c|c|c|c|}
\hline \multicolumn{2}{|c|}{ PADE } & Langmuir* & Freundlich \\
\hline \multicolumn{2}{|c|}{ Expression } & $F P F=\frac{F P F_{\max } k_{L} \tau_{s}}{1+k_{x} \tau}$ & $F P F=k_{F} \tau_{s}{ }^{1 / n}$ \\
\hline 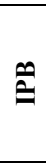 & SV-A & $\begin{array}{l}F P F_{\max }=32.99 \\
k_{L}=25.22 \\
R^{2}=0.9954 \\
\text { Adj. } R^{2}=0.9939\end{array}$ & $\begin{array}{l}n=110.40 \\
k_{F}=31.93 \\
R^{2}=0.9933 \\
\text { Adj. } R^{2}=0.9911\end{array}$ \\
\hline 急 & SV-B & $\begin{array}{l}F P F_{\max }=29.51 \\
k_{L}=80.02 \\
R^{2}=0.9438 \\
\text { Adj. } R^{2}=0.9250\end{array}$ & $\begin{array}{l}n=- \\
k_{F}=29.3 \\
R^{2}=0.9435 \\
\text { Adj. } R^{2}=0.9246\end{array}$ \\
\hline 惫 & ML-A & $\begin{array}{l}F P F_{\max }=34.02 \\
k_{L}=5.26 \\
R^{2}=0.9967 \\
\text { Adj. } R^{2}=0.9955\end{array}$ & $\begin{array}{l}n=14.29 \\
k_{F}=28.53 \\
R^{2}=0.9840 \\
\text { Adj. } R^{2}=0.9786\end{array}$ \\
\hline 急 & ML-B & $\begin{array}{l}F P F_{\max }=37.56 \\
k_{L}=2.11 \\
R^{2}=0.9806 \\
\text { Adj. } R^{2}=0.9741\end{array}$ & $\begin{array}{l}n=6.39 \\
k_{F}=25.45 \\
R^{2}=0.9746 \\
\text { Adj. } R^{2}=0.9662\end{array}$ \\
\hline 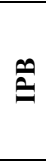 & Treh & $\begin{array}{l}F P F_{\max }=17.62 \\
k_{L}=0.79 \\
R^{2}=0.9716 \\
\text { Adj. } R^{2}=0.9621\end{array}$ & $\begin{array}{l}n=3.77 \\
k_{F}=8.40 \\
R^{2}=0.9038 \\
\text { Adj. } R^{2}=0.8717\end{array}$ \\
\hline$\stackrel{\theta}{\hat{\theta}}$ & Mann & $\begin{array}{l}F P F_{\max }=11.56 \\
k_{L}=0.91 \\
R^{2}=0.8991 \\
\text { Adj. } R^{2}=0.8655\end{array}$ & $\begin{array}{l}n=4.28 \\
k_{F}=5.94 \\
R^{2}=0.7870 \\
\text { Adj. } R^{2}=0.7159\end{array}$ \\
\hline 产 & SV-A & $\begin{array}{l}F P F_{\max }=14.95 \\
k_{L}=14.84 \\
R^{2}=0.9823 \\
\text { Adj. } R^{2}=0.9764\end{array}$ & $\begin{array}{l}n=35.12 \\
k_{F}=13.95 \\
R^{2}=0.9802 \\
\text { Adj. } R^{2}=0.9736\end{array}$ \\
\hline 产 & SV-B & $\begin{array}{l}F P F_{\max }=16.04 \\
k_{L}=3.37 \\
R^{2}=0.9993 \\
\text { Adj. } R^{2}=0.9990\end{array}$ & $\begin{array}{l}n=9.16 \\
k_{F}=12.28 \\
R^{2}=0.9867 \\
\text { Adj. } R^{2}=0.9822\end{array}$ \\
\hline$\frac{1}{x}$ & ML-A & $\begin{array}{l}F P F_{\max }=18.57 \\
k_{L}=5.19 \\
R^{2}=0.9000 \\
\text { Adj. } R^{2}=0.8667\end{array}$ & $\begin{array}{l}n=8.46 \\
k_{F}=14.68 \\
R^{2}=0.9368 \\
\text { Adj. } R^{2}=0.9157\end{array}$ \\
\hline 宝 & ML-B & $\begin{array}{l}F P F_{\text {max }}=24.20 \\
k_{L}=14.33 \\
R^{2}=0.9954 \\
\text { Adj. } R^{2}=0.9939\end{array}$ & $\begin{array}{l}n=27.39 \\
k_{F}=22.34 \\
R^{2}=0.9962 \\
\text { Adj. } R^{2}=0.9950\end{array}$ \\
\hline 空 & Treh & $\begin{array}{l}F P F_{\text {max }}=7.99 \\
k_{L}=10.70 \\
R^{2}=0.7987 \\
\text { Adj. } R^{2}=0.7316\end{array}$ & $\begin{array}{l}n=18.76 \\
k_{F}=7.15 \\
R^{2}=0.8014 \\
\text { Adj. } R^{2}=0.7352\end{array}$ \\
\hline 空 & Mann & $\begin{array}{l}F P F_{\max }=14.43 \\
k_{L}=4.35 \\
R^{2}=0.9657 \\
\text { Adj. } R^{2}=0.9543\end{array}$ & $\begin{array}{l}n=15.43 \\
k_{F}=11.96 \\
R^{2}=0.9318 \\
\text { Adj. } R^{2}=0.9090\end{array}$ \\
\hline
\end{tabular}


Table 5-4. Dimensions of the Additional SETs and Their Corresponding Airflow Parameters.

\begin{tabular}{|c|c|c|c|c|c|c|c|}
\hline \multirow{3}{*}{ SET } & \multicolumn{6}{|c|}{ Dimensions (mm)** } & \multicolumn{2}{c|}{ Airflow parameters } \\
\cline { 2 - 8 } & $\begin{array}{c}\text { External } \\
\text { outlet length } \\
(\boldsymbol{a})\end{array}$ & $\begin{array}{c}\text { Inlet } \\
\text { length } \\
(\boldsymbol{b})\end{array}$ & $\begin{array}{c}\text { Internal } \\
\text { outlet length } \\
(\boldsymbol{c})\end{array}$ & $\begin{array}{c}\text { Inlet outer } \\
\text { diameter } \\
(\boldsymbol{e})\end{array}$ & $\begin{array}{c}\text { Inlet inner } \\
\text { diameter } \\
(\boldsymbol{f})\end{array}$ & $\begin{array}{c}\text { Reynold's } \\
\text { No., } \boldsymbol{R} \boldsymbol{e},\end{array}$ & $\begin{array}{c}\text { Shear Stress } \\
\boldsymbol{\tau}_{\boldsymbol{s}}\left(\mathbf{N} / \mathbf{m}^{2}\right)\end{array}$ \\
\hline A1* $^{*}$ & 144 & 300 & 134 & 7.0 & 5.2 & 16196 & 8.742 \\
\hline B1 $^{*}$ & 133 & 300 & 123 & 8.5 & 7.0 & 12059 & 2.775 \\
\hline C1 $^{\#}$ & 121 & 600 & 111 & 10.1 & 8.3 & 10126 & 1.412 \\
\hline
\end{tabular}

* The SET-A1 and SET-B1 use peripherally-located dosage table

\# The SET-C1 uses centrally-located dosage table.

** The powder dosage length $(d)$ is set at $10 \mathrm{~mm}$. 
Table 5-5. A Comparison of PADE Models Using Algebraic Equivalent Surface Adsorption Expressions for TSLI Formulation Performance (Using 7 SETs, Correlated by PADE nonlinear regression analyses including algebraic equivalent Langmuir isotherm, Freundlich, and Sips expressions.)

\begin{tabular}{|c|c|c|c|c|}
\hline & \multicolumn{3}{|c|}{ PADE non-linear regression } \\
\hline & & \multirow{2}{*}{$\begin{array}{c}\text { Langmuir } \\
F P F=\frac{F P F_{\max } k_{L} \tau_{s}}{1+k_{L} \tau_{s}}\end{array}$} & \multirow{2}{*}{$\begin{array}{l}\text { Freundlich } \\
F P F=k_{F} \tau_{s}{ }^{1 / n}\end{array}$} & Sips \\
\hline & & & & $F P F=F P F_{\max }^{\prime} \frac{\left(k_{S} \tau_{s}\right)^{1 / n^{\prime}}}{1+\left(k_{S} \tau_{s}\right)^{1 / n}}$ \\
\hline \multicolumn{2}{|c|}{ Parameters } & $F P F_{\max }, k_{L}$ & $n, k_{F}$ & $F P F_{\text {max }}^{\prime}, n^{\prime}, k_{S}$ \\
\hline \multirow{2}{*}{ 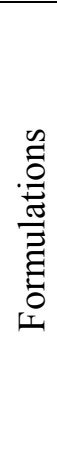 } & $\begin{array}{ll}0 & n \\
0 & 1 \\
0 & 1 \\
0 & 1\end{array}$ & $\begin{array}{l}F P F_{\max }=46.51 \\
k_{L}=1.19 \\
- \\
R^{2}=0.9651 \\
\text { Adj. } R^{2}=0.9593\end{array}$ & $\begin{array}{l}- \\
k_{F}=26.76 \\
n=4.64 \\
R^{2}=0.9610 \\
\text { Adj. } R^{2}=0.9545\end{array}$ & $\begin{array}{l}F P F_{\text {max }}^{\prime}=53.72 \\
k_{S}=0.89 \\
n^{\prime}=1.50 \\
R^{2}=0.9698 \\
\text { Adj. } R^{2}=0.9578\end{array}$ \\
\hline & ऍ & $\begin{array}{l}F P F_{\max }=10.95 \\
k_{L}=0.90 \\
- \\
R^{2}=0.9197 \\
\text { Adj. } R^{2}=0.9064\end{array}$ & $\begin{array}{l}- \\
k_{F}=5.58 \\
n=3.84 \\
R^{2}=0.9682 \\
\text { Adj. } R^{2}=0.9629\end{array}$ & $\begin{array}{l}- \\
- \\
n^{\prime}=12.2 \\
R^{2}=0.9701 \\
\text { Adj. } R^{2}=0.9581\end{array}$ \\
\hline
\end{tabular}


Figure 5-1. PADE (Langmuir equivalent expression) and its linear transformation, where $\boldsymbol{k}_{\boldsymbol{d}}$ is the deaggregation constant $\left(\mathrm{m}^{2} / \mathrm{N}\right) ; \boldsymbol{\tau}_{s}$ is the viscous shear stress of SETs $\left(\mathrm{N} / \mathrm{m}^{2}\right) ; \boldsymbol{F P F}$ is fine particle fraction at certain cutoff size (\%); $\boldsymbol{F P F}_{\max }$ is the maximum achievable fine particle fraction (\%).

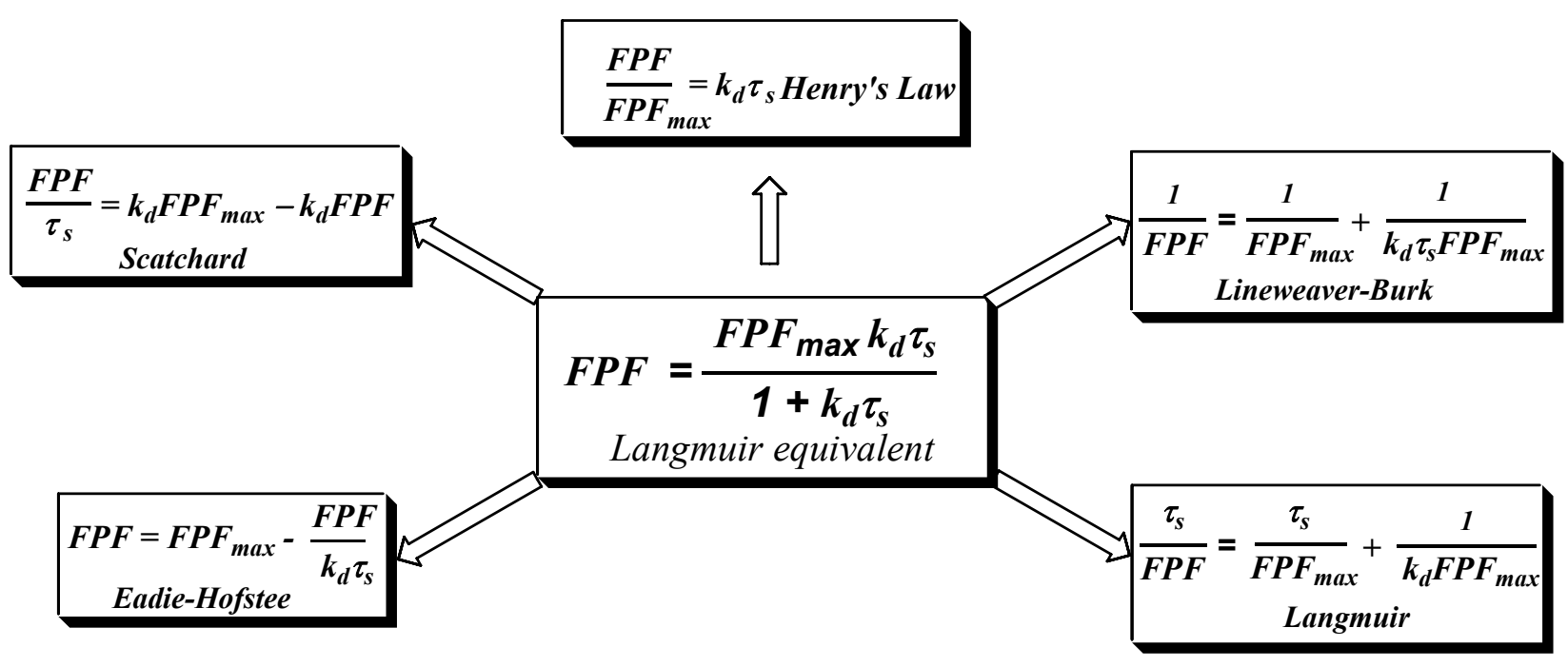


Figure 5-2. The relationship between $\boldsymbol{F P F}_{T D}$ and SET shear stress $\left(\boldsymbol{\tau}_{\boldsymbol{s}}\right)$ by TSLI experiment. PADE (Langmuir isotherm equivalent) linear regression analyses: (A) Albuterol sulfate formulations; (B) Disodium cromoglycate formulations; (C) Ipratropium bromide monohydrate formulations; (D) Fluticasone propionate formulations. All formulations were at $2 \% \mathrm{w} / \mathrm{w}$ drug concentration. SV: Sieved lactose monohydrate; ML: Milled lactose monohydrate; Treh: D-Trehalose dihydrate; Mann: D-Mannitol.
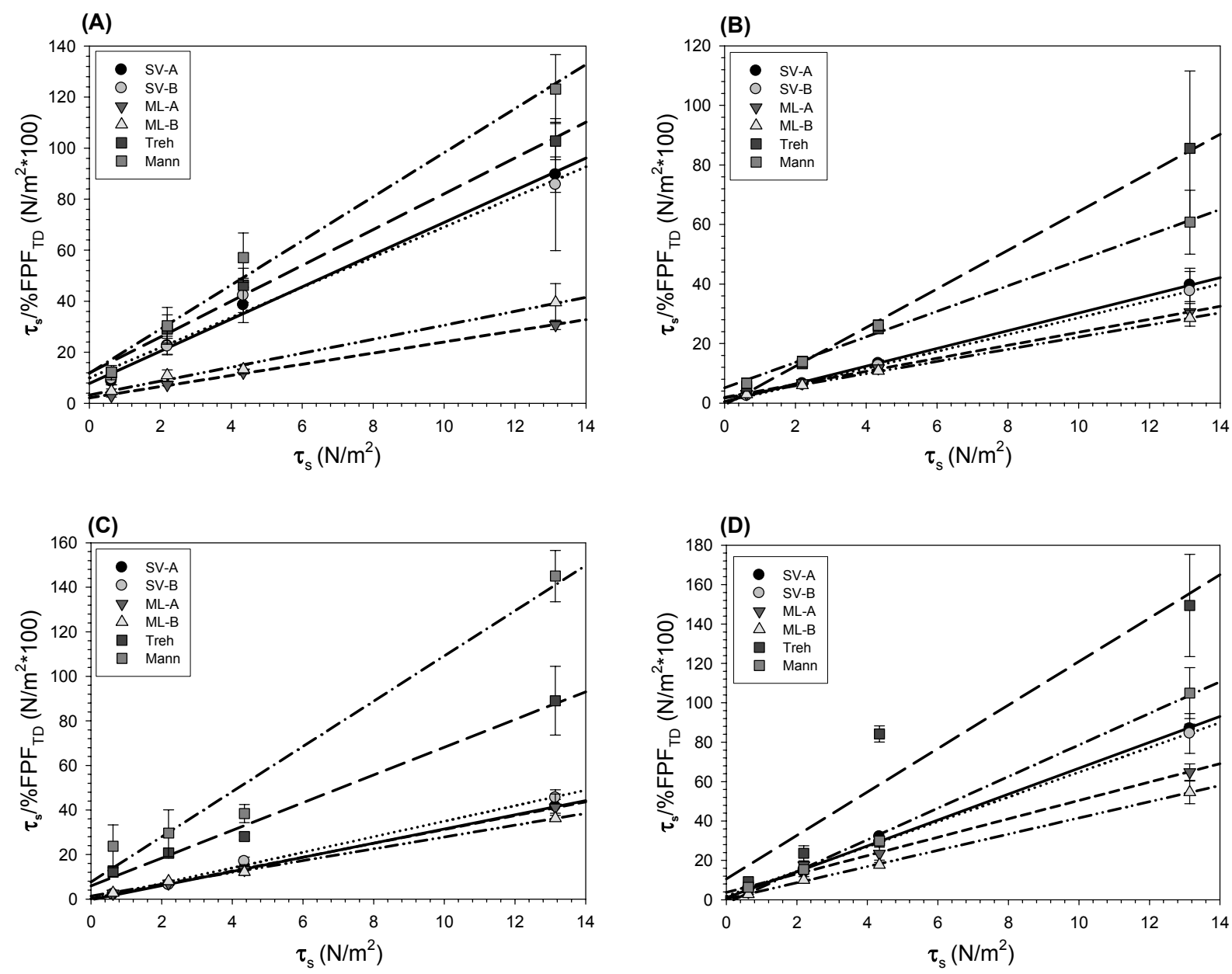
Figure 5-3. TSLI experimental dataset of (A) DSCG/ML-B and (B) AS/Mann formulation using additional SETs, and correlation using PADE non-linear regression: algebraic equivalent of surface adsorption expressions including Langmuir isotherm, Freundlich, and Sips equivalent.

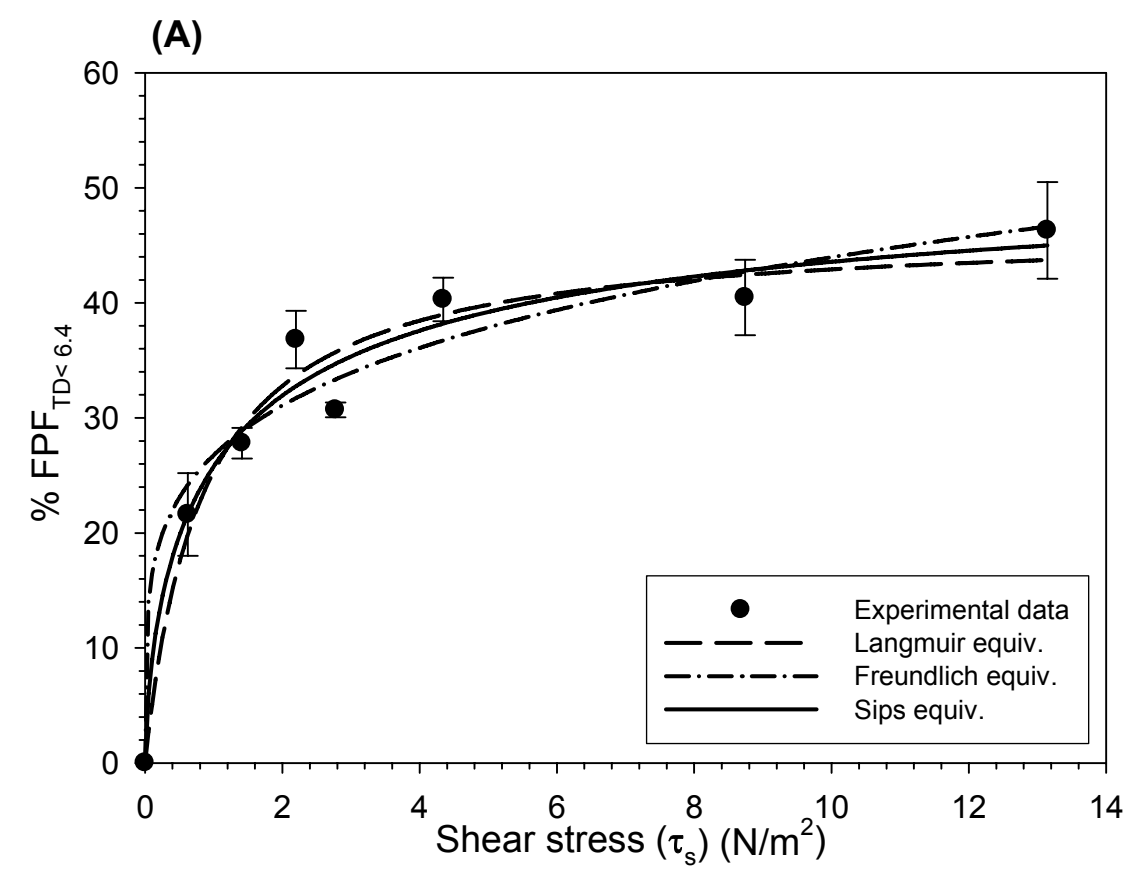

(B)

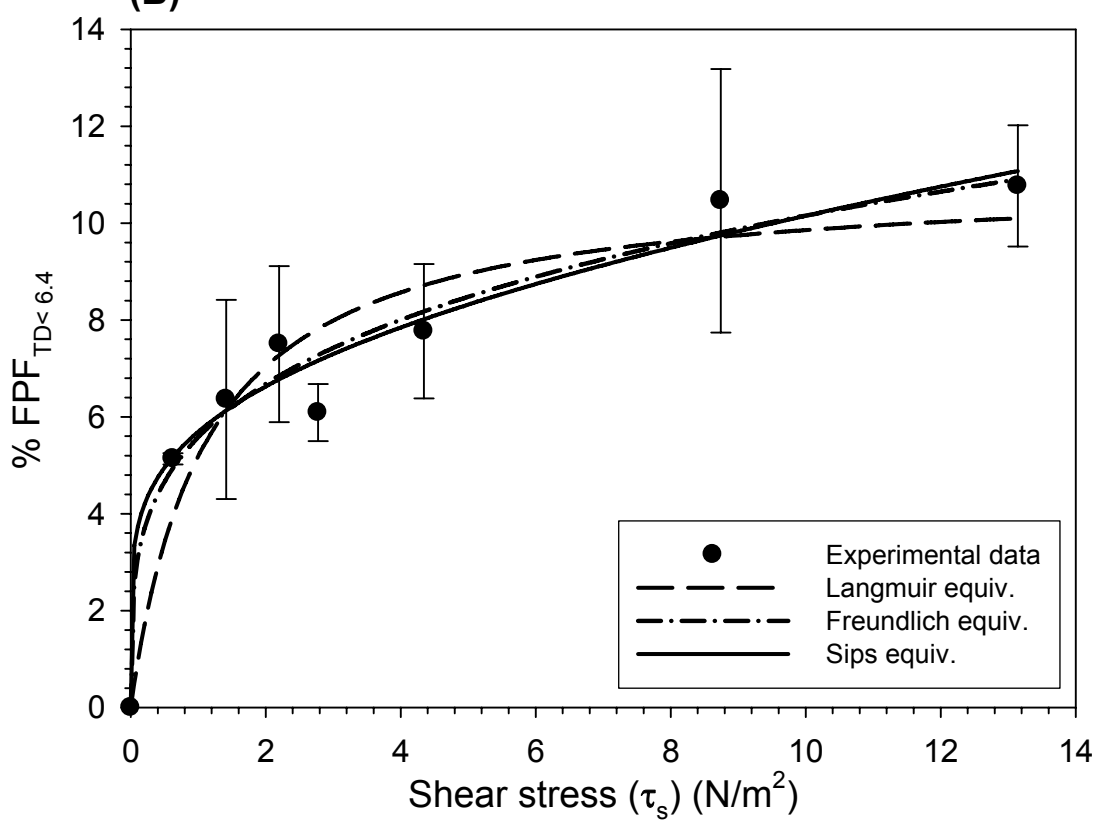


Figure 5-4. Schematic Illustration of The Different Models* of Heterogeneous Surfaces in Adsorption Theory (Adapted from [33])

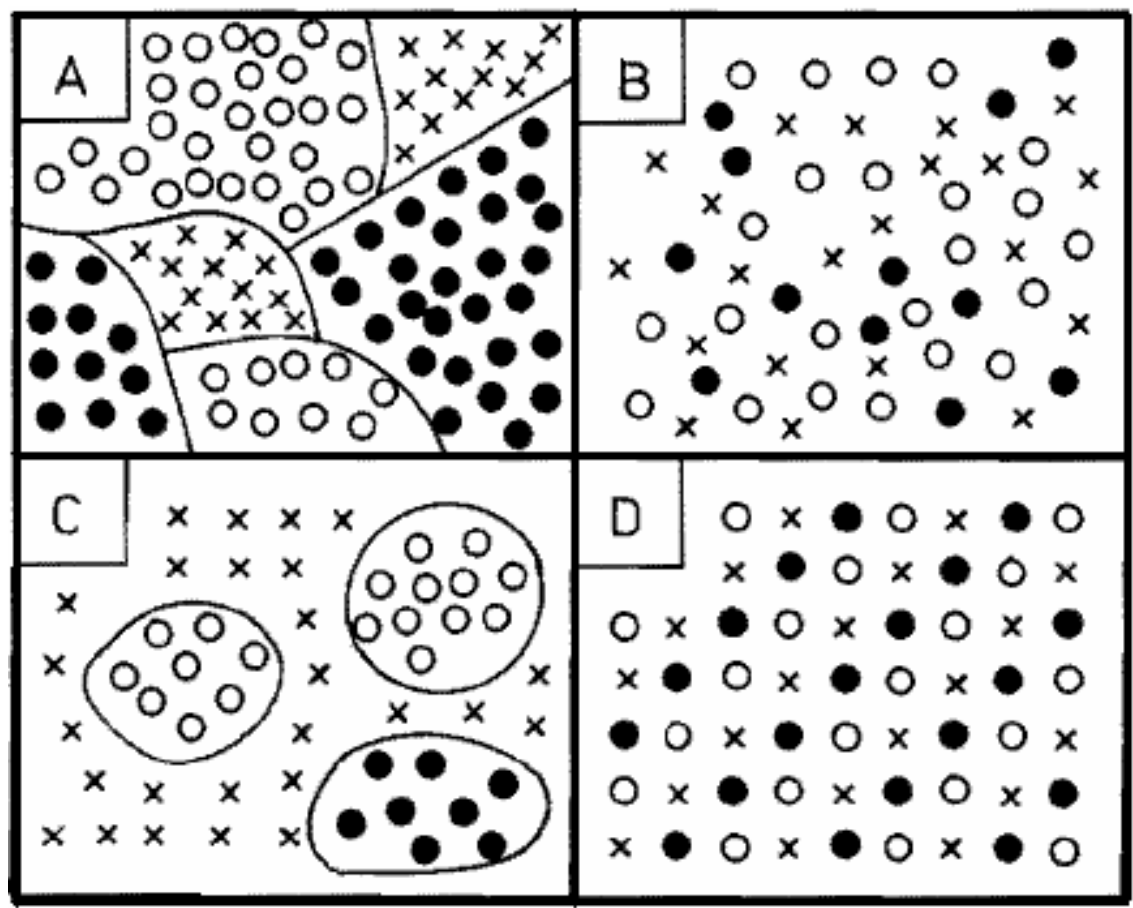

* (A) patchwise; (B) random; (C) medial; and (D) regular distributions of adsorption sites on surface 
Figure 5-5. TSLI Performance $\left(F P F_{T D}\right)$ and Shear Stress $\left(\tau_{s}\right)$ Correlation:* (A) Performance Predicted by PADE Non-linear Regression; (B) Performance Predicted by PADE Linear Regression.

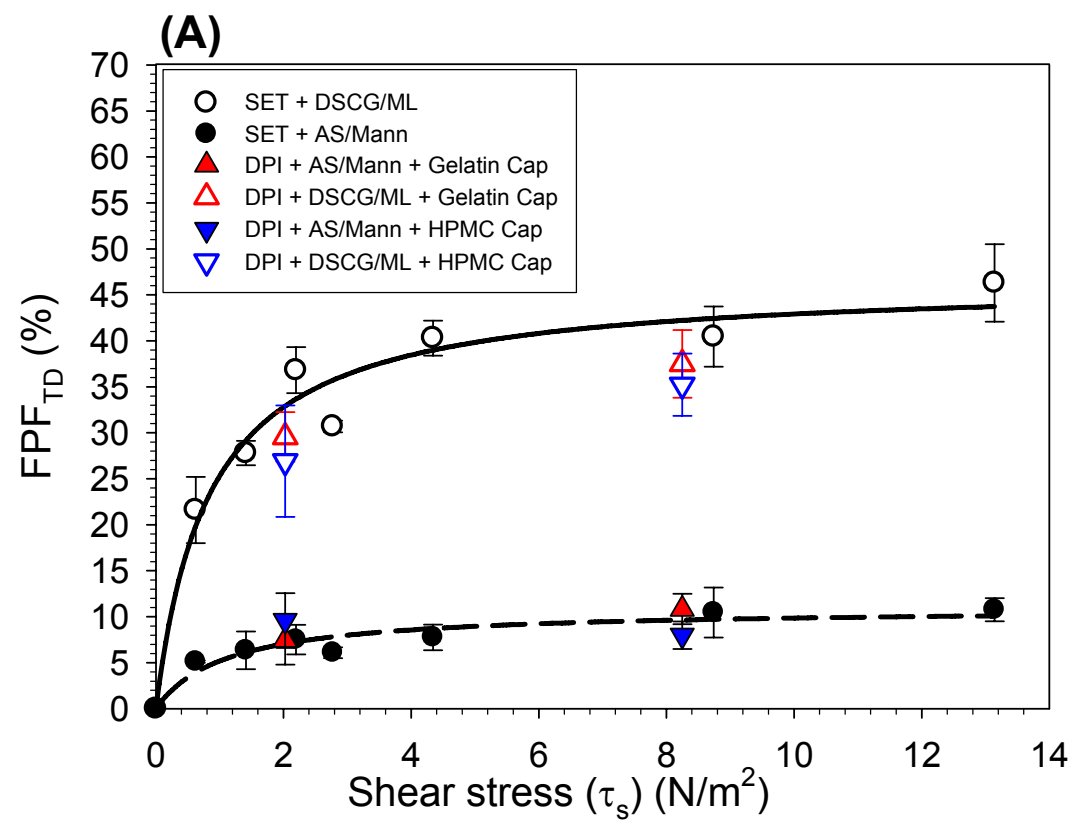

(B)

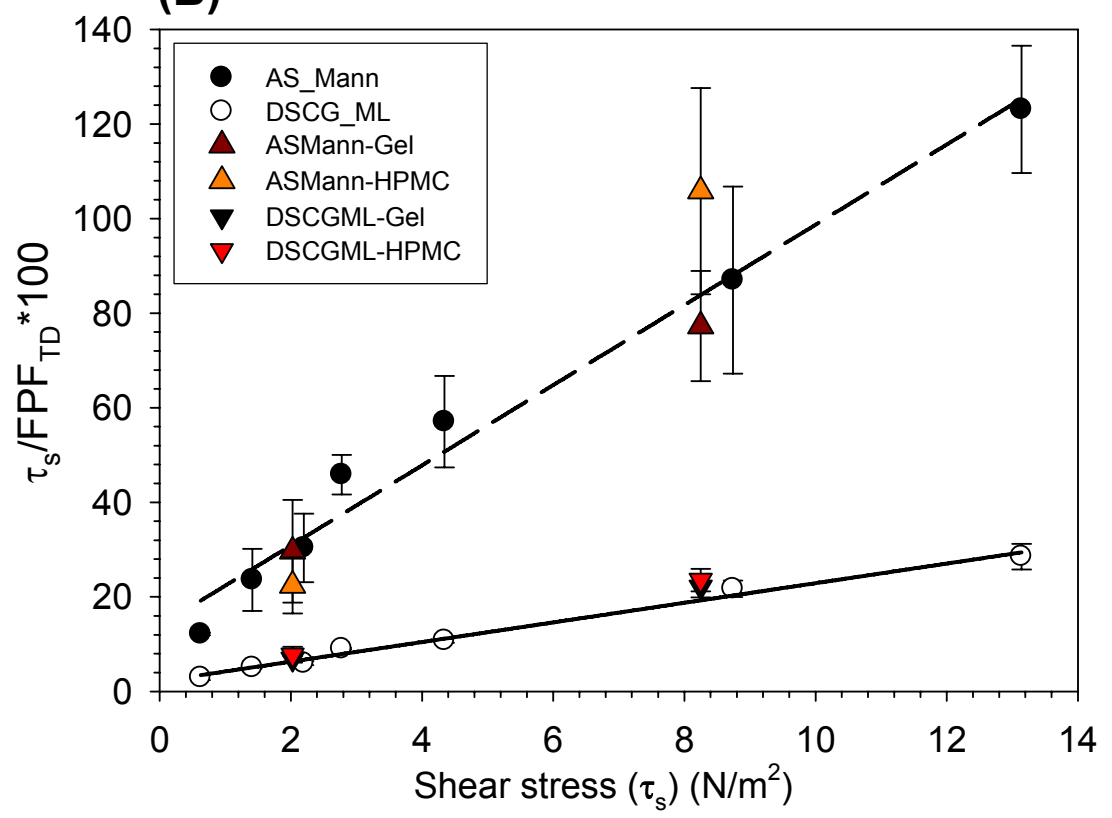

* A comparison of performance using SETs vs. DPIs (Rotahaler ${ }^{\circledR}$ and Aerolizer $\left.{ }^{\circledR}\right)$. The nonlinear regression curves were based on the data obtained from performance studies using SETs. 
Figure 5-6. The Influence of Formulation Performance upon Storage: (A) DSCG-ML780, Nonlinear Regression; (B) DSCG-ML780, Linear Regression; (C) FP-Treh, Nonlinear Regression; (D) FP-Treh, Linear Regression.
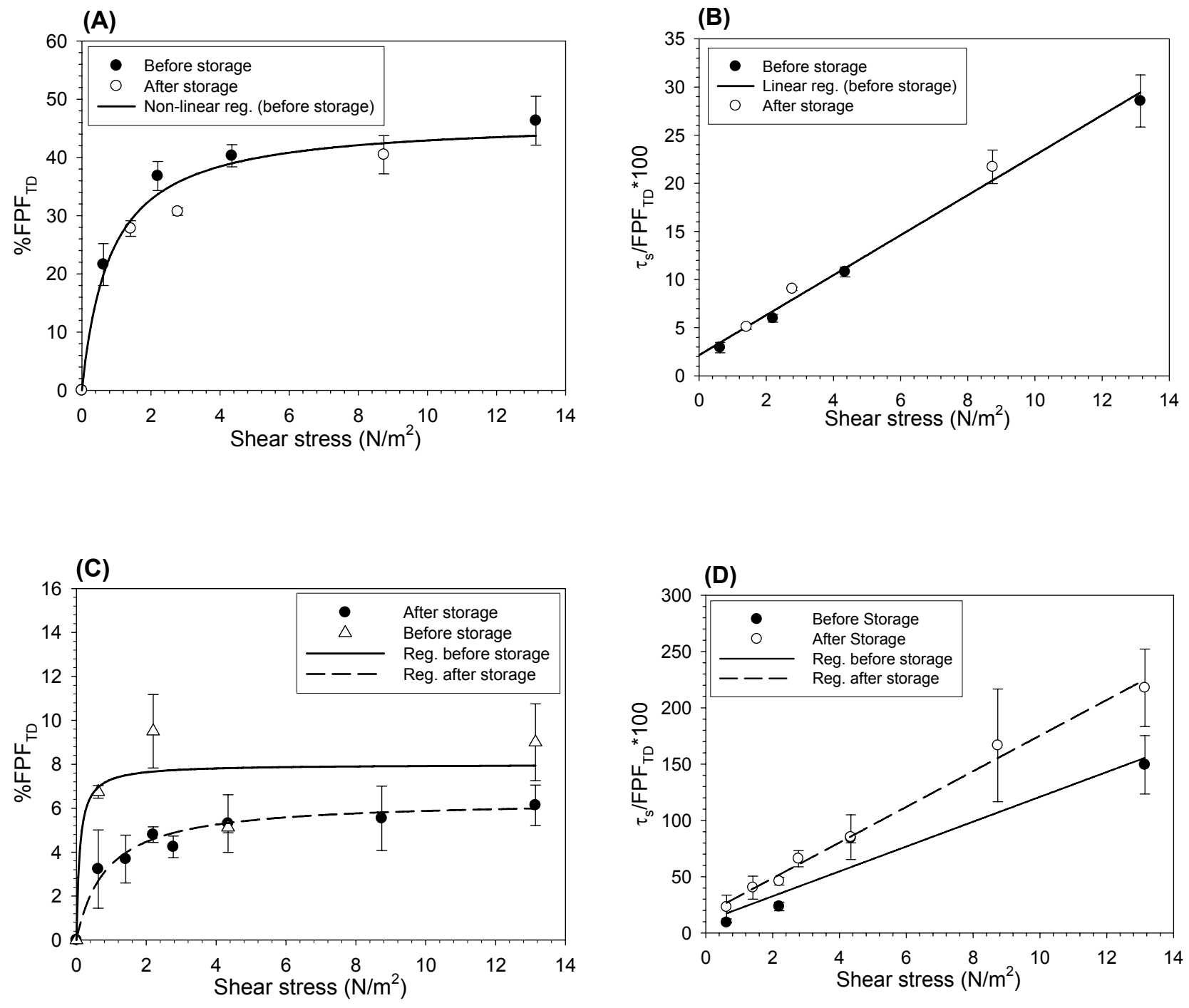


\section{References}

1. I. Langmuir, J Am Chem Soc, 38, 2221-2295 (1916).

2. I. Langmuir, J Am Chem Soc, 40, 1361-1403 (1918).

3. A. Martin, P. Bustamante and A.H.C. Chun, in: Physical pharmacy: physical chemical principles in the pharmaceutical sciences, pp. 379-392. Lea \& Febiger, Malvern, PA, (1993).

4. A.W. Adamson and A.P. Gast, Physical chemistry of Surfaces. John Wiley \& Sons, Inc., NY (1997).

5. J.N. Israelachvili, Intermolecular and Surface Forces. Academic Press, London, UK (1992).

6. W.H. Finlay, The Mechanics of Inhaled Pharmaceutical Aerosols: An Introduction. Academic Press, London, UK (2001).

7. M.D. Louey, M. VanOort and A.J. Hickey, J. Aerosol Sci., 37, 1520-1533 (2006).

8. Y.S. Ho, Carbon, 42, 2113-2130 (2004).

9. D.D. Do, Adsorption analysis: equilibria and kinetics. Imperial College Press, London, UK (1998).

10. Z. Xu, H.M. Mansour, T. Mulder, R. McLean, J. Langridge and A.J. Hickey, J Pharm Sci, (2010 in press).

11. Z. Xu, H.M. Mansour, T. Mulder, R. McLean, J. Langridge and A.J. Hickey, J Pharm Sci, (2010 in press).

12. H.M. Mansour, Z. Xu and A.J. Hickey, J Pharm Sci, (2010 in press).

13. Z. Xu, H.M. Mansour, T. Mulder, R. McLean, J. Langridge and A.J. Hickey, J Pharm Sci, (2010 in press).

14. W. Rudzinski and D.H. Everett, Adsorption of gases on heterogeneous surfaces. Academic Press, San Diego (1992).

15. J.R. Coury and M.L. Aguiar, Powder Tech., 85, 37-43 (1995).

16. C.H. Giles, D. Smith and A. Huitson, J Colloid Interface Sci, 47, 755-765 (1974).

17. C.H. Giles, D. Smith and A. Huitson, J Colloid Interface Sci, 47, 766-778 (1974).

18. H.M.F. Freundlich, J Phys Chem, 57, 385-470 (1906). 
19. R. Sips, J Chem Phys, 16, 490-495 (1948).

20. J. Toth, Adv Colloid Interface Sci, 55, 1-239 (1995).

21. A.W. Adamson and A.P. Gast, Physical chemistry of Surfaces. John Wiley \& Sons, New York (1997).

22. S. Brunauer, P.H. Emmett and E. Teller, J Am Chem Soc, 60, 309-319 (1938).

23. T.L. Hill, J Chem Phys, 14, 263-267 (1946).

24. A.R. Clark and A.M. Hollingsworth, J Aerosol Med, 6, 99-110 (1993).

25. C.A. Dunbar, A.J. Hickey and H. P., Kona, 16, 7-45 (1998).

26. M.S. Coates, H.K. Chan, D.F. Fletcher and J.A. Raper, Pharm Res, 22, 1445-53 (2005).

27. M.S. Coates, H.K. Chan, D.F. Fletcher and H. Chiou, Pharm Res, 24, 1450-6 (2007).

28. M.A. Braun, R. Oschmann and P.C. Schmidt, Int J Pharm, 135, 53-62 (1996).

29. L. Borgstrom, L. Asking and P. Lipniunas, J Aerosol Med, 18, 304-10 (2005).

30. J.S. Cox, G.D. Woodard and W.C. McCrone, J Pharm Sci, 60, 1458-65 (1971).

31. L.R. Chen, V.G. Young, Jr., D. Lechuga-Ballesteros and D.J. Grant, J Pharm Sci, 88, 1191-200 (1999).

32. M.D. Jones, J.C. Hooton, M.L. Dawson, A.R. Ferrie and R. Price, Int J Pharm, 313, 87-98 (2006).

33. M. Jaroniec and R. Madey, Physical Adsorption on Heterogeneous Solids. Elsevier Science Publishers, New York (1988). 


\section{CHAPTER 6 SURFACE MODIFICATION FOR LACTOSE}

\subsection{Introduction}

Instead of searching for alternative carriers, surface modification (e.g. surface coating, etching, smoothing) is another strategy that manipulating the interparticulate forces and the corresponding performance optimization. The particle core is not critical to functionality other than its size and size distribution when the particle surfaces are applied with thin layer of coated material, often called force control agents. A controlled coating layer can effectively mask the particle surface heterogeneity and enable optimization of interfacial properties (adhesion and friction) of drug and carrier particles. The surface coating in dry powder formulation can change the physicochemical properties such as stability, hygroscopicity [1], flowability and dispersibility [2]. The coating of carrier particles with low surface energy material has been reported to increase the aerosolization efficiency of DPI formulations.[3-8] Before delving into the surface coating and performance studies, some background information including the proposed ternary blending mechanisms, the nature of particle surfaces, and coating techniques for dry powder aerosols, will be discussed first.

\subsubsection{Proposed Ternary Blending Mechanisms}


Jones et al [9] reviewed the influence of various ternary blending of lactose or other fines of carrier-based dry powder formulations. It is known that the addition of fines (typically volume median diameter of $<10 \mu \mathrm{m}$ for lactose) of many different materials improves formulation performance, as shown by increased fine particle fraction (FPF), whereas the removal of intrinsic fines from a lactose carrier has been shown to decrease formulation performance. Furthermore, there exists an optimal concentration of fines for maximum FPF.[10] The influence of fines on the formulation performance is consistent even though different formulation and methodologies were used.[9, 11] However, the mechanism by which fines improve formulation performance differs. Two debatable mechanisms of ternary blend increasing FPF have been proposed regarding the nature of lactose-drug interactions influencing the efficiency of particle separation and, therefore, drug aerosolization. Each mechanism has its supporting empirical evidence.

- Active sites: which give rise to greater binding of fine particles at carrier surfaces;

- Agglomerates: of lactose fines and drug at the surface of carrier particles that are dispersed at low shear.

\subsubsection{Proposed “Active Site” Mechanism (Figure 6-1)}

It was proposed that high-energy active sites exist on the coarse carrier particles [12]. The addition of ternary fine particles preferentially binds to these highly adhesive sites, forcing the drug to the weaker binding site, thus drug particles are more easily liberated from the surface of the carrier particles after actuation. The surface area distribution function for heterogeneity in surface energy can be probed by $\mathrm{N}_{2}$ adsorption isotherm 
using energy density function theory.[13] The presence of high energy sites on the carrier surface can also be detected by atomic force microscopy (AFM) spatial adhesion map and phase diagram.[14-17] If this hypothesis is true, the active sites should be saturable, and the order of mixing of drug, carrier, and fine will result in performance difference, according to ordered mixing of cohesive/adhesive interactive physical mixture.[84] Young et al [15] studied the influence of dose on the performance of dry powder formulation and found that no statistically significant difference in fine particle dose (FPD) at low dose range until the occupancy of "active sites" are saturated. Zeng et al [18] found that the blending of coarse lactose/fines before adding drug gave superior FPD to the blending of drug/coarse lactose first. The FPD of lactose produced gave opposite result. Furthermore, lactose fines enough for monolayer coverage gave good FPD, whereas too much fines resulted in poor FPD. These experimental evidences support the "active site" mechanism. However, the formulation performance was observed to be independent of the blending order in some other systems.[19, 20] The blending time could arguably be an influencing factor since the interactive physical mixing involves statistical and time-dependent particle redistribution. Zeng et al [18] suggested that the effect of blending order is also dependent on the particle size of the fines, the device and airflow rate applied. It was also found by AFM pull-off force measurement that active sites still existed and the adhesion forces on the carrier surface increased after addition of ternary component.[21] Moreover, Louey et al [20] found evidence that the addition of albuterol sulfate particles can in fact displace intrinsic fine lactose particles from carrier, thus casting doubt on the "active site" mechanism. It was speculated that competitive and 
multilayer adhesion may produce equilibrium mixtures where drugs could interact with both carriers and surface fines.

\subsubsection{Proposed "Agglomerates" Mechanism (Figure 6-2)}

The inconsistencies in evidence for the previous mechanism led to the introduction of an alternative "agglomerates" mechanism. It was proposed that during interactive mixing, drug particles were distributed between the surface of carrier and fines, forming weak agglomerates of drug and fines that could be dispersed easily. The direct experimental observation $[19,20]$ of this mechanism was the drug and fine agglomerates by scanning electron microscopy.[22, 23] Louey et al [20] suggested that multilayer and multiplet "agglomerates" had greater detachment mass and was more easily librated from the carrier. Smaller drug-fine agglomerates could also contribute to the drug deposition without being separated. Islam et al [10] observed that an optimum drug and lactose count ratio of 1:1 exists when studying the salmeterol xinafoate blends. This can be explained in terms of agglomerates of drug and lactose fines weakening the cohesive interactions between drug particles. An investigation [24] of the mechanism by AFM cohesive-adhesive balance $(\mathrm{CAB})$ approach suggested that increasing relative drug-fines adhesion gave rise to larger drug-fines agglomerates which would experience greater aerodynamic forces and kinetic energies for deaggregation, thus increase performance. However, this observation is limited to the drug being more adhesive than cohesive. The situation when the drug being more cohesive than adhesive is still unclear.[9] Similarly, Cline et al [25] examined the surface energy interaction of several drugs and carriers by inverse gas chromatography (IGC), and correlated it with formulation performance. These 
researchers also found the increasing of surface energy between drug and carrier resulted in greater fine particle fraction of drug. No substantial difference was observed between surface free energy values of different lactose batches even though the amount of intrinsic fines was different.

Up to now, there's no definitive conclusion regarding which of the proposed mechanisms prevail, owing to the myriad of formulation parameters (such as different drugs, carriers, different size, size distribution, morphology of drug and carrier particles) and diverse methodologies (such as different techniques for adhesive force measurement, different devices) employed by different research groups. The evidence in support of either mechanism is limited and sometimes contradictory. For example, Podczeck [26] used a centrifuge technique to assess the adhesion between salmeterol xinafoate and lactose carriers with or without lactose fines. The adhesion increased with the addition of fines. However, Lord and Staniforth [27] used a different centrifuge technique to evaluate the adhesion forces between an albuterol sulfate $(0.8 \% \mathrm{w} / \mathrm{w})$ and lactose formulation. They found that inclusion of lactose fines $(\mathrm{VMD}=12.6 \mu \mathrm{m})$ resulted in decreased adhesion between drug and carrier. These findings indicated that the ternary blending mechanisms could be formulation dependent. It also indicated that the phenomenology based on the surface analytical techniques may not directly account for the aerosolization performance. Moreover, to elucidate the mechanisms, it is necessary to develop a theoretical description of the powder deaggregation process including the fundamental forces of interaction that occur during the aerosolization of ternary inclusion formulation, as well as a standardized methodology to employ it. 


\subsubsection{The Nature of Particle Surfaces}

It is generally known that the surfaces of dry powder aerosol formulations are heterogeneous. However, describing the surface heterogeneity by dividing carrier surface into areas of strong and weak adhesion (active and non-active sites, shown in majority of literatures that support the "active" site mechanism) may be too simple. A few other studies proposed that the filling of fines in the carrier crevices or pores may prevent the drugs from being trapped by mechanical interlocking.[28, 29] More recently, studies using AFM colloid probe techniques may shed light on the nature of particle surfaces. A rule of thumb from these studies indicated that both heterogeneous asperity (geometric variations in the contact zone) and heterogeneous surface energy would cause logarithmic normal distribution of the adhesive forces.[30, 31]

In principle, the heterogeneity manifests on the particle surfaces should be similar as that described in the surface adsorption or surface chemistry since the fundamental forces and surface energies are described and characterized similarly.[32, 33] In surface adsorption theory, heterogeneity can be roughly classified from geometric and energetic perspectives (see Figure 5-4). The former includes particle size, size distribution (including the presence of a ternary component), morphology, surface asperities, meso-/micropores, and particle deformation, and the latter, the energy density difference caused by surface imperfection such as amorphous content, polymorphic state, or surface impurities. Even perfectly crystalline materials have different energy distributions due to their molecular orientation and crystal habit. The form of distribution varies with different dry powder material. They will influence the interparticulate forces, heat capacity, 
mechanical stress, etc. The practical approach to describing energy heterogeneity (such as that employs in adsorption theory or protein binding) is to average local thermodynamic quantities and treat them statistically (i.e. distribution function).[33] Several distribution functions have been used in the literature, such as uniform distribution, normal distribution, log-normal distribution, exponential distribution and Rayleigh distribution. In addition, the surface heterogeneity feature is a characteristic of mutual interaction between particles rather than an independent particle.

\subsubsection{Surface Coating Techniques}

Surface coating has been widely used in the pharmaceutical processing. An ideal surface coating layer will provide solely surface properties and the core material become less critical. Several different techniques have been employed for the preparation of surface modified or coated particles (either drug or carriers) for dry powder aerosol formulations. They were aimed at several purposes such as manipulation of interparticulate forces (or surface energy) to improve powder flowability and dispersibility, prevent moisture ingress and hygroscopic growth, and reduce batch to batch variability. They can be roughly divided into dry and wet coating methods.

The dry methods is virtually a mixing process that relies on mechanical (shear, impact, etc) forces that bring smaller guest particles (nano- to micro-size range) into contact with larger host particles.[2] To meet the needs of different coating requirement, the mixing process can be further classified based on the energy input such as 
conventional V-shape blending, intensive elliptical-rotor-type mixing [8, 34], and high energy machanofusion.[35]

The wet methods are more flexible and they include conventional techniques and a few technological advances. The conventional techniques involve suspensions and emulsions where particles are coated and stabilized in a solution. There are some good examples such as adsorption coacervation [36-38], fluidized bed coating (Wurster processor) [39], and spray drying [40]. A good dispersion of particles prior to coating is needed for homogeneous distribution of coating layer. Particles suspended in nonpolarized and non-conducting media (e.g. liquid nitrogen) may disperse better than in air due to the reduced Hamaker constant.[41] Recently, A new technique was introduced by diffusion or deposition of sublimed gas vapor (e.g. L-leucine at $\sim 145{ }^{\circ} \mathrm{C}$ ) in a laminar aerosol flow reactor.[42, 43] This gas phase coating allows in situ formation and surface coating of micro- to nano-sized albuterol sulfate particles.

The selection of proper coating method depends on the host particle characteristics, the availability of coating equipment, and the desired coating layer. A discrete coating layer without disrupting the host particles is desired to provide expected surface properties. Thin layer coating is desired to reduce guest material mass and avoid appreciable particle size increase. Among various coating techniques, the adsorption coacervation and mechanofusion methods may be most suitable for current studies. The former is a method of adsorption of long chain fatty acids with different chain length from non-aqueous solution to the host particles. Host micronized particles such as disodium fluorescein [36, 38, 44] and disodium cromoglycate [37] were coated with 
lauric or capric acid to prevent hygroscopic growth and facilitate powder flow properties. The advantages of this method are precision coating of fatty acid in nanometer thickness, less energy input and heat generation, and easy operation. However, Non-aqueous solvent used in this method may change the properties of host particle discrete of the coating. The latter method relies on intensive energy input to mechanically fuse a nano-layer of force control agents (FCA) onto the host particles.[35] This method is aimed at modifying surface energy and particle shape of lactose carrier.[5, 35] Using this approach, several force control agents such as L-leucine, lecithin, and magnesium stearate were coated on the surfaces of lactose carrier [4, 45] or drug particles [35]. Due to the specific equipment required for mechanofusing, this method was considered as an alternative. Instead, the adsorption-coacervation method was chosen.

The purpose of this study was to apply a thin layer of fatty acid onto the hydrophilic surfaces of lactose monohydrate carrier particles. The introduction of minimum amount of fatty acid is proposed not to enter the lung. It is hypothesized that the carrier surface treatment by fatty acid surface coating can reduce the surface heterogeneity and improve the aerosolization efficiency of dry powder aerosol formulation.

\subsection{Material and Methods}

\subsubsection{Material}


Two lactose monohydrate (Respitose ${ }^{\mathrm{TM}}$ ) designated as sieved (SV) and milled (ML) were selected from previous studies. The physicochemical properties of these two lactose monohydrate batches were reported previously. Briefly, SV powder had more smooth surface textures and regular shape, lower molecular disorder and higher crystallinity, much less surface fines than ML powder. A broader span was observed in ML $(\sim 3.1)$ than SV ( 1.1) particles, although the volume median diameters were comparable $(55 \sim 60 \mu \mathrm{m})$. The specific surface area of ML $\left(0.89 \mathrm{~m}^{2} / \mathrm{g}\right)$ was about three times that of SV $\left(0.30 \mathrm{~m}^{2} / \mathrm{g}\right)$ particles. Two micronized drugs, albuterol sulfate (AS) and fluticasone propionate (FP) were prepared previously and used immediately after passing a $125 \mu \mathrm{m}$ sieve. The interactive physical mixtures were prepared at $2 \% \mathrm{w} / \mathrm{w}$ concentration as was described previously.

The selection of fatty acids was based on: 1) Safety issue. FDA has stringent draft guidance for DPI formulation. The excipient/carrier and non-active ingredient are expected to be swallowed instead of inhaled. The introduction of minimum amount of fatty acid is proposed not to enter the lung. The naturally occurring fatty acids with even number of carbon atoms are often used in food science and human nutrition and generally regarded as safe. 2) Physicochemical consideration. As a starting point, only saturated fatty acids will be selected. Fatty acid as amphiphile is expected to adsorb onto the hydrophilic lactose monohydrate surface. Vertically orientated fatty acid occupies a surface area of $\sim 20 \AA^{2}$ per molecule.[36] Four naturally occurring fatty acids including stearic acid $\left(\mathrm{C}_{18} \mathrm{H}_{36} \mathrm{O}_{2}\right.$, MW $\left.284.5 \mathrm{~g} / \mathrm{mol}\right)$, palmitic acid $\left(\mathrm{C}_{16} \mathrm{H}_{32} \mathrm{O}_{2}\right.$, MW $\left.256.4 \mathrm{~g} / \mathrm{mol}\right)$, 
myristic acid $\left(\mathrm{C}_{14} \mathrm{H}_{28} \mathrm{O}_{2}\right.$, MW $\left.228.4 \mathrm{~g} / \mathrm{mol}\right)$, lauric acid $\left(\mathrm{C}_{12} \mathrm{H}_{24} \mathrm{O}_{2}\right.$, MW $\left.200.3 \mathrm{~g} / \mathrm{mol}\right)$ were all purchased from Sigma-Aldrich Inc. (St Louis, MO).

Based on previous selected non-polar aprotic solvents that were used for adsorption studies, dichloromethane (anhydrous, $\mathrm{CH}_{2} \mathrm{Cl}_{2}$, Sigma-Aldrich Inc., St Louis, MO) and chloroform (anhydrous, $\mathrm{CHCl}_{3}$, Sigma-Aldrich Inc., St Louis, $\mathrm{MO}$ ) were selected. The surface tensions of $\mathrm{CH}_{2} \mathrm{Cl}_{2}$ and $\mathrm{CHCl}_{3}$ at $20{ }^{\circ} \mathrm{C}$ were reported to be 28.1 and $27.2 \mathrm{mN} / \mathrm{m}$, respectively.[46]

\subsubsection{Preparation of Fatty Acid Coated Lactose Monohydrate}

Adsorption-coacervation: Surface coated particles were prepared by dissolving $0,0.2$, $0.4,0.8,1.6,2.4,3.2,4.8,6.4,8.0$, and $10.0 \mathrm{mg} / \mathrm{mL}$ of fatty acid in $15 \mathrm{~mL}$ of dichloromethane. SV or ML (500 mg each) was placed in dried test tubes and the fatty acid solution was added. The test tubes were sealed by Teflon-wrapped stopcocks to avoid any leakage. SV or ML particles were suspended in the fatty acid solution by swirling at room temperature $\left(23.5 \pm 1{ }^{\circ} \mathrm{C}\right)$. After $6 \mathrm{~h}, 3 \mathrm{~mL}$ of chloroform was added to the suspension to promote coacervation. Seventy-two hours after initial preparation, the coated powders were flash dried using a Buchner flask and Whatman 50 hardened filter paper at ambient condition $\left(23.5 \pm 1{ }^{\circ} \mathrm{C}, \mathrm{RH} \%\right.$ : $\left.25 \sim 44\right)$. The collected powders were dried in a vacuum oven at room temperature overnight to remove trace amount of solvent. The powders were then stored in desiccator at ambient temperature. 
Quantification of Coated Fatty Acid: The coated SV or ML samples (250 mg) were placed in $20 \mathrm{~mL}$ test tubes. Dichloromethane $(5 \mathrm{~mL})$ and deionized water $(10 \mathrm{~mL}$, Barnstead Nanopure, 18.0 mega-ohm) were added to each test tube. The mixtures were shaken vigorously and the bottom layer $\left(\mathrm{CH}_{2} \mathrm{Cl}_{2}\right)$ was collected in scintillation vials and dried over anhydrous sodium sulfate (Aldrich-Sigma). An equal volume (4 mL) of collected dichloromethane, together with a standard fatty acid (palmitic acid for analyzing stearic acid, myristic acid for analyzing palmitic acid, lauric acid for analyzing palmitic acid, and palmitic acid for analyzing lauric acid) solution $(5 \mathrm{mg} / \mathrm{mL})$ in dichloromethane $(1 \mathrm{~mL})$. The solvent was evaporated. The resulting fatty acids (with internal fatty acid standard) were derivatized by adding $14 \%$ borotrifluoride in methanol (Sigma-Aldrich, Inc., St Louis, MO). The solutions were heated in boiling water for 2 min for complete derivatization. After cooling to room temperature, the methyl ester was extracted by adding petroleum ether $\left(10 \mathrm{~mL}, 40-60{ }^{\circ} \mathrm{C}\right.$, Acro, $\left.\mathrm{NJ}\right)$ and deionized water $(15 \mathrm{~mL})$. The top layer (petroleum ether) was extracted and dried once more over anhydrous sodium sulfate. The prepared methyl ester solution was added into cuvette for Gas Chromatography (GC) analysis.

The amount of fatty acid associated with lactose monohydrate was determined by GC analysis (Shimadzu GC-2014, Maryland, USA). The GC column used for fatty acid quantification was a capillary column $30 \times 0.32$ with $1 \mu \mathrm{m}$ film thickness (Alltech Heliflex AT-1000, Deerfield, IL). The injection port temperature, the oven/column temperature, and the detection temperature were set at 200,180 , and $250{ }^{\circ} \mathrm{C}$, respectively. The detection temperature for lauric acid was set to $160{ }^{\circ} \mathrm{C}$. A flame ionization detector 
was used with a mixture of hydrogen (IND $176 \mathrm{CF}$ ) and air (zero grade, 300CF). Helium (research grade 6.0) was used as the convey gas flowing at $27.8 \mathrm{~mL} / \mathrm{min}$. The retention time for derivatized fatty acid standard was obtained in Figure 6-2. The amount adsorbed and the equilibrium concentration was correlated to obtain the adsorption association isotherm.

According to the adsorption curve obtained, two equilibrium concentrations (designated as low and high) were chosen. The adsorption-coacervation was repeated at these two fatty acid equilibrium concentrations but at larger scale. SV or ML (10 g) was used and the fatty acid solution was drawn to scale. The original (+SVcontrol and + MLcontrol $)$ and solvent treated $(0 \mathrm{mg} / \mathrm{mL}$ fatty acid) (-SVcontrol and -Mlcontrol) lactose monohydrate were used as control. The prepared SV or ML was stored in desiccicator before blending.

\subsubsection{Blending}

The drugs were micronized albuterol sulfate (AS) and fluticasone propionate (FP) (see

Chapter 3). Four interactive physical mixtures (2 drugs: AS and FP; 2 carriers: original SV and ML) were selected, twelve new interactive physical mixtures (2 drugs; 4 stearic acid coated carriers and 2 solvent treated control) were prepared using the same blending method described in previous chapters. The eight carriers included four batches each of sieved (SV) and milled (ML) lactose monohydrate. They were symbolized as original control (+SVcontrol and +MLcontrol), solvent treated control (-SVcontrol and 
-MLcontrol), low equilibrium concentration stearic acid coating (SVlow and MLlow), and high equilibrium concentration stearic acid coating (SVhigh and MLhigh). The blending homogeneity for all the formulations were tested and a coefficient of variation smaller than $5.0 \%$ was considered homogeneous.

\subsubsection{Physicochemical Characterization}

\subsubsection{Bulk/tapped density (powder flow)}

The bulk and tapped densities were determined using a modified method (reduced volume due to limited amount of powder) before and after fatty acid coating. Approximately 10 $\mathrm{mL}$ of accurately weighed powder was employed to conduct each measurement by pouring into a glass syringe. The volume of powder was determined by measuring the cross-sectional area and the height using a caliper. The tapping was achieved by dropping the syringe vertically for $>200$ times at a height of $0.5 \mathrm{~m}$. The height of powder was determined when no further decrease of height was observed. The experiment was repeated $(n=3)$ and average bulk and tapped densities were calculated. The Carr's compressibility index (CCI) was determined by using Eq. 6-1.[47] The CCI is a parameter for quantifying the powder flow. As powder flow improves, the bulk density approaches the tap density.[47] Smaller CCI corresponds to improved powder flow.

$$
C C I=\frac{\text { Tap density }- \text { Bulk density }}{\text { Tap density }} \times 100 \% \quad \text { (Eq. 6-1) }
$$




\subsubsection{Thermal properties (DSC)}

The thermotropic phase transitions of SV and ML before and after coating, and interactive physical mixtures were examined by DSC (Perkin Elmer DSC 6 Workstation, Norwalk, CT). Non-hermetically sealed aluminum pans were used. The heating rate was set at $5{ }^{\circ} \mathrm{C} / \mathrm{min}$ from $20-250{ }^{\circ} \mathrm{C}$. Thermograms were processed and analyzed using the accompanying software (Pyris Thermal Analysis Instrument Control and Data Analysis Software, v.3.01).

\section{$\underline{\text { 6.2.5 In Vitro Aerosolization Performance Assessment }}$}

The aerosolization performance of twelve new formulations after carrier coating with stearic acid and blending with AS or FP was assessed using twin-stage liquid impinger (TSLI). The setup and actuation for TSLI was described previously ( $25 \mathrm{mg}, 10 \mathrm{~s}$ at 60 $\mathrm{L} /$ min for each actuation). The PADE non-linear and linear regression analyses were used to correlate the aerosol performance with shear stress. The statistical analysis was carried out using SigmaPlot software. (SigmaPlot 2004 for Windows Ver 9.01, Systat Software, Inc., CA).

\subsection{Result and Discussion}

\subsubsection{The Theoretical Estimation for Monolayer Surface Coating}


The lactose surface area of SV and ML powders were measured using $\mathrm{N}_{2}$ adsorption and Brunauer Emmett, and Teller equation to be $0.30 \mathrm{~m}^{2} / \mathrm{g}$ and $0.89 \mathrm{~m}^{2} / \mathrm{g}$, respectively. Vertically orientated fatty acids occupy a surface area of $\sim 20 \AA^{2}$ regardless of chain length.[36] Based on the assumption that the each fatty acid would occupy the same vertical surface area for a monolayer surface coating, the mass of fatty acid associated with a unit mass of lactose monohydrate can be calculated according to the Eq. 6-2:[36] where $S S A$ is the specific surface area of lactose; $S_{a}$ is the surface area of standing fatty acid ( $\mathrm{m}^{2} /$ molecule); $N_{A}$ is Aveogadro's constant; $M_{W}$ is the molecular weight of the fatty acid; $C_{a d s}$ is the mass of fatty acid associated with a unit mass of lactose $(\mathrm{g} / \mathrm{g})$.

$$
C_{a d s}=\frac{S S A \cdot M_{W}}{S_{a} N_{A}} \quad \text { (Eq. 6-2) }
$$

The calculated amount of fatty acid for a monolayer surface coverage on SV and ML will be: 1) stearic acid: $0.71 \mathrm{mg} / \mathrm{g}$ and $2.1 \mathrm{mg} / \mathrm{g}$; 2) palmitic acid: $0.64 \mathrm{mg} / \mathrm{g}$ and 1.91 $\mathrm{mg} / \mathrm{g} ; 3)$ myristic acid: $0.57 \mathrm{mg} / \mathrm{g}$ and $1.70 \mathrm{mg} / \mathrm{g}$; 4) lauric acid: $0.50 \mathrm{mg} / \mathrm{g}$ and $1.50 \mathrm{mg} / \mathrm{g}$.

\subsubsection{Fatty Acid Coating of Lactose Monohydrate}

The adsorption-association isotherms for all four acids at the surface of SV and ML are shown in Figure 6-3. The GC quantification result is shown in Appendix 6-A. The result indicated that at fatty acids equilibrium concentration range between $0.2-0.8 \mathrm{mg} / \mathrm{mL}$, the amount of fatty acid coated on the surface of SV or ML was in a range of 4-250 $\mu \mathrm{g} / \mathrm{g}$, which was much less than required for a monolayer surface coating, assuming fatty acid 
stood vertically on the surface of lactose monohydrate. Linear regression of the corresponding isotherm gave excellent $\mathrm{R}^{2}$, ranging from 0.9693 to 0.9961 . This indicated that the adsorption was still in the linear range. There was a trend that the amount coated on the unit mass of ML was higher than that of SV, except for stearic acid coating. However, considering three times larger of specific surface area of ML, the amount of fatty acid coated on the ML was less than expected. The amount of coating seemed to be independent of the fatty acid chain length. This was different from an earlier study that larger amount of fatty acid coated on the disodium cromoglycate when the fatty acid chain length increased.[37] It was reported that the critical micelle concentration (CMC) for lauric acid in dichloromethane was $\sim 0.1 \mathrm{M}$, and the CMC reduced in dichloromethane and chloroform mixture due to the reduced dielectric constant of cosolvent (dielectric constant at $20{ }^{\circ} \mathrm{C}$ for dichloromethane and chloroform are 9.10 and 4.81, respectively).[36] The CMC for stearic acid in 1,2-dichloroethane (a solvent having dielectric constant 4.65 at $20{ }^{\circ} \mathrm{C}$ ) was $\sim 0.04 \mathrm{M}$. [48, 49] This indicated that the fatty acids were dissolved in dichloromethane in the concentration range studied. Reduced CMC occurred when chloroform was added, which drove the adsorption process to coacervation. Unlike the surface coating of disodium fluorescein using lauric acid [36], the step adsorption was not observed in all studied adsorption isotherm. This is conceivable since the surfaces of SV and ML were not yet saturated.

The surface coating of SV and ML using stearic acid at equilibrium concentrations of $0.4 \mathrm{mg} / \mathrm{mL}$ (SVlow and MLlow: coated mass $\sim 6 \mu \mathrm{g} / \mathrm{g}$ ) and $5 \mathrm{mg} / \mathrm{mL}$ (SVhigh and 
MLhigh: coated mass $\sim 140 \mu \mathrm{g} / \mathrm{g}$ ) were chosen for the further studies.

\subsubsection{Physicochemical Characterization}

\subsubsection{Bulk/Tap Density (CCI)}

The flow properties characterized by measuring Carr's compressibility index were shown in Table 6-1. The result indicated that all tap density values for SV were smaller than those of ML. The bulk density for SV was strongly influence by solvent treatment and coating, whereas ML was less dependent on solvent treatment and coating. When SV was treated with solvent, the flow property indicated by CCI was poorer $(0.32)$ than the original lactose (0.26). Stearic acid coating greatly ameliorate the flow properties and the CCI reduce to 0.22. Surface coating had smaller influence to the CCI of ML. But the solvent treatment resulted in the poorest flow property $(0.42)$.

\subsubsection{Thermal properties (DSC)}

DSC thermograms of -SVcontrol, -MLcontrol, SVlow, MLlow, SVhigh, MLhigh, and their corresponding AS and FP interactive physical mixtures at $5{ }^{\circ} \mathrm{C} / \mathrm{min}$ heating rate are shown in Figure 6-4. The analysis of the dehydration of the SV sample indicates that the water contents were reduced when increasing concentrations of stearic acid were used. While comparing the dehydration peak of +SVcontrol (original SV) with $-\mathrm{SV}$ control (solvent treated SV), the enthalpies are comparable. It indicated that stearic acid may participate in the dehydration on the SV lactose surface. The fusing peak was reduced as well when increasing amount of stearic acid was used, indicating the molecular 
interactions of fatty acid with anhydrous $\alpha$-lactose. It means that although very small amount of stearic acid exist on the surface of lactose monohydrate, the molecular interaction between them could be strong. The reduced dehydration and fusing peak intensity due to added stearic acid could also be observed in ML but to a less extent. But when the dehydration of - MLcontrol was compared with +MLcontrol, a significant loss of bound water was observed. Both dehydration and fusing peaks in + MLcontrol were smaller than $+\mathrm{SV}$ control. The solution treatment changed the surface molecular disorder, as the characteristic exothermic peak disappeared in both ML and SV samples.

When AS was blended with SV or ML, there was a distinct broadening of the fusing peak, indicated by the smaller onset and larger end temperature. Interestingly, when FP was blended with stearic acid coated ML or SV, there was a small peak at temperature $235{ }^{\circ} \mathrm{C}$, indicating the fusing of $\beta$-lactose. It is not clear if the stearic acid may induce the mutarotation that convert the $\alpha$-lactose to the $\beta$ form. This phenomenon was not observed in all previously studied lactose monohydrate formulations. When FP was blended with ML, the exothermic peak became visible again, indicating the disturbed surface molecules due to mechanical blending.

\subsubsection{In Vitro Aerosolization Performance Assessment (TSLI)}

The aerosolization performance results are shown in Appendix 6-B and 6-C. The performance data were correlated with SET shear stress and PADE non-linear regression and linear regression were plotted in Figure 6-5 and 6-6. In all studied systems, the solvent treatedment (-SVcontrol and -MLcontrol) resulted in negative influence on the 
aerosolization performance. This is possibly because the solvent $\left(\mathrm{CH}_{2} \mathrm{Cl}_{2} / \mathrm{CHCl}_{3}\right)$ dissolved the trace surface impurities such as protein and lipid, and exposed more surface heterogeneity and high surface energy sites than original lactose monohydrate surface. Because lactose monohydrate is a milk product, it is recrystallized with trace residual lipids (triglycerides, fatty acids, cholesterol) and protein (casein, lactoalbumin, whey), which are present on the surface dry particles. Although existed in tiny quantity, but these materials, some are surfactants, could naturally act as surface force control agents (e.g. change the surface hydrophobicity) and influence the performance efficiency. In SV-AS formulations (Figure 6-5A and 5B), the performance showed high dependency upon the amount of stearic acid coating. While the solvent treatment stripped the natural surface coating and increased surface heterogeneity, the artificial coating with stearic acid could mask surface active sites and increase performance efficiency. When SV surfaces were coated with larger amount of stearic acid (SVhigh+AS), there was a trend that the performance followed less to the Langmuir type PADE. The drug AS could be aerosolized at lower shear and the performance keep increasing at higher shear. This implies the surface coating with stearic acid may change the force balance and result in a redistribution of surface forces. More data points are necessary to explain this. Conversely, ML-AS formulation were less susceptible to the surface coating. While solvent treated control gave poor performance, the original ML and stearic acid coated ML resulted in similar performance efficiency, as is shown the overlap of PADE regression curves (Figure 6-5C and 5D). This implies that the performance efficiency is not solely dependent on the surface active sites. Because ML has many surface fines, they 
may act as the intrinsic ternary component and form drug-fine aggregates. While a stronger drug-fine interaction forces will favor larger agglomerates formation, introducing fatty acid may not necessary disrupt such agglomerates from forming. The performance will be remain the same. It should be noted that ML formulations still gave higher performance efficiency than SV formulations.

In SV-FP formulations (Figure 6-6A and 6B), the solvent treatment effectively reduced both the powder flow properties (ED in a range of $49-56 \%$ for SET-A and B) and drug aerosolization (FPF in a range of 1.8-4.7\%). However, when stearic acid was coated, the performance reached closer to the original SV-FP formulation. No further increase in performance was observed. This is probably because FP is a hydrophobic molecule $(\log P$ $=3.46[50])$. The surface coating with stearic acid may not effectively reduce particulate interactions between FP and carrier particles. In ML-FP formulation, the increase in FPF after coating occurred at low shear and maintained similar FPF at high shear. This implies such an increase in performance was not mainly because of agglomeration.

\subsection{Summary}

In this chapter, the influence of surface modification of selected lactose monohydrate was explored. The surface modification was achieved by adsorption-coacervation of naturally occurring fatty acids. Such modification was expected to increase the surface hydrophobicity and homogeneity. Four fatty acids were successfully coated at submonolayer concentration. The flow properties characterized by CCI indicated that 
solvent treatment of SV and ML result in poorer powder flow. The coating of fatty acid could effectively increase the powder flow. The thermal analysis indicated that surface coating with fatty acid in this manner or solvent treatment may result in partial loss of bound water of surface molecules. The aerosolization performance indicated that the PADE interpretation of heterogeneity by the analogous "surface occupancy" approach described by adsorption theory accounted for both proposed "active" site and "agglomeration" and without favoring either mechanism. By using a similar expression for aerosol performance which was limited only by the data available at various shear stresses, from which the hyperbolic curve was derived, it is conceivable that an analogous mechanistic approach to the evaluation of particle-particle (drug-drug, drug-excipient fines), particle-surface (drug-excipient carrier, excipient fines-excipient carrier) interactions might be achieved. 
Figure 6-1. Proposed deaggregation mechanisms of ternary blends

1) "Active" site mechanism

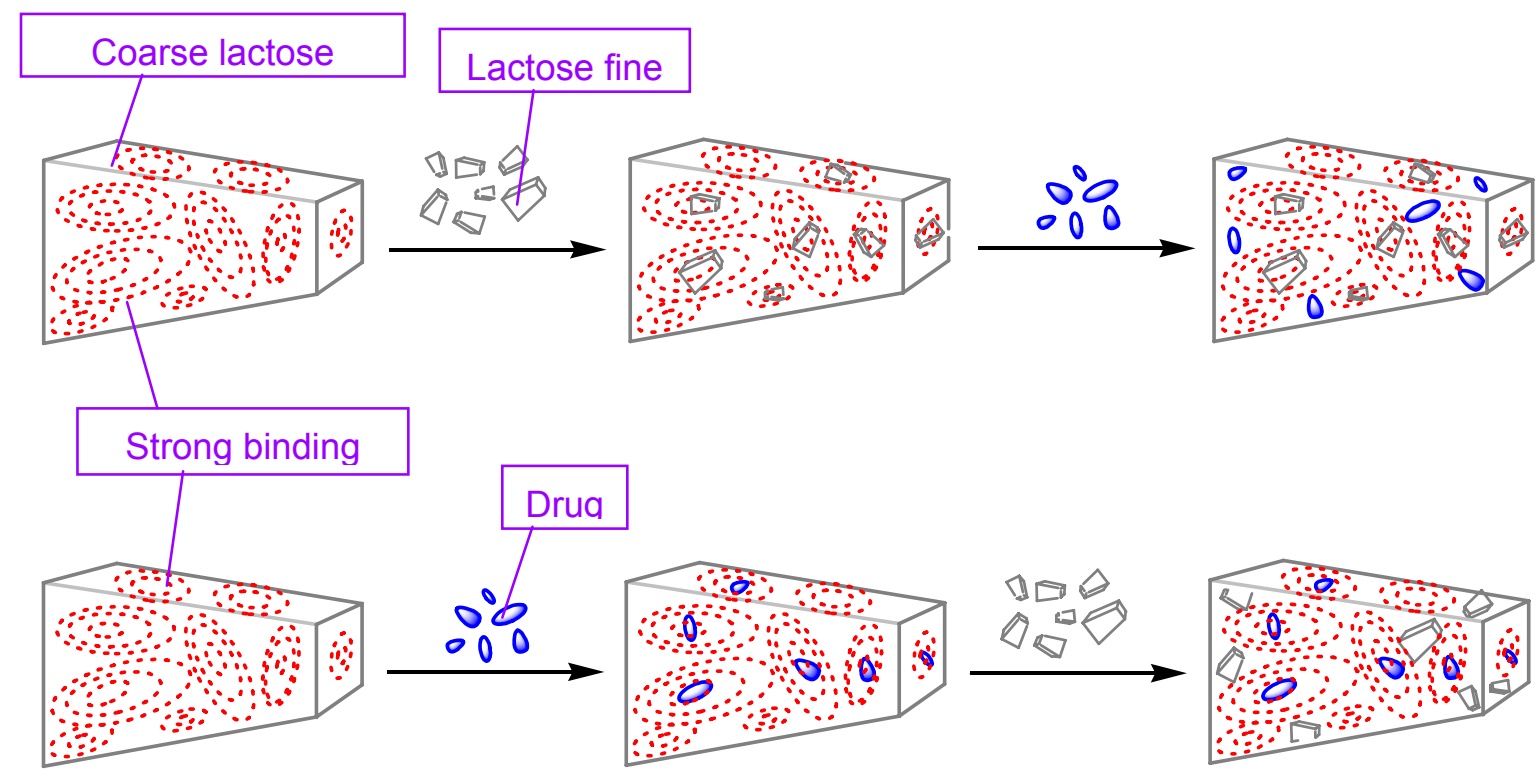

2) Deagglomeration mechanism
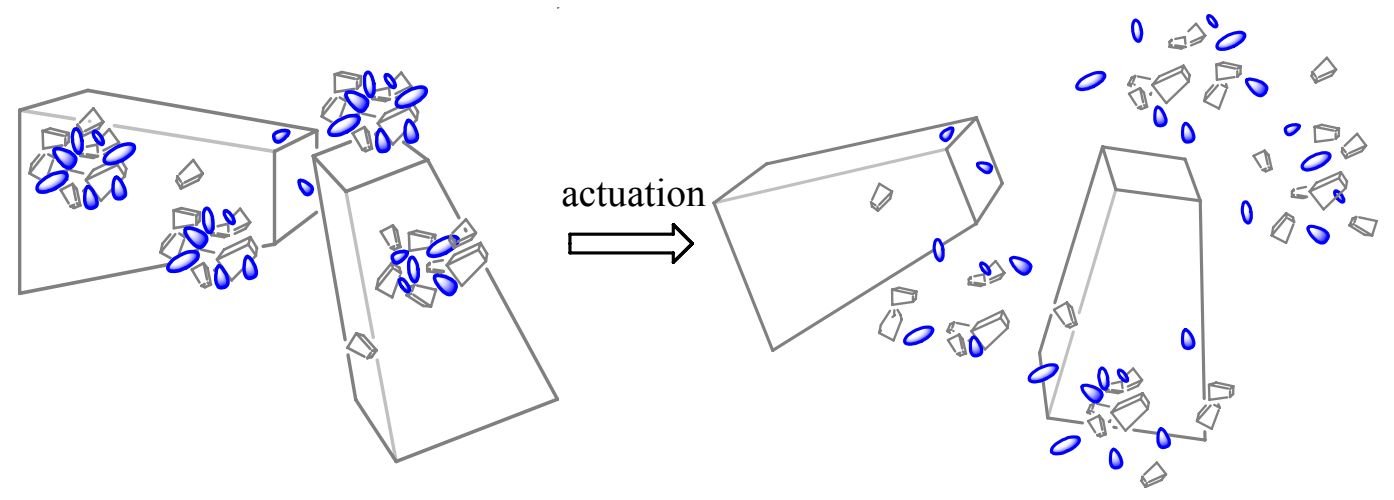
Figure 6-2. Representative Gas Chromatography Standard Peaks and Retention Time.

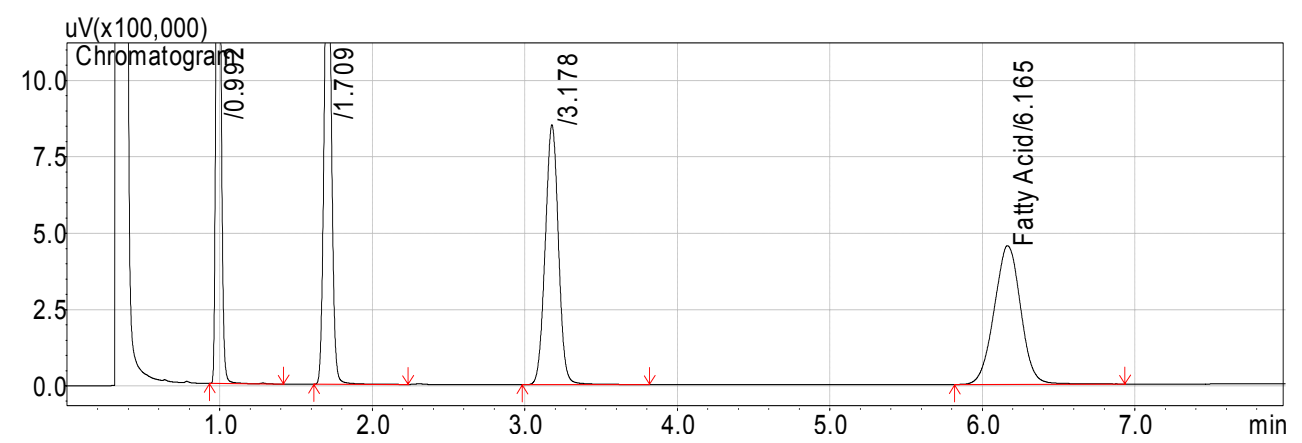

Gas Chromatography Peak Retention Time Based on Fatty Acid Chain Length.

\begin{tabular}{|c|c|c|c|}
\hline $\begin{array}{l}\text { Derivatized } \\
\text { Fatty Acid }\end{array}$ & $\begin{array}{c}\text { Chain Length } \\
\left(C_{n}\right)\end{array}$ & $\begin{array}{c}\text { Oven/Colume Temp } \\
\left({ }^{\circ} \mathrm{C}\right)\end{array}$ & $\begin{array}{l}\text { Retention Time } \\
\text { (min) }\end{array}$ \\
\hline Stearic acid & 18 & 180 & 6.17 \\
\hline Palmitic acid & 16 & 180 & 3.18 \\
\hline Myristic acid & 14 & 180 & 1.71 \\
\hline Lauric acid & 12 & 160 & 1.53 \\
\hline
\end{tabular}


Figure 6-3. Adsorption-association Isotherm of Fatty Acids with SV and ML Lactose Monohydrate. (A) Stearic acid; (B) Palmitic acid; (C) Myristic acid; and (D) Lauric acid.
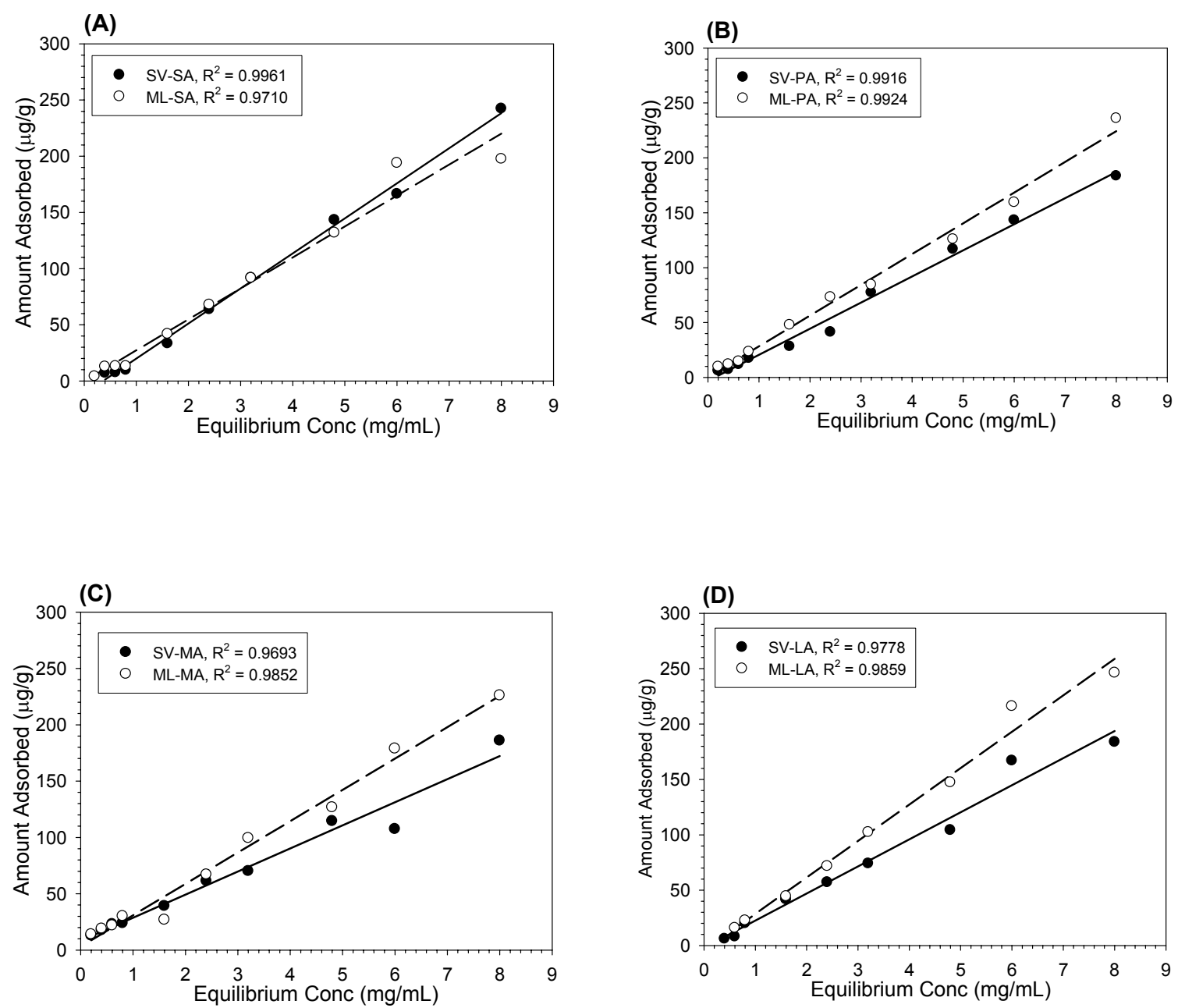
Figure 6-4. Differential Scanning Calorimetry thermograms of Lactose Monohydrate after Fatty Acid Surface Coating: (A) -SVcontrol $\left(\mathrm{CH}_{2} \mathrm{Cl}_{2} / \mathrm{CHCl}_{3}\right.$ treated); SVlow ( 6 $\mu \mathrm{g} / \mathrm{g}$ stearic acid); SVhigh ( 140 $\mu \mathrm{g} / \mathrm{g}$ stearic acid); (B) -MLcontrol $\left(\mathrm{CH}_{2} \mathrm{Cl}_{2} / \mathrm{CHCl}_{3}\right.$ treated); MLlow; MLhigh; (C) SV-AS; (D) ML-AS; (E) SV-FP; (F) ML-FP .
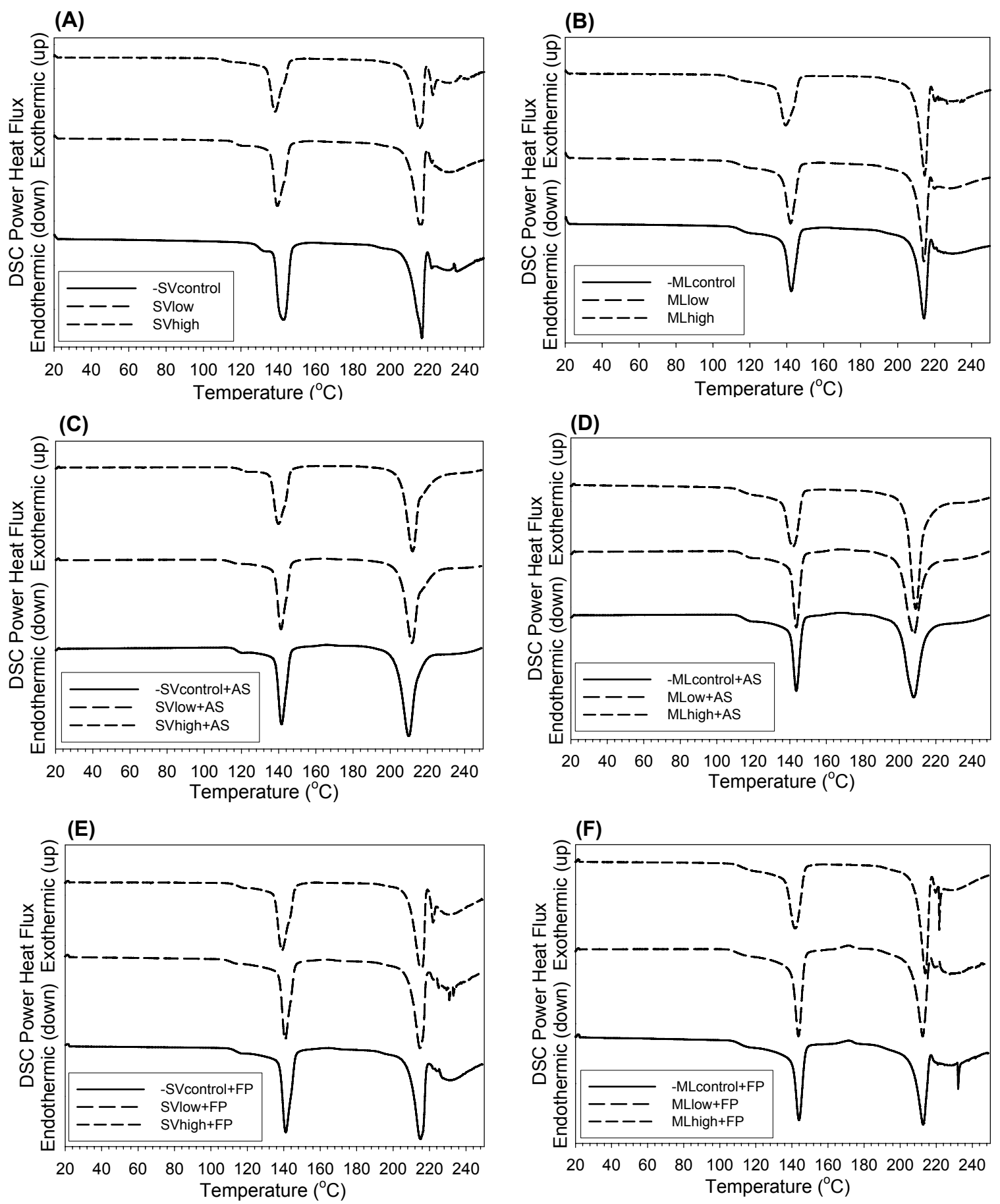
Figure 6-5. PADE Nonlinear Regression and Linear Regression of AS Formulations.
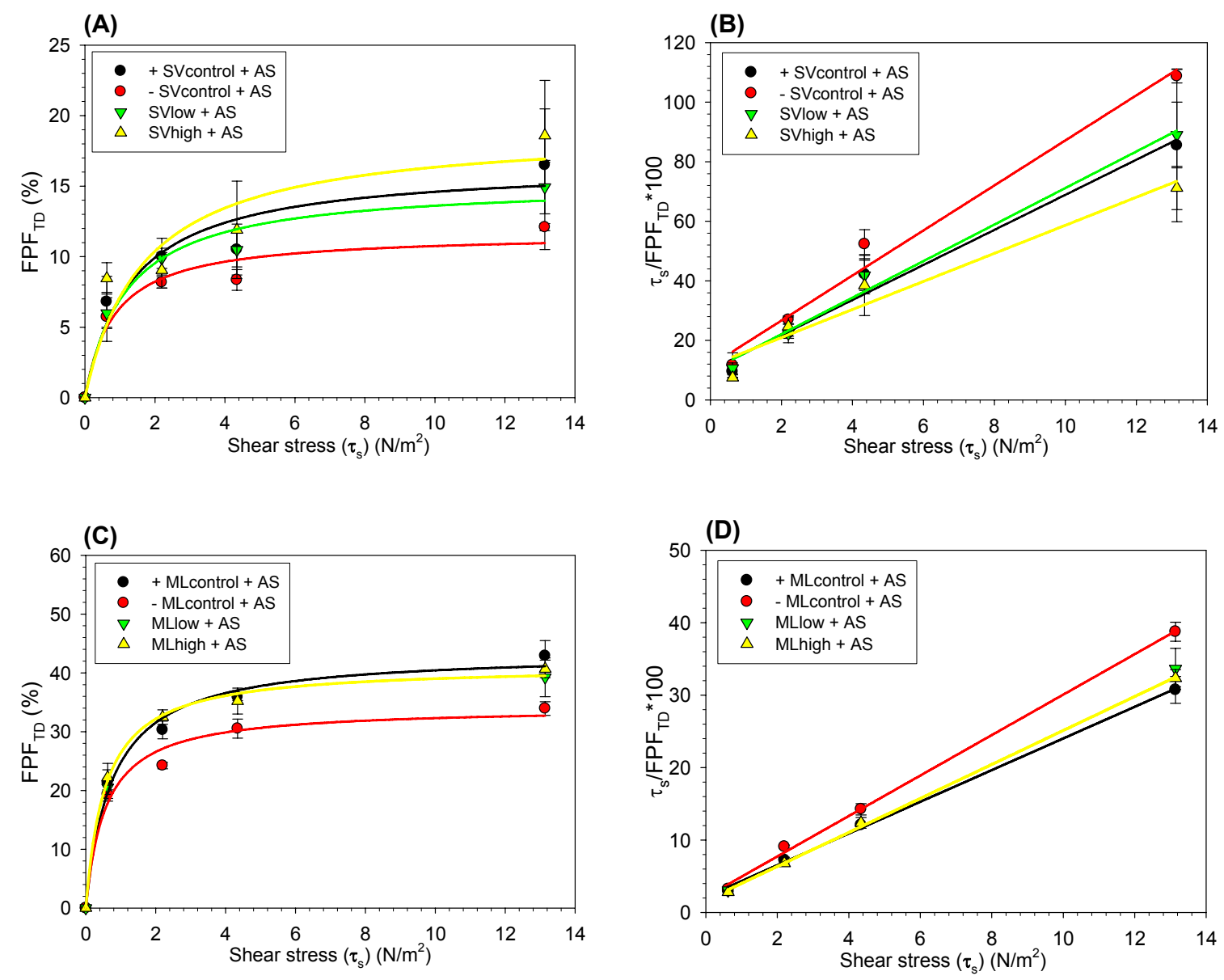
Figure 6-6. PADE Nonlinear Regression and Linear Regression of FP Formulations.
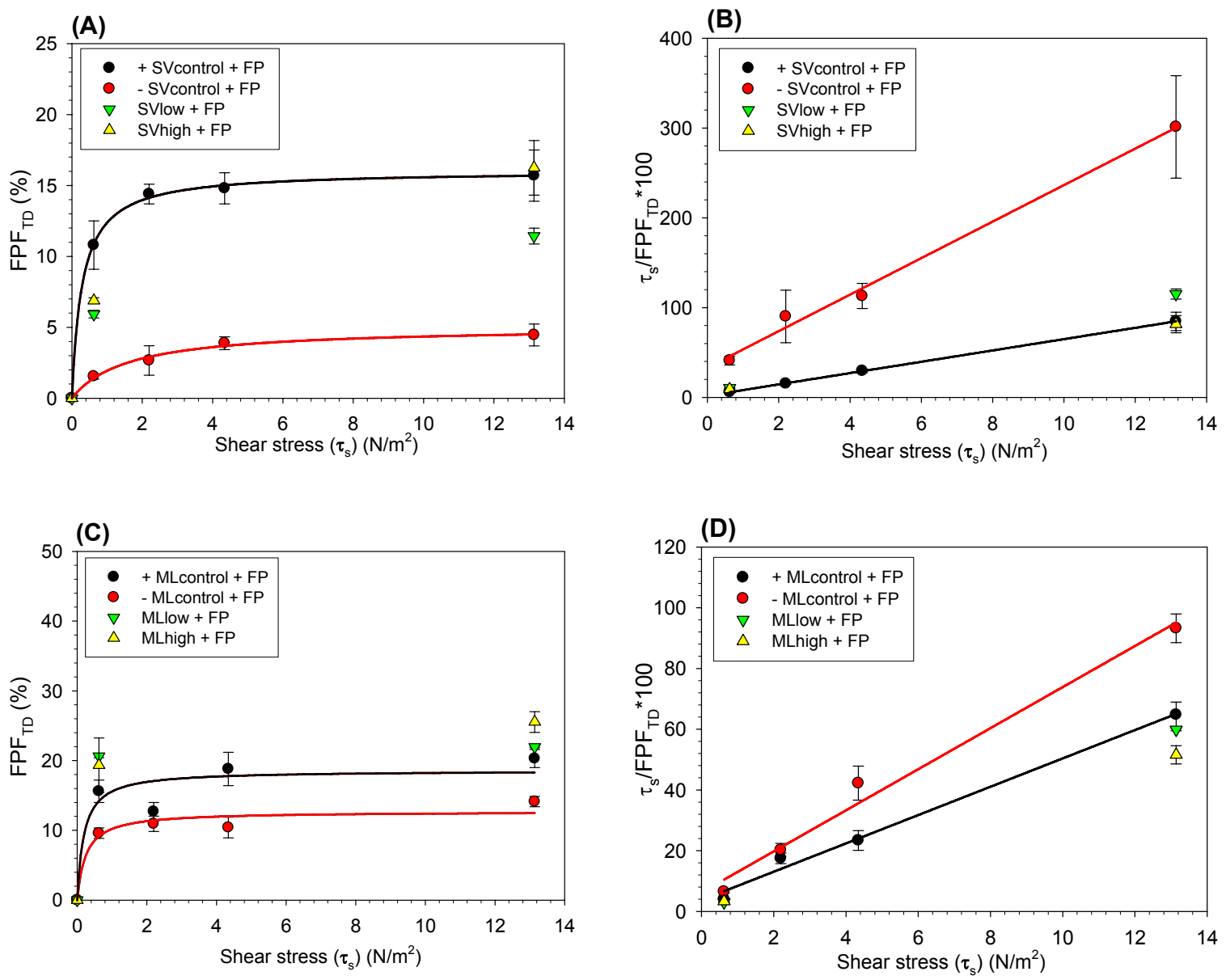
Table 6-1. Physical Characterization of the Powder Flow Properties ( $n=3$, mean value)

\begin{tabular}{|c|c|c|c|}
\hline & $\begin{array}{c}\text { Bulk density } \\
\left(\mathbf{k g} / \mathrm{m}^{3}\right)\end{array}$ & $\begin{array}{c}\text { Tap density } \\
\left(\mathrm{kg} / \mathrm{m}^{3}\right)\end{array}$ & Carr's index \\
\hline+ SV control & 659 & 890 & 0.26 \\
\hline -SVcontrol & 589 & 866 & 0.32 \\
\hline SVlow & 606 & 866 & 0.30 \\
\hline SVhigh & 684 & 877 & 0.22 \\
\hline +MLcontrol & 555 & 925 & 0.40 \\
\hline -MLcontrol & 548 & 944 & 0.42 \\
\hline MLlow & 554 & 923 & 0.40 \\
\hline MLhigh & 595 & 933 & 0.36 \\
\hline
\end{tabular}




\section{References}

1. A.J. Hickey and T.B. Martonen, Pharm Res, 10, 1-7 (1993).

2. J. Yang, A. Sliva, A. Banerjee, R.N. Dave and R. Pfeffer, Powder Tech., 158, 21-33 (2005).

3. M.J. Clarke, M.J. Tobyn and J.N. Staniforth, J Pharm Sci, 90, 213-23 (2001).

4. P. Begat, R. Price, H. Harris, D.A. Morton and J.N. Staniforth, Kona, 23, 109-121 (2005).

5. M. Kumon, M. Suzuki, A. Kusai, E. Yonemochi and K. Terada, Chem Pharm Bull (Tokyo), 54, 1508-1514 (2006).

6. K. Iida, Y. Hayakawa, H. Okamoto, K. Danjo and H. Luenberger, Chem Pharm Bull (Tokyo), 52, 444-446 (2004).

7. K. Iida, Y. Hayakawa, H. Okamoto, K. Danjo and H. Luenberger, Chem Pharm Bull (Tokyo), 52, 350-353 (2004).

8. K. Iida, Y. Hayakawa, H. Okamoto, K. Danjo and H. Luenberger, Chem Pharm Bull (Tokyo), 51, 1455-1457 (2003).

9. M.D. Jones and R. Price, Pharm Res, 23, 1665-1674 (2006).

10. N. Islam, P. Stewart, I. Larson and P. Hartley, Pharm Res, 21, 492-499 (2004).

11. P.M. Young, H.K. Chan, H. Chiou, S. Edge, T.H. Tee and D. Traini, J Pharm Sci, 96, 1331-1341 (2007).

12. D. Ganderton, J Biopharm Sci, 3, 101-105 (1992).

13. M. Sacchetti, Pharm Dev Technol, 11, 351-358 (2006).

14. R. Price, P.M. Young, S. Edge and J.N. Staniforth, Int J Pharm, 246, 47-59 (2002).

15. P.M. Young, S. Edge, D. Traini, M.D. Jones, R. Price, D. El-Sabawi, C. Urry and C. Smith, Int J Pharm, 296, 26-33 (2005).

16. S. Ward, M. Perkins, J. Zhang, C.J. Roberts, C.E. Madden, S.Y. Luk, N. Patel and S.J. Ebbens, Pharm Res, 22, 1195-1202 (2005).

17. P. Young and R. Price, Respiratory Drug Delivery, 1, 327-332 (2006). 
18. X.M. Zeng, G.P. Martin, S.K. Tee, A.A. Ghoush and C. Marriott, Int J Pharm, 182, 133-144 (1999).

19. P. Lucas, K. Anderson and J.N. Staniforth, Pharm Res, 15, 562-569 (1998).

20. M.D. Louey and P.J. Stewart, Pharm Res, 19, 1524-1531 (2002).

21. H.K. Chan, J Aerosol Med, 19, 21-27 (2006).

22. N.M. Kassem and D. Ganderton, J Pharm Pharmacol, 41, 14P (1989).

23. H. Adi, I. Larson, H. Chiou, P. Young, D. Traini and P. Stewart, Pharm Res, 23, 2556-2565 (2006).

24. M.D. Jones, J.C. Hooton, M.L. Dawson, A.R. Ferrie and R. Price, Pharm Res, 25, 337-348 (2008).

25. D. Cline and R. Dalby, Pharm Res, 19, 1274-1277 (2002).

26. F. Podczeck, J Adhesion Sci Tech, 12, 1323-1339 (1998).

27. J.D. Lord and J.N. Staniforth, Particle size effects on packing and dispersion of powders. Interpharm, (1996).

28. X.M. Zeng, G.P. Martin, S.K. Tee and C. Marriott, Int J Pharm, 176, 99-110 (1998).

29. X.M. Zeng, G.P. Martin, C. Marriott and J. Pritchard, J Pharm Sci, 90, 1424-1434 (2001).

30. M. Gotzinger and W. Peukert, Langmuir, 20, 5298-303 (2004).

31. M.D. Louey, P. Mulvaney and P.J. Stewart, J Pharm Biomed Anal, 25, 559-67 (2001).

32. H.Y. Erbil, Surface chemistry: solid and liquid interfaces. Blackwell publishing Ltd, Malden, MA (2006).

33. D.D. Do, Adsorption analysis: equilibria and kinetics. Imperial College Press, London, UK (1998).

34. Y. Kawashima, T. Serigano, T. Hino, H. Yamamoto and H. Takeuchi, Int J Pharm, 172, 179-188 (1998). 
35. P. Begat, D.A. Morton, J. Shur, P. Kippax, J.N. Staniforth and R. Price, J Pharm Sci, 98, 2770-83 (2009).

36. A.J. Hickey, G.V. Jackson and F.J. Fildes, J Pharm Sci, 77, 804-9 (1988).

37. K.A. Fults, I.F. Miller and A.J. Hickey, Pharm Dev Technol, 2, $67-79$ (1997).

38. D.J. Cooney and A.J. Hickey, J Pharm Sci, 92, 2341-4 (2003).

39. H. Ichikawa and Y. Fukumori, Int J Pharm, 180, 195-210 (1999).

40. P. Lucas, K. Anderson, U.J. Potter and J.N. Staniforth, Pharm Res, 16, 1643-1647 (1999).

41. M. Linsenbuhler and K.E. Wirth, Powder Technol., 158, 3-20 (2005).

42. J. Raula, F. Thielmann, J. Kansikas, S. Hietala, M. Annala, J. Seppala, A. Lahde and E.I. Kauppinen, Pharm Res, 25, 2250-2261 (2008).

43. A. Lahde, J. Raula and E.I. Kauppinen, Int J Pharm, 358, 256-62 (2008).

44. A.J. Hickey, I. Gonda, W.J. Irwin and F.J. Fildes, J Pharm Sci, 79, 1009-14 (1990).

45. M. Kumon, S. Machida, M. Suzuki, A. Kusai, E. Yonemochi and K. Terada, Chem Pharm Bull (Tokyo), 56, 617-625 (2008).

46. Future Digital Scientific Corp.data. Accessed in 2009 [Available from: http://www.fdsc.com/.

47. G.E. Amidon, in: Physical Characterization of Pharmaceutical Solids, H.G. Brittain, Vol. 70, pp. 281-319. Marcel Dekker, New York (1995).

48. S. Yokoyama, Y. Fujino and T. Fujie, Chem Pharm Bull (Tokyo), 41, 2026-2028 (1993).

49. S. Yokoyama and T. Fujie, Chem Pharm Bull (Tokyo), 38, 2249-2252 (1990).

50. C. Crim, L.N. Pierre and P.T. Daley-Yates, Clin Ther, 23, 1339-54 (2001). 


\section{CHAPTER 7 GENERAL DISCUSSION}

\subsection{Review}

The delivery of aerosol drugs to the respiratory systems greatly improved the treatment of many diseases. Inflammatory airway diseases, such as asthma have been successfully treated using inhalation aerosols. Of the three types of aerosol delivery methods, dry powder inhalers (DPIs) are an alternative to the commercially dominant propellant-driven metered dose inhalers (MDIs) for inhalation therapy. With the manufacturing and engineering of inhalation particles growing as a research endeavor, the production of dry powders with desired particle size distributions, morphology, and other characteristics become possible. The major hurdles for effective treatment using DPIs relate to the formulation performance efficiency and reproducibility. Current device and formulation combinations often give sub-optimal performance and dosing variation. This is partly due to poor understanding of the relationship between the physicochemical nature of powder formulation and the behavior of particles in the aerosolization process. Some drugs such as $\beta_{2}$-agonists are widely accepted for inhalation due to their wide therapeutic windows. For these drugs, efficient devices and formulations may be a priority since they reduce the loss of drug and are cost effective. But drugs with narrower therapeutic windows, such as steroids, require more accurate drug delivery. For such drugs, dosing accuracy may be the major concern. From the pharmacological classification, five major types of therapeutics are used for the treatment of asthma and COPD. The consistent and efficient delivery of a reliable dose to the appropriate parts of the 
respiratory tract using DPIs requires a good understanding of the characteristics of static powder, the aerosol generation, the aerodynamic behavior of aerosols, the aerosol deposition mechanisms, and site of actions.

Dry powder formulations are mainly composed of micronized drug particles and larger carrier particles, typically lactose monohydrate. The factors that influence aerosolization performance are numerous. Great effort has been expended to address reproducible aerosolization and efficient performance by manipulating these factors, but with limited success. Although the calculated interparticulate adhesive and cohesive forces (van der Waals, electrostatic, and capillary forces) between ideal particles have been available for many years, these forces, measured experimentally, are not directly predictive of the aerosol performance. The difficulty lies in our current poor understanding and prediction of adhesive and aerodynamic forces and their effects on heterogeneous and multicomponent powder systems. Performance optimization remains a largely empirical process. Moreover, unlike the situation with liquid droplets, the particles in the solid state have limited molecular mobility. They are rigid and resistant to stress. The surface molecules of the solid cannot immediately rearrange to equilibrate with the bulk. Consequently, the surfaces of the particles are highly conditioned by their past history such as the method of particle formation, processing (milling and blending, filling), and storage conditions (RH and temperature) (Figure 7-1). The variation of these interparticulate interactions will subsequently dictate the aerodynamic performance of powder aerosols and therapeutic success.[1, 2] An overview of pharmaceutical processing that controls interparticulate interactions and performance is shown in Table 1-2.[3]. 


\subsubsection{Pharmaceutical Processing}

\subsubsection{Origins of particles: Milling and spray drying}

Bulk drugs must be prepared in suitable size ranges for aerosol delivery. A variety of conventional and innovative research methods have been developed for controlled aerosol generation and their theoretical principles have been readily established.[4] Among them, two most common methods of aerosol drug particle preparation are milling (destructive) [1] and spray drying (constructive).[5] The former involves particle reduction by pressure, friction, impact, or shear depending on the types of mills used.[1,6] Among them, jet mills and fluid energy mills are often used for drug particle micronization within the respirable range, $[7,8]$ whereas ball mills are often used for the treatment of carriers such as $\alpha$-lactose monohydrate. $[9,10]$ The jet-milling is operated at high pressure. The $\mathrm{N}_{2}$ supply from the feed and opposing jets caused the powder to impact with each other and against the sides of the milling chamber resulting in micronization of the bulk powder. The centrifugal force in the milling chamber and cyclone separation was used to collect micronized powder inside the milling chamber, cyclone separator, and the collection vessel. Superfine particles were trapped by a collection bag above the cyclone. The yield of micronized particles collected was $\sim 50-60 \%$.

Generally, milling has limited control of particle size distribution. The degree of surface amorphous content increases with a longer milling time[9, 11] and higher milling pressure [11]. Milling is accompanied by surface area and molecular disorder increase, and potential surface charge buildup, which may cause particle agglomeration. As processing 
technologies advance, some specialized milling process may achieve more controllable and predictable results. The latter is operated when a pressurized drug solution or suspension is atomized to a spray and then in contact with a hot drying airstream, resulting in rapid evaporation and drying of the droplet to form solid particle. These methods are relatively simple, cost-effective, and suitable for both small- and large-scale processing. From the perspective of conventional solid dosage forms such as capsules or tablets, these processes share similar intent at enhancing drug dissolution rate, diffusion, and absorption from sites. For pulmonary delivery, a more important purpose is to achieve a suitable aerodynamic particle size for site-specific aerosol delivery. $[1,12]$

The influence of milling of drugs or carriers on the aerosol performance has been studied extensively [8, 9, 13-17]. Aerosol dispersion of mannitol microparticles produced by jet milling and spray-drying was compared and greater aerosol dispersion was observed for spray-dried particles [8]. This was attributed to reduced van der Waals forces due to particle shape and reduced electrostatic forces due to the particle generation [8]. The performance of an inhalation peptide, cetrorelix, was also examined using milling and several other particle engineering techniques [15]. The fine particle fraction (FPF, particles with aerodynamic diameter $<6.8 \mu \mathrm{m}$ sampled at $90 \mathrm{~L} / \mathrm{min}$ ) was lower for milled cetrorelix than spray dried or spray freeze dried particles. However, in another study, with tuned pearl milling of cetrorelix, a substantial increase of FPF $(\sim 60 \%)$, much higher than spray dried formulation performance could be achieved [16]. The study of milling effects of carrier lactose monohydrate on formulation performance showed that higher milling intensity and increased mill time resulted in higher FPF of a variety of drugs delivered from blends with these lactose batches 
$[9,13]$. A linear relationship was observed when FPF was correlated with mill time [9]. A linear relationship between FPF and the intrinsic lactose fines content revealed that the fines generated from the milling process served as a ternary component and played a major role in improving in vitro performance $[9,13]$.

\subsubsection{Mixing}

In order to overcome agglomeration and increase powder flow properties, achieve content uniformity and accurate dosing, micronized drugs are often mixed with coarser carrier particles. Mixing is a statistical process, involves relative displacement of particles of one component with another component until a state of uniformity is achieved. [1, 18] In practice, the degree of homogeneity can be estimated by evaluating the sample variance $\left(\sigma_{s}^{2}\right)$ (Eq. 7-1).[18]

$$
\sigma_{s}^{2}=\sum_{i=1}^{N} \frac{\left(x_{i}-\bar{x}_{s}\right)^{2}}{N-1}
$$

where $x_{i}(i=1,2, \ldots, \mathrm{N})$ is the composition in the $i$ th sample taken randomly (e.g. employing sample thieves [19]); $\overline{x_{s}}$ is the sample mean. The blend homogeneity is an important indicator of uniform mixing. A detailed discussion of sampling methods and homogeneity analysis was reviewed by a few research groups.[20-23] The mechanisms of mixing include convective (large-scale random motion), diffusive (small-scale random motion) and shear (momentum exchange due to velocity distribution) mixing depending on the mixer and mixing time. $[1,18]$ In theory, mixing is a time-dependent process following an exponential 
rise.[1] The mixing rate can be expressed by a first order kinetics as Eq. 7-2, where the mixing index, $M$, is defined as Eq. 7-3:[1]

$$
\begin{gathered}
M=1-e^{-k t}(\text { Eq. } 7-2) \\
M=\frac{s_{r}}{s}(\text { Eq. } 7-3)
\end{gathered}
$$

where $k$ is the rate constant $\left(\mathrm{s}^{-1}\right)$ dependent on the material, mixer geometry and operation; $s_{r}$ represents the standard deviation of a series of samples drawn from a random mixture; $s$ is the standard deviation of samples drawn from the mixture under examination. In reality, the rate constant $k$ is affected appreciably by the material, mixer geometry, and mixing intensity and mechanism $[1,24-26]$.

The micronized drug particles are generally cohesive, which may affect the mixing efficiency.[27, 28] The carrier particles are significantly larger than drug particles so that an even coating of drug particles may surround the carrier particles to form the so-called ordered mixing $[29,30]$. Due to the particle size distribution of both respirable drug and carrier particles, both randomization and ordering are present in equilibrium [31]. A narrower size distribution of carrier particles can greatly increase the mixing efficiency and avoid segregation, which is known as the competing mechanism and opposite of mixing [31, 32].

Batch to batch variations in blend homogeneity will affect aerosol performance. The effects of concentration and material properties on multicomonent mixing of cohesive powders were evaluated previously [33]. The variables that can be manipulated to influence drug-carrier interaction in mixing process were reviewed [34]. They include the degree of fine particle 
break-up during mixing, drug distribution, interaction forces, and press-on forces between drug and carrier particles [34]. The blending sequence may also influence the powder performance [35]. In recent years, there has been great interest in the development of ternary mixing systems because the inclusion of ternary component into the carrier-based dry powder formulation results in improved formulation performance due to a drug-carrier interaction change [36-38]. In the lactose-based DPI formulations, such ternary components can be fines of $\alpha$-lactose monohydrate or alternative sugars [36, 39], or intrinsic fines generated from the primary lactose monohydrate particles [40]; or a variety of force control agents such as magnesium stearate [41-43], leucine [44] and trileucine [45]; nanoparticles [46, 47]; polymers [48] or active drugs [49]. Based on the effect of fines on the aerosolization performance, two different hypotheses were proposed to account for performance improvement of carrier-based dry powder formulation: the "active" site and agglomeration hypotheses (see Figure 6-1).[36]

\subsubsection{Filling and storage}

The filling of DPI formulation for aerosol product is a challenging task. This is partly because the lack of lung administration safety data restricted the use of many excipients, diluents, lubricants, binders, and surfactants in filling that are commonly used in oral products. $\alpha$-Lactose monohydrate acts as excipient, diluent, and carrier. Besides the conventional requirement for powder filling including accuracy, consistency, and uniformity in standard pharmaceutical dosage forms (capsule, blister, strip, reservoir), the challenges for DPI powder filling include low apparent density, particulate cohesion and adhesion, low 
filling weight, and high-throughput filling. Similar problems encountered in mixing such as percolation and trajectory segregation should also be considered.

Powder product stability is an issue of storage over time. A thermodynamically unstable molecular disorder may be created by the above mentioned processes. The unstable form may undergo molecular relaxation such as recrystallization and/or particle fusion resulting in poor aerosolization.[50] For example, the recrystallization of amorphous albuterol sulfate can happen during the process of milling or blending, leading to particle growth, agglomeration and size distribution shift.[51, 52] Moisture adsorption and ingress in the amorphous region due to increased water activity and capillary condensation will cause agglomeration and particle bridging, resulting in humidity-induced aerodynamic property change in dry powder formulations.[53, 54]

\subsubsection{Summary of Findings}

This dissertation addressed the frequently encountered problems during DPI development: the delivery efficiency and dosing variability. In order to improve performance and optimize the DPI formulations, the heterogeneous nature of carrier-based DPI formulations was systematically investigated. The physicochemical properties of one-component (drug or carrier alone) and two-component (interactive physical mixtures of different drugs and carriers) systems, and their aerodynamic performance were characterized.

For most of the aerosolization performance studies, SETs were applied. The mechanisms of particle separation in the SETs include 1) particle entrainment in a laminar or 
turbulent shear fluidization; 2) particle entrainment in a laminar or turbulent wall boundary layer; 3) turbulent deaggregation of agglomerates by aerodynamic forces or turbulent transient acceleration (relative motion) and; 4) particle detachment by momentum transfer (e.g. impaction and vibration). These mechanisms manifested to a different extent in the SETs and all of them are directly related to the fluid energy dissipated per unit volume of the flow, $\varepsilon$. The adequate measure of such dissipation is the magnitude of viscous turbulent shear, $\tau_{s}$. This parameter varies within the flow cross-section and also depends on the flow geometry, pressure drop, and Reynolds number. The studies using SETs minimized the confounding factors caused by inhaler devices and allowed for studies that could focus on the relationship of airflow conditions and formulation performance.

In order to elucidate the relationship between the dry powder formulations and their aerosolization performance, the performance data were correlated with the aerodynamic parameters (represented as $\tau_{s}$ ). Excellent correlation results were observed in all studied formulations. A novel interpretation using powder aerosol deaggregation (PADE) was applied to describe and compare the particulate surface dissociation across a range of shear stress.[55-58] Briefly, PADE is a method that developed based on the fundamental understanding that the forces acting at the particle interface are analogous to those that the molecular level, and that models of molecular surface association described by an adsorption expression can be adapted to fit shear displacement observations. In principle, drug particles adhere to the carrier surface by fundamental forces including van der Waals, electrostatic and capillary interactions. As shear forces apply to the surface of a drug coated carrier particle, drug particles are removed with increasing difficulty because of the sites that they occupy 
until a saturation is reached, when no drug particle can be removed at increasing shear forces unless comminution of particles. The analogy from surface adsorption/desorption leads to a completely novel way of looking at particle interactions. This method is significant because it not only describes the formulation performance across the entire shear stress range, but more importantly may reveal the mechanism of drug deaggregation during inhalation. In the PADE non-linear regression analysis, the invariable (shear independent) region at high shear has important predictive implications for screening (performance evaluation) of existing formulations and rationally designing of new formulations. It will be easy to idientify the optimum shear required for efficient deaggregation close to the plateau, yet within the specific resistance limit for patient's inhalation. The variable (shear dependent) region at lower shear reflected in the specific interfacial and physicochemical properties of drugs and carriers in their heterogeneous state. It has the potential to reveal the mechanisms of drug deaggregation considering the surface energy heterogeneity and lateral interaction forces. This can be achieved by increasing the dataset for PADE non-linear curve fitting of different algebraic equivalent surface adsorption models and use statistical thermodynamic approach to particle deaggregation studies analogous to those used in adsorption theory.

During the development of the PADE method, three apparently similar but inherently different surface phenomena, adsorption, protein binding, and particle disassociation were compared. These surface phenomena are governed by the same fundamental forces but different dimensions and magnitude (i.e. intermolecular, colloidal, and interparticulate). They share the same algebraic expression that could be derived thermodynamically from the surface energy. The interfacial driving forces also share the 
same unit $\left(\mathrm{N} / \mathrm{m}^{2}\right)$ between adsorption and deaggregation. Real systems of all these surface phenomena require the consideration of surface heterogeneity, various binding affinity and sites, and lateral interactions. Among them, the adsorption theory (e.g. Langmuir adsorption) equates the rates of adsorption and desorption in terms of fraction loading. The dynamic equilibrium is time independent. At high pressure, the amount adsorbed reaches the saturation capacity to form a monolayer surface coverage. The treatment of heterogeneous adsorption is achieved by grouping the similar surface energy sites in a patchwise or random manner using statistical thermodynamic approach and applies appropriate isotherm to each local adsorption. Similarly, the protein binding is also a time independent equilibrium process. The amount of adsorbed and monolayer surface coverage in langmuir adsorption are now described as the percentage of drug bound in the total protein and the number of independent binding sites, respectively. The number of binding sites determines the saturation capacity. If there're multiple binding sites or binding sites are dependent one another (heterogeneity), the treatment is usually to group similar classes of binding sites, treat each class of protein binding separately and then add up. As an analogy to the surface coverage in adsorption and the number of independent binding sites in protein binding, the parameter in deaggregation expression is defined as the maximum achievable fine particle fraction $\left(F P F_{\max }\right)$. The heterogeneity in particle deaggregation is probably the most complicated because geometric, energetic characteristics of both drug particle and carrier particle, and the interfacial interaction of both particles contribute to the heterogeneity. The analogy to surface adsorption expressions was eventually chosen. 
Despite the unifying concepts between these surface phenomena, there are fundamental differences between the powder deaggregation and the other two concepts. The physical adsorption is a molecular thermodynamic diffusion process. The residence time (time required for molecular transport to adsorption sites) is relatively short. However, the actuation of dry powder to form aerosols is most likely a different scenario. Because of the transient nature of powder entrainment and deaggregation (a short burst of powder), the particle deaggregation in this manner should be differentiated from the long-term particle diffusion by a time independent "particle desorption/deposition" model which could be viewed as a true analogy to adsorption phenomena.[59, 60] The particle entrainment and deaggregation, due to the particle size and system dimension, cannot be treated as a continuum for continued drug particle adhesion and deaggregation. Furthermore, the particle deaggregation in a burst involves rapid system volume expansion and the corresponding net gain in entropy.

The newly developed PADE method was subjected to several types of evaluation. They include the performance evaluation of a variety of carrier-based formulations (different drugs or carriers), the performance comparison of SETs vs. DPI devices, the storage effects to the performance efficiency. These applications proved PADE as a robust formulation optimization and screening tool in the defined shear range.

As an analogy to Langmuir adsorption theory, the PADE method tolerated well in the heterogeneous particulate systems containing both drug and carrier particles. It was hypothesized that by applying a force control agent (fatty acid) coating, the surface heterogeneity could be reduced and the performance efficiency could be improved. The 
surface coating could potentially mask the high surface energy density sites and could serve as a probe for mechanistic study of PADE. It was found the analogous "surface occupancy" approach described by adsorption theory could potentially account for both proposed "active" site (e.g. Langmuir type) and "agglomeration" (e.g. B.E.T. type) and without favoring either mechanism. By using similar surface adsorption expression for aerosol performance, it is conceivable that an analogous mechanistic approach to the evaluation of particle-particle and particle surface interactions might be achieved.

\subsection{General Conclusion}

The mechanics of DPIs are complex and not very well understood, largely because of the heterogeneous nature of dry powders and the turbulent nature of airflow in which the aerosols are entrained and resuspended. Using SETs in which particle separation occurs in response to shear stress, we have developed a basic understanding of aerosolization performance described by PADE. To our knowledge this is the first time that such an approach has been suggested. It is conceivable that particles which can be stripped by shear forces may more readily be removed under the influence of impaction and momentum transfer, from which it can be inferred that these formulation performance studies may extrapolate qualitatively to drug product performance. This observation may serve as a link between macroscopic statistical thermodynamics and microscopic individual particle deaggregation. Dry powder deaggregation is a very complex process. The surface imperfection including geometric and energetic heterogeneity, lateral interaction (drug-drug cohesion), the nonideality of the bulk, and the turbulent airflow conditions, all influence the 
macroscopic aerosolization. But this complex deaggregation process can now simply be described by adapting surface adsorption theories and fitting the shear displacement using SETs. The PADE method is served to open a new window for the novel interpretation of aerosol formulation development.

\subsection{Future Work}

A broad range of future studies may be conducted to support the general application of the PADE.

1) The Langmuir adsorption isotherm is the cornerstone of nearly all the surface adsorption expressions and has been most widely used. However, in many cases, the experimental results require adjustment that with respect to surface heterogeneity and lateral interactions. From a historical perspective, theoretical and semi-empirical expressions were based on experimental data and statistical thermodynamic derivation considering various influences such as affinity constant change (e.g. Volmer isotherm [61]), surface heterogeneity (e.g. Freundlich [62], Sips [63], Toth [64], Unilan [61] equation), and lateral interaction (e.g. Fowler-Guggenheim equation [61]). Likewise, the PADE (Langmuir equivalent) method tolerated the surface heterogeneity and lateral interactions. But elaborating such an approach would have the benefit of describing more accurately the magnitude of the interaction and the likely underlying mechanisms, based on inflections in FPF with respect to shear that could be probed, subsequently, by controlled experiments (highly engineered particles: e.g. narrow span, porous, smooth, etc). 
2) One of the difficulties in the development of PADE (Langmuir equivalent) was definition of the deaggregation constant $k$. As an energy function, this parameter could be affected by a variety of factors, such as the heat of adhesion, the energy density distribution of drugs and carriers. It remains to be seen if this parameter could be related to the surface energy or the adhesive and cohesive forces. Future research should employ surface analytical techniques such as AFM and IGC to account for the influence of formulation to the aerosolization performance. The fluid dynamic methods could also be an alternative technique for adhesive force measurement since it involves adhesion in a particle-fluid system.

3) In the previous studies, the experiments were designed to account for the influences of drug, carrier, and SET shear stress, while the drug concentration (drug to carrier ratio), which could be important, was omitted. But this factor should be included in the future studies. Expressions describing surface adsorption were intended to allow elucidation of mechanisms including using the Brunauer classification for reference, lateral interactions, multilayers and capillary condensation.[65] The evolution of the Brunauer Emmett Teller (BET) equation encompassed the Langmuir expression as a special case with the assumptions that the successive heat of adsorption for all layers except the first are equal to the heat of condensation of the adsorbate (energetically homogeneous).[66, 67] Similar approaches were described by Giles et al. for solute adsorption at solid surfaces from solution resulting in a range of isotherms equivalent to the Brunauer classification.[68, 69]

4) From the ACI experimental data, it indicated that an exponential decay relationship could fit the data (analysis was not shown) when another fundamental parameter, MMAD, was correlated with shear stress. This was consistent with the earlier findings.[70] It was 
conceivable that a complete deaggregation of drug particles would produce an MMAD value equivalent to the aerodynamic diameter of the primary drug particles.

5) In addition to the turbulent shear forces which are the principal mechanism of powder entrainment and drug deaggregation, the impaction and momentum transfer are perhaps the most important mechanisms for deaggregation. Besides, frictional forces should be considered at initiation of powder entrainment. Their roles should be considered in the future. A computational fluid dynamics study of the SETs may be helpful.

6) It is worth investigating if the PADE method could be applied to other aerosol systems because only carrier-based formulations were evaluated. Further investigations of other aerosol systems such as carrier-free formulations, porous particle formulations, drug-carrier composite formulations, or even pMDI and nebulizer formulations are necessary. 
Figure 7-1 Pharmaceutical Dry Powder Aerosol Processing and Variables Associated with Each Process.
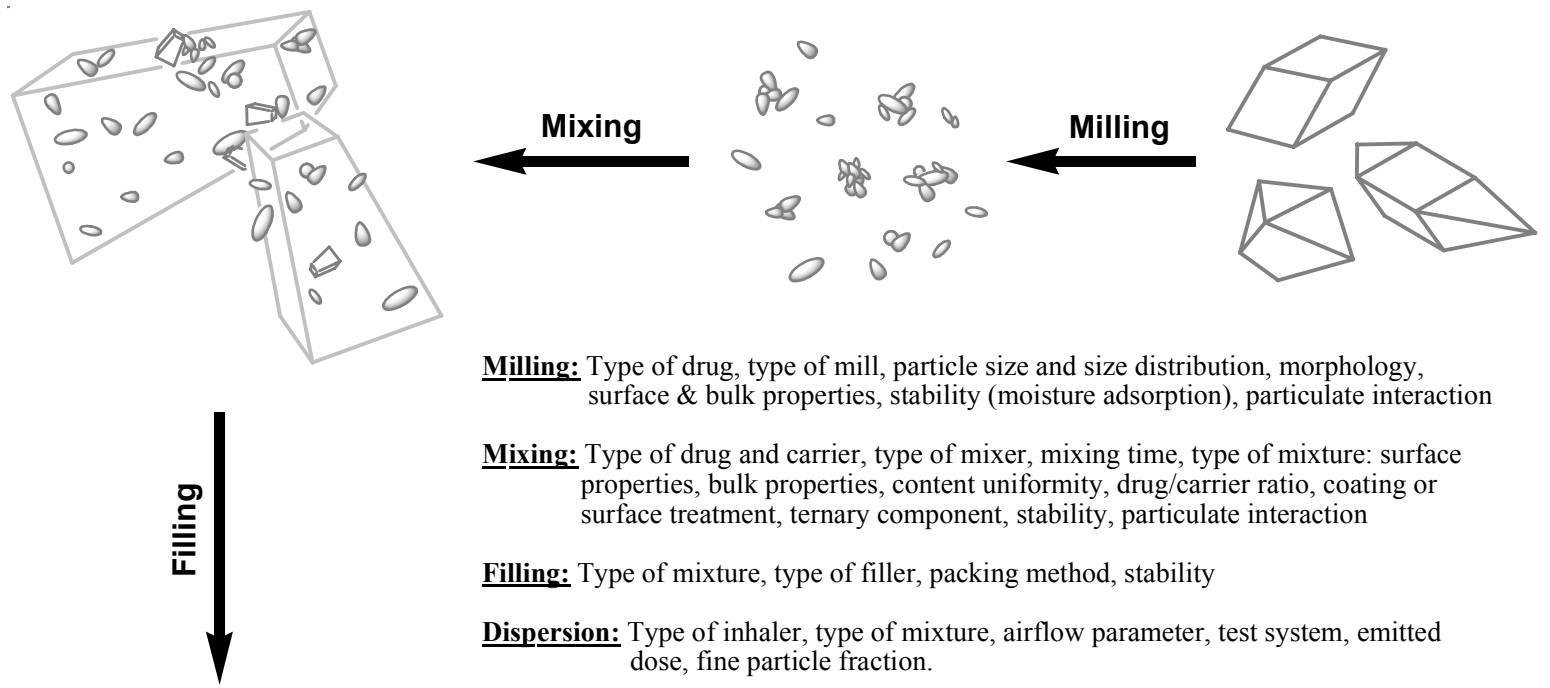

Milling: Type of drug, type of mill, particle size and size distribution, morphology, surface \& bulk properties, stability (moisture adsorption), particulate interaction

Mixing: Type of drug and carrier, type of mixer, mixing time, type of mixture: surface properties, bulk properties, content uniformity, drug/carrier ratio, coating or surface treatment, ternary component, stability, particulate interaction

Filling: Type of mixture, type of filler, packing method, stability

Dispersion: Type of inhaler, type of mixture, airflow parameter, test system, emitted dose, fine particle fraction.
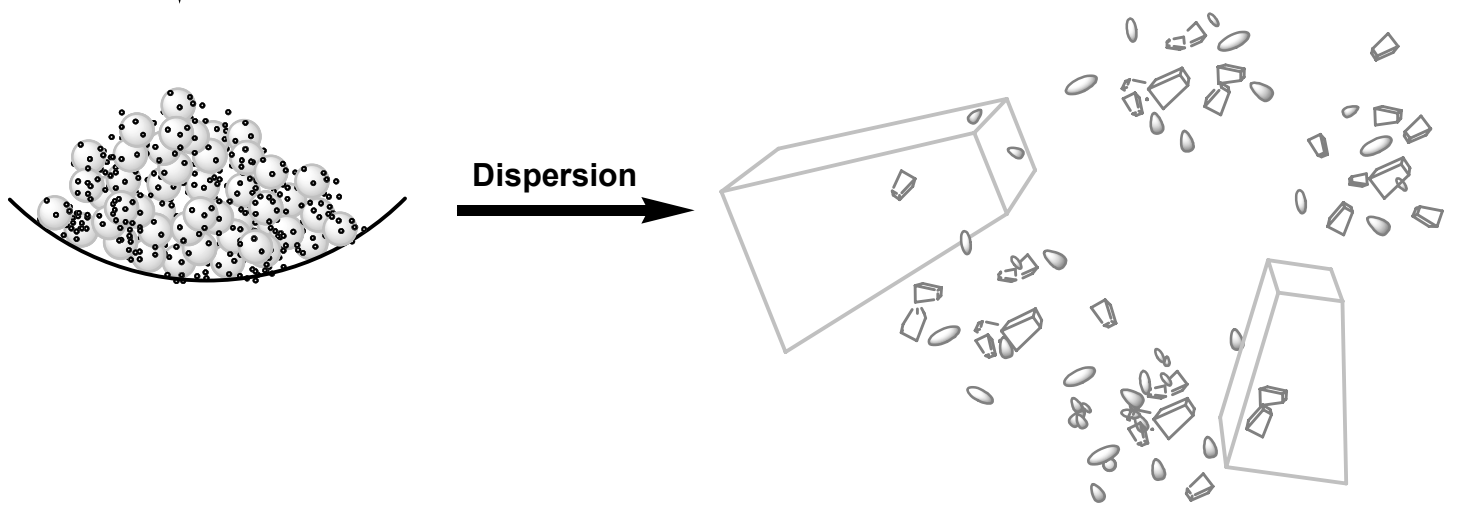


\section{References}

1. A.J. Hickey and D. Ganderton, Pharmaceutical Process Engineering. Marcel Dekker, New York (2001).

2. R. Dixit and S. Puthli, J Pharm Sci, 98, 3933-60 (2009).

3. Z. Xu, H.M. Mansour and A.J. Hickey, J Adhesion Sci Tech, In peer review (2010).

4. K.H. Leong, in: Pharmaceutical inhalation aerosol technology, A.J. Hickey (Eds.), Vol. pp. 253-278. Marcel Dekker, New York (2004).

5. M. Van Oort and M. Sacchetti, in: Inhalation Aerosols: Physical and Biological Basis for Therapy, A.J. Hickey (Eds.), Vol. 1, pp. 307-346. Informa Healthcare, New York (2007).

6. J.T. Carstensen, Pharmaceutical Principles of Solid Dosage Forms. Technomic Publishing Company, Lancaster, PA (1993).

7. $\quad$ N. Rasenack and B.W. Muller, Pharm Dev Technol, 9, 1-13 (2004).

8. M.D. Louey, M. Van Oort and A.J. Hickey, Pharm Res, 21, 1200-1206 (2004).

9. P.M. Young, H.K. Chan, H. Chiou, S. Edge, T.H. Tee and D. Traini, J Pharm Sci, 96, 1331-1341 (2007).

10. Y. Chen, Y. Ding, D.G. Papadopoulos and M. Ghadiri, J Pharm Sci, 93, 886-895 (2004).

11. K. Brodka-Pfeiffer, P. Langguth, P. Grass and H. Hausler, Eur J Pharm Biopharm, 56, 393-400 (2003).

12. E.S. Fisher, in: Encyclopedia of Pharmaceutical Technology, J. Swarbrick, Vol. 4, pp. 2339-2351. Informa Healthcare, USA, New York (2007).

13. H. Steckel, P. Markefka, H. teWierik and R. Kammelar, Int J Pharm, 309, 51-59 (2006).

14. K. Giry, J.M. Pean, L. Giraud, S. Marsas, H. Rolland and P. Wuthrich, Int J Pharm, 321, 162-166 (2006).

15. G.S. Zijlstra, W.L. Hinrichs, A.H. de Boer and H.W. Frijlink, Eur J Pharm Sci, 23, 139-149 (2004). 
16. M. Irngartinger, V. Camuglia, M. Damm, J. Goede and H.W. Frijlink, Eur J Pharm Biopharm, 58, 7-14 (2004).

17. H. Steckel, N. Rasenack, P. Villax and B.W. Muller, Int J Pharm, 258, 65-75 (2003).

18. K. Miyanami, in: Powder Technology Handbook, H. Masuda, K. Higashitani, and H. Yoshida, Vol. 1, pp. 577-589. Taylor \& Francis Group, Boca Raton, FL (2006).

19. F.J. Muzzio, P. Robinson, C. Wightman and D. Brone, Int J Pharm, 155, 153-178 (1997).

20. J. Berman, PDA J Pharm Sci Technol, 55, 209-222 (2001).

21. J. Berman, D.E. Elinski, C.R. Gonzales, J.D. Hofer, P.J. Jimenez, J.A. Planchard, R.J. Tlachac and P.F. Vogel, PDA J Pharm Sci Technol, 51 Suppl 3, S1-99 (1997).

22. G. Boehm, J. Clark, J. Dietrick, L. Foust, T. Garcia, M. Gavini, L. Gelber, J.M. Geoffroy, J. Hoblitzell, P. Jimenez, G. Mergen, F. Muzzio, J. Planchard, J. Prescott, J. Timmermans and N. Takiar, PDA J Pharm Sci Technol, 57, 64-74 (2003).

23. M. Howard-Sparks and A. Gawlikowski, PDA J Pharm Sci Technol, 58, 222-230 (2004).

24. M.M. de Villiers, Int J Pharm, 151, 1-6 (1997).

25. O.S. Sudah, D. Coffin-Beach and F.J. Muzzio, Int J Pharm, 247, 57-68 (2002).

26. I. Saleem, H. Smyth and M. Telko, Drug Dev Ind Pharm, 34, 1002-1010 (2008).

27. E.N. Hiestand, Mechanics and Physical Principles for Powders and Compacts. SSCI, West Lafayette, IN (2002).

28. F. Podczeck, Particle-particle Adhesion in Pharmaceutical Powder Handling. Imperial College Press, London, UK (1998).

29. J. Verraes and R. Kinget, J Pharm Belg, 34, 297-307 (1979).

30. J.A. Hersey, Powder Technol., 11, 41-44 (1975).

31. H.J. Venables and J.I. Wells, Drug Dev Ind Pharm, 27, 599-612 (2001).

32. K. Shinohara, in: Powder Technology Handbook, H. Masuda, K. Higashitani, and H. Yoshida (Eds.), Vol. 1, pp. 371-382. Taylor \& Francis Group, Boca Raton, FL (2006). 
33. Z.T. Chowhan, L. Chi and I. Yang, Powder Technol., 29, 251-256 (1981).

34. A.H. de Boer, P. Hagedoorn, D. Gjaltema, J. Goede, K.D. Kussendrager and H.W. Frijlink, Int J Pharm, 260, 201-216 (2003).

35. X.M. Zeng, G.P. Martin, S.K. Tee, A.A. Ghoush and C. Marriott, Int J Pharm, 182, 133-144 (1999).

36. M.D. Jones and R. Price, Pharm Res, 23, 1665-1674 (2006).

37. M.D. Jones, J.C. Hooton, M.L. Dawson, A.R. Ferrie and R. Price, Pharm Res, 25, 337-348 (2008).

38. K. Iida, H. Leuenberger, L.M. Fueg, R. Muller-Walz, H. Okamoto and K. Danjo, Yakugaku Zasshi, 120, 113-119 (2000).

39. M.D. Louey and P.J. Stewart, Pharm Res, 19, 1524-1531 (2002).

40. N. Islam, P. Stewart, I. Larson and P. Hartley, Pharm Res, 21, 492-499 (2004).

41. R. Guchardi, M. Frei, E. John and J.S. Kaerger, Int J Pharm, 348, 10-17 (2008).

42. P. Begat, R. Price, H. Harris, D.A. Morton and J.N. Staniforth, Kona, 23, 109-121 (2005).

43. F. Ferrari, D. Cocconi, R. Bettini, F. Giordano, P. Santi, M. Tobyn, R. Price, P. Young, C. Caramella and P. Colombo, AAPS PharmSciTech, 5, e60 (2004).

44. J. Raula, F. Thielmann, J. Kansikas, S. Hietala, M. Annala, J. Seppala, A. Lahde and E.I. Kauppinen, Pharm Res, 25, 2250-2261 (2008).

45. D. Lechuga-Ballesteros, C. Charan, C.L. Stults, C.L. Stevenson, D.P. Miller, R. Vehring, V. Tep and M.C. Kuo, J Pharm Sci, 97, 287-302 (2008).

46. M. Linsenbuhler and K.E. Wirth, Powder Technol., 158, 3-20 (2005).

47. A.S. Cantor, J.S. Stefely, P.A. Jinks, J.R. Baran, J.M. Ganser, M.W. Mueting, B.J. Gabrio and S.W. Stein, Respiratory Drug Delivery, 1, 309-318 (2008).

48. F. Buttini, P. Colombo, M.P. Wenger, P. Mesquida, C. Marriott and S.A. Jones, $J$ Pharm Sci, 97, 1257-1267 (2008).

49. G. Ward, Stabilized Dry Powder Formulations, WO World IPO 0170198. (2001). 
50. P.M. Young and R. Price, Eur J Pharm Sci, 22, 235-240 (2004).

51. G.H. Ward and R.K. Schultz, Pharm Res, 12, 773-779 (1995).

52. W.K. Ng, J.W. Kwek and R.B. Tan, Pharm Res, 25, 1175-1185 (2008).

53. C. Ahlneck and G. Zografi, Int J Pharm, 62, 87-95 (1990).

54. X.M. Zeng, H.B. MacRitchie, C. Marriott and G.P. Martin, Int J Pharm, 333, 45-55 (2007).

55. Z. Xu, H.M. Mansour, T. Mulder, R. McLean, J. Langridge and A.J. Hickey, J Pharm Sci, (2010 in press).

56. Z. Xu, H.M. Mansour, T. Mulder, R. McLean, J. Langridge and A.J. Hickey, J Pharm Sci, (2010 in press).

57. Z. Xu, H.M. Mansour, T. Mulder, R. McLean, J. Langridge and A.J. Hickey, J Pharm Sci, (2010 in press).

58. H.M. Mansour, Z. Xu and A.J. Hickey, J Pharm Sci, (2010 in press).

59. G. Ziskind, M. Fichman and C. Gutfinger, J. Aerosol Sci., 26, 613-644 (1995).

60. M.W. Reeks, J. Reed and D. Hall, J Phys D: Appl. Phys., 21, 574-589 (1988).

61. D.D. Do, Adsorption analysis: equilibria and kinetics. Imperial College Press, London, UK (1998).

62. H.M.F. Freundlich, J Phys Chem, 57, 385-470 (1906).

63. R. Sips, J Chem Phys, 16, 490-495 (1948).

64. J. Toth, Adv Colloid Interface Sci, 55, 1-239 (1995).

65. A.W. Adamson and A.P. Gast, Physical chemistry of Surfaces. John Wiley \& Sons, New York (1997).

66. S. Brunauer, P.H. Emmett and E. Teller, J Am Chem Soc, 60, 309-319 (1938).

67. T.L. Hill, J Chem Phys, 14, 263-267 (1946).

68. C.H. Giles, D. Smith and A. Huitson, J Colloid Interface Sci, 47, 755-765 (1974). 
69. C.H. Giles, D. Smith and A. Huitson, J Colloid Interface Sci, 47, 766-778 (1974).

70. M.D. Louey, M. VanOort and A.J. Hickey, J. Aerosol Sci., 37, 1520-1533 (2006). 
Appendix 1-A. Selected Dry Powder Inhalers (DPIs) and Their Characteristics

\begin{tabular}{|c|c|c|c|c|c|c|c|}
\hline Inhaler & Company & Drug & Carrier & $\begin{array}{l}\text { Powder } \\
\text { supply }\end{array}$ & Dosing & $\begin{array}{c}\text { Fluidization / } \\
\text { Deaggregation } \\
\end{array}$ & Diseases \\
\hline \multicolumn{8}{|c|}{ First generation: passive DPIs, single unit dose } \\
\hline Rotahaler & GSK & $\begin{array}{l}\text { AS, BDP, } \\
\text { SS+BDP }\end{array}$ & Lactose & Capsule & 1 & $\begin{array}{l}\text { Mechanical/ } \\
\text { turbulence }\end{array}$ & Asthma \\
\hline Inhalator & BI & FE, IPB & Glucose & Capsule & 1 or 6 & $\begin{array}{c}\text { Capillary/ } \\
\text { Shear force }\end{array}$ & Asthma \\
\hline Spinhaler & Aventis & DSCG & None & Capsule & 1 & $\begin{array}{l}\text { Capillary/ } \\
\text { Shear force }\end{array}$ & Asthma \\
\hline Cyclohaler & $\begin{array}{l}\text { Pharmach } \\
\text { emie }\end{array}$ & $\begin{array}{l}\text { SS, BDP, } \\
\text { IB, BUD }\end{array}$ & - & Capsule & 1 & 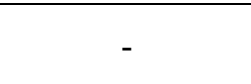 & Asthma \\
\hline Handihaler & $\mathrm{BI}$ & TI & Lactose & Capsule & 1 & - & COPD \\
\hline Aerolizer & Novartis & FO & & Capsule & 1 or 6 & $\begin{array}{l}\text { Capillary/ Shear } \\
\text { force }\end{array}$ & $\begin{array}{l}\text { Asthma, } \\
\text { COPD }\end{array}$ \\
\hline FlowCaps & Hovione & - & & Capsule & 1 & - & Asthma \\
\hline TwinCaps & Hovione & NI & & Capsule & 1 & - & Influenza \\
\hline \multicolumn{8}{|c|}{ Second generation: passive DPIs, multi-unit or multiple doses } \\
\hline Turbuhaler & $\mathrm{AZ}$ & $\begin{array}{l}\text { AS, TS, } \\
\text { BUD }\end{array}$ & None & Reservoir & 200 & $\begin{array}{l}\text { Shear, capillary } \\
\text { / shear force }\end{array}$ & Asthma \\
\hline Diskhaler & GSK & $\begin{array}{l}\text { SX, BDP, } \\
\text { FP, ZM }\end{array}$ & Lactose & Blister & 4,8 & $\begin{array}{c}\text { Shear force / } \\
\text { turbulence }\end{array}$ & $\begin{array}{l}\text { Asthma, } \\
\text { Influenza }\end{array}$ \\
\hline Diskus & GSK & $\begin{array}{c}\text { AS, SX, } \\
\text { FP, } \\
\text { SX+FP }\end{array}$ & Lactose & Strip & 60 & $\begin{array}{l}\text { Shear force / } \\
\text { turbulence }\end{array}$ & Asthma \\
\hline Aerohaler & $\mathrm{BI}$ & IPB & - & Capsule & 6 & & Asthma \\
\hline Easyhaler & $\begin{array}{l}\text { Orion } \\
\text { Pharma }\end{array}$ & $\mathrm{AS}, \mathrm{BDP}$ & Lactose & Reservoir & 200 & $\begin{array}{l}\text { Shear force / } \\
\text { turbulence }\end{array}$ & Asthma \\
\hline Ultrahaler & Aventis & - & - & Reservoir & - & - & Asthma \\
\hline Pulvinal & Chiesi & $\mathrm{AS}, \mathrm{BDP}$ & - & Reservoir & - & - & Asthma \\
\hline Novolizer & ASTA & BUD & Lactose & $\begin{array}{l}\text { Reservoir } \\
\text { cartridge }\end{array}$ & & & $\begin{array}{l}\text { Asthma, } \\
\text { COPD }\end{array}$ \\
\hline MAGhaler & $\mathrm{BI}$ & AS & Lactose & Reservoir & $\begin{array}{c}200- \\
500\end{array}$ & $\begin{array}{c}\text { Mechanical / } \\
\text { Mechanical }\end{array}$ & Asthma \\
\hline Taifun & $\begin{array}{l}\text { Focus } \\
\text { Inhal }\end{array}$ & AS & & Reservoir & & & Asthma \\
\hline Eclipse & Aventis & DSCG & & Capsule & & & Asthma \\
\hline Clickhaler & $\begin{array}{l}\text { Innoveta } \\
\text { Biomed }\end{array}$ & AS, BDP & & Reservoir & & & Asthma \\
\hline Twisthaler & SP & MF & - & Reservoir & & & Asthma \\
\hline \multicolumn{8}{|c|}{ Third generation: active DPIs, single or multi-dose } \\
\hline Exubera & Pfizer & Insulin & & Blister & & & Diabete \\
\hline Airmax & $\begin{array}{c}\text { Norton } \\
\text { Healthcar } \\
\mathrm{e}\end{array}$ & FO, BUD & & Reservoir & & & $\begin{array}{l}\text { Asthma, } \\
\text { COPD }\end{array}$ \\
\hline \multicolumn{8}{|c|}{ Continued... } \\
\hline
\end{tabular}




\begin{tabular}{|c|c|c|c|c|c|c|c|}
\hline Inhaler & Company & Drug & Carrier & $\begin{array}{c}\text { Powder } \\
\text { supply }\end{array}$ & Dosing & $\begin{array}{c}\text { Fluidization / } \\
\text { Deaggregation }\end{array}$ & Diseases \\
\hline \multicolumn{8}{|c|}{ Next generation DPIs (approved or in development stage) } \\
\hline Aspirair & Vectura & $\begin{array}{l}\text { Apomorphine } \\
\text { hydrochloride }\end{array}$ & - & Powder & Multi & Active & $\begin{array}{c}\text { Erectile } \\
\text { dysfunction }\end{array}$ \\
\hline Omnihaler & $\begin{array}{l}\text { Innoveta } \\
\text { Biomed }\end{array}$ & - & - & - & & Active & \\
\hline Actispire & Britania & Pumactant & & Powder & 1 & Active & - \\
\hline $\begin{array}{l}\text { NEXT }^{\mathrm{TM}} \\
\text { DPI }\end{array}$ & Chiesi & . & - & Reservoir & $\begin{array}{c}\text { Multi- } \\
\text { unit }\end{array}$ & 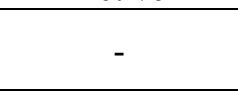 & $\begin{array}{c}\text { Asthma / } \\
\text { COPD }\end{array}$ \\
\hline DirectHaler & Direct-Haler & - & - & Premetered & 1 & - & $\begin{array}{l}\text { Asthma / } \\
\text { COPD }\end{array}$ \\
\hline JAGO & SkyPharma & AS & - & Reservoir & Multi & - & Asthma \\
\hline Turbospin & PH \& T & - & - & Capsule & 1 & - & Asthma \\
\hline AIR & Alkermes & - & - & Capsule & 1 & & - \\
\hline MicroDose & $\mathrm{MDT} / 3 \mathrm{M}$ & $\begin{array}{l}\text { Insulin, } \beta- \\
\text { agonists, } \\
\text { steroids }\end{array}$ & - & blister & $\begin{array}{l}1 \text { or } \\
\text { Multi- } \\
\text { unit }\end{array}$ & $\begin{array}{l}\text { Electronic } \\
\text { /breath }\end{array}$ & $\begin{array}{c}\text { Multi } \\
\text { purpose }\end{array}$ \\
\hline Cyclovent & $\begin{array}{l}\text { Pharma- } \\
\text { chemie }\end{array}$ & Opioids & - & Reservoir & Multi & - & $\begin{array}{l}\text { Dyspnea, } \\
\text { pain }\end{array}$ \\
\hline Dispohaler & AC Pharma & - & - & - & Multi & - & - \\
\hline $\begin{array}{c}\text { CONIX } \\
\text { ONE }\end{array}$ & $\begin{array}{l}\text { Cambridge } \\
\text { Consultant }\end{array}$ & $\begin{array}{c}\text { Vaccines, } \\
\text { anti-asthma }\end{array}$ & - & blister & 1 & - & $\begin{array}{l}\text { Avian flu, } \\
\text { Asthma }\end{array}$ \\
\hline Microhaler & $\begin{array}{l}\text { Harris } \\
\text { Pharm } \\
\end{array}$ & DSCG & - & Capsule & 1 & Passive & Asthma \\
\hline Technohaler & $\begin{array}{l}\text { Innoveta } \\
\text { Biomed }\end{array}$ & - & - & Blister & $\begin{array}{l}\text { Multi- } \\
\text { unit }\end{array}$ & Passive & Asthma \\
\hline Spiros & Dura & AS & Lactose & Blister & $\begin{array}{c}1,16 \\
30\end{array}$ & $\begin{array}{l}\text { Mechan, Turbul. } \\
\text { Shear, impaction }\end{array}$ & Asthma \\
\hline Bulkhaler & Asta Medica & - & - & Reservoir & $\begin{array}{l}\text { Multi- } \\
\text { unit }\end{array}$ & Passive & Asthma \\
\hline Miat-Haler & MiatSpA & $\begin{array}{l}\text { FO, FP, } \\
\text { BUD }\end{array}$ & - & Reservoir & $\begin{array}{l}\text { Multi- } \\
\text { unit }\end{array}$ & Passive & $\begin{array}{l}\text { Asthma, } \\
\text { COPD }\end{array}$ \\
\hline Prohaler & Valois & $\begin{array}{l}\text { TS, SKF- } \\
104353-Q\end{array}$ & Mannitol & Blister & $\begin{array}{l}\text { Multi- } \\
\text { unit }\end{array}$ & $\begin{array}{l}\text { Gas assisted / } \\
\text { turbul., shear }\end{array}$ & Asthma \\
\hline Ostuka DPI & $\begin{array}{l}\text { Otsuka } \\
\text { Pharma }\end{array}$ & 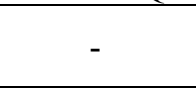 & - & $\begin{array}{c}\text { Compact } \\
\text { cake }\end{array}$ & $\begin{array}{c}\text { Multi- } \\
\text { unit }\end{array}$ & Gas assisted & Asthma \\
\hline Acu-Breath & Respirics & FP & - & Powder & Multi & - & Asthma \\
\hline MF-DPI & - & MF & - & Reservoir & $\begin{array}{c}\text { Multi- } \\
\text { unit }\end{array}$ & - & Asthma \\
\hline Swinhaler & $\begin{array}{l}\text { Otsuka } \\
\text { Pharma }\end{array}$ & $\begin{array}{c}\text { Procaterol, } \\
\text { BUD }\end{array}$ & - & Powder & Multi & - & Asthma \\
\hline $\begin{array}{l}\text { Dynamic Powder } \\
\text { Disperser }\end{array}$ & $\begin{array}{c}\text { Pfeiffer } \\
\text { GmbH }\end{array}$ & - & Lactose & Powder & 12 & Gas assisted & Asthma \\
\hline Certihaler & $\begin{array}{c}\text { Novartis/Sk } \\
\text { ye }\end{array}$ & FO & - & Powder & Multi & Gas assisted & Asthma \\
\hline
\end{tabular}

Source: Adapted from [38, 41, 43, 46, 82, 197].

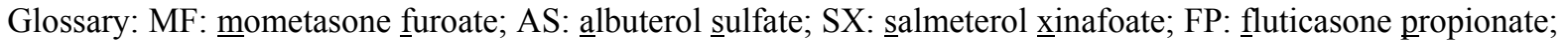
BUD: budesonide; TS: terbutaline sulfate; FE: fenoterol; FO: formoterol; IPB: ipratropium bromide; TI: tiotropium; DSCG: disodium cromoglycate; BDP: beclomethasone dipropionate; EFD: eformoterol fumarate dihydrate; ZM: zanamivir; NI: neuraminidase inhibitors; Lactose: $\alpha$-lactose monohydrate; GSK: GlaxoSmithKline; BI: Boehringer Ingelheim; AZ: ÂstraZeneca; SP: Schering-Plough; MDT: MicroDose Technologies. Dashline (-) denotes information not available. 
Appendix 2-A. TSLI performance data (The order has been sorted for the ease of data analyses; airflow rate $Q=60 \mathrm{~L} / \mathrm{min}$; actuation duration 10s; with solenoid switch; the response data $\left(E D, F P F_{T D}\right.$, and $\left.F P F_{E D}\right)$ mean value, standard deviation in parentheses, replication $n=3$.

\begin{tabular}{|c|c|c|c|c|c|c|}
\hline SETs & Sample & $\begin{array}{c}\text { Blend Conc } \\
(\mathbf{w} / \mathbf{w})\end{array}$ & $\begin{array}{c}\text { Dose } \\
(\boldsymbol{\mu g})\end{array}$ & ED $(\mathbf{\%})$ & $\begin{array}{c}\text { FPF in TD } \\
(\%)\end{array}$ & $\begin{array}{c}\text { FPF in ED } \\
(\%)\end{array}$ \\
\hline A & SV131 & $2.0 \%$ & 505.3 & $90.9(1.2)$ & $25.9(0.9)$ & $28.5(1.2)$ \\
\hline C & SV131 & $2.0 \%$ & 500.7 & $91.6(2.3)$ & $14.3(1.0)$ & $15.6(0.8)$ \\
\hline D & SV131 & $2.0 \%$ & 499.3 & $93.4(0.1)$ & $9.9(1.2)$ & $10.6(1.3)$ \\
\hline A & SV131 & $0.5 \%$ & 252.3 & $89.2(4.3)$ & $17.3(2.7)$ & $19.3(2.1)$ \\
\hline C & SV131 & $0.5 \%$ & 250.2 & $95.0(0.2)$ & $10.5(1.1)$ & $11.1(1.2)$ \\
\hline D & SV131 & $0.5 \%$ & 250.7 & $91.1(2.6)$ & $10.0(1.5)$ & $11.0(2.0)$ \\
\hline A & ML058 & $2.0 \%$ & 501.3 & $74.0(4.7)$ & $35.2(2.7)$ & $47.6(2.7)$ \\
\hline C & ML058 & $2.0 \%$ & 500.0 & $87.2(1.0)$ & $30.4(2.5)$ & $34.8(3.0)$ \\
\hline D & ML058 & $2.0 \%$ & 501.3 & $88.4(1.4)$ & $22.4(4.2)$ & $25.4(4.7)$ \\
\hline A & ML058 & $0.5 \%$ & 251.4 & $76.6(4.1)$ & $33.7(2.6)$ & $43.9(1.3)$ \\
\hline C & ML058 & $0.5 \%$ & 250.3 & $88.6(2.3)$ & $24.4(5.3)$ & $27.5(5.3)$ \\
\hline D & ML058 & $0.5 \%$ & 251.3 & $90.9(1.5)$ & $23.7(3.5)$ & $26.0(3.5)$ \\
\hline
\end{tabular}




\section{Appendix 2-B}

Experimental design of albuterol sulfate - lactose monohydrate blends for performance study:

Factors: Lactose (SV473, ML 780); concentration (0.5\%, 2\%); SETs (D, A)

Response: $\mathrm{ED}, \mathrm{FPF}_{\mathrm{TD}}, \mathrm{FPF}_{\mathrm{ED}}$

$\begin{array}{lcccccc}\text { Run } & \begin{array}{c}\text { Factor B: } \\ \text { Lactose }\end{array} & \begin{array}{c}\text { Factor C: } \\ \text { Tube }\end{array} & \begin{array}{c}\text { Factor D } \\ \text { Conc } \%\end{array} & \begin{array}{c}\text { Response } \\ \text { ED \% }\end{array} & \begin{array}{c}\text { Response } \\ \text { FPF in TD\% }\end{array} & \begin{array}{c}\text { Response } \\ \text { FPF in ED\% }\end{array} \\ 1 & \text { SV473 } & \text { D } & 2 & 91.8 & 8.9 & 9.7 \\ 2 & \text { ML780 } & \text { D } & 2 & 84.7 & 23.0 & 27.1 \\ 3 & \text { SV473 } & \text { A } & 2 & 77.8 & 23.4 & 30.1 \\ 4 & \text { SV473 } & \text { D } & 0.5 & 84.8 & 8.9 & 10.5 \\ 5 & \text { SV473 } & \text { A } & 0.5 & 87.8 & 17.6 & 20.0 \\ 6 & \text { ML780 } & \text { D } & 0.5 & 87.5 & 18.3 & 21.0 \\ 7 & \text { ML780 } & \text { A } & 2 & 81.2 & 45.4 & 56.0 \\ 8 & \text { ML780 } & \text { A } & 0.5 & 80.8 & 34.8 & 43.1\end{array}$

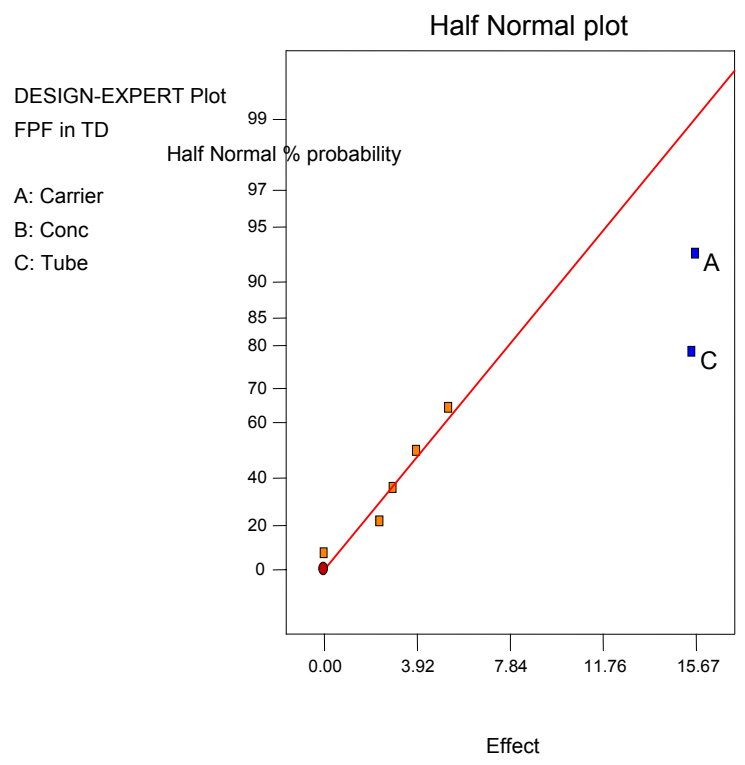




\section{Appendix 2-C}

Experimental design of albuterol sulfate - lactose monohydrate blends for performance study:

Factors: Lactose (SV131, ML058); concentration (0.5\%, 2\%); SETs (D, A)

Response: $\mathrm{ED}, \mathrm{FPF}_{\mathrm{TD}}, \mathrm{FPF}_{\mathrm{ED}}$

$\begin{array}{ccccccc}\text { Run } & \begin{array}{c}\text { Factor B: } \\ \text { Lactose }\end{array} & \begin{array}{c}\text { Factor C: } \\ \text { Tube }\end{array} & \begin{array}{c}\text { Factor D } \\ \text { Conc } \%\end{array} & \begin{array}{c}\text { Response } \\ \text { ED \% }\end{array} & \begin{array}{c}\text { Response } \\ \text { FPF in TD\% }\end{array} & \begin{array}{c}\text { Response } \\ \text { FPF in ED\% }\end{array} \\ 1 & \text { SV131 } & \text { D } & 0.5 & 91.1 & 10.0 & 11.0 \\ 2 & \text { ML058 } & \text { A } & 0.5 & 76.6 & 33.7 & 43.9 \\ 3 & \text { SV131 } & \text { D } & 2 & 93.4 & 9.9 & 10.6 \\ 4 & \text { SV131 } & \text { A } & 2 & 90.9 & 25.9 & 28.5 \\ 5 & \text { ML058 } & \text { D } & 2 & 88.4 & 22.4 & 25.4 \\ 6 & \text { SV131 } & \text { A } & 0.5 & 89.2 & 17.3 & 19.3 \\ 7 & \text { ML058 } & \text { A } & 2 & 74.0 & 35.2 & 47.6 \\ 8 & \text { ML058 } & \text { D } & 0.5 & 90.9 & 23.7 & 26.0\end{array}$

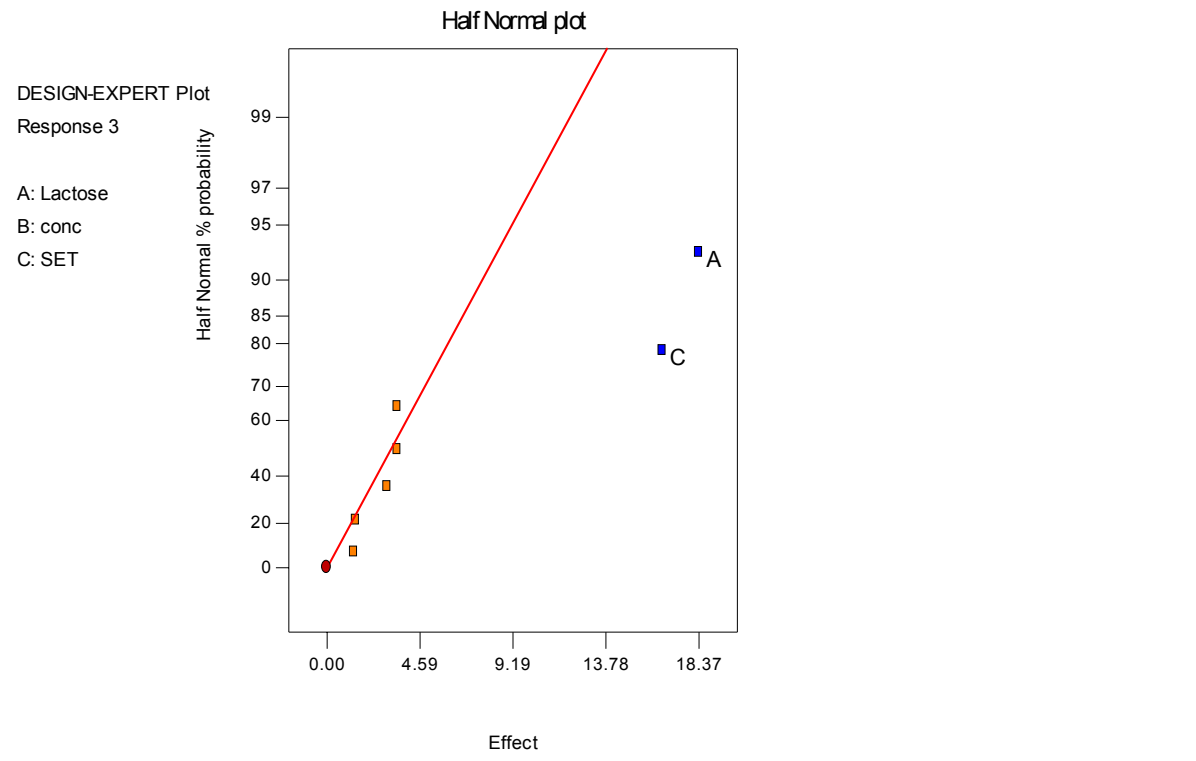

Summary of finding from Appendix-B and C: The notable contributing variables for the $\mathrm{FPF}_{\mathrm{TD}}$ and $\mathrm{FPF}_{\mathrm{ED}}$ were lactose and applied shear (SETs), while the drug concentration contribution was not statistically significant. The contributing variables for the ED were not significant. 
APPENDIX 3-A1. - TSLI performance data of albuterol sulfate (AS) and disodium cromoglycate (DSCG) (The order has been sorted for the ease of data analyses; drug conc $2 \%(\mathrm{w} / \mathrm{w})$; airflow rate $\mathrm{Q}=60 \mathrm{~L} / \mathrm{min}$; actuation duration $10 \mathrm{~s}$; with solenoid switch)

\begin{tabular}{|c|c|c|c|c|c|c|c|c|c|c|c|c|c|}
\hline Drug & Carrier & SET & $\begin{array}{l}\text { ED } \\
(\%)\end{array}$ & $\begin{array}{c}\text { FPFTD } \\
(\%)\end{array}$ & $\begin{array}{c}\text { FPFED } \\
(\%)\end{array}$ & $\begin{array}{l}\text { FPM } \\
(\mu \mathrm{g})\end{array}$ & Drug & Carrier & SET & $\begin{array}{l}\text { ED } \\
(\%)\end{array}$ & $\begin{array}{c}\text { FPFTD } \\
(\%)\end{array}$ & $\begin{array}{c}\text { FPFED } \\
(\%)\end{array}$ & $\begin{array}{l}\text { FPM } \\
(\mu \mathrm{g})\end{array}$ \\
\hline AS & SV208 & $A$ & 77.02 & 14.22 & 18.47 & 68.91 & DSCG & SV208 & $A$ & 75.40 & 38.26 & 50.74 & 185.42 \\
\hline AS & SV208 & $A$ & 87.88 & 16.12 & 18.34 & 74.43 & DSCG & SV208 & A & 75.69 & 33.52 & 44.29 & 157.53 \\
\hline AS & SV208 & $A$ & 83.67 & 13.89 & 16.60 & 69.73 & DSCG & SV208 & A & 73.32 & 28.88 & 39.39 & 146.63 \\
\hline AS & V208 & $B$ & 81.02 & 9.43 & 11.64 & 46.13 & DSCG & SV208 & B & 81.18 & 33.19 & 40.88 & 159.48 \\
\hline AS & SV208 & $B$ & 84.01 & 11.89 & 14.15 & 57.05 & DSCG & SV208 & B & 83.63 & 35.02 & 41.87 & 183.22 \\
\hline AS & V208 & $B$ & 86.64 & 13.16 & 15.19 & 71.59 & DSCG & SV208 & B & 77.76 & 29.70 & 8.19 & 143.92 \\
\hline AS & SV208 & C & 92.09 & 9.13 & 9.91 & 44.66 & DSCG & SV208 & C & 91.01 & 33.97 & 7.33 & 161.39 \\
\hline AS & SV208 & C & 93.54 & 8.71 & 9.31 & 41.02 & DSCG & SV208 & C & 90.98 & 34.08 & 7.46 & 167.36 \\
\hline AS & SV208 & C & 91.04 & 11.85 & 13.02 & 57.74 & DSCG & SV208 & C & 87.94 & 32.11 & 36.52 & 158.53 \\
\hline AS & SV208 & D & 87.98 & 8.92 & 10.14 & 45.47 & DSCG & SV208 & D & 94.75 & 22.80 & 24.07 & 111.99 \\
\hline AS & & D & 93.38 & 6.65 & 7.13 & 31.36 & DSCG & & D & 92.72 & 22.83 & 24.62 & 108.67 \\
\hline AS & & D & 88.80 & & & 35.23 & DSCG & & D & 86.33 & .89 & 4.19 & 4.76 \\
\hline AS & 473 & A & 77.80 & 23.39 & 30.06 & 114.70 & DSCG & 58 & $A$ & 71.48 & 39.58 & 5.37 & 9.54 \\
\hline AS & 473 & A & 91.05 & 13.65 & 14.99 & 66.70 & DSCG & 58 & A & 80.47 & 48.01 & 9.66 & 5.75 \\
\hline AS & V473 & A & 88.83 & 12.64 & 14.23 & 68.75 & DSCG & 58 & $A$ & 79.54 & 42.42 & 3.33 & 211.19 \\
\hline AS & SV473 & $B$ & 88.60 & 9.80 & 11.06 & 47.48 & DSCG & & B & 69.82 & 38.54 & 55.20 & 186.56 \\
\hline AS & SV473 & $B$ & 93.50 & 9.14 & 9.78 & 45.11 & DSCG & $\mathrm{ML}$ & B & 71.29 & 35.95 & 50.42 & 175.88 \\
\hline AS & SV473 & $B$ & 90.33 & 12.47 & 13.81 & 67.27 & DSCG & & B & 70.40 & 36.95 & 52.48 & 186.98 \\
\hline AS & & $C$ & 93.8 & & & 54.02 & & & $C$ & 85.74 & & .10 & \\
\hline AS & SV473 & $C$ & 95.44 & & & 42.73 & $\overline{S C G}$ & & $c$ & 83.75 & & .00 & \\
\hline AS & 473 & $C$ & 95.59 & 10.26 & 10.74 & 52.39 & $S C G$ & & $c$ & 83.79 & & 9.76 & \\
\hline AS & 473 & D & 91.84 & & & 44.21 & SCG & & D & 6.76 & 6.87 & 0.98 & 134.02 \\
\hline AS & SV473 & D & 96.25 & & & 28.18 & DSCG & 58 & D & 80.97 & 18.40 & 2.73 & 89.22 \\
\hline AS & SV473 & D & 91.36 & 5.87 & 6.43 & 30.34 & DSCG & $\mathrm{MLC}$ & D & 84.62 & 17.35 & 20.51 & 88.99 \\
\hline AS & ML058 & A & 81.16 & 45.43 & 55.98 & 236.28 & DSCG & SV473 & A & 79.00 & 43.63 & 55.23 & 214.12 \\
\hline AS & & $A$ & 85.64 & & & 210.57 & & & $A$ & 75.51 & & 1.08 & 143.62 \\
\hline AS & & $A$ & 83.82 & & & 220.68 & SCG & & A & 75.42 & 32.57 & 3.19 & \\
\hline AS & $\mathrm{N}$ & $B$ & 77.23 & 36.19 & 86 & 177.41 & DSCG & & $B$ & 83.36 & 39.08 & 0.87 & \\
\hline AS & $\bar{M}$ & $B$ & 73.64 & 35.24 & & 177.95 & DSCG & & $B$ & 81.40 & 34.81 & 2.76 & 162.52 \\
\hline AS & L058 & $B$ & 76.59 & 36.40 & & 191.28 & DSCG & SV473 & $B$ & 79.53 & 30.12 & 7.88 & 149.88 \\
\hline AS & L058 & $C$ & 88.30 & 31.43 & & 160.26 & DSCG & SV473 & $C$ & 91.89 & 33.70 & 36.67 & 154.83 \\
\hline AS & ML058 & $C$ & 87.14 & 31.03 & 35.61 & 160.00 & DSCG & SV473 & $C$ & 90.85 & 36.99 & 40.72 & 169.74 \\
\hline AS & ML058 & $C$ & 84.99 & 28.60 & 33.66 & 142.44 & DSCG & SV473 & $C$ & 89.43 & 36.14 & 40.41 & 174.04 \\
\hline AS & & $D$ & 84.66 & 22.96 & & 114.70 & DSCG & & $\mathrm{D}$ & 92.43 & 27.12 & 9.34 & 129.07 \\
\hline AS & & $D$ & 80.74 & 17.70 & 21.92 & 88.07 & DSCG & SV473 & $\mathrm{D}$ & 91.17 & 25.78 & 8.28 & 120.30 \\
\hline AS & $\mathrm{N}$ & $\mathrm{D}$ & 84.86 & 23.53 & 27.72 & 127.05 & DSCG & SV473 & $\mathrm{D}$ & 88.45 & 23.18 & 6.21 & 1.99 \\
\hline AS & L780 & A & 80.22 & 27.39 & 34. & 151.76 & DSCG & ML780 & $A$ & 82.61 & 47.26 & 7.21 & 231.87 \\
\hline AS & L780 & $A$ & 82.79 & 38.30 & 46.26 & 191.70 & DSCG & ML780 & A & 81.35 & 50.00 & 61.46 & 232.35 \\
\hline AS & ML780 & $A$ & 76.32 & 36.31 & 47.57 & 194.66 & DSCG & ML780 & A & 79.00 & 41.66 & 52.74 & 209.40 \\
\hline AS & ML780 & $B$ & 72.39 & 28.53 & 39.42 & 142.22 & DSCG & ML780 & $B$ & 68.76 & 42.51 & 61.82 & 204.21 \\
\hline AS & ML780 & $B$ & 69.62 & 35.47 & 50.95 & 182.50 & DSCG & ML780 & $B$ & 67.11 & 39.37 & 58.66 & 187.04 \\
\hline AS & ML780 & $B$ & 79.50 & 35.10 & & 200.23 & DSCG & & $B$ & 72.73 & 38.93 & 53.53 & 194.73 \\
\hline AS & 1700 & $C$ & 81.52 & 17.50 & & 83.57 & DSCG & ML780 & $C$ & 85.31 & 39.23 & 5.99 & 189.42 \\
\hline AS & & $C$ & 87.49 & & & 98.75 & DSCG & & $C$ & 82.57 & 34.30 & 1.54 & 163.72 \\
\hline AS & 1780 & C & 85.43 & 26.25 & 30.73 & 135.23 & DSCG & ML780 & C & 83.13 & 37.05 & 4.57 & 185.55 \\
\hline AS & & $\mathrm{D}$ & & & & 74.88 & DSCG & & $\mathrm{D}$ & 85.46 & 23.58 & 27.60 & 112.65 \\
\hline AS & L780 & $D$ & 86.46 & 16.26 & 18.80 & 81.25 & DSCG & ML780 & $D$ & 88.70 & 23.82 & 26.85 & 116.69 \\
\hline AS & ML780 & $\mathrm{D}$ & 76.52 & 11.14 & 14.56 & 58.48 & DSCG & ML780 & $\mathrm{D}$ & 83.92 & 17.51 & 20.87 & 90.12 \\
\hline
\end{tabular}


APPENDIX 3-A2. - TSLI performance data of ipratropium bromide monohydrate (IPB) and fluticasone propionate (FP) (The order has been sorted for the ease of data analyses; drug conc $2 \%(\mathrm{w} / \mathrm{w})$; airflow rate $\mathrm{Q}=60 \mathrm{~L} / \mathrm{min}$; actuation duration $10 \mathrm{~s}$; with solenoid switch)

\begin{tabular}{|c|c|c|c|c|c|c|c|c|c|c|c|c|c|}
\hline Drug & Carrier & SET & $\begin{array}{l}\text { ED } \\
(\%)\end{array}$ & $\begin{array}{c}\text { FPFTD } \\
(\%)\end{array}$ & $\begin{array}{l}\text { FPFED } \\
\text { (\%) }\end{array}$ & $\begin{array}{l}\text { FPM } \\
(\mu \mathrm{g})\end{array}$ & Drug & Carrier & SET & $\begin{array}{l}\text { ED } \\
(\%)\end{array}$ & $\begin{array}{c}\text { FPFTD } \\
(\%)\end{array}$ & $\begin{array}{l}\text { FPFED } \\
\text { (\%) }\end{array}$ & $\begin{array}{l}\text { FPM } \\
(\mu \mathrm{g})\end{array}$ \\
\hline IPB & SV208 & A & 71.95 & 33.06 & 45.95 & 173.38 & FP & SV208 & A & 72.21 & 15.64 & 21.65 & 78.03 \\
\hline IPB & SV208 & A & 69.57 & 29.12 & 41.85 & 148.92 & FP & SV208 & $A$ & 68.70 & 14.98 & 21.80 & 78.42 \\
\hline IPB & SV208 & A & 71.25 & 32.91 & 46.19 & 170.62 & FP & SV208 & A & 69.07 & 14.75 & 21.36 & 78.34 \\
\hline IPB & SV208 & $B$ & 72.84 & 31.96 & 43.87 & 151.23 & FP & SV208 & $B$ & 68.72 & 13.47 & 19.59 & 65.05 \\
\hline IPB & SV208 & $B$ & 71.54 & 32.77 & 45.81 & 158.46 & $\mathrm{FP}$ & SV208 & $\mathrm{B}$ & 68.79 & 13.53 & 19.67 & 65.83 \\
\hline IPB & SV208 & $B$ & 71.83 & 33.60 & 46.77 & 157.69 & $\mathrm{FP}$ & SV208 & $B$ & 68.80 & 13.56 & 19.71 & 66.61 \\
\hline IPB & SV208 & $\mathrm{C}$ & 87.06 & 31.91 & 36.65 & 145.38 & $\mathrm{FP}$ & SV208 & $\mathrm{C}$ & 84.37 & 15.86 & 18.79 & 80.83 \\
\hline IPB & SV208 & C & 88.07 & 36.14 & 41.04 & 157.23 & FP & SV208 & C & 88.05 & 15.12 & 7.17 & 79.97 \\
\hline IPB & SV208 & C & 88.63 & 33.63 & 37.94 & 160.15 & FP & SV208 & $C$ & 89.52 & 16.23 & 18.13 & 81.76 \\
\hline IPB & SV208 & $\mathrm{D}$ & 91.04 & 29.48 & 32.38 & 161.23 & $\mathrm{FP}$ & SV208 & $\mathrm{D}$ & 86.90 & 10.55 & 12.14 & 50.69 \\
\hline IPB & SV208 & $\mathrm{D}$ & 89.07 & 32.23 & 36.19 & 159.54 & $\mathrm{FP}$ & SV208 & D & 88.08 & 14.41 & 16.36 & 74.61 \\
\hline IPB & V208 & $\mathrm{D}$ & 91.83 & 30.20 & 2.89 & 137.38 & FP & SV208 & $\mathrm{D}$ & 89.23 & 5.00 & 6.81 & 81.68 \\
\hline IPB & 1473 & A & 68.09 & 33.94 & .84 & 161.38 & $\mathrm{FP}$ & SV473 & A & 69.66 & 7.09 & 4.53 & 87.28 \\
\hline IPB & SV473 & A & 67.22 & 31.26 & .51 & 146.15 & FP & SV473 & A & 70.91 & 6.27 & 2.94 & 82.23 \\
\hline IPB & SV473 & A & 71.40 & 28.81 & 40.35 & 150.15 & $\mathrm{FP}$ & SV473 & A & 69.97 & 3.66 & 9.52 & 73.45 \\
\hline IPB & SV473 & B & 73.26 & 24.74 & 3.77 & 132.92 & FP & SV473 & $B$ & 70.59 & 14.30 & 0.26 & 71.74 \\
\hline IPB & SV473 & $B$ & 72.71 & 29.34 & 40.36 & 136.15 & FP & SV473 & $B$ & 73.72 & 16.01 & 21.72 & 82.38 \\
\hline IPB & SV473 & B & 67.05 & 23.51 & 35.07 & 120.31 & FP & SV473 & B & 70.91 & 13.99 & 19.73 & 69.87 \\
\hline IPB & SV473 & C & 83.46 & & 43.46 & 193.38 & FP & SV473 & C & 86.45 & 14.88 & 17.21 & 79.04 \\
\hline IPB & & $C$ & 81.02 & & & & FP & & $C$ & 6.77 & 14.80 & .06 & 73.06 \\
\hline IPB & SV473 & $C$ & 86.59 & 33.20 & 34 & & $\mathrm{FP}$ & SV473 & $C$ & 85.94 & 13.63 & 86 & 68.91 \\
\hline IPB & SV473 & $\mathrm{D}$ & 87.76 & 27.46 & .29 & 144.00 & $\mathrm{FP}$ & SV473 & $\mathrm{D}$ & 90.04 & 12.16 & 3.51 & 55.73 \\
\hline IPB & 473 & L & 90.32 & & & & FP & 473 & $\bar{D}$ & 1.48 & 11.37 & 2.43 & 51.37 \\
\hline IPB & V473 & $\mathrm{D}$ & 90.69 & 28.91 & 1.87 & 143.38 & FP & SV473 & $\mathrm{D}$ & 0.03 & 8.86 & 9.85 & 41.35 \\
\hline IPB & L058 & $\mathrm{A}$ & 72.17 & 37.74 & 52.29 & 190.31 & $\mathrm{FP}$ & ML058 & A & 67.12 & 20.84 & 31.05 & 112.38 \\
\hline IPB & L058 & A & 69.50 & 31.62 & 45.50 & 164.92 & FP & ML058 & A & 65.62 & 21.28 & 32.43 & 111.68 \\
\hline IPB & L058 & $A$ & 71.97 & 28.00 & 38.90 & 149.38 & FP & ML058 & $A$ & 64.22 & 18.90 & 9.44 & 100.80 \\
\hline IPB & & B & 70.00 & & & 147.85 & FP & & $B$ & 50.75 & 20.29 & 99 & 94.21 \\
\hline IPB & ML058 & $\mathrm{B}$ & 63.54 & 37.05 & 31 & 179.08 & FP & 058 & B & 52.51 & 16.01 & 0.49 & 74.81 \\
\hline IPB & $\mathrm{M}$ & B & 66.81 & & 4.86 & 187.38 & $\mathrm{FP}$ & & $B$ & 51.19 & 20.09 & 9.25 & 86.66 \\
\hline IPB & & C & 83.84 & & & & FP & & C & 2.88 & 3.97 & 6.85 & 69.02 \\
\hline IPB & ML058 & $C$ & 77.60 & 29.63 & 38.18 & 176.00 & FP & ML058 & C & 79.87 & 11.46 & 14.35 & 59.66 \\
\hline IPB & ML058 & C & 80.31 & 29.98 & 37.33 & 155.85 & FP & ML058 & $C$ & 83.24 & 12.53 & 15.05 & 67.54 \\
\hline IPB & ML058 & $\mathrm{D}$ & 85.39 & 21.44 & 25.11 & 116.15 & FP & ML058 & $\mathrm{D}$ & 82.08 & 15.94 & 19.42 & 74.53 \\
\hline IPB & & $\mathrm{D}$ & 88.31 & & & & FP & & $D$ & 88.20 & 13.78 & 5.62 & 66.84 \\
\hline IPB & & $D$ & 89.99 & & & & FP & & $\mathrm{D}$ & 81.73 & 17.00 & 0.79 & 72.98 \\
\hline IPB & $\mathrm{M}$ & A & 66.89 & & 4 & & $\mathrm{FP}$ & ML780 & A & 70.90 & 25.76 & 6.34 & 131.89 \\
\hline IPB & $\mathrm{N}$ & A & 70.17 & & & 196.62 & $\mathrm{FP}$ & ML780 & $\mathrm{A}$ & 68.83 & 25.46 & 6.98 & 134.38 \\
\hline IPB & ML780 & A & 70.02 & & 49.89 & 181.38 & FP & ML780 & $A$ & 65.53 & 21.48 & 32.77 & 112.54 \\
\hline IPB & ML780 & $B$ & 67.12 & 31.56 & 47.02 & 167.85 & $\mathrm{FP}$ & ML780 & $B$ & 58.40 & 22.70 & 38.87 & 109.81 \\
\hline IPB & ML780 & $B$ & 70.04 & 38.25 & 54.61 & 188.92 & $\mathrm{FP}$ & ML780 & B & 62.27 & 25.95 & 41.67 & 135.16 \\
\hline IPB & ML780 & B & 63.66 & 38.58 & 60.60 & 182.31 & FP & ML780 & $B$ & 60.19 & 25.19 & 41.85 & 123.73 \\
\hline IPB & ML780 & $C$ & 84.59 & 26.85 & 31.74 & 124.92 & FP & ML780 & $C$ & 78.74 & 20.08 & 25.50 & 107.88 \\
\hline IPB & ML780 & C & 84.13 & 27.18 & 32.30 & 139.08 & FP & ML780 & C & 79.26 & 19.76 & 24.93 & 107.41 \\
\hline IPB & ML780 & C & 79.05 & 28.86 & & 129.08 & $\mathrm{FP}$ & ML780 & C & 84.69 & 27.09 & 1.98 & 146.58 \\
\hline IPB & ML780 & $\mathrm{D}$ & 88.47 & 21.07 & & 102.46 & FP & ML780 & D & 88.20 & 21.46 & 4.34 & 104.38 \\
\hline IPB & & D & 82.19 & & & & $\mathrm{FP}$ & & $\mathrm{D}$ & 87.48 & 22.14 & 25.31 & 110.91 \\
\hline IPB & ML780 & D & 83.46 & 23.37 & 28.00 & 129.23 & FP & ML780 & $D$ & 82.89 & 22.35 & 26.96 & 108.11 \\
\hline
\end{tabular}


Appendix. 4-A. TSLI Performance Data Using SET (2\% (w/w) drug conc., $10 \mathrm{~s}$ actuation)

\begin{tabular}{|c|c|c|c|c|c|}
\hline Drug & Lactose & Tube & ED (\%) & FPF $_{\mathrm{TD}}(\%)$ & $\operatorname{FPF}_{E D}(\%)$ \\
\hline AS & Treh & A & 86.0 & 13.9 & 16.1 \\
\hline AS & Treh & $A$ & 93.0 & 12.1 & 13.0 \\
\hline AS & Treh & $A$ & 91.7 & 12.5 & 13.6 \\
\hline AS & Treh & B & 94.5 & 9.0 & 9.6 \\
\hline AS & Treh & $B$ & 94.2 & 8.4 & 8.9 \\
\hline AS & Treh & B & 92.4 & 11.2 & 12.1 \\
\hline AS & Treh & C & 95.4 & 7.4 & 7.8 \\
\hline AS & Treh & $C$ & 95.1 & 6.4 & 6.7 \\
\hline AS & Treh & C & 95.8 & 9.4 & 9.8 \\
\hline AS & Treh & $\mathrm{D}$ & 95.3 & 6.3 & 6.6 \\
\hline AS & Treh & $\mathrm{D}$ & 89.9 & 4.1 & 4.5 \\
\hline AS & Treh & $\mathrm{D}$ & 94.0 & 5.7 & 6.1 \\
\hline AS & Mann & A & 88.0 & 12.2 & 13.8 \\
\hline AS & Mann & $A$ & 94.1 & 10.2 & 10.8 \\
\hline AS & Mann & $A$ & 89.9 & 9.9 & 11.0 \\
\hline AS & Mann & B & 93.9 & 9.3 & 10.0 \\
\hline AS & Mann & $B$ & 94.3 & 6.6 & 7.0 \\
\hline AS & Mann & $B$ & 91.2 & 7.4 & 8.1 \\
\hline AS & Mann & C & 94.8 & 8.8 & 9.3 \\
\hline AS & Mann & $C$ & 95.7 & 5.7 & 6.0 \\
\hline AS & Mann & $C$ & 94.0 & 8.0 & 8.5 \\
\hline AS & Mann & D & 90.1 & 5.0 & 5.6 \\
\hline AS & Mann & $\mathrm{D}$ & 97.3 & 5.2 & 5.4 \\
\hline AS & Mann & $\mathrm{D}$ & 93.0 & 5.2 & 5.6 \\
\hline DSCG & Treh & A & 86.9 & 22.1 & 25.4 \\
\hline DSCG & Treh & $A$ & 85.6 & 15.3 & 17.9 \\
\hline DSCG & Treh & A & 83.2 & 11.8 & 14.2 \\
\hline DSCG & Treh & B & 88.4 & 17.4 & 19.7 \\
\hline DSCG & Treh & B & 95.2 & 17.3 & 18.2 \\
\hline DSCG & Treh & B & 86.4 & 17.5 & 20.3 \\
\hline DSCG & Treh & C & 94.2 & 16.8 & 17.8 \\
\hline DSCG & Treh & $C$ & 94.4 & 16.6 & 17.6 \\
\hline DSCG & Treh & $C$ & 95.4 & 16.5 & 17.3 \\
\hline DSCG & Treh & D & 95.4 & 11.2 & 11.7 \\
\hline DSCG & Treh & $\mathrm{D}$ & 97.2 & 11.4 & 11.7 \\
\hline DSCG & Treh & $\mathrm{D}$ & 93.9 & 10.4 & 11.1 \\
\hline DSCG & Mann & A & 88.1 & 27.0 & 30.7 \\
\hline DSCG & Mann & A & 86.8 & 20.4 & 23.5 \\
\hline DSCG & Mann & A & 85.0 & 19.0 & 22.4 \\
\hline DSCG & Mann & B & 90.0 & 17.1 & 19.0 \\
\hline DSCG & Mann & $B$ & 88.6 & 17.6 & 19.9 \\
\hline DSCG & Mann & B & 86.3 & 15.4 & 17.8 \\
\hline DSCG & Mann & C & 93.7 & 15.9 & 17.0 \\
\hline DSCG & Mann & $C$ & 92.5 & 18.2 & 19.7 \\
\hline DSCG & Mann & $C$ & 89.8 & 14.0 & 15.5 \\
\hline DSCG & Mann & D & 94.7 & 12.9 & 13.6 \\
\hline DSCG & Mann & $\mathrm{D}$ & 90.0 & 7.4 & 8.2 \\
\hline DSCG & Mann & $\mathrm{D}$ & 86.5 & 9.4 & 10.9 \\
\hline & & & & & \\
\hline
\end{tabular}




\begin{tabular}{|c|c|c|c|c|c|}
\hline Drug & Lactose & Tube & ED (\%) & $\mathrm{FPF}_{\mathrm{TD}}(\%)$ & FPF $_{\text {ED }}(\%)$ \\
\hline IPR & Treh & A & 76.7 & 12.3 & 16.1 \\
\hline IPR & Treh & $A$ & 80.5 & 16.5 & 20.5 \\
\hline IPR & Treh & $\bar{A}$ & 81.0 & 16.3 & 20.2 \\
\hline IPR & Treh & $B$ & 80.8 & 16.3 & 20.1 \\
\hline IPR & Treh & $B$ & 84.3 & 15.5 & 18.4 \\
\hline IPR & Treh & $B$ & 83.8 & 14.7 & 17.6 \\
\hline IPR & Treh & C & 88.0 & 9.8 & 11.1 \\
\hline IPR & Treh & C & 92.0 & 12.1 & 13.1 \\
\hline IPR & Treh & C & 91.6 & 10.3 & 11.3 \\
\hline IPR & Treh & D & 90.8 & 4.2 & 4.6 \\
\hline IPR & Treh & D & 93.3 & 5.0 & 5.4 \\
\hline IPR & Treh & $\mathrm{D}$ & 93.7 & 6.4 & 6.9 \\
\hline IPR & Mann & A & 75.0 & 8.3 & 11.1 \\
\hline IPR & Mann & A & 78.4 & 9.5 & 12.1 \\
\hline IPR & Mann & $A$ & 72.9 & 9.5 & 13.0 \\
\hline IPR & Mann & $B$ & 77.7 & 12.6 & 16.2 \\
\hline IPR & Mann & $B$ & 74.3 & 11.4 & 15.3 \\
\hline IPR & Mann & $B$ & 79.0 & 10.2 & 12.9 \\
\hline IPR & Mann & C & 92.3 & 10.0 & 10.3 \\
\hline IPR & Mann & $C$ & 90.4 & 8.7 & 9.7 \\
\hline IPR & Mann & $C$ & 86.7 & 5.3 & 6.1 \\
\hline IPR & Mann & D & 84.8 & 3.2 & 3.8 \\
\hline IPR & Mann & $\mathrm{D}$ & 74.6 & 1.8 & 2.4 \\
\hline IPR & Mann & $\mathrm{D}$ & 72.9 & 3.7 & 5.1 \\
\hline FLUT & Treh & $A$ & 64.9 & 7.9 & 12.1 \\
\hline FLUT & Treh & $A$ & 67.3 & 8.1 & 12.1 \\
\hline FLUT & Treh & $A$ & 65.7 & 11.0 & 16.7 \\
\hline FLUT & Treh & $B$ & 66.6 & 4.9 & 7.3 \\
\hline FLUT & Treh & $B$ & 64.7 & 5.4 & 8.3 \\
\hline FLUT & Treh & $B$ & 65.8 & 5.2 & 7.9 \\
\hline FLUT & Treh & C & 87.6 & 8.3 & 9.4 \\
\hline FLUT & Treh & C & 86.2 & 11.4 & 13.2 \\
\hline FLUT & Treh & C & 86.9 & 8.8 & 10.2 \\
\hline FLUT & Treh & D & 91.7 & 7.1 & 7.7 \\
\hline FLUT & Treh & D & 88.4 & 6.5 & 7.4 \\
\hline FLUT & Treh & D & 90.6 & 6.7 & 7.4 \\
\hline FLUT & Mann & $A$ & 67.5 & 11.8 & 17.5 \\
\hline FLUT & Mann & $A$ & 68.9 & 11.6 & 16.8 \\
\hline FLUT & Mann & $A$ & 71.8 & 14.6 & 20.3 \\
\hline FLUT & Mann & B & 73.6 & 15.2 & 20.7 \\
\hline FLUT & Mann & $B$ & 77.4 & 13.9 & 17.9 \\
\hline FLUT & Mann & $B$ & 76.2 & 14.6 & 19.1 \\
\hline FLUT & Mann & C & 90.5 & 13.9 & 15.4 \\
\hline FLUT & Mann & C & 84.4 & 14.7 & 17.4 \\
\hline FLUT & Mann & C & 86.9 & 14.4 & 16.5 \\
\hline FLUT & Mann & D & 89.7 & 9.7 & 10.8 \\
\hline FLUT & Mann & D & 91.6 & 9.8 & 10.7 \\
\hline FLUT & Mann & D & 90.6 & 10.2 & 11.2 \\
\hline
\end{tabular}


Appendix 5-A. The Schematic Calculation of Aerodynamic Parameters of Extra SETs.

\begin{tabular}{|c|c|c|c|c|}
\hline $\begin{array}{l}\text { Aerosol } \\
\text { Device }\end{array}$ & $\begin{array}{c}\text { Reynold's No., } \\
\text { Re, }\end{array}$ & $\begin{array}{c}\text { Shear Stress } \\
\tau_{\mathrm{s}} \\
\left(\mathrm{N} / \mathrm{m}^{2}\right)\end{array}$ & $\begin{array}{l}\text { Power } \\
\text { (Nm/s) }\end{array}$ & $\begin{array}{c}\text { Pressure drop, } \\
\Delta P\left(\mathrm{~N} / \mathrm{m}^{2}\right)\end{array}$ \\
\hline SET A & 18443 & 13.143 & 6.9194 & 6919.38 \\
\hline SET A1 & 16196 & 8.742 & & \\
\hline SET B & 13440 & 4.342 & 1.4915 & 1491.55 \\
\hline SET B1 & 12583 & 3.270 & & \\
\hline SET C & 11066 & 2.199 & 0.7470 & 747.01 \\
\hline SET C1 & 10126 & 1.412 & & \\
\hline SET D & 7720 & 0.624 & 0.1557 & 155.69 \\
\hline Inhalator & 25865 & 42.934 & 5.3410 & 5340.98 \\
\hline Rotahaler & 10810 & 2.026 & 0.4575 & 457.53 \\
\hline Aerolizer & 8881 & 8.248 & 1.8500 & 1850.00 \\
\hline
\end{tabular}

1) The SET parameter

New SET A1, B1, C1 have mean shear stress between SET A and B, SET B and C, and SET C and $\mathrm{D}$, respectively, which are $1.412,3.270,8.742 \mathrm{~N} / \mathrm{m}^{2}$.

2) The shear stress determined by $\mathrm{Re}-\tau^{1 / 4}$ linear regression.

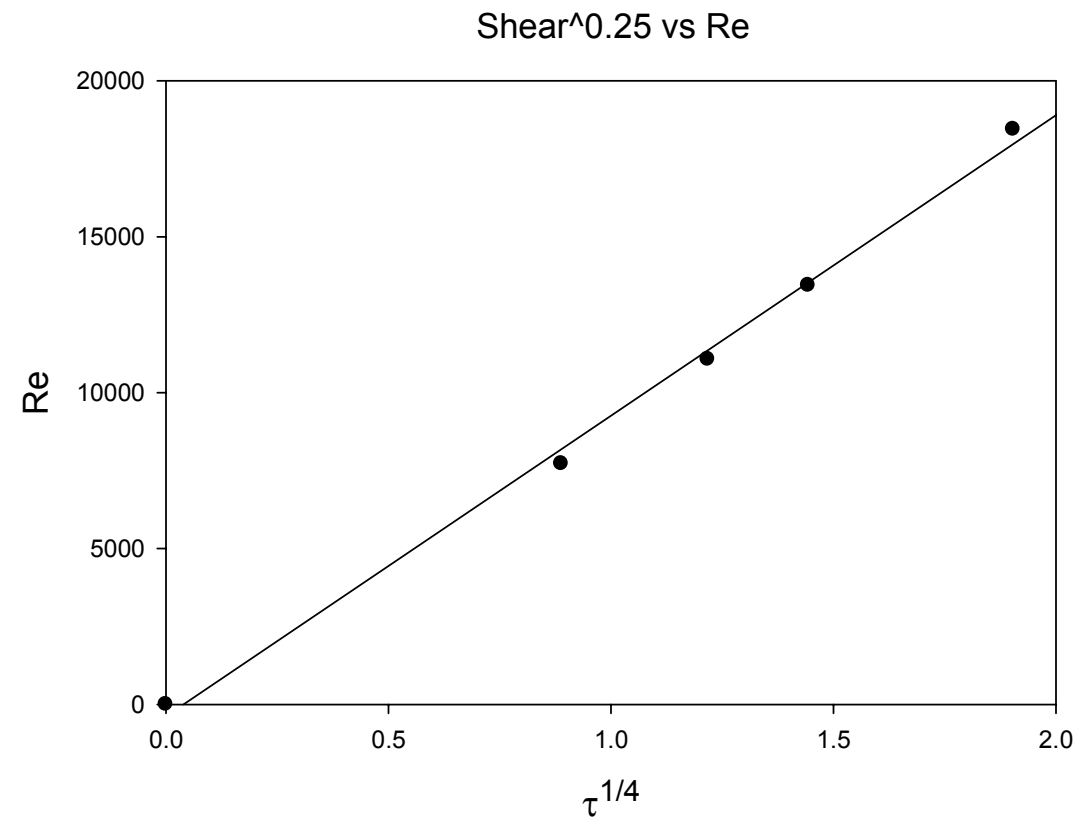


The linear regression equation was $\mathrm{y}=9635 \mathrm{x}-376.21, \mathrm{R}^{2}=0.9964$.

The $R e$ was then determined accordingly: 10126, 12583, and 16196. From equation below, the inlet inner diameter $d$ can be determined: $8.3,6.7$, and 5.2

$$
\operatorname{Re}=\frac{\rho d V}{\eta}=\frac{\rho d Q}{\eta A}=\frac{1.20 \times d \times 1 \times 10^{-3}}{1.81 \times 10^{-5} \times \frac{1}{4} \times \pi \times d^{2}}
$$

3) SET-A1 and SET-B1 use I-peripherally-located dosage table,

4) SET-C1 uses II-centrally-located dosage table. (Fig.1 below)

\begin{tabular}{|c|c|c|c|c|c|c|}
\hline SET & $\begin{array}{c}\text { External outlet } \\
\text { length (a) }\end{array}$ & $\begin{array}{c}\text { Inlet } \\
\text { length (b) }\end{array}$ & $\begin{array}{c}\text { Internal outlet } \\
\text { length (c) }\end{array}$ & $\begin{array}{c}\text { Powder dosage } \\
\text { length (d) }\end{array}$ & $\begin{array}{c}\text { Inlet outer } \\
\text { diameter (e) }\end{array}$ & $\begin{array}{c}\text { Inlet inner } \\
\text { diameter (f) }\end{array}$ \\
\hline A & 150 & 300 & 140 & 10 & 6.3 & 4.5 \\
\hline A1 & 144 & 300 & 134 & 10 & 7.0 & 5.2 \\
\hline B & 135 & 300 & 125 & 10 & 8.0 & 6.2 \\
\hline B1 & 133 & 300 & 123 & 10 & 8.5 & 6.7 \\
\hline C & 127 & 600 & 117 & 10 & 9.5 & 7.7 \\
\hline C1 & 121 & 600 & 111 & 10 & 10.1 & 8.3 \\
\hline D & 99 & 600 & 90 & 10 & 12.7 & 11.0 \\
\hline
\end{tabular}
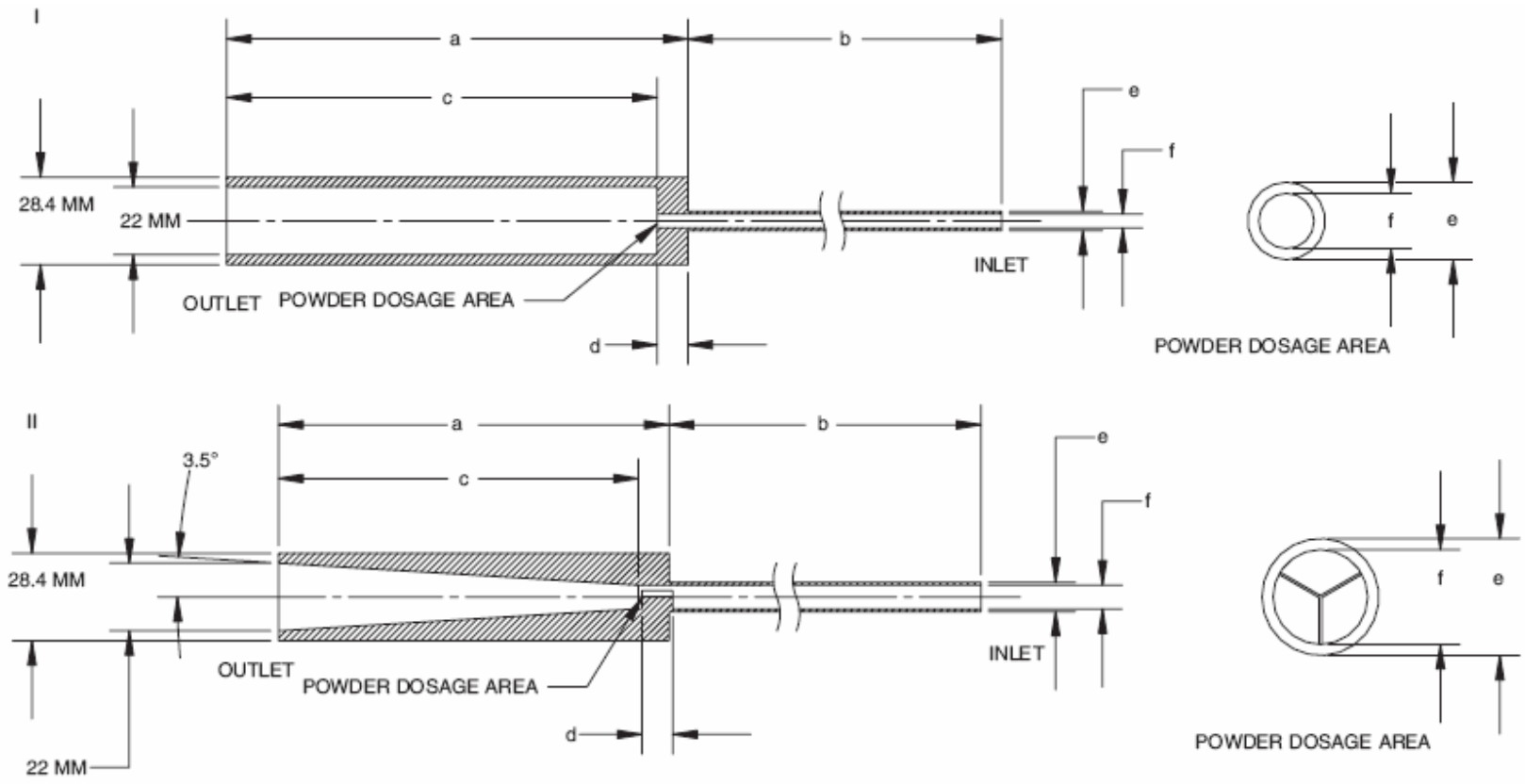

Fig. 1. Schematic diagram of standardized entrainment tubes (SETs) with I. peripherally-located dosage table and II. centrally-located dosage table. 
Appendix 5-B. TSLI Performance Data: Aerosolization Performance Using Three Extra SETs.

\begin{tabular}{|c|c|c|c|c|c|c|c|}
\hline Drug & Run & SET & $\begin{array}{c}\tau_{\mathrm{S}} \\
\left(\mathrm{N} / \mathrm{m}^{2}\right)\end{array}$ & $\begin{array}{c}\text { ED } \\
(\%)\end{array}$ & $\begin{array}{c}\mathrm{FPF} \\
(\%)\end{array}$ & $\begin{array}{c}\mathrm{FPF} \\
(\%)\end{array}$ & $\begin{array}{c}\tau_{\mathrm{s}} / \mathrm{FPFTD}{ }^{*} 10^{2} \\
\left(\mathrm{~N} / \mathrm{m}^{2} / \%^{*} 10^{2}\right)\end{array}$ \\
\hline DSCG & ML780 & A1 & 8.742 & 75.9 & 40.17 & 52.9 & 21.76 \\
\hline DSCG & ML780 & A1 & 8.742 & 75.4 & 43.88 & 58.2 & 19.92 \\
\hline DSCG & ML780 & A1 & 8.742 & 74.0 & 37.34 & 50.4 & 23.41 \\
\hline DSCG & ML780 & B1 & 2.775 & 65.5 & 31.33 & 47.8 & 8.86 \\
\hline DSCG & ML780 & B1 & 2.775 & 64.3 & 30.05 & 46.7 & 9.23 \\
\hline DSCG & ML780 & B1 & 2.775 & 62.7 & 30.69 & 48.9 & 9.04 \\
\hline DSCG & ML780 & C1 & 1.412 & 80.9 & 29.09 & 36.0 & 4.85 \\
\hline DSCG & ML780 & C1 & 1.412 & 78.4 & 26.42 & 33.7 & 5.34 \\
\hline DSCG & ML780 & C1 & 1.412 & 80.7 & 27.88 & 34.6 & 5.06 \\
\hline AS & Mann & A1 & 8.742 & 91.3 & 8.7 & 9.5 & 100.72 \\
\hline AS & Mann & A1 & 8.742 & 91.9 & 13.6 & 14.8 & 64.32 \\
\hline AS & Mann & A1 & 8.742 & 92.7 & 9.1 & 9.8 & 95.98 \\
\hline AS & Mann & B1 & 2.775 & 92.2 & 6.77 & 7.3 & 41.02 \\
\hline AS & Mann & B1 & 2.775 & 92.9 & 5.74 & 6.2 & 48.34 \\
\hline AS & Mann & B1 & 2.775 & 90.4 & 5.76 & 6.4 & 48.21 \\
\hline AS & Mann & C1 & 1.412 & 94.0 & 5.5 & 5.8 & 25.89 \\
\hline AS & Mann & C1 & 1.412 & 95.3 & 8.7 & 9.1 & 16.21 \\
\hline AS & Mann & C1 & 1.412 & 93.7 & 4.9 & 5.2 & 28.73 \\
\hline
\end{tabular}


Appendix 5-C. Schematic Calculation of Aerodynamic Shear Stress of DPI devices: e.g. Aerolizer ${ }^{\circledR}$

1) The mouthpiece length $\left(L=4.7 \times 10^{-3} \mathrm{~m}\right)$ of the Aerolizer ${ }^{\circledR}$ was determined using a caliper. The length was confirmed in the literature. (Pharm. Res., 2007, 24, 1450-1456).

2) The device pressure drop can be measured using a manometer. The airflow rate was set at $Q=60$ $\mathrm{L} / \mathrm{min}$. This parameter was also reported in literature $\left(\Delta P=1850 \mathrm{~N} / \mathrm{m}^{2}\right.$ at $\left.60 \mathrm{~L} / \mathrm{min}\right)$. (Pharm. Res.,2005, 22, 1445-1453).

3) The airflow velocity $\left(u_{\text {mean }}\right)$ that was volume-averaged throughout the whole device was $14.1 \mathrm{~m} / \mathrm{s}$ at $60 \mathrm{~L} / \mathrm{min}$. (Pharm. Res., 2005, 22, 1445-1453).

4) The critical diameter (d) of the device can be calculated according to Poiseuille's law: (Hickey, A.J., Ganderton, D., Fluid flow: in Pharmaceutical Process Engineering, 2001, Marcel Dekker, Inc., New York, NY, pp. 1-35).

$\Delta P=\frac{32 u_{\text {mean }} \mu L}{d^{2}} ; \quad$ is the air dynamic viscosity $1.81 \times 10^{-5} \mathrm{~kg} /(\mathrm{m} \mathrm{s}) ;$

The critical diameter, $d=1.44 \times 10^{-4} \mathrm{~m}$

5) The viscous shear stress $\left(\tau_{s}\right)$ was calculated according to Kolmogorov theory. (see Chapter 1, section 1.4.2).

$\tau_{s}=\mu\left(\frac{\varepsilon}{v}\right)^{1 / 2}$ where $\varepsilon \cong \frac{2\langle V\rangle^{3}}{d} \approx \frac{2(0.2 V)^{3}}{d}$ where $V=u_{\text {mean }}=14.1 \mathrm{~m} / \mathrm{s} ; v$ is the air kinematic viscosity $1.5 \times 10^{-6} \mathrm{~m}^{2} / \mathrm{s}$.

$\tau_{s}=8.248 \mathrm{~N} / \mathrm{m}^{2}$

It would be easy to calculate the other two aerodynamic parameters, Re and power:

$\operatorname{Re}=\frac{\rho_{a} D V}{\mu}=8881$, where $D$ was the diameter of the tube with grid, calculated from $Q$ and $V$.

power $=1.850 \mathrm{Nm} / \mathrm{s}$. 
Appendix 5-D. TSLI Performance Data: Aerosolization Performance Using Rotahaler® and Aerolizer®.

\begin{tabular}{|c|c|c|c|c|c|c|}
\hline Factor A & Factor B & Factor C & $\mathbf{E D ~}^{\mathbf{0}}$ & $\mathbf{F P F}_{\mathbf{T D}} \%$ & $\mathbf{F P F}_{\mathbf{E D}} \%$ & $\mathbf{F P M}$ \\
\hline ASMann & Gelatin & Rotahaler & 54.9 & 4.9 & 8.9 & 24.4 \\
\hline ASMann & Gelatin & Rotahaler & 77.7 & 10.2 & 13.2 & 51.2 \\
\hline ASMann & Gelatin & Rotahaler & 73.8 & 7.3 & 9.8 & 36.3 \\
\hline ASMann & HPMC & Rotahaler & 83.4 & 13.0 & 15.6 & 65.2 \\
\hline ASMann & HPMC & Rotahaler & 76.7 & 8.0 & 10.4 & 40.3 \\
\hline ASMann & HPMC & Rotahaler & 78.8 & 7.7 & 9.8 & 38.7 \\
\hline DSCGML780 & Gelatin & Rotahaler & 60.4 & 28.4 & 47.1 & 142.8 \\
\hline DSCGML780 & Gelatin & Rotahaler & 59.1 & 32.6 & 55.2 & 163.2 \\
\hline DSCGML780 & Gelatin & Rotahaler & 52.4 & 27.6 & 52.7 & 137.9 \\
\hline DSCGML780 & HPMC & Rotahaler & 55.1 & 25.6 & 46.4 & 127.3 \\
\hline DSCGML780 & HPMC & Rotahaler & 62.6 & 21.6 & 34.5 & 108.2 \\
\hline DSCGML780 & HPMC & Rotahaler & 60.4 & 33.5 & 55.5 & 166.3 \\
\hline ASMann & Gelatin & Aerolizer & 85.3 & 9.2 & 10.7 & 45.6 \\
\hline ASMann & Gelatin & Aerolizer & 86.4 & 10.9 & 12.6 & 54.8 \\
\hline ASMann & Gelatin & Aerolizer & 77.0 & 12.4 & 16.2 & 62.2 \\
\hline ASMann & HPMC & Aerolizer & 66.7 & 6.3 & 9.4 & 31.3 \\
\hline ASMann & HPMC & Aerolizer & 66.6 & 9.0 & 13.5 & 44.9 \\
\hline ASMann & HPMC & Aerolizer & 79.2 & 8.7 & 11.0 & 43.4 \\
\hline DSCGML780 & Gelatin & Aerolizer & 77.5 & 33.4 & 43.1 & 163.6 \\
\hline DSCGML780 & Gelatin & Aerolizer & 75.9 & 38.6 & 50.8 & 192.9 \\
\hline DSCGML780 & Gelatin & Aerolizer & 80.6 & 40.5 & 50.2 & 198.4 \\
\hline DSCGML780 & HPMC & Aerolizer & 82.6 & 37.7 & 45.6 & 186.1 \\
\hline DSCGML780 & HPMC & Aerolizer & 76.1 & 31.4 & 41.2 & 155.5 \\
\hline DSCGML780 & HPMC & Aerolizer & 82.2 & 36.6 & 44.6 & 179.6 \\
\hline
\end{tabular}


Appendix 5-E. Performance data of FP-Treh Before and After Storage in the Desiccator.

\begin{tabular}{|c|c|c|c|c|c|}
\hline Drug & Lactose & Tube & ED & FPF $_{\text {TD }}$ & FPF $_{\text {ED }}$ \\
\hline \multicolumn{7}{|c|}{ Before Storage* } \\
\hline FP & Treh & A & 64.9 & 7.9 & 12.1 \\
\hline FP & Treh & A & 67.3 & 8.1 & 12.1 \\
\hline FP & Treh & A & 65.7 & 11.0 & 16.7 \\
\hline FP & Treh & B & 66.6 & 4.9 & 7.3 \\
\hline FP & Treh & B & 64.7 & 5.4 & 8.3 \\
\hline FP & Treh & B & 65.8 & 5.2 & 7.9 \\
\hline FP & Treh & C & 87.6 & 8.3 & 9.4 \\
\hline FP & Treh & C & 86.2 & 11.4 & 13.2 \\
\hline FP & Treh & C & 86.9 & 8.8 & 10.2 \\
\hline FP & Treh & D & 91.7 & 7.1 & 7.7 \\
\hline FP & Treh & D & 88.4 & 6.5 & 7.4 \\
\hline FP & Treh & D & 90.6 & 6.7 & 7.4 \\
\hline \multicolumn{7}{|c|}{ After Storage** } \\
\hline FP & Treh & A & 66.6 & 6.3 & 9.5 \\
\hline FP & Treh & A & 63.0 & 6.9 & 11.0 \\
\hline FP & Treh & A & 58.3 & 5.1 & 8.8 \\
\hline FP & Treh & A1 & 67.6 & 6.7 & 10.0 \\
\hline FP & Treh & A1 & 58.9 & 6.0 & 10.1 \\
\hline FP & Treh & A1 & 60.5 & 3.9 & 6.5 \\
\hline FP & Treh & B & 67.3 & 5.0 & 7.4 \\
\hline FP & Treh & B & 67.6 & 6.7 & 10.0 \\
\hline FP & Treh & B & 60.1 & 4.2 & 7.0 \\
\hline FP & Treh & B1 & 63.9 & 3.9 & 6.2 \\
\hline FP & Treh & B1 & 66.2 & 4.0 & 6.0 \\
\hline FP & Treh & B1 & 70.0 & 4.8 & 6.9 \\
\hline FP & Treh & C & 85.1 & 4.8 & 5.6 \\
\hline FP & Treh & C & 89.0 & 5.2 & 5.8 \\
\hline FP & Treh & C & 84.4 & 4.4 & 5.3 \\
\hline FP & Treh & C1 & 79.7 & 4.9 & 6.2 \\
\hline FP & Treh & C1 & 79.9 & 3.0 & 3.8 \\
\hline FP & Treh & C1 & 75.3 & 3.1 & 4.1 \\
\hline FP & Treh & D & 87.9 & 2.5 & 2.9 \\
\hline FP & Treh & D & 85.3 & 1.9 & 2.2 \\
\hline FP & Treh & D & 87.9 & 5.3 & 6.0 \\
\hline \multicolumn{7}{|l|}{} \\
\hline
\end{tabular}

* Data adapted from Chapter 4

** Newly generated data 
Appendix 6-A. The Amount of Fatty Acid Associated on the Surface of Lactose Monohydrate Powder Treated with Stearic Acid (SA), Palmitic Acid (PA), Myristic Acid (MA) and Lauric Acid (LA).

\begin{tabular}{|c|c|c|c|c|c|c|c|c|}
\hline $\begin{array}{c}\text { Equil. } \\
\text { Conc. }\end{array}$ & $\begin{array}{c}\text { SA/SV } \\
(\mu \mathbf{g} / \mathbf{g})\end{array}$ & $\begin{array}{c}\text { SA/ML } \\
(\mu \mathbf{g} / \mathbf{g})\end{array}$ & $\begin{array}{c}\text { PA/SV } \\
(\mu \mathbf{g} / \mathbf{g})\end{array}$ & $\begin{array}{c}\text { PA/ML } \\
(\mu \mathbf{g} / \mathbf{g})\end{array}$ & $\begin{array}{c}\text { MA/SV } \\
(\mu \mathbf{g} / \mathbf{g})\end{array}$ & $\begin{array}{c}\text { MA/ML } \\
(\mu \mathbf{g} / \mathbf{g})\end{array}$ & $\begin{array}{c}\text { LA/SV } \\
(\mu \mathbf{g} / \mathbf{g})\end{array}$ & $\begin{array}{c}\text { LA/ML } \\
(\mu \mathbf{g} / \mathbf{g})\end{array}$ \\
\hline 0.2 & -- & 4.14 & 6.22 & 9.92 & 12.92 & 14.05 & 6.08 & - \\
\hline 0.4 & 7.16 & 12.80 & 7.29 & 12.19 & 17.76 & 19.03 & 8.17 & 15.92 \\
\hline 0.6 & 7.59 & 13.17 & 12.00 & 14.73 & 23.03 & 21.91 & 20.14 & 22.67 \\
\hline 0.8 & 9.89 & 13.17 & 17.58 & 23.55 & 23.92 & 30.19 & 41.66 & 44.48 \\
\hline 1.6 & 33.37 & 41.88 & 28.33 & 47.94 & 39.18 & 26.94 & 57.22 & 71.78 \\
\hline 2.4 & 63.71 & 67.92 & 41.42 & 73.32 & 61.39 & 67.01 & 74.17 & 102.39 \\
\hline 3.2 & 91.87 & 91.63 & 77.47 & 84.47 & 70.19 & 99.46 & 104.26 & 147.34 \\
\hline 4.8 & 143.29 & 131.98 & 116.97 & 126.05 & 114.52 & 126.76 & 167.01 & 216.16 \\
\hline 6.0 & 166.40 & 194.05 & 143.30 & 159.60 & 107.37 & 178.87 & 183.85 & 246.32 \\
\hline 8.0 & 242.40 & 197.56 & 183.65 & 236.14 & 185.86 & 226.02 & 217.56 & - \\
\hline
\end{tabular}


Appendix 6-B. The Aerosolization Performance after Stearic Acid Coating (AS formulation)

\begin{tabular}{|c|c|c|c|c|c|c|}
\hline Drug & Carrier & Tube & Conc (\%) & ED (\%) & $\mathrm{FPF}_{\mathrm{TD}}(\%)$ & FPF $_{\text {ED }}(\%)$ \\
\hline AS & SV473-control & A & 2.0 & 76.75 & 12.4 & 16.1 \\
\hline AS & SV473-control & A & 2.0 & 75.36 & 11.9 & 15.8 \\
\hline AS & SV473-control & A & 2.0 & 80.25 & 12.0 & 15.0 \\
\hline AS & SV473-control & $B$ & 2.0 & 84 & 8.7 & 10.4 \\
\hline AS & SV473-control & B & 2.0 & 81.4 & 8.8 & 10.8 \\
\hline AS & SV473-control & $B$ & 2.0 & 77.24 & 7.5 & 9.7 \\
\hline AS & SV473-control & $\mathrm{C}$ & 2.0 & 84.63 & 7.8 & 9.3 \\
\hline AS & SV473-control & C & 2.0 & 84.77 & 8.1 & 9.5 \\
\hline AS & SV473-control & $\mathrm{C}$ & 2.0 & 86.89 & 8.6 & 9.9 \\
\hline AS & SV473-control & $\mathrm{D}$ & 2.0 & 82.45 & 3.8 & 4.6 \\
\hline AS & SV473-control & $\mathrm{D}$ & 2.0 & 86.32 & 6.1 & 7.1 \\
\hline AS & SV473-control & $\mathrm{D}$ & 2.0 & 90.8 & 7.2 & 8.0 \\
\hline AS & SV473-lowSA & A & 2.0 & 90.1 & 14.6 & 16.2 \\
\hline AS & SV473-lowSA & A & 2.0 & 91.9 & 17.0 & 18.5 \\
\hline AS & SV473-lowSA & A & 2.0 & 87.1 & 13.2 & 15.2 \\
\hline AS & SV473-lowSA & $B$ & 2.0 & 88.2 & 11.4 & 12.9 \\
\hline AS & SV473-lowSA & $\mathrm{B}$ & 2.0 & 84.9 & 10.9 & 12.9 \\
\hline AS & SV473-lowSA & B & 2.0 & 85.9 & 9.1 & 10.6 \\
\hline AS & SV473-lowSA & C & 2.0 & 90.4 & 9.1 & 10.1 \\
\hline AS & SV473-lowSA & C & 2.0 & 88.0 & 10.5 & 12.0 \\
\hline AS & SV473-lowSA & $\mathrm{C}$ & 2.0 & 91.1 & 10.0 & 11.0 \\
\hline AS & SV473-lowSA & $\mathrm{D}$ & 2.0 & 91.4 & 7.2 & 7.9 \\
\hline AS & SV473-lowSA & $\mathrm{D}$ & 2.0 & 84.6 & 5.1 & 6.1 \\
\hline AS & SV473-lowSA & $\mathrm{D}$ & 2.0 & 92.3 & 5.6 & 6.1 \\
\hline AS & SV473-highSA & A & 2.0 & 83.1 & 20.5 & 24.7 \\
\hline AS & SV473-highSA & A & 2.0 & 82.4 & 18.5 & 22.5 \\
\hline AS & SV473-highSA & A & 2.0 & 79.5 & 16.7 & 21.0 \\
\hline AS & SV473-highSA & B & 2.0 & 85.3 & 9.1 & 10.7 \\
\hline AS & SV473-highSA & $B$ & 2.0 & 82.5 & 10.8 & 13.1 \\
\hline AS & SV473-highSA & $B$ & 2.0 & 86.7 & 15.8 & 18.2 \\
\hline AS & SV473-highSA & C & 2.0 & 83.0 & 8.1 & 9.8 \\
\hline AS & SV473-highSA & C & 2.0 & 92.2 & 10.5 & 11.4 \\
\hline AS & SV473-highSA & C & 2.0 & 83.9 & 8.5 & 10.1 \\
\hline AS & SV473-highSA & $\bar{D}$ & 2.0 & 89.5 & 9.7 & 10.9 \\
\hline AS & SV473-highSA & D & 2.0 & 90.4 & 7.8 & 8.6 \\
\hline AS & SV473-highSA & $\mathrm{D}$ & 2.0 & 91.0 & 7.9 & 8.6 \\
\hline
\end{tabular}


Appendix 6-B' (continued).

\begin{tabular}{|c|c|c|c|c|c|c|}
\hline Drug & Carrier & Tube & Conc (\%) & ED (\%) & FPF $_{\text {TD }}(\%)$ & FPF $_{\text {ED }}(\%)$ \\
\hline AS & ML058-control & A & 2.0 & 78.4 & 35.3 & 45.0 \\
\hline AS & ML058-control & A & 2.0 & 77.1 & 33.4 & 43.3 \\
\hline AS & ML058-control & A & 2.0 & 76.1 & 33.2 & 43.6 \\
\hline AS & ML058-control & B & 2.0 & 76.6 & 31.3 & 40.9 \\
\hline AS & ML058-control & B & 2.0 & 76.3 & 31.6 & 41.4 \\
\hline AS & ML058-control & B & 2.0 & 74.6 & 28.7 & 38.4 \\
\hline AS & ML058-control & C & 2.0 & 78.3 & 24.8 & 31.7 \\
\hline AS & ML058-control & C & 2.0 & 77.8 & 24.3 & 31.2 \\
\hline AS & ML058-control & C & 2.0 & 79 & 23.7 & 29.9 \\
\hline AS & ML058-control & D & 2.0 & 83.4 & 20.0 & 23.9 \\
\hline AS & ML058-control & D & 2.0 & 83.1 & 18.5 & 22.3 \\
\hline AS & ML058-control & D & 2.0 & 84.1 & 19.6 & 23.3 \\
\hline AS & ML058-lowSA & A & 2.0 & 80.5 & 35.9 & 44.6 \\
\hline AS & ML058-lowSA & A & 2.0 & 80.0 & 39.3 & 49.1 \\
\hline AS & ML058-lowSA & A & 2.0 & 84.1 & 42.5 & 50.6 \\
\hline AS & ML058-lowSA & D & 2.0 & 84.5 & 21.6 & 25.5 \\
\hline AS & ML058-lowSA & D & 2.0 & 80.8 & 20.7 & 25.7 \\
\hline AS & ML058-lowSA & D & 2.0 & 82.1 & 19.2 & 23.4 \\
\hline AS & ML058-highSA & A & 2.0 & 81.5 & 42.2 & 51.7 \\
\hline AS & ML058-highSA & A & 2.0 & 79.9 & 39.2 & 49.0 \\
\hline AS & ML058-highSA & A & 2.0 & 80.2 & 40.6 & 50.7 \\
\hline AS & ML058-highSA & B & 2.0 & 74.2 & 33.7 & 45.3 \\
\hline AS & ML058-highSA & B & 2.0 & 77.3 & 34.2 & 44.3 \\
\hline AS & ML058-highSA & B & 2.0 & 77.4 & 37.7 & 48.7 \\
\hline AS & ML058-highSA & C & 2.0 & 86.8 & 31.1 & 35.8 \\
\hline AS & ML058-highSA & C & 2.0 & 85.4 & 33.0 & 38.7 \\
\hline AS & ML058-highSA & C & 2.0 & 87.5 & 33.4 & 38.1 \\
\hline AS & ML058-highSA & D & 2.0 & 88.0 & 20.7 & 23.6 \\
\hline AS & ML058-highSA & D & 2.0 & 87.5 & 22.9 & 26.2 \\
\hline AS & ML058-highSA & D & 2.0 & 86.1 & 23.0 & 26.7 \\
\hline & & & & & & \\
\hline
\end{tabular}


Appendix 6-C. The Aerosolization Performance after Stearic Acid Coating (FP formulation)

\begin{tabular}{|c|c|c|c|c|c|c|}
\hline Drug & Carrier & Tube & Conc (\%) & ED (\%) & FPF $_{\mathrm{TD}}(\%)$ & FPF $_{E D}(\%)$ \\
\hline FP & SV473-control & A & 2.0 & 49.5 & 4.7 & 9.6 \\
\hline FP & SV473-control & $A$ & 2.0 & 55.5 & 3.6 & 6.5 \\
\hline FP & SV473-control & $A$ & 2.0 & 54.8 & 5.1 & 9.2 \\
\hline FP & SV473-control & $\mathrm{B}$ & 2.0 & 51.0 & 4.1 & 8.1 \\
\hline FP & SV473-control & B & 2.0 & 53.4 & 4.2 & 7.8 \\
\hline FP & SV473-control & $B$ & 2.0 & 50.6 & 3.4 & 6.7 \\
\hline FP & SV473-control & $\mathrm{C}$ & 2.0 & 83.3 & 1.9 & 2.3 \\
\hline FP & SV473-control & C & 2.0 & 82.5 & 2.2 & 2.7 \\
\hline FP & SV473-control & $\mathrm{C}$ & 2.0 & 81.7 & 3.9 & 4.7 \\
\hline FP & SV473-control & $\mathrm{D}$ & 2.0 & 75.4 & 1.4 & 1.8 \\
\hline FP & SV473-control & $\mathrm{D}$ & 2.0 & 74.3 & 1.5 & 2.0 \\
\hline FP & SV473-control & $\mathrm{D}$ & 2.0 & 77.2 & 1.8 & 2.3 \\
\hline FP & ML058-control & A & 2.0 & 54.5 & 15.0 & 27.5 \\
\hline FP & ML058-control & A & 2.0 & 51.8 & 13.6 & 26.3 \\
\hline FP & ML058-control & A & 2.0 & 54.1 & 13.8 & 25.4 \\
\hline FP & ML058-control & $\mathrm{B}$ & 2.0 & 44.2 & 9.6 & 21.6 \\
\hline FP & ML058-control & $\mathrm{B}$ & 2.0 & 45.9 & 9.5 & 20.8 \\
\hline FP & ML058-control & $\mathrm{B}$ & 2.0 & 50.7 & 12.1 & 23.9 \\
\hline FP & ML058-control & $\mathrm{C}$ & 2.0 & 80.1 & 11.9 & 14.8 \\
\hline FP & ML058-control & $\mathrm{C}$ & 2.0 & 80.4 & 11.2 & 14.0 \\
\hline FP & ML058-control & $\mathrm{C}$ & 2.0 & 78.2 & 9.7 & 12.4 \\
\hline FP & ML058-control & $\mathrm{D}$ & 2.0 & 81.4 & 10.3 & 12.6 \\
\hline FP & ML058-control & $\mathrm{D}$ & 2.0 & 80.0 & 9.7 & 12.1 \\
\hline FP & ML058-control & $\mathrm{D}$ & 2.0 & 81.3 & 8.8 & 10.8 \\
\hline FP & SV473-lowSA & A & 2.0 & 63.4 & 11.8 & 18.7 \\
\hline FP & SV473-lowSA & A & 2.0 & 63.1 & 11.0 & 17.5 \\
\hline FP & SV473-lowSA & $\mathrm{D}$ & 2.0 & 81.6 & 6.1 & 7.5 \\
\hline FP & SV473-lowSA & $\mathrm{D}$ & 2.0 & 81.4 & 5.7 & 7.0 \\
\hline FP & SV473-highSA & A & 2.0 & 66.9 & 14.9 & 22.3 \\
\hline FP & SV473-highSA & A & 2.0 & 68.5 & 17.6 & 25.7 \\
\hline FP & SV473-highSA & $\mathrm{D}$ & 2.0 & 84.3 & 7.0 & 8.3 \\
\hline FP & SV473-highSA & $\mathrm{D}$ & 2.0 & 83.2 & 6.7 & 8.1 \\
\hline FP & ML058-IowSA & A & 2.0 & 58.8 & 21.9 & 37.2 \\
\hline FP & ML058-lowSA & A & 2.0 & 59.1 & 22.0 & 37.3 \\
\hline FP & ML058-lowSA & $\mathrm{D}$ & 2.0 & 82.8 & 20.5 & 24.7 \\
\hline FP & ML058-lowSA & $\mathrm{D}$ & 2.0 & 83.5 & 20.8 & 24.9 \\
\hline FP & ML058-highSA & $A$ & 2.0 & 67.3 & 26.6 & 39.5 \\
\hline FP & ML058-highSA & A & 2.0 & 67.2 & 24.5 & 36.5 \\
\hline FP & ML058-highSA & $\mathrm{D}$ & 2.0 & 81.6 & 16.6 & 20.3 \\
\hline FP & ML058-highSA & $\mathrm{D}$ & 2.0 & 84.0 & 22.1 & 26.3 \\
\hline
\end{tabular}

